 \\ Michigan Technological University Create the Future Digital Commons @ Michigan Tech
}

Dissertations, Master's Theses and Master's Reports - Open

Dissertations, Master's Theses and Master's

Reports

2012

\section{Structural response and dynamics of fluid-structure-control interaction in wind turbine blades}

Lucan Ignacio Lago

Michigan Technological University

Follow this and additional works at: https://digitalcommons.mtu.edu/etds

Part of the Mechanical Engineering Commons

Copyright 2012 Lucan Ignacio Lago

\section{Recommended Citation}

Lago, Lucan Ignacio, "Structural response and dynamics of fluid-structure-control interaction in wind turbine blades", Dissertation, Michigan Technological University, 2012.

https://doi.org/10.37099/mtu.dc.etds/380

Follow this and additional works at: https://digitalcommons.mtu.edu/etds

Part of the Mechanical Engineering Commons 
STRUCTURAL RESPONSE AND DYNAMICS OF

FLUID-STRUCTURE-CONTROL INTERACTION IN WIND TURBINE BLADES

By

Lucas Ignacio Lago

\begin{abstract}
A DISSERTATION
Submitted in partial fulfillment of the requirements for the degree of DOCTOR OF PHILOSOPHY

(Mechanical Engineering - Engineering Mechanics)
\end{abstract}

MICHIGAN TECHNOLOGICAL UNIVERSITY

2012

(c) 2012 Lucas Ignacio Lago 
This dissertation, "Structural Response and Dynamics of Fluid-Structure-Control Interaction in Wind Turbine Blades," is hereby approved in partial fulfillment for the requirements for the Degree of DOCTOR OF PHILOSOPHY in MECHANICAL ENGINEERING ENGINEERING MECHANICS.

Department of Mechanical Engineering - Engineering Mechanics

Advisor:

Dr. Fernando L. Ponta

Department Chair:

Dr. William W. Predebon

Date: 
To my father, Enrique Lago \& to my son Joaquin. 


\section{Contents}

List of Figures $\ldots \ldots \ldots \ldots \ldots \ldots$

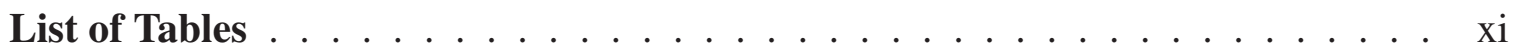

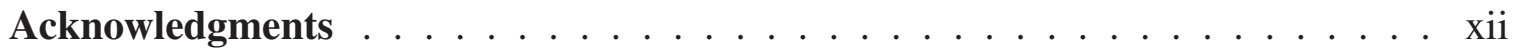

List of Symbols . . . . . . . . . . . . . . . . . . . xiv

Abstract ......................... . . . . . . .

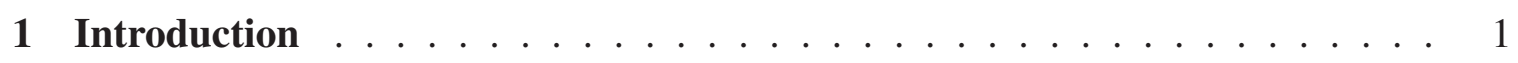



1.2 Dissertation Outline . . . . . . . . . . . . . . 7

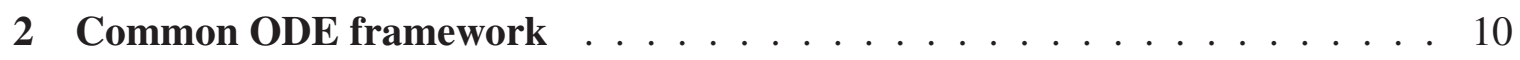

2.1 Numerical implementation . . . . . . . . . . . . . . . . 11

2.2 The Level-1 flow model . . . . . . . . . . . . . . . . . . . . 14

2.3 The stiff multistep BDF-ODE solver . . . . . . . . . . . . . 17

3 The level-1 flow model . . . . . . . . . . . . . . . . . . . . . . . 18 
3.1 Theoretical Background: The Classic Blade Element Momentum theory . . 18

3.1.1 Corrective factors to BEM theory . . . . . . . . . 25

3.1.2 Limitations of the classic BEM theory for large deformations . . . . 26

3.2 Large Sectional Rotation BEM (LSR-BEM) . . . . . . . . . . . . 26

3.2.1 Iterative solution of the induction factors $\ldots \ldots . \ldots 33$

3.2.2 Interference model for the "turbulent-wake" state . . . . . . . . . 33

3.3 The Flow Model Interface $(\mathrm{FMI}) \ldots \ldots$. . . . . . . . . . . 36

3.3.1 Pre-processing of the airfoil aerodynamic coefficients . . . . . . . 36

3.3.2 Orthogonal projection matrices associated with the action of nacelle mechanisms . . . . . . . . . . . . . . . . . 43

3.3.3 Coupling with the structural model . . . . . . . . . . . 45

3.3 .4 Wind input data . . . . . . . . . . . . . 46

4 The Structural Model . . . . . . . . . . . . . . . . . . . . . . . . . . . 49

4.1 The Generalized Timoshenko Beam Model (GTBM) . . . . . . . . . . . . 50

4.2 The $1 \mathrm{D}$ model . . . . . . . . . . . . . . . . . . . 54

4.3 Constitutive relations of composite materials . . . . . . . . . . . 57

5 Numerical Experimentation . . . . . . . . . . . . . . . . . 65

5.1 Basic blade modelling and aerodynamic properties . . . . . . . . . 66

5.1.1 Blade constructive aspects . . . . . . . . . . . 70

5.1 .2 Blade structural properties . . . . . . . . . . . 72

5.1.3 Turbine specifications . . . . . . . . . . . . 77

5.2 Validation Tests . . . . . . . . . . . . . . . . 84

5.2.1 Aeroelastic steady-state case . . . . . . . . . . . . . . . . 84 
5.2.2 Vibrational modes around the aeroelastic steady-state . . . . . . . . 89

5.2.3 Recovering of displacements, strains and stresses for the blade sections . . . . . . . . . . . . . . . . . . 90

5.2.4 Dynamic simulation of rotor start-up . . . . . . . . . . . . 90

5.2.5 Blade pitch control for power limitation at wind speeds above the rated. . . . . . . . . . . . . . . 95

5.3 Blade Adaptiveness . . . . . . . . . . . . . . . . . . 101

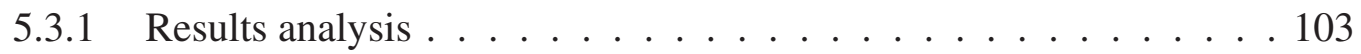

5.4 Gravitational forces . . . . . . . . . . . . . . . . . . 111

5.4.1 Gravitational effects during rotor acceleration . . . . . . . . . 112

5.4.2 Rumbling effects induced by gravitational forces . . . . . . . . . . 114

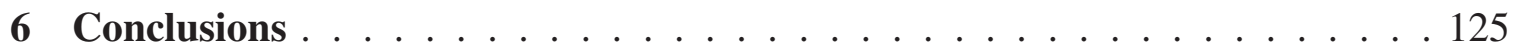

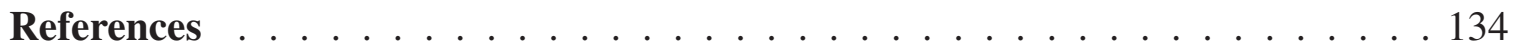

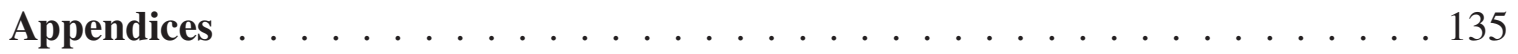

A Recovery of three dimensional variables . . . . . . . . . . . . 136

A.1 Computation of the sections' warping . . . . . . . . . . . 136

A.2 Computation of stresses and deformations . . . . . . . . . . . 137

A.3 Displacements of the blade sections . . . . . . . . . . . . . 138

A.4 Other applications of the dimensional reduction model . . . . . . . . . . 139

B Copyright agreements . . . . . . . . . . . . . . . . 143

B.1 Copyright statement for Chapter $1 \ldots \ldots$. . . . . . . . . . 144

B.2 Copyright statement for Chapter $3 \ldots \ldots$. . . . . . . . . . . 145 


\section{LIST OF FIGURES}

1.1 State-of-the art multi-megawatt wind turbines. . . . . . . . . . . . . 2

1.2 REPower $5 \mathrm{M}$ wind turbine blades. . . . . . . . . . . . . . 3

1.3 Proportional cost of wind-turbine subsystems. . . . . . . . . . . . . 4

1.4 Wind-turbine blades convoy on the way to Scout Moor wind farm, England. 5

2.1 Pseudo-code diagram. . . . . . . . . . . . . . . . . . 11

2.2 Common ODE framework diagram. . . . . . . . . . . . . . 13

2.3 Pseudo-code diagram for the common framework on the Level-1 flow model option. . . . . . . . . . . . . . . . . . . . . 16

3.1 Aerodynamic efforts on an airfoil. . . . . . . . . . . . . . . . 19

3.2 Helical vortex wake shed by rotor with three blades at uniform circulation. . 20

3.3 Top view analysis of an HAWT blade cross-section. . . . . . . . . . . . . . 21

3.4 A blade element sweeping out an annular ring. . . . . . . . . . . . . 22

3.5 Hub coordinate system. . . . . . . . . . . . . . . . . . 28

3.6 Cone and tilt angles definition. . . . . . . . . . . . . . . . . 29

3.7 Blade coordinate system. . . . . . . . . . . . . . . . 30

3.8 Empirical relations fitting the Lock experimental data. . . . . . . . . . . . . 34 
3.9 Power law relation fitting Lock experimental data. . . . . . . . . . . . . 36

3.10 Viterna's extrapolation of airfoil aerodynamic coefficients . . . . . . . . . . 40

3.11 Hysteresis loops obtained from measured $C_{M}$ coefficients on a NACA 4415 airfoil for a determined sinusoidal oscillation . . . . . . . . . . . . 42

$3.12 C_{N}$ coefficients hysteresis loops computed for a NACA 4415 airfoil at unusual angles of attack using the Beddoes-Leishman model. . . . . . . . . . 42

3.13 Moment coefficient $C_{M}$ benchmark for an S809 airfoil. Wind tunnel data vs. computations with our actual implementation of the dynamic stall model ............................. 44

3.14 Moment coefficient $C_{M}$ benchmark for an S809 airfoil. Wind tunnel data vs. computations with our actual implementation of the dynamic stall



4.1 Example of blade-section structural architecture representative of current commercial blade designs. . . . . . . . . . . . . . . . . . . 49

4.2 Generalized Timoshenko theory: Schematic of the reference line, orthogonal triads, and beam sections before and after deformation. . . . . . . . . 51

4.3 Reference frames involved in a laminate and their orientations. . . . . . . . 64

5.1 Chord distribution along the blade. . . . . . . . . . . 66

5.2 Profiles of ellipsoidal sections . . . . . . . . . . . . 68

5.3 Profiles of airfoil master sections . . . . . . . . . . . . . . . 68

5.4 Aerodynamic coefficients for standard airfoils . . . . . . . . . . . . 69

5.5 Profiles of morphed sections . . . . . . . . . . . . 73

5.6 Finite element meshes for morphed sections. . . . . . . . . . . . . . 79

5.7 Finite element meshes for the master sections (a) . . . . . . . . . . . . 80

5.8 Finite element meshes for the master sections (b). . . . . . . . . . . 81

5.9 Finite element meshes for the master sections (c). . . . . . . . . . . . 82 
5.10 Top view of the internal blade layout. . . . . . . . . . . . . 83

5.11 Aeroelastic steady state. Linear displacements of the reference-line. . . . . 85

5.12 Aeroelastic steady state. Angular displacements of the reference-line. . . . 86

5.13 Timoshenko measures of deformation (a). . . . . . . . . . . . . . . 87

5.14 Timoshenko measures of deformation (b) . . . . . . . . . . . . . . 88

5.15 Aerolastic steady-state vibrational modes \# 1,2 and 3. . . . . . . . . . 91

5.16 Aerolastic steady-state vibrational modes \# 4, 7 and 10. . . . . . . . . . 92

$5.17 Z_{11}, Z_{12}$ and $Z_{13}$ components of the Jaumann-Biot-Cauchy stress tensor . . 93

$5.18 Z_{22}, Z_{23}$ and $Z_{33}$ components of the Jaumann-Biot-Cauchy stress tensor . . 94

5.19 Start-up simulation, acceleration ramp. . . . . . . . . . . . 95

5.20 Linear displacements evolution for a point located at the $95 \%$ of the blade's reference-line during start-up. . . . . . . . . . . . . . 96

5.21 Angular displacements evolution for a point located at the $95 \%$ of the blade's reference-line during start-up. . . . . . . . . . . . . . 97

5.22 Aerodynamic forces evolution for a point located at the $95 \%$ of the blade's reference-line during start-up . . . . . . . . . . . . . . . 98

5.23 Rotor torque evolution during start-up. . . . . . . . . . . . . . . . . 99

5.24 Power output evolution during start-up. . . . . . . . . . . . . . . . . 99

5.25 Comparison of the reference-line position along the blade span. . . . . . . . 102

5.26 Blade configuration comparison. . . . . . . . . . . . . 103

5.27 Tip speed ratio evolution during blade adaptiveness simulations. . . . . . . 103

5.28 Blade adaptiveness, linear displacements comparisons. . . . . . . . . . 106

5.29 Blade adaptiveness, rotor thrust force comparisons. . . . . . . . . . . 107

5.30 Blade adaptiveness, rotor torque comparisons. . . . . . . . . . . . . 108 
5.31 Blade adaptiveness, power output comparisons. . . . . . . . . . . . . 109

5.32 Blade adaptiveness, blade section torsion angle comparisons. . . . . . . . . 110

5.33 Time evolution of the aerodynamic chord-normal force on a blade under acceleration from $\lambda=1$ to $\lambda=4$ with gravitational forces included. . . . . 113

5.34 Block diagram of the dynamic response of the aeroelastic system. . . . . . 115

5.35 Gravitational forces. . . . . . . . . . . . . . . 118

5.36 Chord-wise component of the blade displacement for a blade section located at the $95 \%$ of the span. . . . . . . . . . . . . . . . . 119

5.37 Chord-normal component of the blade displacement for a blade section located at the $95 \%$ of the span. . . . . . . . . . . . . . 120

5.38 Angle of torsion for a blade section located at the $95 \%$ of the span. . . . . . 121

5.39 Angle of the flow incident on the blade section located at the $95 \%$ of the span. . . . . . . . . . . . . . . . . . . 122

5.40 Angle of attack on the blade section located at the $95 \%$ of the span. . . . . 123

5.41 Chord-normal component of the aerodynamic force on the blade section located at the $95 \%$ of the span. . . . . . . . . . . . . . . 124 


\section{LIST OF TABLES}

1.1 Main characteristics of the Repower 5MW wind turbine. . . . . . . . . . 2

3.1 Mean Square Error (MSE) of the different empirical approximations to Lock's experimental data. . . . . . . . . . . . . . . . . . . 35

5.1 Distributed blade aerodynamic properties. . . . . . . . . . . . . . 67

5.2 Summary of structural details. . . . . . . . . . . . . 71

5.3 Summary of Baseline Blade Material Properties . . . . . . . . . . . . . 72

5.4 Blade section structural centering and aerodynamic coefficient reference point ............................. 74

5.5 Distributed blade structural properties . . . . . . . . . . . . 76

5.6 Global turbine parameters . . . . . . . . . . . . 78

5.7 List of frequencies and dominant components for ten modes of vibration of the aerolastic steady-state solution . . . . . . . . . . . . . . . 89

5.8 Frequencies comparison for the first three modes . . . . . . . . . . . . 89

5.9 Sensitivity of Aerodynamic Power to Blade Pitch. . . . . . . . . . . . . 100 


\section{ACKNOWLEDGMENTS}

The time has come. An important stage in my life comes to an end, and with it comes the time to look back to be grateful for the ones that shared with me this path in one way or another.

I want to thank my advisor, Dr. Fernando Ponta, for believing in me and for his guidance and support through the years. He has been inspiring my research curiosity since my early undergraduate days back in Buenos Aires. I will always keep the great memories of the rich discussions we had, not only the ones that made me grow at a technical and scientific level but most important, the ones that made me grow as a better human being.

Thanks so much to my family for making me feel all their love from such a long physical distance. Thanks to my wife Marisa, for the unconditional support and the love in every little thing every single day, and thanks to my son Joaquin, the greatest gift life could have given me.

I would like to thank the whole research group at MTU, Liu Chen, Anurag Rajan and Pankaj Jagadale for all the help and all the time we spent together sharing and learning new things. Also, I would like to thank specially Dr. Alejandro Otero for his invaluable help, discussions and dedication to all my questions during my $\mathrm{PhD}$ career.

Thanks to the Michigan Technological University for the financial support during my first years, and to the U. S. National Science Foundation for the support though the grants CEBET-0933058 and CEBET-0952218.

During my time at MTU I had the pleasure to meet an incredible and diverse group of people, with them we shared unforgettable times through these years. Thanks for everything John and Julia Munson, Oren Tikkanen, Greg Wright, Ezequiel Medici, Julio Rivera, and all the NOSOTROS Student Organization. And to my friends back in Argentina, thanks to honor the word friendship as you do. 


\section{List OF SYMBOLS}

\section{Symbols in Chapter 3}






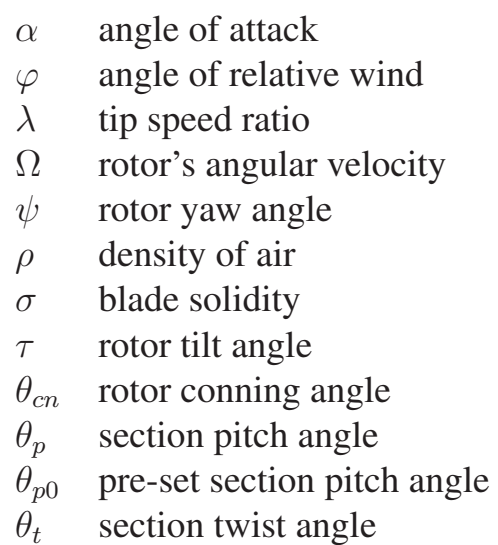

\section{Symbols in Chapter 4}






\title{
Structural Response and Dynamics of Fluid-Structure-Control Interaction in Wind Turbine Blades
}

\author{
Lucas Ignacio Lago \\ Michigan Technological University, 2012 \\ Advisor: Dr. Fernando L. Ponta
}

\begin{abstract}
Reducing the uncertainties related to blade dynamics by the improvement of the quality of numerical simulations of the fluid structure interaction process is a key for a breakthrough in wind-turbine technology. A fundamental step in that direction is the implementation of aeroelastic models capable of capturing the complex features of innovative prototype blades, so they can be tested at realistic full-scale conditions with a reasonable computational cost.

We make use of a code based on a combination of two advanced numerical models implemented in a parallel HPC supercomputer platform: First, a model of the structural response of heterogeneous composite blades, based on a variation of the dimensional reduction technique proposed by Hodges and Yu. This technique has the capacity of reducing the geometrical complexity of the blade section into a stiffness matrix for an equivalent beam. The reduced 1-D strain energy is equivalent to the actual 3-D strain energy in an asymptotic sense, allowing accurate modeling of the blade structure as a 1-D finite-element problem. This substantially reduces the computational effort required to model the structural dynamics at each time step. Second, a novel aerodynamic model based on an advanced implementation of the BEM (Blade Element Momentum) Theory; where all velocities and forces are re-projected through orthogonal matrices into the instantaneous deformed configuration to fully include the effects of large displacements and rotation of the airfoil sections into the computation of aerodynamic forces. This allows the aerodynamic model to take into account the effects of the complex flexo-torsional deformation that can be captured by the more sophisticated structural model mentioned above.

In this thesis we have successfully developed a powerful computational tool for the aeroelastic analysis of wind-turbine blades. Due to the particular features mentioned above in terms of a full representation of the combined modes of deformation of the blade as a complex structural part and their effects on the aerodynamic loads, it constitutes a substantial advancement ahead the state-of-the-art aeroelastic models currently available, like the FAST-Aerodyn suite. In this thesis, we also include the results of several experiments on the NREL-5MW blade, which is widely accepted today as a benchmark blade, together with some modifications intended to explore the capacities of the new code in terms of capturing features on blade-dynamic behavior, which are normally overlooked by the existing aeroelastic models.
\end{abstract}




\section{INTRODUCTION}

The increasing worldwide energy demand and the need to reduce the environmental impact caused by conventional electricity generation technologies have driven a re-emergence of interest in wind energy over the past decades. Nowadays wind energy is an important supplier of grid-connected electricity in the global energy picture thanks to considerable technological progress during the last twenty years [1]. Wind power is one of the most rapidly growing renewable energies. Global installed capacity grew from 14,604 $M W$ in 2000 to $84,934 M W$ in 2007, an impressive rate of $482 \%$ in only seven years according to [2]. By the end of 2009 the produced energy was $340 T W h$ according to the World Wind Energy Association (WWEA) [3]. This generated power is about 2\% of the worldwide electricity generation, which clearly shows how the global installation capacity of wind power accelerates. Updated sources reflect a total installed power of $238.3 \mathrm{GW}$ worldwide by the end of 2011 [4]. Europe and the US are the regions where the wind industry is mainly concentrated; nevertheless developing countries such as China, Brazil and India show signs of a sustained emerging of this industry.

During the last thirty years, increasing the size of the state-of-the-art machine has been the spontaneous tendency in the wind-turbine industry [5]. Economies-of-scale factors drive this tendency substantially reducing the cost of wind energy. State-of-the-art wind turbines have an output power ranging between 3.6 to $7.5 \mathrm{MW}$. GE, RE-Power, Vestas, Siemens, and Enercon are the main manufacturers of this type of machine. Figures 1.1a and 1.1b show the REpower 5M and the Enercon E-126 respectively, two of the best examples of multi-megawatt commercial wind turbines available on the market today. The REPower $5 \mathrm{M}$ can generate $5 \mathrm{MW}$ with a rotor diameter of $126 \mathrm{~m}$ (see basic characteristics on table 1.1) being available for both inland and offshore installations. On the other hand, the Enercon E-126 was conceived for inland installations only and, according to the manufacturer [6], this wind turbine is able to generate 7.5 MW with a rotor diameter of 127 $m$.

Industry insiders have been talking in recent years about a next-generation of giant offshore turbines of up to $12 \mathrm{MW}$ with rotor diameters up to $200 \mathrm{~m}$ [7]. This next-generation of superturbines would imply a substantial reduction in wind-energy costs if successfully 


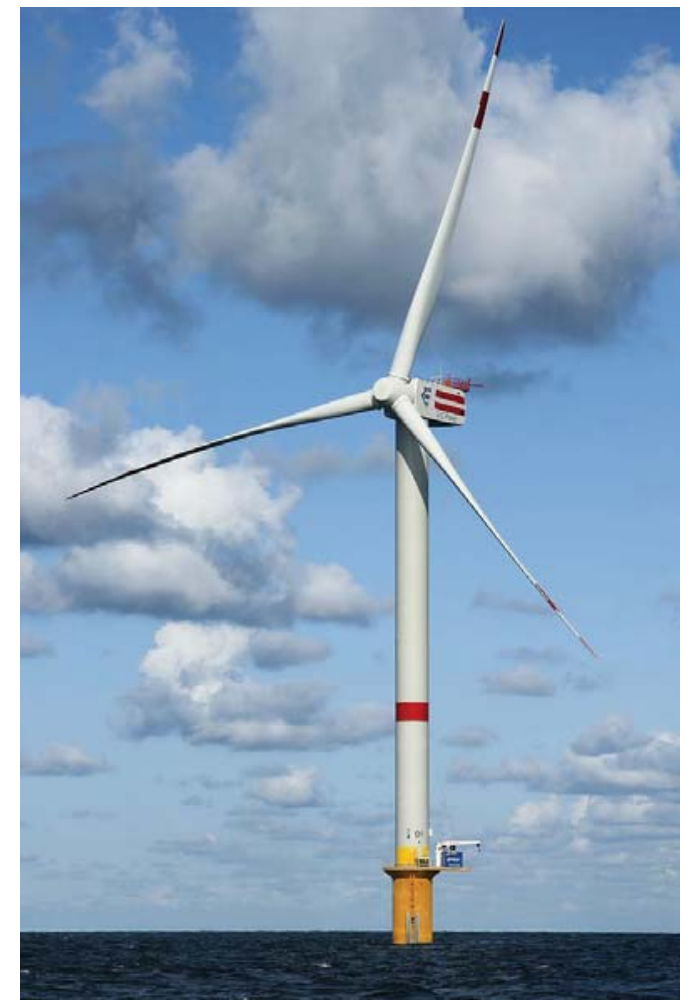

(a)



(b)

Figure 1.1. State-of-the art multi-megawatt wind turbines: (a) REpower 5M, with an output power of $5 \mathrm{MW}$ and a rotor diameter of $126 \mathrm{~m}$. Picture taken by Hans Hillewaert. (b) Enercon E-126, with an output power of $7.5 \mathrm{MW}$ and a rotor diameter of $127 \mathrm{~m}$. Picture from Jumanji Solar's photostream. Copyright statement in Appendix B.

Table 1.1

Main characteristics of the Repower 5MW wind turbine.

\begin{tabular}{ll}
\hline Design & \\
Rated Power & $5000 \mathrm{~kW}$ \\
Cut-In Wind Speed & $3.5 \mathrm{~m} / \mathrm{s}$ \\
Rated Wind speed & $13 \mathrm{~m} / \mathrm{s}$ \\
Cut-Out Wind Speed & \\
$\quad$ Offshore Version & $30 \mathrm{~m} / \mathrm{s}$ \\
$\quad$ Onshore Version & $25 \mathrm{~m} / \mathrm{s}$ \\
Rotor & $126 \mathrm{~m}$ \\
Diameter & $6.9-12.1 \mathrm{rpm}$ \\
Speed Range & \\
Blades & $61.5 \mathrm{~m}$ \\
Length & \\
\hline
\end{tabular}




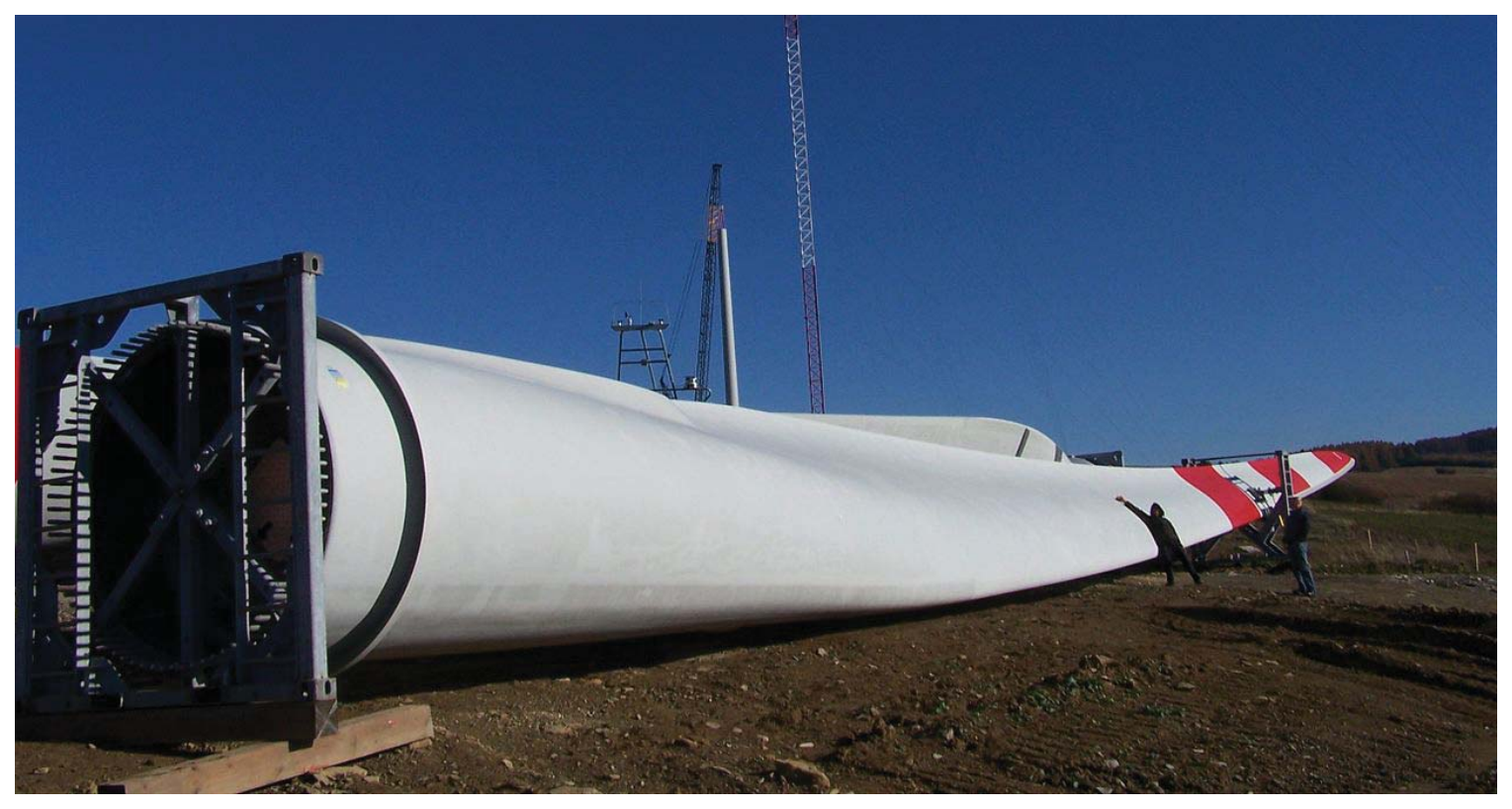

Figure 1.2. REPower $5 \mathrm{M}$ wind turbine blades. Wind farm installation at Bukowsko-Nowotaniec, Poland. Picture taken by Korona B. Copyright statement in Appendix B.

developed. Added to this, Chen et al. [1] suggest that, in favorable sites, it might be feasible to produce hydrogen competitively as a substitute fuel. This would help to close the existing gap between global fuel demands and the maximum amount of biofuel that may be sustainably produced.

Technologically, wind turbines evolved from the fixed-speed stall-controlled conception to the modern variable-speed and pitch-controlled turbines [8]. Today's market offers interesting combinations of innovative concepts with proven technology for both generators and power electronics [9]. However, there still exist several technological challenges to overcome in the development of wind power. Some of these involve reducing generation costs at utility scales in both inland and offshore locations with upscaled wind turbines that cost less to build than current models [1].

While the rest of the wind-turbine subsystems are highly developed technological products, blades are unique; no other technological application uses such a device. Therefore, upscaling these components not only presents huge challenges from the design standpoint and the complex non-linear aeroelastic response, but also from the manufacturing and transport logistics (see figure 1.2). The analysis of wind turbine blades constitutes a challenging problem in an emergent technological field which has a significant impact for the society.

Current wind-turbine blade technology based on composite laminates is labor-intensive and requires a highly-skilled workforce, creating a critical bottleneck in terms of industrial 


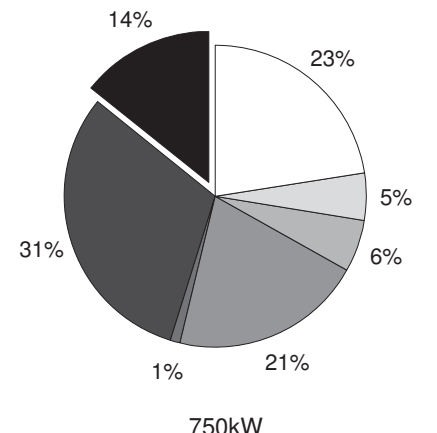

$750 \mathrm{~kW}$

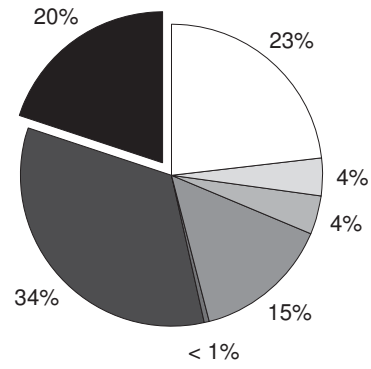

$1500 \mathrm{~kW}$

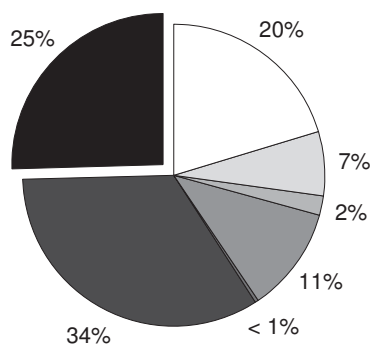

$3000 \mathrm{~kW}$

Figure 1.3. Proportional cost of wind-turbine subsystems (according to [5]). Blades and rotor comprise up to $25 \%$ of a wind turbine's total cost.

workforce and infrastructure that hampers a rapid expansion of wind-energy. It also poses a barrier to turbine upscaling that reflected in the increasing share of the cost of the rotor as turbine size increases (see figure 1.3).

The structural conception of today's blades also poses huge challenges in terms of transport logistics and crane capacity. Transportation cost increases as blades grow in length. The risk of damage during transportation, and hence, the cost of insurance, also increases with length. Moreover, while the rest of the turbine subsystems may be treated as modules assembled on site, blades are one-piece monolithic components, substantially complicating transport logistics (see figure 1.4). Limitations in crane capacity are the other critical factor to take into account during the turbine assembly phase. Thus, transport and lifting logistics may impose a premature limit for turbine upscaling, even before the actual limits in blade length for the current manufacturing technology are reached.

Blades operate under a complex combination of fluctuating loads, and huge size differences complicate extrapolation of experimental data from the wind-tunnel to the prototype scale. Oscillations and deformation of blades during operational stages are intimately related to the process of vortex formation and shedding in their wakes. This, in turn, determines the fluctuations in the aerodynamic forces acting on the blades creating a cyclic process of fluid-structure interaction. At design stages of blades and other aerodynamic surfaces, understanding and modelling these complex interactions is crucial. Hence, computer models of fluid-structure interaction phenomena are particularly relevant to the design and optimization of wind-turbines.

The wind-turbine industry is increasingly using computer models for blade structural design and for the optimization of its aerodynamics. Nevertheless, the complex interaction of physical processes that characterize the coupled aeroelastic problem still exceeds the 


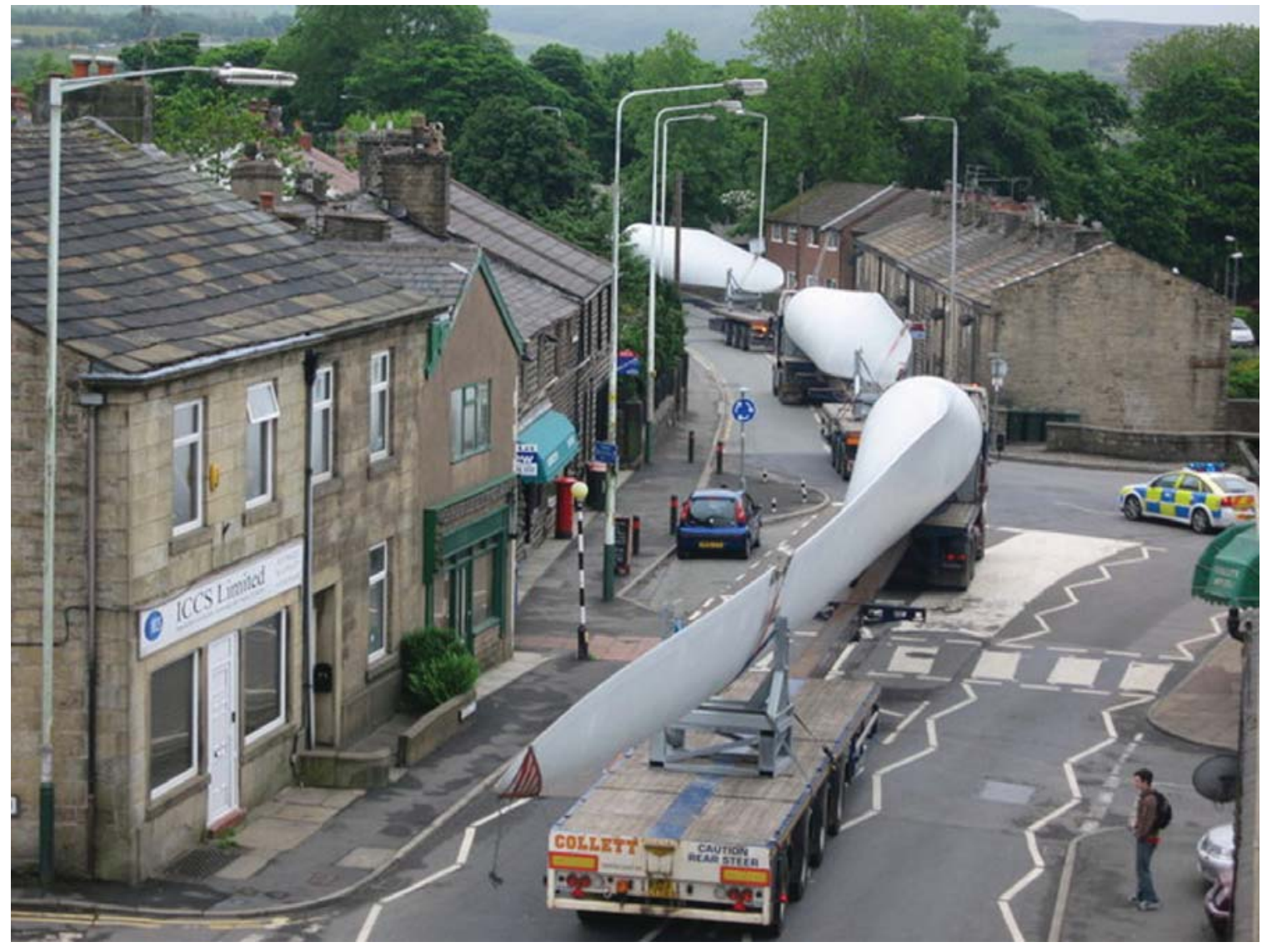

Figure 1.4. Wind-turbine blades convoy on the way to Scout Moor wind farm, England. Picture taken by Paul Anderson. Copyright statement in Appendix B. 
capacities of existing commercial simulation codes. The result is a very understandable tendency of the industry to be cautious with the introduction of new concepts in order to ensure reliability. Innovations are likely to introduce changes in structural response and may possibly require different control strategies, which would represent a major factor to take into account if the development of a new prototype blade is considered.

\subsection{Dissertation goals}

To allow for the simultaneous analysis of the aeroelastic problem together with any innovative control strategy into a single computationally efficient self adaptive algorithm, a series of algorithms will be implemented combining advanced numerical models to be executed in a parallel HPC supercomputer platform. A model of the structural response of heterogeneous composite blades using Variational Asymptotic Beam Sectional techniques will be used to reduce the geometrical complexity of the blade section and/or its material inhomogeneousness into a stiffness matrix for an equivalent 1-D beam. The equations of motion for the 1-D finite-element problem of the equivalent beam are solved using a nonlinear adaptive Ordinary Differential Equation (ODE) solver. This type of solver is based on variable-timestep/variable-order ODE algorithms that check the solution by monitoring the local truncation error at every timestep, improving the efficiency and ensuring the stability of the time-marching scheme.

This structural model will be combined eventually with three flow models that differ in the level of description they provide and the computational resources they require. In this dissertation work, we will develop and implement the first level of description for modeling the flow, Level-1, which involves modeling the rotor wake aerodynamics with the so-called Blade Element Momentum theory (BEM), which constitutes the base of several well-known codes like AeroDyn [10,11]. The next two levels (Levels 2 \& 3) would involve a full simulation of the dynamics of the unsteady separated flow around the blade sections, which are going to be developed in future thesis works.

The use of a nonlinear adaptive ODE algorithm provides a common framework to control the time-dependant solution for the aeroelastic problem. Equations modeling the dynamics of the control system and electromechanical devices may also be added to the general ODE system, with the control actions and the electrical network dynamics modifying the boundary conditions for the aeroelastic solution. This provides a natural way of integrating the structural response and the dynamics of the control system into the general solution of the flow problem in a process constantly controlled by the same self-adaptive ODE algorithm. 
The purpose of this dissertation work is two-fold:

- First, to develop the computational tools to implement the above-mentioned Common $O D E$ framework to control the interaction of the computational modules modeling the behavior of the different physical phenomena involved, as well as preparing a flexible interface for the incorporation of new modules that may be added in the future, or elaborated versions of existing ones.

- Second, to develop and implement an improved version of the BEM theory approach in the aerodynamic flow model, running complete simulations of the aeroelastic problem for different types of aerodynamic and structural designs.

\subsection{Dissertation Outline}

Chapter 2 presents the set of algorithms named the Common ODE framework and its implementation to control the interaction of the computational modules modeling the behavior of the different physical phenomena involved. As previously mentioned, one of the main advantages of this implementation is that the whole process is constantly controlled by the same self-adaptive ODE algorithm monitoring the local truncation error at every timestep, improving the efficiency and ensuring the stability of the time-marching scheme. The general structure of the code is written in MATLAB. Provided the universality and flexibility of this language, one can conceive modules for the different problems involved (flow, structure, control-system dynamics, etc.) assuring inter-compatibility among them. The modules may be treated individually, interfacing with the common ODE routine. Contrary to a monolithic approach, this modular design substantially simplifies further development of the code by the improvement of each submodel independently, opening the door for the simultaneous analysis of the aeroelastic problem together with any innovative control strategy into a single computationally-efficient self-adaptive algorithm.

In chapter 3 we present the theoretical basis of the Level-1 flow model. This flow model will provide the aerodynamic loads along the rotor blades, being sensitive enough to take advantage of all the complex deformation modes that the structural model is able to provide. At the same time, the feedback of the flow model will need to be at the same level of description as the structural model to accurately reflect the fluid-structure interaction on the rotor blades. The Level-1 flow model is based on a complete reformulation of the BEM theory, incorporating linear operators that perform rotations of the physical magnitudes involved (velocities, forces, etc.) to take into account the large sectional rotations of blade elements out of the rotor's plane. Added to this, the Level-1 incorporates, through its Flow Model Interphase (FMI), the ability to correct the aerodynamic coefficients data of the different airfoils to incorporate 3-D effects in real-time. Another advantage of this flow model 
is that it can handle multiple data tables for the different airfoils and use them according to the instantaneous aerodynamic situations. This allows for unique features like using multiple Reynolds data tables or the possibility to include aerodynamic coefficients data from modified airfoils with active control surfaces as micro-tabs or deployable spoilers, all in real-time. The Level- 1 flow model overcomes multiple limitations that the existing computational codes based on the classical BEM theory present.

Chapter 4 presents the structural model implemented for the study of aeroelastic simulations, a generalized Timoshenko code developed by Dr. Otero [12,13] based on a modified implementation of the Variational-Asymptotic Beam Sectional (VABS) model proposed and developed by Prof. Hodges and his collaborators [14]. This model, which is able to work with curved and twisted composite beams, uses the same variables as classical Timoshenko beam theory, but the hypothesis of beam sections remaining planar after deformation is abandoned. Instead, the real warping of the deformed section is interpolated by a 2-D finite-element mesh and its contribution to the strain energy is put in terms of the classical 1-D Timoshenko's variables by means of a pre-resolution. The geometrical complexity of the blade section and/or its material inhomogeneousness are reduced into a stiffness matrix for the 1-D beam. The reduced 1-D strain energy is equivalent to the actual 3 -D strain energy in an asymptotic sense. Elimination of the ad hoc kinematic assumptions produces a fully populated $6 \times 6$ symmetric matrix for the 1-D beam, with as many as 21 independent stiffnesses, instead of the six fundamental stiffnesses of the original Timoshenko theory [15]. That is why it is referred to as a generalized Timoshenko theory. Even for the case of large displacements and rotations of the beam sections, this model allows for accurate modeling of the bending and transverse shear in two directions, extension and torsion of the blade structure as a 1-D finite-element problem. Thus, we are able to decouple a general 3-D nonlinear anisotropic elasticity problem into a linear, 2-D, cross-sectional analysis (that may be solved a priori), and a nonlinear, 1-D, beam analysis for the global problem, which is what is needed at each timestep of a fluid-structure interaction analysis.

In chapter 5 we present the analysis of the numerical experimentation results. To explore the possibilities of our computational codes we propose to use a set of rotor blades based on the 5-MW Reference Wind Turbine (RWT) project developed by NREL [16]. Based on the REpower 5M wind turbine, NREL's RWT was conceived for both inland and offshore installations and it is well representative of typical utility-scale multi megawatt commercial wind turbines. As a part of our numerical experimentation, and using the principles of Adaptive Blades, we take full advantage of the coupled deformation modes that our aeroelastic code can represent and analyse in this chapter different blade configurations optimizing the design to reduce extreme loads and improve fatigue performance. Flexo-torsional or bend-twist coupling is a concept originally coming from aeronautics. Fine tuning of the relative positions between the structural torsional center of the blade section, and its aerodynamic center (conventionally located at the quarter-chord length for typical airfoils) is one of the main principles of swept wings to avoid the so-called struc- 
tural divergence and self-adapt angles of attack on the wing sections. Another novel set of experiments in this chapter allow us to demonstrate the effects of gravitational forces on the blades and the way these computational tools could be used to address potential problems to successfully achieve the development of the next-generation upscaled giant wind turbines.

Finally, chapter 6 presents the conclusions for this dissertation work as well as the outlook for further work based on the analysis of the material presented in the previous chapters. 


\section{COMMON ODE FRAMEWORK}

To allow for the simultaneous analysis of the aeroelastic problem together with any innovative control strategy into a single computationally efficient self-adaptive algorithm, a series of computational codes were implemented combining advanced numerical models to be executed in parallel HPC supercomputer platforms. In this chapter we present this set of algorithms named the Common ODE framework and its implementation to control the interaction of the computational modules modeling the behavior of the different physical phenomena involved. We will start by describing the basis of this common ODE framework and the global interactions among the modules. In the following sections we will cover its numerical implementation and its flexibilities including the possibility to incorporate future modules or elaborated versions of existing ones.

For the structural module, a Generalized Timoshenko Beam Model (GTBM), which will be described in detail under chapter 4 , is used to reduce the geometrical complexity of the blade section and/or its material inhomogeneousness into a stiffness matrix for an equivalent 1-D beam. The equations of motion for the 1-D finite-element problem of the equivalent beam are solved using a non-linear adaptive ODE solver. This type of solver is based on variable-timestep/variable-order ODE algorithms that check the solution by monitoring the local truncation error at every timestep, improving the efficiency and ensuring the stability of the time-marching scheme.

This structural module will ultimately be combined with any of three alternative flow models that differ in the level of description they provide and the computational resources they require. Figure 2.1 shows a pseudo-code diagram of this global scheme indicating the interrelation between the dynamics of the structure, flow, control systems and other devices. The use of a nonlinear adaptive ODE algorithm provides the common framework the ability to control the time-dependant solution for the aeroelastic problem.

The general structure of the code is written in MATLAB. Provided the universality and flexibility of this language, one can conceive modules for the different problems involved (flow, structure, control-system dynamics, etc.) assuring inter-compatibility among them. The modules may be treated individually, interfacing with the common ODE routine. 


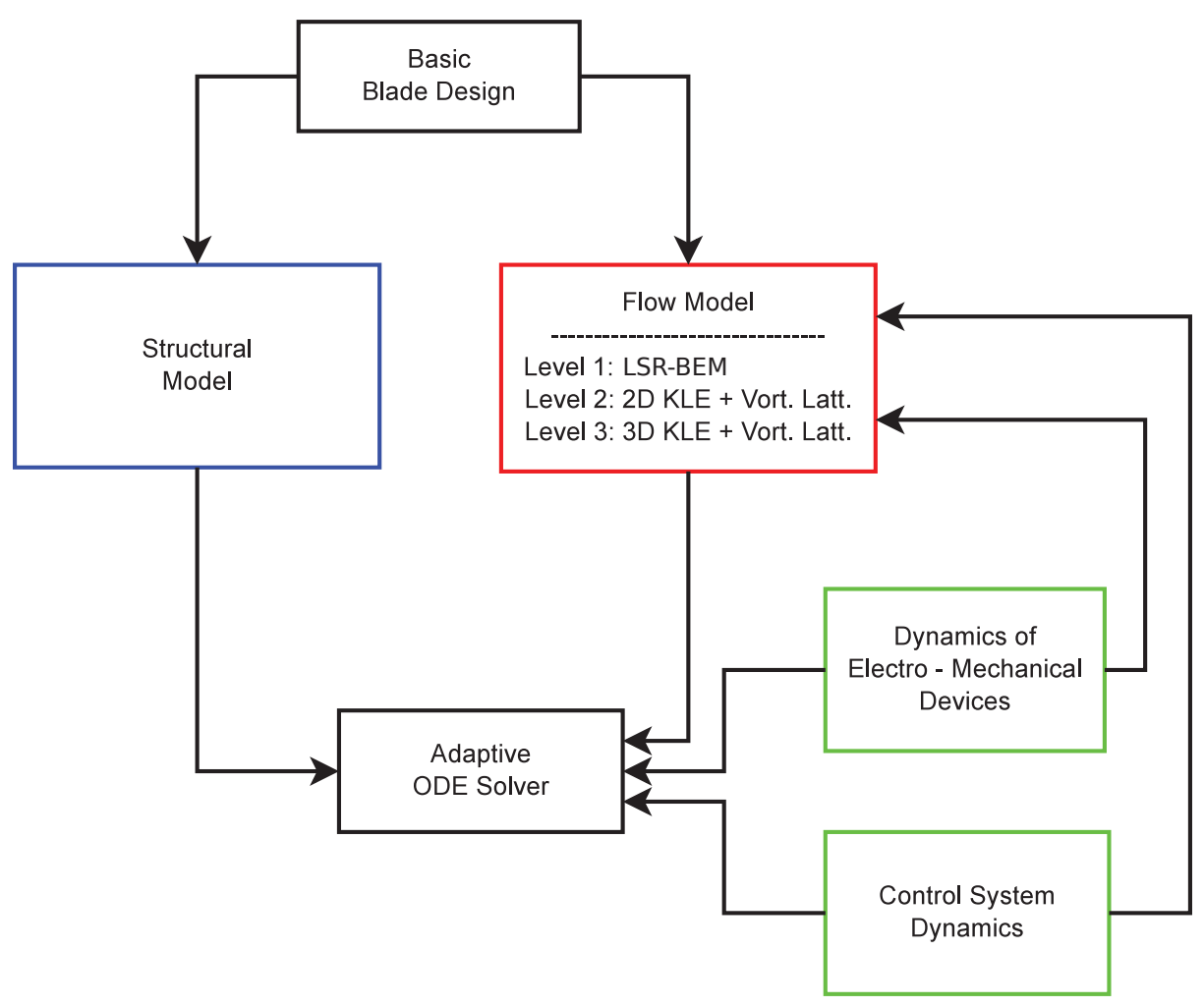

Figure 2.1. Pseudo-code diagram.

Contrary to a monolithic approach, this modular design substantially simplifies further development of the code by the improvement of each submodel independently, opening the door for the simultaneous analysis of the aeroelastic problem together with any innovative control strategy into a single computationally efficient self-adaptive algorithm.

\subsection{Numerical implementation}

In this section we will show the numerical implementation of the common ODE framework and how the nonlinear adaptive ODE algorithm provides the natural means to integrate the different modules. Figure 2.2 shows the interrelation diagram of $n$ different computational modules divided in three main blocks: Parameters input, Main Framework and Post-processing.

The first block defines the problem in terms of the simulation timespan as well as the amount of intervening modules. Here, the modules are identified and their needed data is collected to then evaluate the different instances in the ODE problem. These basic instances 
are divided in: Setting of the Initial Conditions (IC) for the ODE problem, evaluations of the Right-Hand-Side (RHS) of the ODE system, and post-processing of accepted timesteps through output-functions. Along with these basic instances, we added the possibility to include the definition and evaluation of the analytical Jacobian matrix for each module in case an analytical form for the Jacobian exists. This possibility saves considerable computational time when the ODE solver needs to re-compute the Jacobian when numerical stiffness arises $[17,18]$. In MATLAB, in the absence of an analytical form, the default Jacobian computation is made numerically by a finite-difference approximation, where the computation time needed scales with the square of the number of degrees of freedom of the ODE problem [19].

The second block of figure 2.2, the Main Framework, represents the actual ODE solver and shows schematically how we interconnect the different intervening modules in the problem. After processing the information coming from the Parameters-input block, these parameters are distributed to the different sub-blocks for the corresponding computations. We see in the figure that each sub-block has a main function which administers the processed parameters to each defined module and then gathers all the solutions as one common output. Thus, the Initial IC sub-block will output to the common framework a vector with the concatenated ICs of all the modules. In the same way, the common framework will initialize the RHS evaluator with the IC vector computed previously. Now the main RHS evaluator function re-distributes the corresponding IC elements from the vector to the modules and solves for the current timestep. Of course, for subsequent timesteps, the main framework will provide previously-converged solutions of the ODE problem to keep advancing the time scheme. During the RHS evaluations, temporary data might be processed or stored. When the ODE solver accepts the solution for the current timestep, this information might also be physically stored in a hard drive. The solution data for each timestep are fed back into the different modules after being processed by the output functions. This inter-modular data sharing takes place naturally as part of the normal operation of the ODE algorithm. For example, the updated solution of the aerodynamic loads are fed back into the structural model. Also, part of this data could trigger control actions from an eventual control module which changes the blade pitch angle, actuates flow-control devices, or varies the rotational speed of the electro-mechanical train. As mentioned before, one of the main advantages of this implementation is that the whole process is constantly controlled by the same self-adaptive ODE algorithm monitoring the local truncation error at every timestep, improving the efficiency and ensuring the stability of the time-marching scheme.

The third block is self explanatory and shows the possibility of post-processing the data obtained, generating at each timestep reports for the different variables involved. There is also the possibility of monitoring intermediate variables besides the main ones, which constitutes a key feature to a better understanding of the complex interaction between the different physical mechanisms involved. 




Figure 2.2. Common ODE framework diagram. 


\subsection{The Level-1 flow model}

The first level of description for modeling the flow, Level-1, the one implemented in this work, involves modeling the rotor wake aerodynamics with the so-called Blade Element Momentum theory (BEM), described under chapter 3. In the future, the next two levels (Levels 2 and 3) would involve a full simulation of the dynamics of the unsteady separated flow around the blade sections using a new series of adaptive algorithms, based on the hybrid (or vorticity-velocity) formulation of the Navier-Stokes equations. The kinematic Laplacian equation (KLE) technique will be used to create a complete decoupling of the two hybrid variables in a vorticity-in-time/velocity-in-space split approach, which results in a numerical scheme written in terms of an ODE set [20].

The equations modeling the dynamics of the control system and electromechanical devices may also be added to the general ODE system, with the control actions and the electrical network dynamics modifying the boundary conditions for the aeroelastic solution. This provides a natural way of integrating the structural response and the dynamics of the control system into the general solution of the flow problem in a process constantly controlled by the same self-adaptive ODE algorithm. In the future, the possibility of integrating submodels for other physical phenomena could be explored.

Among the main modules shown in figure 2.1, the one for the Basic Blade Design serves to define not only the aerodynamic parameters for the rotor's normal operational conditions, but also the structural features resulting from the blade-section's internal layout, selection of materials, manufacturing procedures, and eventual flow-control devices that may be added. Figure 2.3 shows a detailed pseudo-code diagram with the different subroutines inside each module and their interrelation. The structural module generates the ODE equations on an equivalent 1-D beam as will be explained in section 4.2. Dimensional reduction from the actual 3-D blade structure to the 1-D equivalent problem is an essential part of this module. The concentrated and distributed forces in the blade needed for the evaluation of the right hand side of this ODE set will come from the evaluation of the flow module and from the control-system actuators. The dynamical response of the electromechanical train initially provides the time-dependant value for the rotational speed of the main shaft using as an input the driving torque from the rotor. This value is also used to compute the incoming flow on the blade sections, as will be seen under section 3.3. More sophisticated interactions could be achieved in the future by linking the electro-mechanical and the control-system modules.

Preliminary information about the structural response is provided, at relatively low computational cost, by linearizing the equations for the equivalent 1-D beam and getting an aeroelastic steady-state solution for a certain set of operational parameters (see Fig. 2.3).

The frequencies and linear vibrational modes for that deformed configuration can also be 
obtained by solving an eigenvalue problem around that linearised steady-state solution. The latter is a valuable by-product of the dimensional-reduction technique that may be advantageously applied at the initial stages of the design of both the blade structure and the control system. 




Figure 2.3. Pseudo-code diagram for the common framework on the Level-1 flow model option. 


\subsection{The stiff multistep BDF-ODE solver}

The adaptive-stepsize technique is employed to control the accuracy of the simulations and to enhance their efficiency to provide stable steady state or transient solutions. The adaptive timestep algorithms usually use approximate values of the local truncation error to estimate the optimal timestep, and some of them are even based on some kind of "Thumb Rules". In some of the standard algorithms, the user is required to specify the accuracy requirement on local truncation error which is compared with computed values of the same which should be within accepted accuracy or tolerance range [21].

Backwards-Differentiation formula (BDF) ODE implements a constant monitoring of the rate of convergence [22]. If the code predicts that convergence for the actual timestep will not be achieved in four steps, it could stop the iteration process. Thus, if the previously computed approximation for the Jacobian is no longer valid, a new Jacobian is formed. Otherwise, the integration stepsize is reduced. Optimizations for reusing the Jacobian in BDF-ODE minimize the involved recalculation times allowing BDF-ODE to compete rather well with solvers for non-stiff problems while providing the needed tools to overcome eventual stiffnesses [23].

In this work, a BDF solver with adaptive stepsize control has been used successfully. The solver ODE15s from the MATLAB ODE suite was adopted for the common ODE framework and used for the aeroelastic experiments.

The ODE15s is a variable order, variable stepsize implementation of the numerical differentiation formulas (NDF) in terms of backward differences [23] which also allows integration using BDFs. The integration order in ODE15s can be altered from 1 to 5 [23], which gives it a powerful capability in terms of adapting itself to a variety of complex multiphysics situations. Being one of the so-called "methods with memory", it is particularly efficient in cases where the evaluation of the RHS of the ODE system is computationaly intensive. By using the solution at previous timesteps, the number of evaluations of the RHS required for a certain order of accuracy is usually reduced substantially. For more details about ODE15s in MATLAB, original research paper by L.F. Shampine and M.W. Reichelt [23] can be referred.

The rationale behind using ODE15s for the experimentation lies in its effectiveness dealing with the stiffness originated from complex aeroelastic interactions. 


\section{THE LEVEL-1 FLOW MODEL}

Here, we present the theoretical basis of the Level-1 flow model that will interact with its structural counterpart. This flow model will provide the aerodynamic loads along the rotor blades, being sensitive enough to take advantage of all the complex deformation modes that the structural model is able to provide. At the same time, the feedback of the flow model will need to be at the same level of description of the structural one to accurately model the fluid-structure interaction on the rotor blades. At the moment of writing this dissertation, the state-of-the-art computational code to compute aerodynamic loads for wind turbine rotors is the one developed by the National Renewable Energy Laboratory (NREL), Aerodyn [10,11]. As in Aerodyn, the basis for our aerodynamic flow model will be the well known Blade Element Momentum theory (BEM). Nevertheless, due to the high level of detail that our structural model can provide, a complete reformulation was needed for the aerodynamic model overcoming multiple limitations that the existing computational code presents [24].

We will begin this chapter with a section introducing the classic formulation of the BEM theory in its particular application to Horizontal Axis Wind Turbines (HAWT), followed by another section describing our new formulation designed to overcome the limitations of the classic theory.

\subsection{Theoretical Background: The Classic Blade Element Momentum theory}

The BEM theory, generally attributed to Betz and Glauert [25], actually originates from two different theories: blade element theory and momentum theory. Blade element theory is associated with the description of aerodynamic forces, lift and drag, generated on an airfoil shape as the result of its interaction with an incoming wind current. Momentum theory is related to the conservation of linear and angular momentum on a control volume, generally modeled as a stream tube, analysing the forces on the rotor blades [26]. 
Let's now review the aerodynamics of an airfoil shape to identify and describe the aerodynamic loads acting on the blade. More details about Rotor Blade theory and BEM theory can be found in [26] and [27].

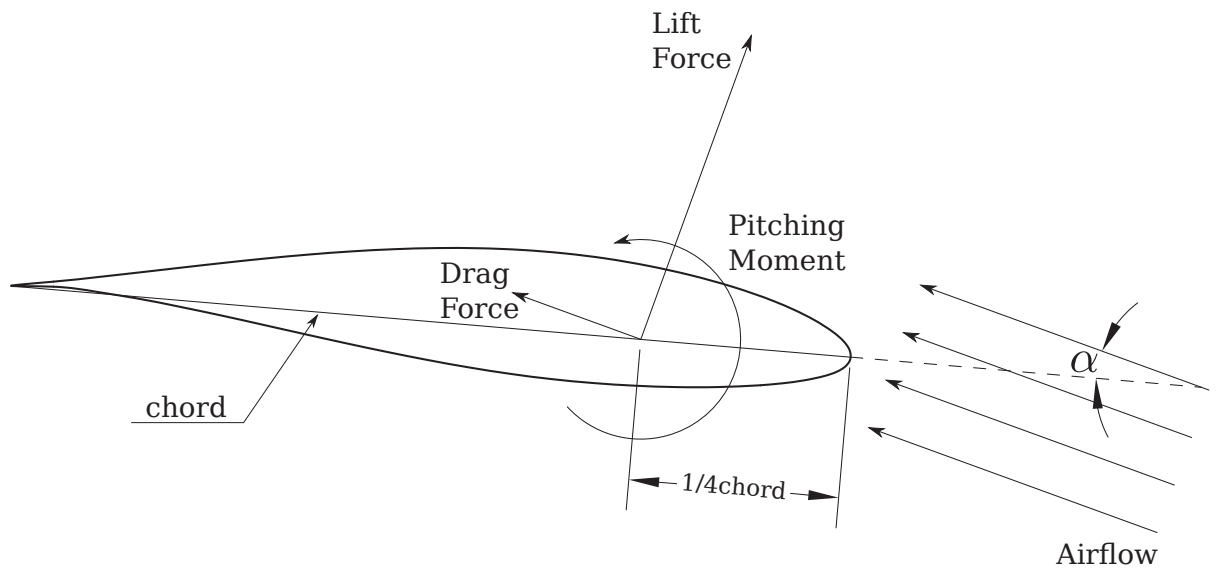

Figure 3.1. Aerodynamic efforts on an airfoil.

Fig. 3.1 shows an airfoil and the main aerodynamic efforts resulting from an incoming airflow. The chord is defined as the line connecting the leading edge and the tip of the airfoil. The angle between the incoming airflow and the chord is called angle of attack and is denoted by $\alpha$. The resultant of all of the pressure and friction forces acting along the airfoil are shown in the picture as two forces and a moment reduced at a distance of $c / 4$ from the leading edge. The Lift force is a consequence of the unequal pressure on the upper and lower airfoil surfaces and its direction is perpendicular to the oncoming airflow direction. The Drag force is mainly due to viscous friction forces at the surface of the airfoil and its direction is parallel to the direction of oncoming airflow. The real acting point of this forces depends on the angle of attack $\alpha$. The reduction of the aerodynamic forces to $c / 4$ is the cause of the Pitching moment, which its acting direction is defined to be perpendicular to the airfoil cross-section. Lift coefficient $C_{l}$, drag coefficient $C_{d}$ and pitching moment coefficient $C_{m}$ are the non-dimensional definitions for the aerodynamic efforts:

$$
C_{l}=\frac{f_{l}}{\frac{1}{2} \rho U^{2} c} ; \quad C_{d}=\frac{f_{d}}{\frac{1}{2} \rho U^{2} c} ; \quad C_{m}=\frac{m}{\frac{1}{2} \rho U^{2} c^{2}},
$$

where: $f_{l}$ and $f_{d}$ are the lift and drag forces, $m$ is the distributed moment per unit length, $\rho$ is the density of air, $U$ is the velocity of undisturbed airflow, and $c$ is the airfoil chord length.

There is another wind component added to the oncoming airflow at the airfoil due to the rotation of the blade. Moreover, due to the presence of the rotor, the airflow loses speed 
and a vortex wake is formed behind it (see figure 3.2, from [27]). Figure 3.3 shows a wind turbine airfoil cross-section under working conditions. Here, all acting velocities, forces, and angles are represented. The entities in this figure are: $\Omega$, which indicates the magnitude of the angular velocity and whose direction coincides with the rotor's rotational axis. The incoming wind is parallel to the rotor's axis and has a magnitude of $U(1-a)$, where $U$ is the velocity of the unperturbed airflow and $a$ is the axial induction factor, which indicates the velocity loss due to the presence of the rotor. The represented airfoil section is at a distance $r$ of the rotor axis and its tangential velocity will be $\Omega r$.

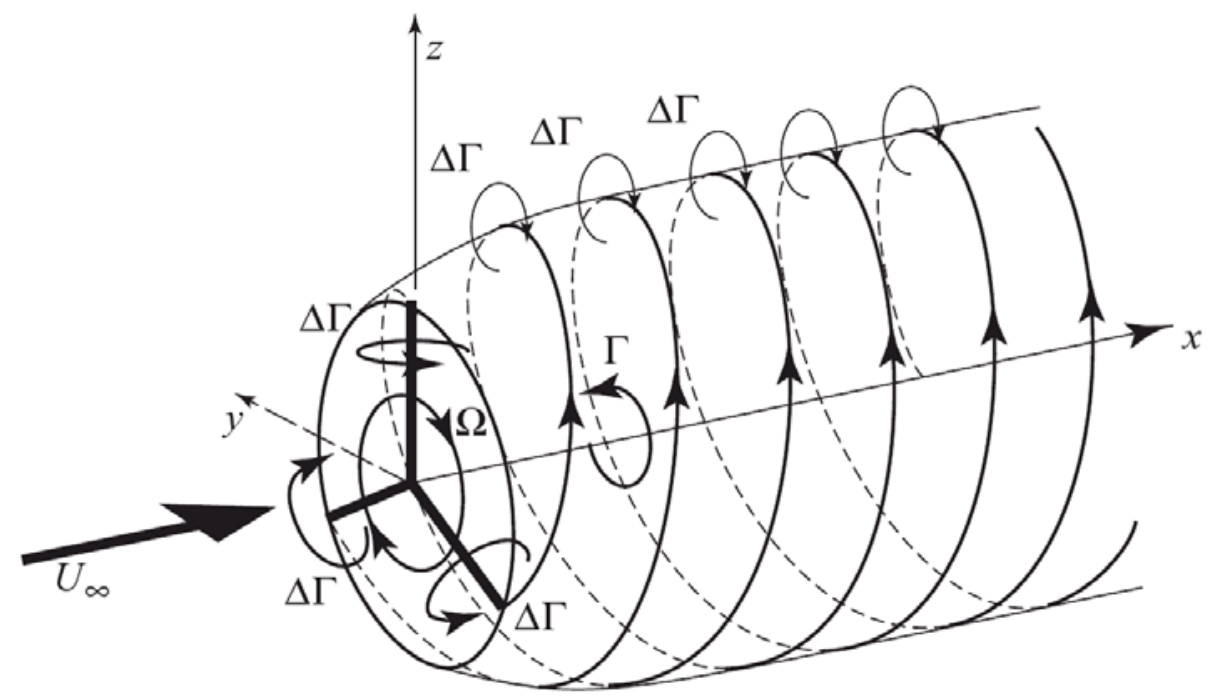

Figure 3.2. Helical vortex wake shed by rotor with three blades at uniform circulation [27]. Copyright statement in Appendix B.

We will define now the tip speed ratio as the ratio of the rotor's tangential velocity and the free stream velocity of the incoming wind,

$$
\lambda=\frac{\Omega R}{U},
$$

where $R$ is the rotor's radius. Analogously, the speed ratio depends on the radial distance $r$ to the considered airfoil section on the rotor axis.

$$
\lambda_{r}=\frac{\Omega r}{U}=\lambda \frac{r}{R}
$$

Due to the rotation of the wake behind the rotor (see figure 3.2, from [27]), the relative wind velocity on the airfoil is: 


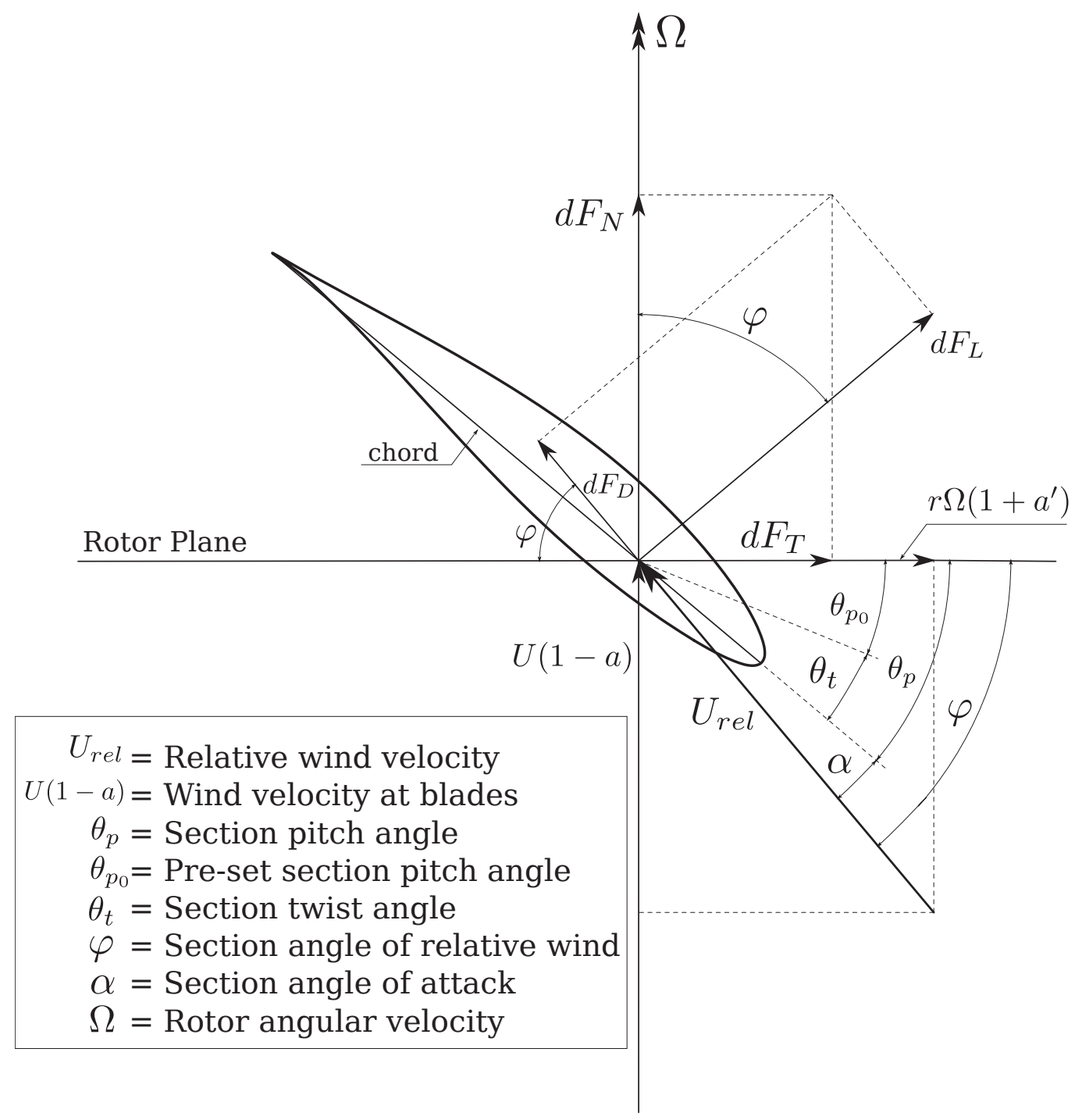

Figure 3.3. Top view analysis of an HAWT blade cross-section.

$$
U_{r e l}=\sqrt{(U(1-a))^{2}+\left(\Omega r\left(1+a^{\prime}\right)\right)^{2}},
$$

where $a^{\prime}$ is the angular induction factor which represents the increment on the relative tangential velocity in the blade's cross-section due to the rotation of the wake behind the rotor.

The angle $\varphi$ between the relative wind velocity and the blade's plane of rotation is called angle of relative wind. The angle of attack $\alpha$ is defined between the chord line and the relative wind. The difference of the relative wind angle and the angle of attack is called 
the section pitch angle $\theta_{p}$. This angle is also the summation of the blade pitch angle $\theta_{p 0}$ and the section twist angle $\theta_{t}$. Figure 3.3 also defines $d F_{l}$ and $d F_{d}$, the incremental lift and drag forces. $d F_{N}$, an incremental force normal to the plane of rotation is responsible for the rotor thrust. $d F_{T}$, tangential to the circle swept by the rotor, creates the torque which will be transformed into power at the shaft.

The basis for BEM theory relates the force of a blade element with the change of momentum of the air which passes through the annulus swept by the element [27] (see figure 3.4, extracted from [27]). Thus, the radial interaction between the flows through contiguous annuli is neglected. This could be true only if the radially variation of the axial flow induction factor is zero. Nevertheless, it is demonstrated experimentally that this assumption of radial independence is acceptable. The theory provides an iterative process to compute the induction factors $\left(a, a^{\prime}\right)$ to then obtain the aerodynamic efforts acting on the rotor. Note that the process needs to be iterative as the induction factors depend on the angle of attack and wind relative angle for each blade section embedded in their lift and drag coefficients.

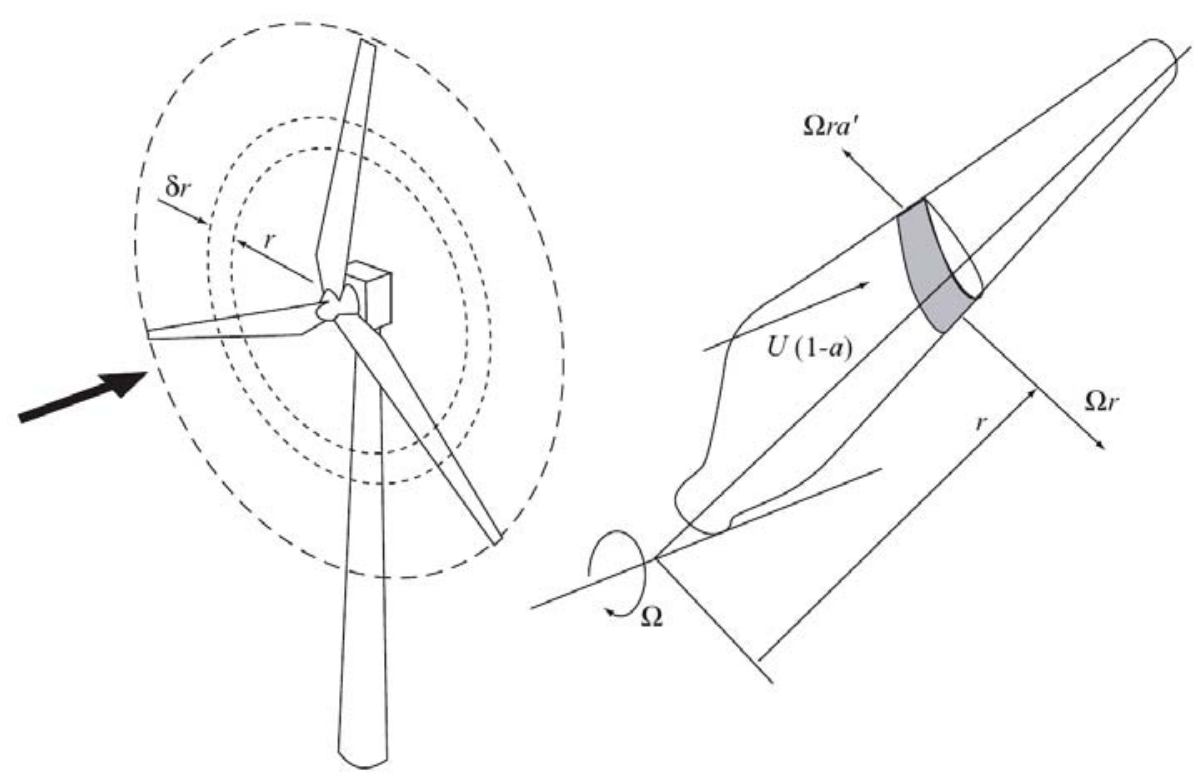

Figure 3.4. A blade element sweeping out an annular ring, from [27]. Copyright statement in Appendix B.

To begin with the procedure, we first define the blade solidity as the ratio of the total blade area and the rotor disc area:

$$
\sigma(r)=\frac{B c(r)}{2 \pi r}
$$


Using equation 3.5, we can now express the initial values for the induction factors for a blade section as:

$$
\begin{aligned}
& a_{0}(r)=\left[1+\frac{4 \sin ^{2}(\varphi(r))}{\sigma(r) C_{l \alpha_{o p t}}(r) \cos (\varphi(r))}\right]^{-1} \\
& a_{0}^{\prime}(r)=\left[\frac{4 \cos (\varphi(r))}{\sigma(r) C_{l \alpha_{o p t}}(r)-1}\right]^{-1} .
\end{aligned}
$$

The iterative computation process now follows for all the blade sections defined on our blades.

1. Obtain the angle of relative wind:

$$
\varphi_{i}(r)=\tan ^{-1}\left(\frac{1-a_{i-1}(r)+v_{o p}}{\left(1+a_{i-1}^{\prime}(r)\right) \lambda_{r}+v_{i p}}\right)
$$

where $v_{i p}$ and $v_{o p}$ are the in-plane and out-plane components of the structural deformation velocities of the blade section, coming form a structural model like for example FAST on the well known aeroelastic suite FAST-Aerodyn.

2. As a correction to the BEM theory to be applied to HAWTs, we need to take into account the effect of vortices being shed at the tip of the blade. To this end, we introduce a tip loss factor according to Prandtl [26], as follows:

$$
F_{t i p}=\frac{2}{\pi} \cos ^{-1}\left(\exp \left(-\frac{\frac{B}{2}\left(1-\frac{r}{R}\right)}{\frac{r}{R} \sin \left(\varphi_{i}(r)\right)}\right)\right) .
$$

In the same way, the presence of the hub near the root of the blade generates another vortex shedding which originates the addition of another correction factor to the induced velocity [27] as:

$$
F_{h u b}=\frac{2}{\pi} \cos ^{-1}\left(\exp \left(-\frac{B}{2}\left(\frac{r}{R}-R_{h u b} \frac{R}{r}\right) \sqrt{\frac{1+\lambda_{r}^{2}}{(1-a)^{2}}}\right)\right) .
$$

This corrections for tip and hub losses are then combined in a single correction factor as:

$$
F_{f}=F_{t i p} F_{h u b}
$$


We will come back to this later on a more detailed discussion about the needed modification for the BEM theory on HAWTs in the following sections.

3. Now, knowing the pitch angle for the blade section and using equation 3.8, we obtain the section's angle of attack:

$$
\alpha_{i}(r)=\varphi_{i}(r)-\theta_{p}(r)
$$

4. After computing $\alpha_{i}(r)$, we need to look for the lift, drag, and pitching moment coefficients $\left(C_{l i}(r), C_{d i}(r)\right.$ and $\left.C_{m i}(r)\right)$ for our blade section.

5. With this, we calculate the local thrust coefficient defined by:

$$
C_{T i}(r)=\frac{\sigma(r)\left(1-a_{i-1}(r)\right)^{2}\left[C_{l i}(r) \cos \left(\varphi_{i}(r)\right)+C_{d i}(r) \sin \left(\varphi_{i}(r)\right)\right]}{\sin ^{2}\left(\varphi_{i}(r)\right)},
$$

6. to then obtain the axial induction factor:

$$
a_{i}(r)=\left\{1+\frac{4 F_{i}(r) \sin ^{2}\left(\varphi_{i}(r)\right)}{\sigma(r)\left[C_{l i}(r) \cos \left(\varphi_{i}(r)\right)+C_{d i}(r) \sin \left(\varphi_{i}(r)\right)\right]}\right\}^{-1} ;
$$

7. and the angular induction factor:

$$
a_{i}^{\prime}(r)=\frac{\left(1-a_{i}(r)\right) \sigma(r)\left[C_{l i}(r) \sin \left(\varphi_{i}(r)\right)-C_{d i}(r) \cos \left(\varphi_{i}(r)\right)\right]}{4 F_{i}(r) \lambda_{r} \sin ^{2}\left(\varphi_{i}(r)\right)}
$$

8. After computing $a$ and $a^{\prime}$, we need to verify the convergence of $\left|a_{i}(r)-a_{i-1}(r)\right|<$ Tol and $\left|a_{i}^{\prime}(r)-a_{i-1}^{\prime}(r)\right|<$ Tol on every blade section for a given tolerance Tol.

Once convergence is achieved, given the local relative wind velocity,

$$
U_{r e l}(r)=\sqrt{\frac{U^{2}\left(1-a_{i}(r)\right)^{2}}{\sin ^{2}\left(\varphi_{i}(r)\right)}}
$$

where $i$ is the iteration of convergence, we can compute the lift and drag forces as well as the distributed pitching moment according to the following expressions:

$$
\begin{aligned}
& d F_{l}(r)=\frac{1}{2} \rho\left(U_{r e l}(r)\right)^{2} C_{l i}(r) c(r) \\
& d F_{d}(r)=\frac{1}{2} \rho\left(U_{r e l}(r)\right)^{2} C_{d i}(r) c(r) \\
& d M(r)=\frac{1}{2} \rho\left(U_{r e l}(r)\right)^{2} C_{m i}(r)(c(r))^{2} .
\end{aligned}
$$


Finally, using the converged angle of attack for the section, we need to decompose the distributed forces parallel and perpendicular to the direction of the airfoil's chord.

$$
\begin{gathered}
d F_{N}(r)=d F_{l}(r) \cos \left(\alpha_{i}(r)\right)+d F_{d}(r) \sin \left(\alpha_{i}(r)\right) \\
d F_{T}(r)=-d F_{l}(r) \sin \left(\alpha_{i}(r)\right)+d F_{d}(r) \cos \left(\alpha_{i}(r)\right) .
\end{gathered}
$$

These distributed forces called the Lilenthal forces, correspond to the coefficients of the same name. These forces are referred to the airfoil's chord and they differ from those shown in figure 3.3 (capital subindex) which are referred to the rotor plane.

\subsubsection{Corrective factors to BEM theory}

In order to apply this theory to HAWT rotors, we must introduce some corrective factors into the calculation process. BEM theory does not account for the influence of vortices being shed from the blade tips into the wake on the induced velocity field. These tip vortices create multiple helical structures in the wake which play a major role in the induced velocity distribution at the rotor [10]. To compensate for this deficiency in BEM theory, a tip-loss model originally developed by Prandtl is implemented as a correction factor to the induced velocity field [25],(see [26], [27] and equation 3.9. In the same way, a hub-loss model serves to correct the induced velocity resulting from a vortex being shed near the hub of the rotor, (see [26], [27] and equation 3.10.

Another modification needed in the BEM theory is the one developed by Glauert [28] to correct the rotor thrust coefficient. This correction plays a key role when the turbine operates at high tip speed ratios and the induction factor is greater than about 0.45 . At this point, basic assumptions of momentum theory become invalid and this correction is needed in order to take into account part of the flow in the far wake which starts to propagate upstream. Physically this flow reversal cannot occur and what actually happens is that more flow entrains from outside the wake increasing the turbulence. With this, the flow behind the rotor slows down but the thrust continues to increase [26,27].

BEM theory was originally conceived for axisymmetric flow. Often, however, wind turbines operate at yaw angles relative to the incoming wind, which produces a skewed wake behind the rotor. For this reason, the BEM model needs also to be corrected to account for this skewed wake effect [29,30].

The influence of the wind turbine tower on the blade aerodynamics is also modeled in the original AeroDyn [10]. It implements models developed by Bak et al. [31] and SRJ Powles [32] which provide the influence of the tower on the local velocity field at all points around the tower. This model accounts for increases in wind speed around the sides of the 
tower and the cross-stream velocity component in the tower near flow field.

\subsubsection{Limitations of the classic BEM theory for large deformations}

The tendency in the wind-turbine industry to increase the size of the state-of-the-art machine [16] drives not only to bigger, but also to more flexible blades which are relatively lighter. It is observed for this type of wind turbine blades that big deformations, either due to blade flexibility or to pre-conforming processes, produce high rotations of the blade sections. Moreover, blades could be pre-conformed with specific curvatures given to any of their axis (i.e. conning/sweeping). This tendency puts in evidence one of the most important limitations of the current BEM theory. While the basics of this theory keeps being perfectly valid, the actual mathematical formulation implies the assumption of blade sections remaining perpendicular to an outwards radial line contained in the plane of the actuator disk coincident with the rotor's plane. That is, even though the basics of the BEM theory (i.e. the equation of the aerodynamic loads and the change of momentum in the streamtubes) remains valid, the mathematical formulation cannot represent large rotations of the blade sections. This basically leads to a misrepresentation of the effects of the large deformation associated with flexible blades on the computation of the aerodynamic loads. Hence, a new mathematical formulation is required to project the velocities obtained from momentum theory onto the blade element's plane and then re-project backwards the resulting forces from Blade Element theory onto the plane of the stream tube actuator disk.

When analysing BEM theory for this cases, the principle of equating the forces obtained by Blade Element theory with the ones coming from the the changing of momentum in the stream tube is still valid.

\subsection{Large Sectional Rotation BEM (LSR-BEM)}

In this section we will introduce the new mathematical formulation of the BEM theory to take into account the large sectional rotations of blade elements out of the rotor's plane. We are going to start by defining the use of the linear operators that perform the rotation of the physical magnitudes involved (velocities, forces, etc.), consistent with a set of orthogonal matrices of the following form: 


$$
\begin{aligned}
& \mathbf{R}_{\mathbf{x}(\theta)}=\left[\begin{array}{ccc}
1 & 0 & 0 \\
0 & \cos (\theta) & -\sin (\theta) \\
0 & \sin (\theta) & \cos (\theta)
\end{array}\right] \\
& \mathbf{R}_{\mathbf{y}(\theta)}=\left[\begin{array}{ccc}
\cos (\theta) & 0 & \sin (\theta) \\
0 & 1 & 0 \\
-\sin (\theta) & 0 & \cos (\theta)
\end{array}\right] . \\
& \mathbf{R}_{\mathbf{z}(\theta)}=\left[\begin{array}{ccc}
\cos (\theta) & -\sin (\theta) & 0 \\
\sin (\theta) & \cos (\theta) & 0 \\
0 & 0 & 1
\end{array}\right]
\end{aligned}
$$

These matrices perform rotations around the $x, y$, and $z$ coordinate axis. More complex rotations can be obtained by combining these basic matrices, the procedure implies matrix dot product where the order of operations is crucial for the resulting rotation. For example, the product $\mathbf{R}_{\mathbf{z y x}}=\mathbf{R}_{\mathbf{z}(\gamma)} \mathbf{R}_{\mathbf{y}(\beta)} \mathbf{R}_{\mathbf{x}(\alpha)}$ will produce an $\mathbf{R}_{\mathbf{z y x}}$ matrix. The later pre-multiplication of any vector $\mathbf{v}$ will give a $\mathbf{v}^{\prime}=\mathbf{R}_{\mathbf{z y x}} \mathbf{v}$ which is a result of rotating the original $\mathbf{v}$ vector $\alpha$ radians in the $x$ axis, then $\beta$ in the $y$ axis, and finally $\gamma$ radians in the $z$ axis. Note that the transpose of the $\mathbf{R}_{\mathbf{z y x}}$ matrix will not rotate the vector $\mathbf{v}^{\prime}$ back to the original coordinate system. In order to do that, a new matrix $\mathbf{R}_{\mathrm{xyz}}$ has to be obtained from the correct combination of basic rotations. More details on matrix rotations are given in [33-35], among others.

For any rotation matrix $\mathbf{R}_{\mathbf{x}, \theta} \in \mathbb{R}^{3}$ where $x$ is a rotation axis and $\theta$ a rotation angle, the following properties can be enumerated [33,34]:

- $\mathbf{R}_{\mathbf{x}, \theta}^{T}=\mathbf{R}_{\mathbf{x}, \theta}^{-1}$

- $\operatorname{det}\left(\mathbf{R}_{\mathbf{x}, \theta}\right)=1$

- $\mathbf{R}_{\mathbf{x},(\theta+\mathbf{r})}=\mathbf{R}_{\mathbf{x}, \theta} \cdot \mathbf{R}_{\mathbf{x}, \mathbf{r}}$

- $\mathbf{R}_{\mathbf{x}, \mathbf{0}}=\mathbf{I}$ (where $\mathbf{I} \in \mathbb{R}^{n}$ is the identity matrix).

- The eigenvalues of $\mathbf{R}_{\mathbf{x}, \theta}$ are: $\left\{1, e^{ \pm i \theta}\right\}=\{1, \cos (\theta)+i \sin (\theta), \cos (\theta)-i \sin (\theta)\}$.

Now, we write the wind velocity vector Uh facing the differential annulus of our actuator disk, affecting its components, according to BEM theory, by the axial induction factor $a$ and the tangential induction factor $a^{\prime}$. The $h$ subscript here indicates that the wind velocity vector is described in the $h u b$ coordinate system (see figure 3.5) according to standards from the International Electrotechnical Commission (IEC) [36]. 


$$
\mathbf{U h}=\left[\begin{array}{l}
U w h_{1}(1-a) \\
U w h_{2}+\Omega r_{h}\left(1+a^{\prime}\right) \\
U w h_{3}
\end{array}\right],
$$

where $a$ and $a^{\prime}$ are the axial and tangential induction factors respectively, $U w h$ is the incoming wind velocity projected into the $h$ coordinate system, $\Omega$ is the angular velocity of the rotor and $r_{h}$ is the radial distance of the airfoil section in the $h$ coordinate system.

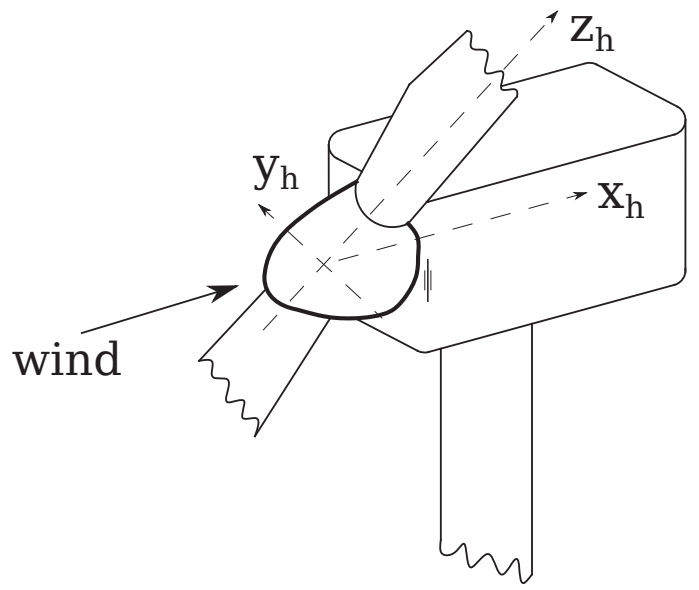

Figure 3.5. Hub coordinate system according to standards from the International Electrotechnical Commission (IEC) [36]

The next step is to compute the relative velocity affecting the blade element. For this, we will project Uh going through the different coordinate systems, from the hub, until reaching the blade's section coordinate system. Let's see first which rotations we shall go through, and which matrices will transform our velocity vector from one coordinate system to the other.

The conning rotation matrix is a linear operator with a basic rotation in the $y$ axis of the $h u b$ coordinate system, $y_{h}$ (see figure 3.5 ), where $\theta_{c n}$ is the conning angle for the rotor (see figure 3.6).

$$
\mathbf{C}_{\theta_{\mathbf{c n}}}=\left[\begin{array}{ccc}
\cos \left(\theta_{c n}\right) & 0 & \sin \left(\theta_{c n}\right) \\
0 & 1 & 0 \\
-\sin \left(\theta_{c n}\right) & 0 & \cos \left(\theta_{c n}\right)
\end{array}\right]
$$

After orienting our wind velocity for conning effects on the rotor, we will orient it for any existing pitch angle defined for the blade. This pitch angle $\theta_{p}$ is the result of adding a pre-set pitch angle $\theta_{p_{0}}$ that may be defined a priori plus an instantaneous pitch-control angle 


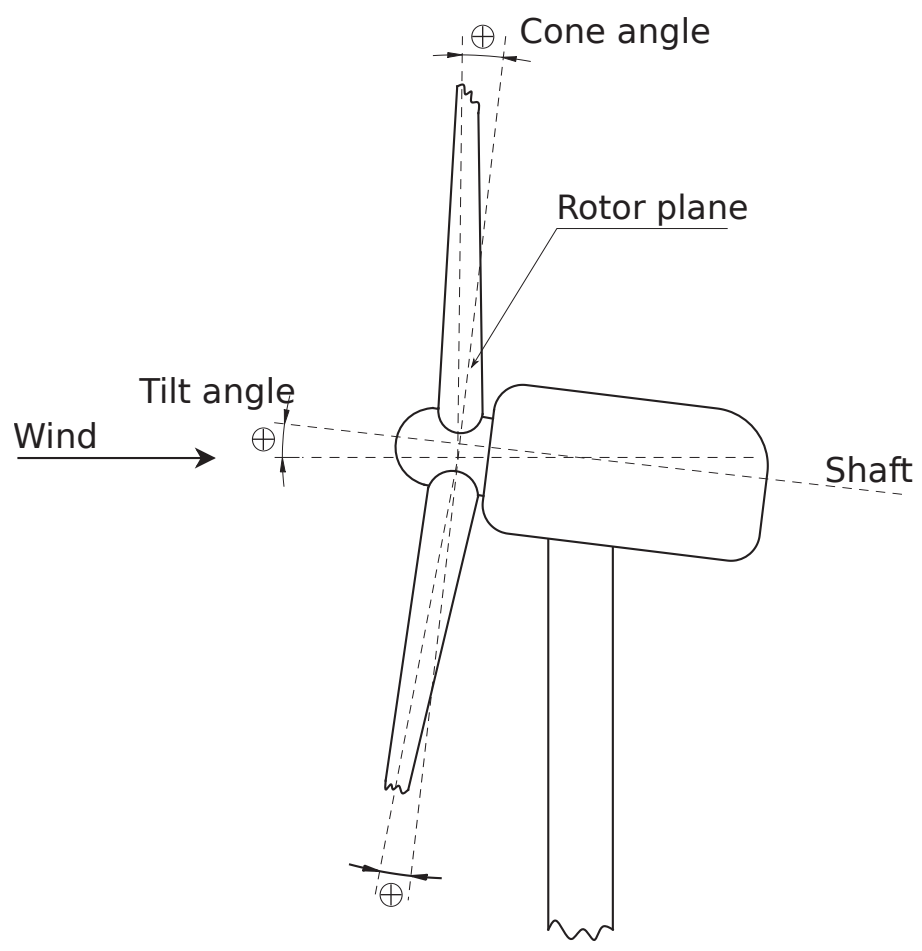

Figure 3.6. Cone and tilt angles for upwind wind turbines, according to standards from the International Electrotechnical Commission (IEC) [36]

$\theta_{p_{c}}$ coming from external control actions. The Pitching rotation matrix $\mathbf{C}_{\theta_{\mathbf{p}}}$ is defined by a rotation around the $z$ axis resulting from the previous conning rotation in the blade's system of coordinates (see figure 3.7 ) referred to by the $b$ subscript,

$$
\mathbf{C}_{\theta_{\mathbf{p}}}=\left[\begin{array}{ccc}
\cos \left(\theta_{p}\right) & -\sin \left(\theta_{p}\right) & 0 \\
\sin \left(\theta_{p}\right) & \cos \left(\theta_{p}\right) & 0 \\
0 & 0 & 1
\end{array}\right],
$$

where $\theta_{p}=\theta_{p_{0}}+\theta_{p_{c}}$. Thus, any transformation from $h$ to $b$ would be given by: $\mathbf{C}_{\mathbf{b h}}=$ $\mathrm{C}_{\theta_{\mathbf{p}}} \mathbf{C}_{\theta_{\mathbf{c n}}}$.

Two more re-orientations are needed in order to get to the instantaneous system of coordinates of the blade sections, defined as $r$ in section 4.1. The first of these matrices contains information on blade section geometry at the time the blade was designed and manufactured. As mentioned previously, the blade could have pre-conformed curvatures along its longitudinal axis (i.e. the blade axis is no longer rectilinear). This curvatures can reflect either an initial twist along the longitudinal axis or a combination of twist plus pre-bending on the other two axes (i.e. conning/sweeping). To this end, we compute during 




Figure 3.7. Blade coordinate system according to standards from the International Electrotechnical Commission (IEC) [36]

the blade design stage a set of transformation matrices which contain the information of the three dimensional orientation of the blade's sections for each position on the longitudinal axis as we move along the span. To this end, we compute the Frenet-Serret formulas that define the curvature of the (now curvilinear) longitudinal axis [37]. These differential formulas provide the means to describe the tangent, normal, and binormal unit vectors on a given curve. Due to these unit vectors, the Frenet-Serret coordinate system is also known as the TNB frame. More information about the calculation of the TNB unit vectors, their properties and other applications can be found in [37,38]. Around the tangential axis of the TNB, there is a further rotation of each blade section to orient it according to the particular twist specified on the blade's aerodynamic design. Combining these rotations we then create a transformation matrix for every blade section at different span positions. We call this matrix the $\mathbf{C}_{\mathbf{R b}}$, as it relates the global coordinate system of the blade $b$, with the system of coordinates of the blade sections in the original configuration $\mathrm{R}$, as defined in section 4.1.

After applying the $\mathbf{C}_{\mathbf{R b}}$, one more projection is needed to get to the instantaneous coordinate system $r$. This last transformation is given by the $\mathbf{C}_{\mathbf{r R}}$ matrix, computed by the $1 \mathrm{D}$ structural model, as it will be explained in section 4.2. It contains information to transform 
vectors from the $R$ to the $r$ systems after structural deformations has occurred. Note that this matrix is updated at every timestep of the 1D model during dynamic simulations, being one of the key variables transporting information between the structural and aerodynamic models.

After all these projections of the Uh vector, we have the relative wind velocity expressed in the blade's section coordinate system, $r$. The expression for $\mathbf{U}_{\text {rel }}$ is as follows:

$$
\mathbf{U}_{\text {rel }}=\left(\mathbf{C}_{\mathbf{r R}} \mathbf{C}_{\mathbf{R b}} \mathbf{C}_{\theta_{\mathbf{p}}} \mathbf{C}_{\theta_{\mathbf{c n}}} \mathbf{U h}\right)+\mathbf{v}_{\mathbf{s t r}}
$$

where the addition of $\mathbf{v}_{\text {str }}$ corresponds to the blade section structural deformation velocities, coming from the structural model. We will return with a detailed definition of this structural deformation velocities later on under section 3.3.3.

Then, the magnitude $\mathbf{U}_{\text {rel }}$ and the angle of attack $\alpha$ are used to compute the forces on the airfoil section through the aerodynamic coefficients $\mathrm{Cl}, \mathrm{Cd}$.

$$
d F_{l}=\frac{1}{2} \rho C_{l}\left|U_{r e l}\right|^{2} c, \quad d F_{d}=\frac{1}{2} \rho C_{d}\left|U_{r e l}\right|^{2} c
$$

Another innovation of our model is that the data tables from static wind-tunnel are corrected at each timestep to consider either rotational-augmentation or dynamic-stall effects, or both. A detailed description of our implementation of the rotational-augmentation and the dynamic-stall corrections will be covered in 3.3.

The aerodynamic loads acting on the blade element

$$
\mathbf{d F r}=\left[\begin{array}{c}
0 \\
d F_{d} \\
d F_{l}
\end{array}\right]
$$

are then projected back onto the $h$ coordinate system through

$$
\mathbf{d F h}=\mathbf{C}_{\theta_{\mathbf{c n}}}{ }^{T} \mathbf{C}_{\theta_{\mathbf{p}}}{ }^{T} \mathbf{C}_{\mathbf{R b}}{ }^{T} \mathbf{C}_{\mathbf{r R}}{ }^{T} \mathbf{C}_{\mathbf{L t h a l}} \mathbf{d F r},
$$

where $\mathbf{C}_{\mathbf{L t h a l}}$ is the matrix which projects the lift and drag forces onto the chord-wise and chord-normal directions, which are aligned with the coordinates of the $r$ system,

$$
\mathbf{C}_{\text {Lthal }}=\left[\begin{array}{ccc}
1 & 0 & 0 \\
0 & -\cos (\alpha) & \sin (\alpha) \\
0 & \sin (\alpha) & \cos (\alpha)
\end{array}\right]
$$


Finally, as in the classic BEM theory, the force

$$
\mathbf{d F h}=\left[\begin{array}{c}
d F_{N} \\
d F_{T} \\
0
\end{array}\right]
$$

is equated to the rate of change of momentum in the annular streamtube corresponding to the blade element. The component normal to the rotor's disk, $d F_{N}$, is equated to the change in axial momentum, while the tangential component, $d F_{T}$, is equated to the change of angular momentum. Thus, for the axial component we have:

$$
B d F_{N}=4 \pi \rho|\mathbf{U h}|^{2} a(1-a) r
$$

Burton et al. [27] propose an extra term that takes into account the fact that the rotation of the wake causes a drop in the wake pressure equal to the increase of a dynamic head

$$
\frac{1}{2} \rho\left(2 a^{\prime} \Omega r\right)^{2}
$$

Therefore, there is an additional axial force on the annulus, equal to:

$$
2 \pi r \frac{1}{2} \rho\left(2 a^{\prime} \Omega r\right)^{2}
$$

Taking 3.31 into account, equation3.29 can be expanded to:

$$
B d F_{N}=4 \pi \rho F_{f}\left(|\mathbf{U h}|^{2} a(1-a)+\left(2 a^{\prime} \Omega r\right)^{2}\right) r
$$

where $F_{f}$ is the combination of the tip and hub loss factors, both described in section 3.1, equations $3.9,3.10$. This nonlinear expresion in $a$ is going to be solved by an advanced iterative algorithm which will be described in detail in the following section.

Equating now the axial rotor torque, caused by the tangential aerodynamic forces on the rotor, with the rate of change of angular momentum of the air passing through the swept annulus, we obtain from BEM theory the expression:

$$
B d F_{T}=4 \pi \rho F_{f}|\mathbf{U h}|(\Omega r) a^{\prime}(1-a) r^{2},
$$

which gives the explicit expression for the tangential induction factor: 


$$
a^{\prime}=\frac{-B d F_{T}}{4 \pi \rho F_{f}|\mathbf{U h}| \Omega(1-a) r^{2}} .
$$

\subsubsection{Iterative solution of the induction factors}

For the solution of the induction factors (i.e. the interference model) at each timestep of the aeroelastic solution, we apply an iterative method. We solve for the axial induction factor $a$, minimizing the residual of equation 3.32. Equation 3.34 is updated during the iteration process that solves for $a$. For the minimization of the residual we use the fzero function from Matlab. fzero uses a combination of bisection, secant, and inverse quadratic interpolation methods to find the root of a continuous function of one variable. The main advantage of applying this close-loop method is that the convergence criteria, as well as the error check, is constantly monitored by Matlab using efficient, proven, and highly reliable numerical algorithms $[39,40]$.

Once the interference factors are converged, a final correction should be made to the axial induction factor $a$ when the incoming wind flow is not perfectly perpendicular to the actuator disk. Corrections to the skewed wake come from helicopter rotor theory, among those is the Method of Acceleration Potential developed by Prandtl. For the case of axisymmetric pressure distributions on the rotor disk, Pitt and Petters $[29,30]$ arrived at an expression for the correction of the axial induction factor using the yaw angle of the rotor instead of the wake skew angle. Applying this correction, our new axial induction factor is:

$$
a=a_{0}\left(1+\frac{15 \pi}{32} r \tan \left(\frac{\psi}{2}\right) \sin (\Omega t)\right),
$$

where $a_{0}$ is the previously converged axial induction factor, $\psi$ is the yaw angle of the rotor and $\Omega t$ is the instantaneous angular velocity of the rotor. Once the iterative algorithm for the interference model achieves convergence, we compute the aerodynamic forces acting on each blade section following part of the process from equations 3.20 to 3.28 , plus the pitching moment on the airfoil section computed from the classic pitch coefficient $\mathrm{Cm}$.

\subsubsection{Interference model for the "turbulent-wake" state}

Classic BEM theory defines the thrust coefficient as as $C T=4 a(1-a)$. This quadratic expression returns a maximum $C T$ when the axial induction factor is $a=0.5$ [26]. Beyond this point the interference model for the rotor disk leaves what in the wind-power jargon is called the "windmill state" and enters the so called "turbulent-wake state". In section 3.1, basic assumptions of momentum theory become invalid at this point and, in order to take 


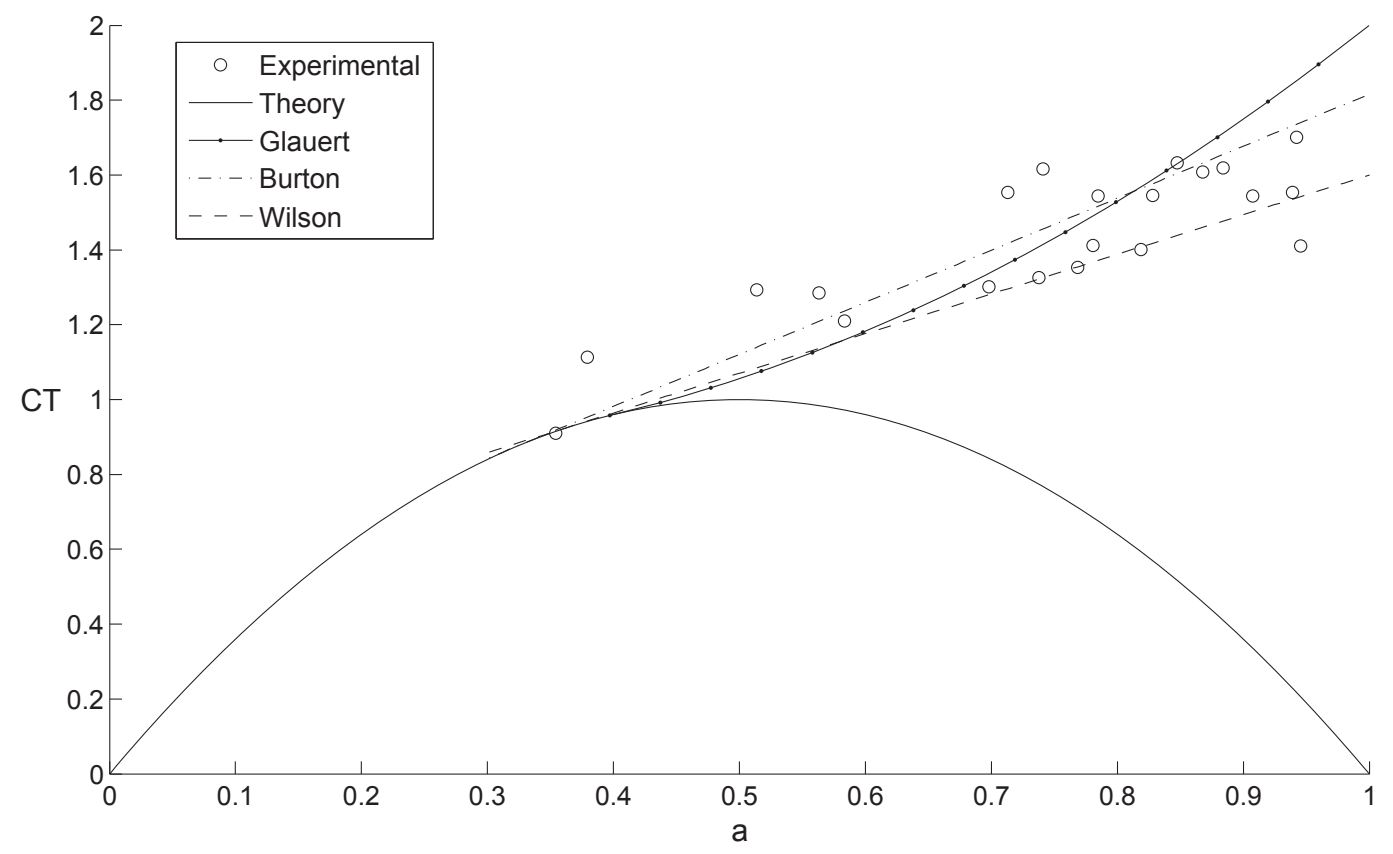

Figure 3.8. Empirical relations fitting Lock experimental data [41] for wind turbines operating in the turbulent wake state.

into account part of the flow in the far wake which starts to propagate upstream, a correction to the theory is needed. Physically, this flow reversal cannot occur, and what actually happens is more flow entrains from outside the wake and the turbulence increases. The flow behind the rotor slows down but the thrust continues to increase $[26,27]$.

Glauert was the first to propose an empirical relation to overcome this limitation in BEM theory [28]. He fitted a parabolic function to the experimental data from Lock et al. for wind turbines operating in the turbulent wake state [41]. Glauert's fitting function is tangent to the original $C T$ curve at $a=0.4$. Other authors such as Burton [27] and Wilson [42] proposed after Glauert different fitting functions to the experimental data as we can see in figure 3.8. Nevertheless, a discontinuity between the fitting function and the original $C T$ function appears when correction factors for tip and hub losses are taken into account in BEM theory [43]. This discontinuity is critical when the induction factors are to be obtained implementing numerical approaches for BEM theory. Marshall Buhl, Jr. proposes a new empirical relationship for the thrust coefficient and the axial induction factor for the turbulent wake state. His relation not only takes into account for the tip and hub losses correction factors but also solves the gap problem existing in other previous works [43].

Although Buhl's empirical relation solves the gap problem, it doesn't seem to minimize the error for the existing experimental data. To overcome this problem, we propose in this 
work a new empirical relation based on fitting a power-law function to Lock's experiments:

$$
\Psi(a)=1.724(a-0.280)^{0.225}
$$

This power-law function seems to be a more natural match to the original $C T$ function as it starts departing from the theoretical parabolic shape for induction factors greater than $a=0.3$, as observed by Lock in his experiments. Figure 3.9 shows a comparison between Glauert's, Buhl's, and our proposed power-law fitting function for a $C T$ curve affected by a loss factor $F_{t}=0.9$. We can see here the gap mentioned before and how Buhl's empirical relation overcomes this problem [43]. In the same way, the nature of our power-law function automatically solves the gap problem as it cuts the original $C T$ function instead of being just tangent in one point. For our interference model, the decision of whether to compute the thrust coefficient $C T$ using the theoretical or the power-law function is automatically made by simply solving the intersection of both curves and deciding the region of the actual induction factor computation. With this we gain flexibility compared to the fixed limit imposed in other methods to consider theoretical or empirical calculations. Table 3.1 shows the Mean Square Error (MSE) of the different empirical relations fitting Lock's experimental data. These results confirm that, besides the advantages previously mentioned, the proposed power-law function produces an error about one order of magnitude smaller that the other options.

Table 3.1

MSE of the different empirical approximations to Lock's experimental data.

\begin{tabular}{lc}
\hline Empirical relation & $M S E=\frac{1}{n} \sum_{i=1}^{n}\left(x_{i}-\overline{x_{i}}\right)^{2}$ \\
\hline Glauert & 0.0291 \\
Buhl & 0.0291 \\
Wilson & 0.0201 \\
Burton & 0.0166 \\
Power-law & 0.0094 \\
\hline
\end{tabular}

Thus, if during computations of our interference model the axial induction factor is equal or greater to the value where the theoretical $C T$ function intersects the power-law fitting function, instead of minimizing the residual of Eq. 3.32, we minimize the residual of:

$$
0=\Psi(a)-C T_{l}
$$

where the local thrust coefficient, $C T_{l}$ is: 


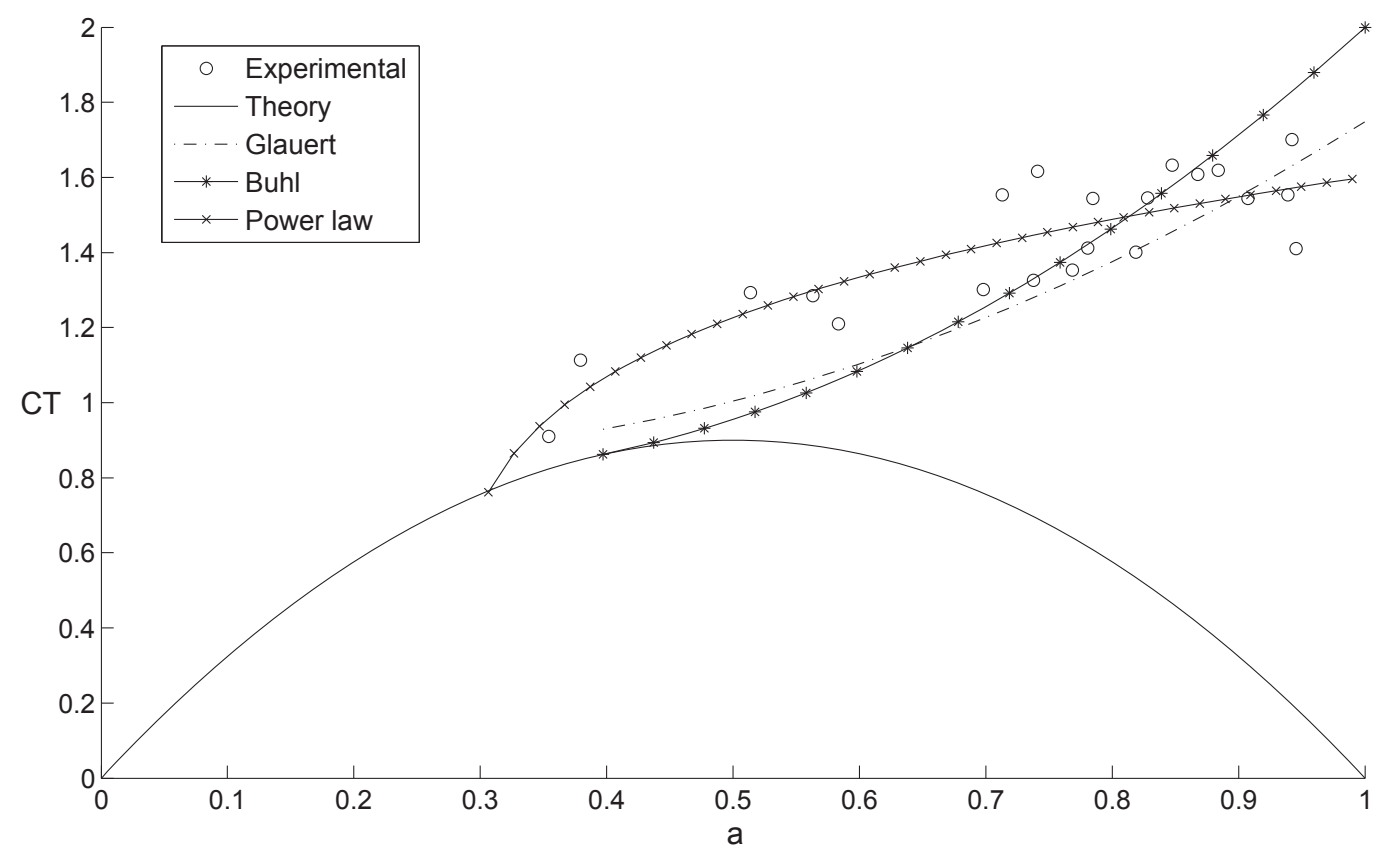

Figure 3.9. Power Law, Buhl's, and Glauert's empirical relations fitting the Lock experimental data [41]. Theoretical $C T$ curve affected by a loss factor $F_{t}=0.9$.

$$
C T_{l}=\frac{B d F_{N}}{\rho\left|U_{(h)}\right|^{2} \pi r}
$$

\subsection{The Flow Model Interface (FMI)}

As described in section 2.2, the FMI is the module which computes the aerodynamic forces on the blade sections that are going to be used later in the structural model. In this section we cover a series of operations that are needed prior to solving the LSR-BEM interference model. These operations include coefficients, and the computation of the incoming wind vector taking into account the displacement of the blade section given by the solution of the structural model for the previous timestep.

\subsubsection{Pre-processing of the airfoil aerodynamic coefficients}

Airfoil aerodynamic coefficients under FMI are obtained from static airfoil data coming from wind tunnel tests as in Aerodyn [10,11], nevertheless data processing in the FMI was conceived to overcome several limitations of Aerodyn, as described under [24]. Aerodyn 
presents two instances where the process of static airfoil data is required: The first one makes a preprocessing of the data on an external program, AirfoilPrep $[11,44]$, correcting the aerodynamic coefficients to take into account three-dimensional effects such as rotational augmentation $[45,46]$, which is performed before the aeroelastic solution starts and it is not updated during the time-integration process. The second one is the implementation of a dynamic stall model [47] which, even though it is part of Aerodyn itself, it is applied only to the final computation of the aerodynamic loads but not coupled with the iterative process of the interference BEM model. In our FMI however, this treatment of the coefficients is fully integrated into the time marching problem correcting and updating the airfoil coefficient tables to account for the aerodynamic conditions that the rotor goes through during the simulation. As we will explain in the following paragraphs, this conception of integrating the aerodynamic coefficients processing into the aeroelastic "package" is twofold: First, to obtain accurate coefficients corrections according to the simulation circumstances, and second, to allow for the use of multiple airfoil aerodynamic data tables to use on demand and in real-time, covering a wide range of aerodynamic conditions if experimental data is available.

\section{Multiple airfoil aerodynamic data tables}

One of the possibilities the FMI allows is to add multiple data tables for the different airfoils and use them according to the instantaneous aerodynamic situations on the rotor. One implemented possibility is to have multiple Reynolds data tables and use them according to the instantaneous Reynolds number for the blade section. Moreover, interpolations between data tables is also possible when the computed instantaneous Reynolds number lays between two sets of data. Another possibility would be to include aerodynamic coefficients from modified airfoils with active control surfaces as micro-tabs or deployable spoilers. In these cases, an external control module would determine which data table to use for each blade section according to a pre-defined control strategy.

\section{Rotational Augmentation}

The FMI processes the aerodynamic coefficient tables and provides the needed updated variables to the stall-delay model from Du \& Selig [45] to incorporate rotational augmentation effects to the lift coefficients in real-time. Likewise, corrections for the drag coefficients according to Eggers' model [46] are computed at every timestep of the simulation. This feature of the FMI overcomes a limitation of its predecessor, Aerodyn, as discussed in [24].

Du \& Selig's model modifies the 2D airfoil data to simulate 3D stall-delay effects on 
rotor airfoils. From the analysis of the 3D integral boundary-layer equations for a reference system rotating with the blade, and the rotational effects at its separation point, Du \& Selig determined a semi-empirical correction formula to incorporate the effects of a delayed boundary layer separation on rotating airfoils [45]. This means that the stall angle of attack resulting from static wind tunnel tests would be larger and its corresponding lift coefficient $C_{l}$ would be greater. Thus, a $\Delta C_{l}$ should be added to the original 2D data:

$$
\Delta C_{l}=f_{l}\left(C_{l, p}-C_{l(2 D)}\right),
$$

where $C_{l, p}$ is a modified lift coefficient depending on the angle of zero lift for the airfoil, and $f_{l}$ is a semi-empirical correction function of the form:

$$
f_{l}=\frac{1}{2 \pi}\left(\frac{1.6(c / r)}{0.1267} \frac{a-(c / r)^{\frac{d}{\Lambda} \frac{R}{r}}}{b+(c / r)^{\frac{d}{\Lambda} \frac{R}{r}}}-1\right),
$$

where $a, b, d$ are empirical constants, $c / r$ is the airfoil chord to local radial position ratio, $R$ is the rotor radius, and $\Lambda$ is a modified tip speed ratio. More details on this model can be found in [45]. As defined for Aerodyn in [44], we also correct the drag coefficients for rotational augmentation effects using Eggers' model [46]. From his work, a $\Delta C_{d}$ is added to the 2D drag coefficient $C_{d}$. According to the implementation in [44], this $\Delta C_{d}$ uses the previously computed $\Delta C_{l}$ from Du \& Selig as:

$$
\Delta C_{d}=\Delta C_{l}\left(\frac{\sin (\alpha)-0.12 \cos (\alpha)}{\cos (\alpha)+0.12 \sin (\alpha)}\right) .
$$

\section{Viterna Extrapolation}

After correcting the aerodynamic coefficients, the FMI applies the Viterna's extrapolation method [48] to ensure the data availability for a range of angles of attack $\pm 180^{\circ}$. The implementation of Viterna's method is the same as in AirfoilPrep [11], the only significant difference is that in FMI it is also computed in real-time, similar to the rotational effects previously described.

Viterna proposes the extrapolation of the lift and drag coefficients assuming that the airfoil will behave as a flat plate for high values of the angle of attack [48]. With this, a maximum drag coefficient $C_{d_{\max }}$ is computed depending on the aspect ratio $A R$ of the flat plate as: 


$$
C_{d_{\max }}=1.11+0.018 \mathrm{AR} .
$$

With $C_{d_{\max }}$, and knowing the last available angle of attack among the data, an extrapolation of the data up to $\alpha=90^{\circ}$ is done by applying a combination of trigonometric functions:

$$
\begin{gathered}
C_{d}=C_{d_{\max }} \sin ^{2}\left(\alpha+\zeta_{1} \cos (\alpha)\right) \\
C_{l}=\frac{1}{2} C_{d_{\max }} \sin (2 \alpha)+\zeta_{2} \frac{\cos ^{2}(\alpha)}{\sin (\alpha)},
\end{gathered}
$$

where the expressions for $\zeta_{1}$ and $\zeta_{2}$ are:

$$
\begin{gathered}
\zeta_{1}=\frac{C_{d_{s}}-C_{d_{\max }} \sin ^{2}\left(\alpha_{s}\right)}{\cos \left(\alpha_{s}\right)} \\
\zeta_{2}=\frac{\sin \left(\alpha_{s}\right)}{\cos ^{2}\left(\alpha_{s}\right)}\left(C_{d_{s}}-C_{d_{\max }} \sin \left(\alpha_{s}\right) \cos \left(\alpha_{s}\right)\right) .
\end{gathered}
$$

These extrapolations yield to $C_{l}=0$ and $C_{d}=C_{d_{\max }}$ at $\alpha=90^{\circ}$. From $90^{\circ}<\alpha<$ $180^{\circ}$ the values are mirrored from the extrapolated coefficients to complete the half span. For the negative range of $\alpha$, data is reflected from the positive range. Lift coefficients $C_{l}$ are reduced by a factor of 0.7 to account for airfoil asymmetry, as explained in [11]. Pitching moment coefficients are also extrapolated during the same process. Although this technique is not included in Viterna's work, the extrapolation of the pitching moment coefficients is done according to the references in [11]. Figure 3.10 shows extrapolated airfoil coefficients for two airfoils, a thick airfoil from the DU family, the DU 91-W-250, and a thin airfoil NACA 64-618.

\section{Dynamic stall}

Horizontal and vertical wind shears, vertical wind components, and general turbulence in the wind alter its velocity over the rotor. These variations produce an oscillatory time history of the angle of attack on the wind turbine blade airfoil sections [10]. As in Aerodyn [10,11], the FMI allows for the possibility to include dynamic-stall effects on the blade sections. As an improvement compared to Aerodyn, we implemented the possibility to compute these effects either during the iterative calculation of the induction factors inside 


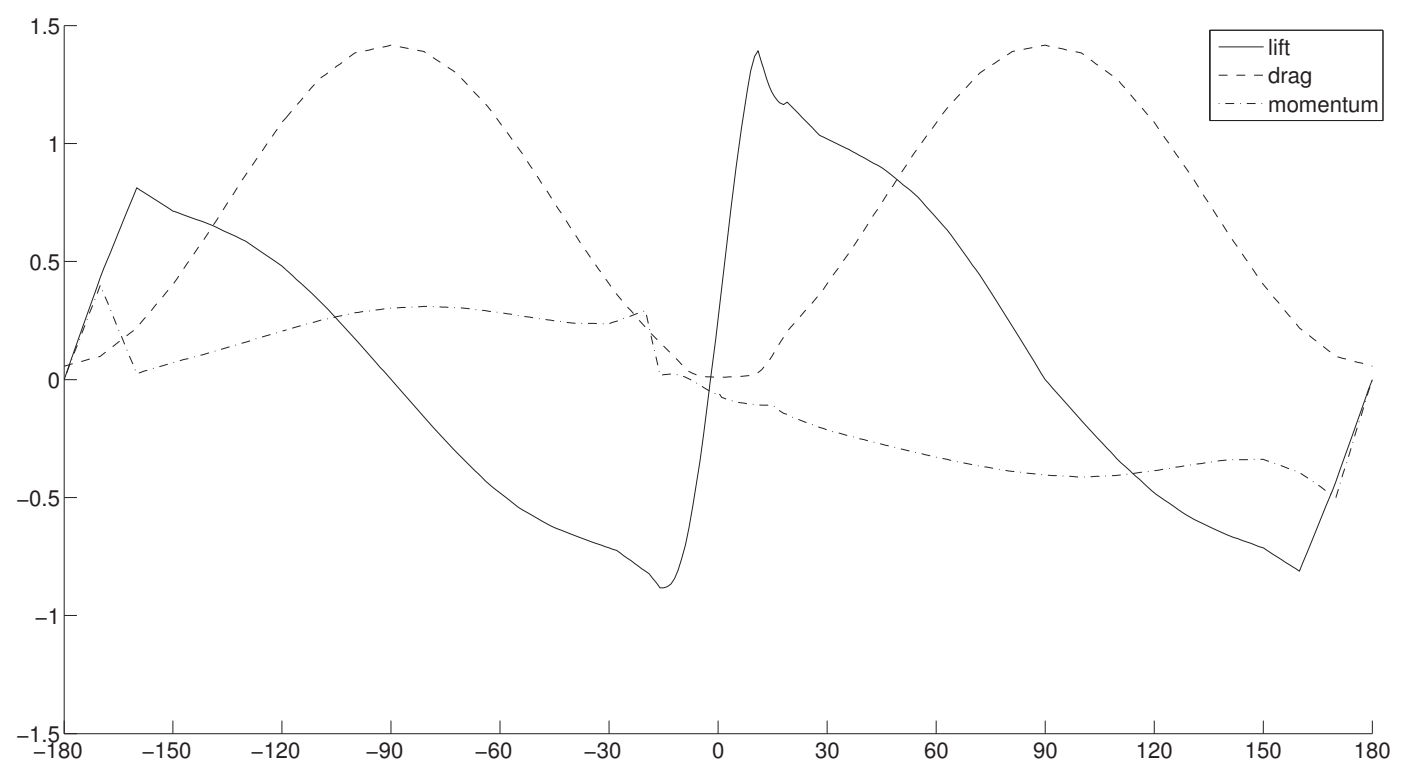

(a)



(b)

Figure 3.10. Viterna's extrapolation of airfoil aerodynamic coefficients for: (a) a DU 91-W-250 airfoil, and (b) a NACA 64-618 airfoil.

the LSR-BEM model or once the induction factors are converged, as discussed in [24]. The dynamic stall model implemented on our FMI is, as in Aerodyn, based in the work of Beddoes and Leishman [47,49,50], using also as fundamental guides for the implementation, the two Master of Science theses from the University of Utah by Pierce [51] and Minnema [52]. In this work we will briefly introduce the concepts of this theory, neverthe- 
less, for a thorough detailed explanation, we recommend the reader to consult the above mentioned references.

The semiempirical dynamic-stall model from Beddoes-Leishman could be divided into three main stages: 1) The computation of the attached flow response in the airfoil section. 2) The modification of that attached flow response based on the position of the effective flow separation point on the low-pressure side of the airfoil [10]. 3) A vortex model is applied to account for shedding effects that occur during dynamic stall.

The Beddoes-Leishman model is based on airfoil indicial response [10,51]. This indicial response is derived from the linearized differential equations for an unsteady, compressible, inviscid fluid solution from the work of Bisplinghoff et al. [53]. Two main coefficients result from this indicial response, the normal force coefficient $C_{N}$ and the moment coefficient $C_{M}$. Step changes in the airfoil's angle of attack, $\Delta \alpha$, originate time functions of these coefficients. In the case of $C_{N}$, the increment due to $\Delta \alpha$ could be split in two components[10], a non-circulatory, $C^{k}{ }_{N}$, and a circulatory, $C^{c}{ }_{N}$, expressed as:

$$
\begin{gathered}
\Delta C^{k}{ }_{N}=\frac{4}{M} \psi^{k}{ }_{\alpha} \Delta \alpha \\
\Delta C^{c}{ }_{N}=C_{N \alpha} \psi^{c}{ }_{\alpha} \Delta \alpha,
\end{gathered}
$$

where $M$ is the Mach number, $C_{N \alpha}$ is the curve slope of the normal force coefficient, and $\psi^{k}, \psi^{c}$ are the non-circulatory and circulatory indicial functions, being $\psi$ a nearly pure exponential function [10]. Acording to a given time-evolution history of the airfoil's angle of attack, $\alpha$, the attached flow response is computed from the superposition of individual indicial responses for each step. Figure 3.11 shows the hysteresis obtained from measured $C_{M}$ coefficients on a NACA 4415 airfoil for a determined sinusoidal oscillation of the angle of attack, $\alpha$. For this same airfoil, figure 3.12 shows dynamic $C_{N}$ coefficients hysteresis loops for unusual angle of attack ranges computed using the Beddoes-Leishman model [10]. As explained in [10], it is difficult to verify the accuracy of the model for high angles of attack due to the lack of experimental data.

In the modification of that attached flow response, the second stage, aerodynamic coefficients are reduced from the attached flow values due to the flow separation which results in a loss of circulation about the airfoil [10]. Beddoes computes the flow separation point using an approximation to Kirchhoff theory [54] which relates the normal force coefficient, $C_{N}$, and the chordwise force coefficient, $C_{C}$, as:

$$
C_{N}=C_{N \alpha}\left(\alpha-\alpha_{0}\right)\left(\frac{1+\sqrt{f}}{2}\right)^{2}
$$




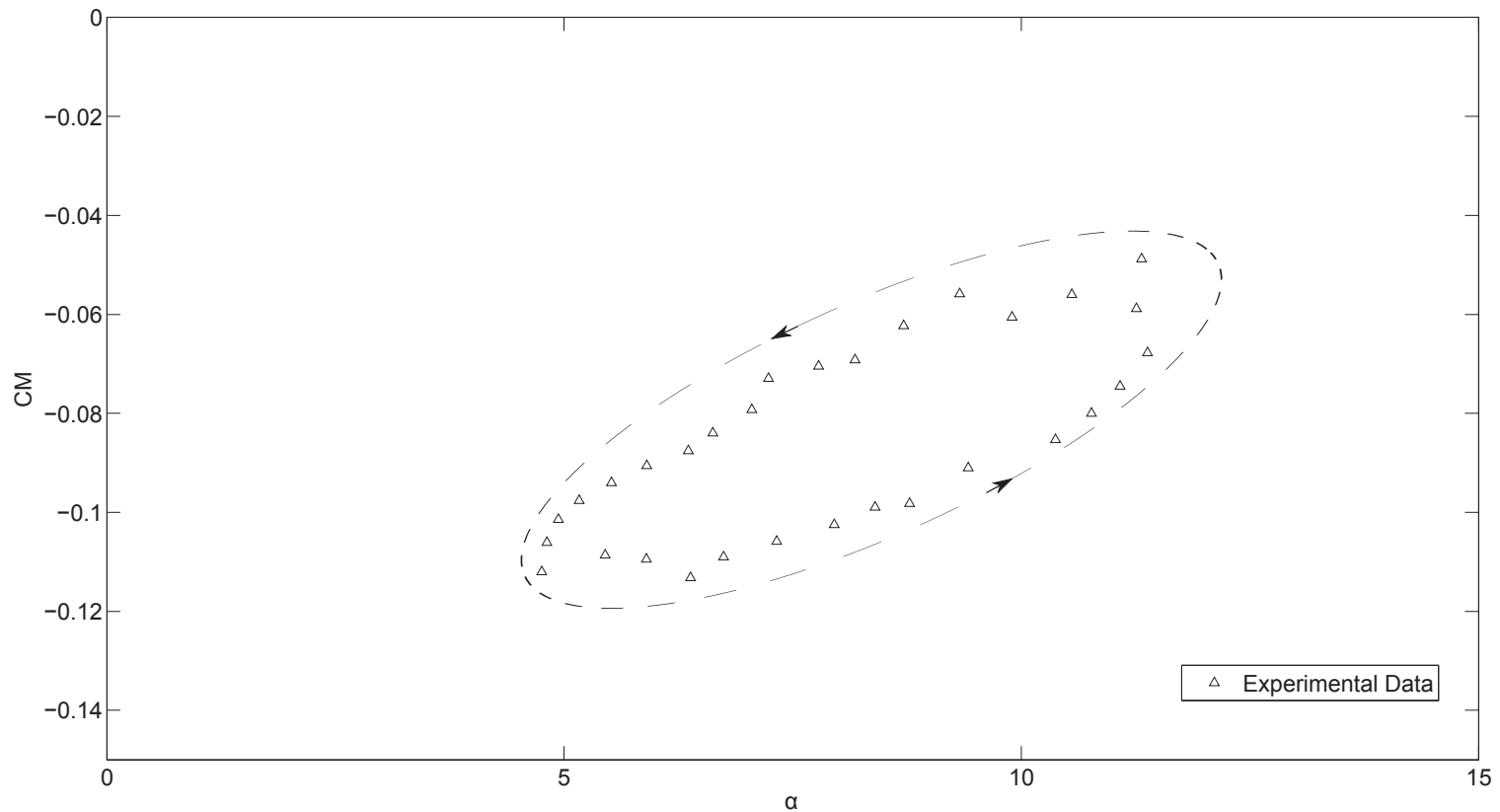

Figure 3.11. Hysteresis loops obtained from measured $C_{M}$ coefficients on a NACA 4415 airfoil under $R e=1 \times 10^{6}$, for a sinusoidal function: $\alpha=8+3.5 \sin (\omega t), \omega_{\text {red }}=0.089, f=1.841 \mathrm{~Hz}$.

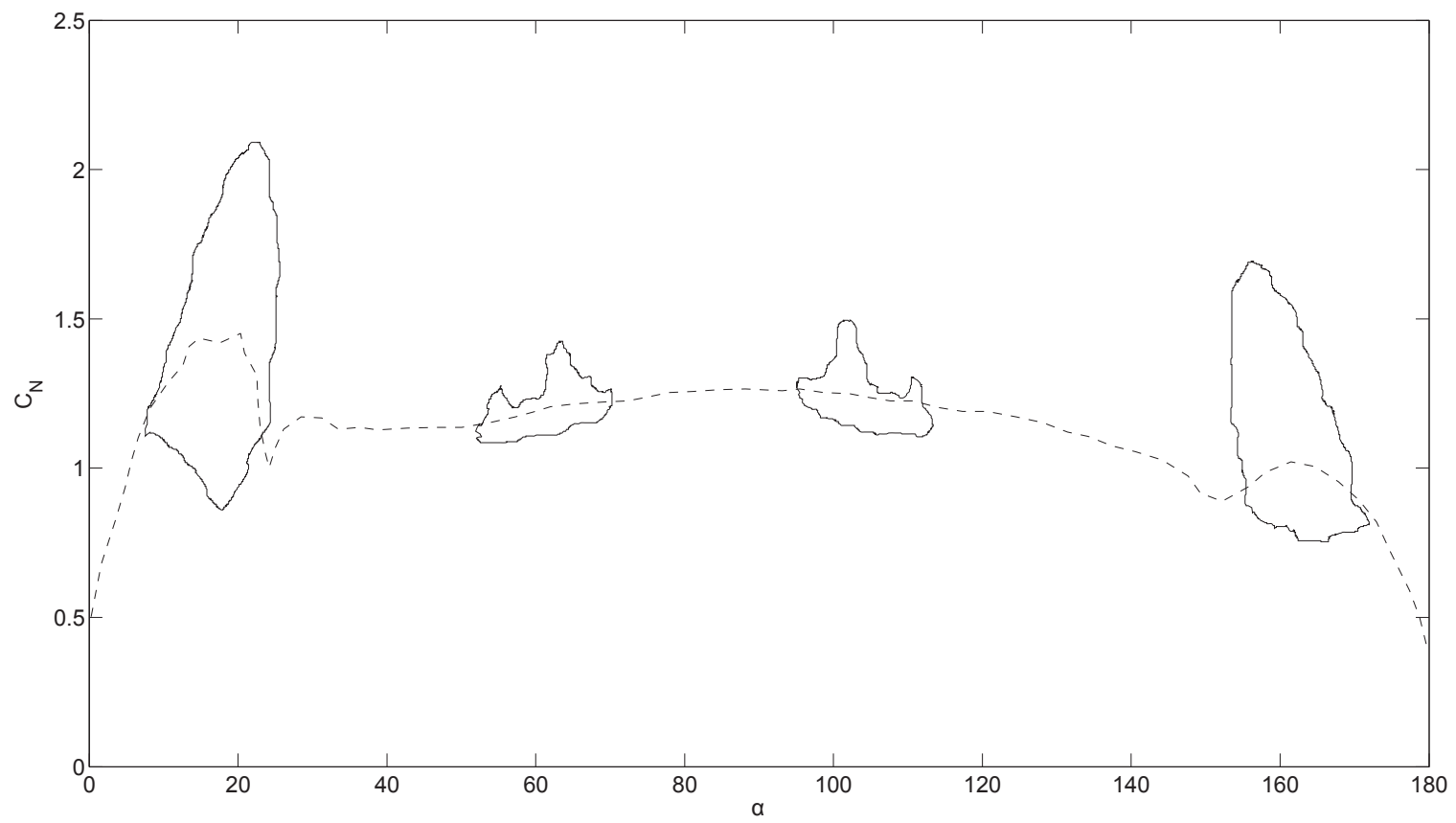

Figure 3.12. $C_{N}$ coefficients hysteresis loops computed for a NACA 4415 airfoil at unusual angles of attack using the Beddoes-Leishman model. 


$$
C_{C}=C_{N \alpha}\left(\alpha-\alpha_{0}\right) \tan (\alpha) \sqrt{f},
$$

where $\alpha_{0}$ is the zero lift angle of attack. From equations 3.49 and 3.50, the effective separation point $f$ is computed. Although this separation point comes from Kirchhoff theory which assumes the flow to be inviscid, the solution is good enough to provide a method of representing the effect and dynamics of the separation phenomena [10].

The final stage in the Beddoes-Leishman model is where the vortex shedding happening during dynamic stall is calculated. An empirical model creates an excess of circulation in the vicinity of the airfoil to account for the vortex lift contribution. This contribution is the difference between the attached flow $C_{N}$ value and the value obtained from equation 3.49. Empirical time constants control the growth, decay, and motion of the vortex. Vortex grows its strength when the airfoil pitches nose-down and it is allowed to convect across the airfoil under certain conditions. In addition, a time constant tracks the position of the vortex decaying its strength exponentially as it reaches the trailing edge [10].

After these stages, lift and drag coefficients for the airfoil are computed by re-projecting $C_{N}$ and $C_{C}$ into normal and parallel components to the wind velocity direction as:

$$
\begin{gathered}
C_{l}=C_{N} \cos (\alpha)+C_{C} \sin (\alpha) \\
C_{d}=C_{N} \sin (\alpha)-C_{C} \cos (\alpha)+C_{D 0},
\end{gathered}
$$

where $C_{D 0}$ is the minimum drag coefficient for the airfoil.

Some modifications to the Beddoes-Leishman model are needed in order to make it suitable for wind turbines. A complete detailed explanation of these modifications can be found in [10,51,52]. Figures 3.13 and 3.14 show benchmarks of moment coefficients $C_{M}$ taken from wind tunnel experiments versus computations with our actual implementation of the dynamic stall model for an S809 airfoil. For both cases, the oscillation of the angle of attack is determined by a sinusoidal function.

\subsubsection{Orthogonal projection matrices associated with the action of nacelle mecha- nisms}

As we described in section 3.2, there are several projection matrices that need to be defined before getting into the computations of the LSR-BEM interference model. During the first stages of the FMI module, data from the basic blade design and the kinematic solution for the 1D model at a previous timestep (or initial condition if applicable) is uploaded. Among this data, the blades pitch angle as well as the rotor cone, tilt, yaw, and instanta-

neous azimuth angles will define respective projection matrices. Projection matrices for 


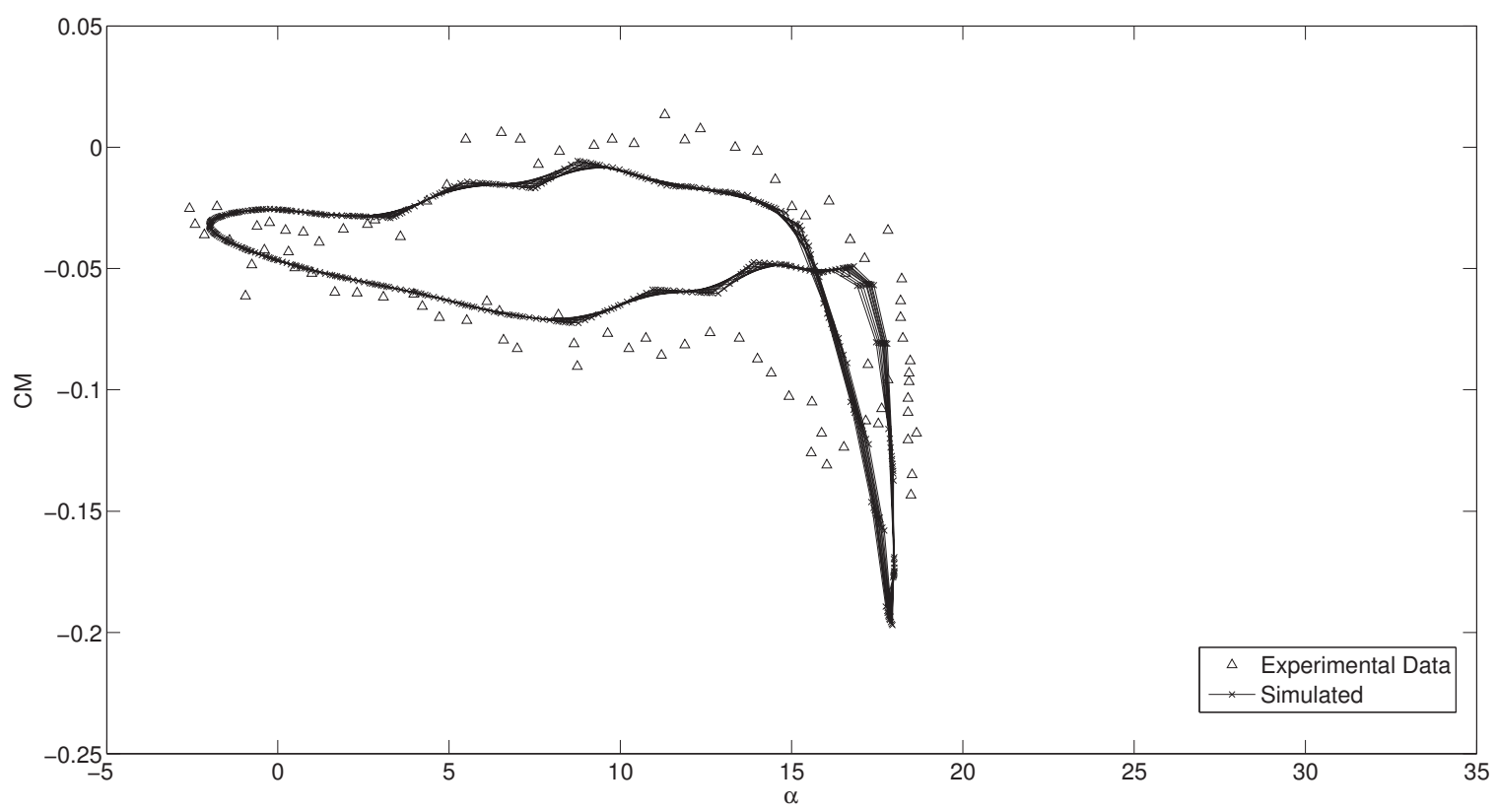

Figure 3.13. Moment coefficient $C_{M}$ benchmark for an $\mathrm{S} 809$ airfoil. Wind tunnel data vs. computations with our actual implementation of the dynamic stall model. $R e=1 \times 10^{6}$, sinusoidal function: $\alpha=8+10 \sin (\omega t), \omega_{\text {red }}=0.026, f=1.82 \mathrm{~Hz}$.



Figure 3.14. Moment coefficient $C_{M}$ benchmark for an $\mathrm{S} 809$ airfoil. Wind tunnel data vs. computations with our actual implementation of the dynamic stall model. $R e=1 \times 10^{6}$, sinusoidal function: $\alpha=14+10 \sin (\omega t), \omega_{\text {red }}=0.080, f=1.85 \mathrm{~Hz}$. 
pitch and cone effects were already described under equations 3.22 and 3.21, tilt effect on the rotor defines another linear operator rotating a $\tau$ angle on the $y$ axis of the hub's coordinate system according to the international standards defined in [36] (see figures 3.6 and 3.5).

$$
\mathbf{C}_{\tau}=\left[\begin{array}{ccc}
\cos (\tau) & 0 & -\sin (\tau) \\
0 & 1 & 0 \\
\sin (\tau) & 0 & \cos (\tau)
\end{array}\right]
$$

As mentioned in section 2.1, the interaction with external control modules require a constant update of some projection matrices. For example, the rotor azimuth matrix, besides the instantaneous position of the blade along its rotation, can reflect control actions on the dynamics of the Electro-Mechanical train that define the rotor's angular speed, $\Omega$. Equation 3.53 shows the linear operator which defines a rotation on the $x$ axis of the hub's coordinate system. Another projection matrix able to reflect control actions is the one for rotor yaw. Equation 3.54 defines this linear operator rotating a $\psi$ angle, as previously defined in equation 3.35, on the $z$ axis of the hub's coordinate system (see [36]).

$$
\begin{gathered}
\mathbf{C}_{\boldsymbol{\Omega}}=\left[\begin{array}{ccc}
1 & 0 & 0 \\
0 & \cos (\Omega t) & \sin (\Omega t) \\
0 & -\sin (\Omega t) & \cos (\Omega t)
\end{array}\right] \\
\mathbf{C}_{\psi}=\left[\begin{array}{ccc}
\cos (\psi) & \sin (\psi) & 0 \\
-\sin (\psi) & \cos (\psi) & 0 \\
0 & 0 & 1
\end{array}\right]
\end{gathered}
$$

Hansen [55], uses the same matrices to re-project the incoming wind vector and then compute aerodynamic forces on a rotor through a classic BEM approach, without taking into account any structural deformation.

\subsubsection{Coupling with the structural model}

As mentioned previously, one of the main advantages of the LSR-BEM is the ability to incorporate all the deformations computed by the structural model at each timestep. In the classical BEM theory, the radial positions of the blade sections are constant, which constitutes an important limitation of Aerodyn, as discussed in [24]. Contrarily, in the LSR-BEM, the positions of the blade sections are not constant anymore, as they actually reflect not only radial displacements but also displacements in any of the two other direc- 
tions. Thus, we will compute the instantaneous position of each blade section in the hub's coordinate system, $h$, using the corresponding projection matrices:

$$
\mathbf{p}_{\mathbf{h}}=\mathbf{C}_{\theta_{\mathbf{c n}}}{ }^{T} \mathbf{C}_{\theta_{\mathbf{p}}}{ }^{T}\left(\mathbf{x y z}_{\mathbf{b}}{ }^{T}+\mathbf{C}_{\mathbf{R b}}{ }^{T} \mathbf{u}^{T}\right)+[0,0, R h u b]^{T},
$$

where $R h u b$ is the hub radius, $\mathbf{x y z}_{\mathbf{b}}$ contains the original position of the blade section in the blade's coordinate system $b$ before deformation (i. e. given by the blade design), and vector $\mathbf{u}=\left[u_{1}, u_{2}, u_{3}\right]$ contains the linear displacements for the blade section coming from the solution of the structural model which represents the instantaneous deformation.

Finally, the position of the blade section in the ground coordinate system, according to the standards from the IEC [36], is given by:

$$
\mathbf{p}_{\mathbf{G}}=\mathbf{C}_{\tau}^{T}\left(\mathbf{C}_{\boldsymbol{\Omega}}^{T} \mathbf{p}_{\mathbf{h}}+[o h, 0,0]^{T}+[0,0, H h u b]^{T}\right)
$$

where oh is the distance defined from the yaw axis to the rotor plane (known as overhang), and $H h u b$ is the height at where the hub is located in the wind turbine from the ground level.

The incorporation of this structural result in the computation of the interference model and the ability to obtain the aerodynamic loads in the deformed blade coordinate system allow us to overcome another main limitation of Aerodyn [10,11]. As discussed in [24], Aerodyn receives from its structural counterpart, FAST [56], the velocity of the blade-section motion due to deformation, and adds it to the incident wind velocities on the blade section. Nevertheless, Aerodyn computes the induction factors and the corresponding aerodynamic loads assuming that those velocities are relative to an undeformed configuration [24]. The reason for this is that Aerodyn was conceived assuming small deflections on the blade section. It is important to remember that, besides these limitations of Aerodyn as an aerodynamic model, the suite Aerodyn-FAST cannot take into account coupled deformation modes (i. e. flexo-torsional, flexural-flexural, ect.) due to limitations in the structural theory used.

\subsubsection{Wind input data}

Wind input data in the FMI provides the LSR-BEM interference model updated information on the rotor incoming wind for computations of the aerodynamic loads. In order to keep retro-compatibility with existing wind databases, the model implemented in this work is the same as the one in Aerodyn [10]. This could be called a point wind file model since the wind speed at any point is calculated by shear applied to the point where wind is defined. Also note that this model does not take into account any turbulent velocity 
components [10]. The wind input data is provided by reading the pre-set file containing hub-height data history including the following parameters:

- Time

- Horizontal wind speed $\quad U_{\infty}$

- Instantaneous wind direction $\delta$

- Vertical wind speed $\quad U_{z}$

- Horizontal linear shear $\quad S h_{h l}$

- Vertical power-law shear $\quad S h_{v p}$

- Vertical linear shear $\quad S h_{v l}$

- Gust (horizontal) velocity $\quad U_{g s t}$

A difference with Aerodyn's implementation is that the time evolution of this parameters is interpolated using piecewise cubic Hermite interpolating polynomials. This provides smoother interpolations and also takes into account all the data points available compared to a standard linear interpolation.

To express the formula for the incoming wind velocity, we will need first to re-orient our blade section position $\mathrm{p}_{\mathrm{G}}$, from equation 3.56 to take into account rotor yaw effects. With this, our new position is:

$$
\mathbf{p}_{\mathbf{w}}=\mathbf{C}_{\psi}^{T} \mathbf{p}_{\mathbf{G}}
$$

Thus, the expression for the incoming hub-height wind is:

$$
\begin{aligned}
U_{h h}= & U_{\infty}\left(\frac{p w_{3}}{H_{r e f}}\right)^{S h_{h l}}+U_{\infty} \frac{S h_{h l}}{W_{r e f}} p w_{2} \cos (\delta)+p w_{1} \sin (\delta) \\
& +U_{\infty} \frac{S h_{v l}}{W_{r e f}}\left(p w_{3}-H_{r e f}\right)+U_{g s t}
\end{aligned}
$$

where $H_{\text {ref }}$ is the reference height for $U_{\infty}$ and $W_{\text {ref }}$ is a reference width, equal to the rotor diameter, to scale the linear shears. Using $U_{h h}$ we can now compute the complete wind velocity vector as:

$$
\mathbf{U} \mathbf{w}=\left[\begin{array}{c}
U_{h h} \cos (\delta) \\
-U_{h h} \sin (\delta) \\
U_{z}
\end{array}\right]
$$

Once Uw is obtained, the FMI corrects this wind velocity vector to account for rotor 
tower influences. Bak, et al. [31] developed a model for upwind rotors. This model implements a potential flow theory around a cylinder combined with a downwind wake model dependent on the tower's drag coefficient [10] modifying the local velocity field at all points around the tower. For downwind rotors, a velocity deficit due to the presence of the tower (tower shadow) is computed instead. The model based on the work of SRJ Powles [32], computes a tower wake deficit model which increase with downwind distance. Details on these models and their implementation can be found in [10,31,32].

Once the effects of the rotor tower are applied to $\mathrm{Uw}$, the FMI re-projects it back to the hub coordinate system $h$, to input the vector to the LSR-BEM interference model.

$$
\mathbf{U h}=\mathbf{C}_{\Omega} \mathbf{C}_{\tau} \mathbf{C}_{\psi} \mathbf{U w t}
$$

Here, Uwt is the wind velocity vector from equation 3.59 affected by the tower influence. The components of Uh are the ones later modified by the interference factors $a$ and $a^{\prime}$ in equation 3.20 . 


\section{The Structural Model}

Wind turbine blades are typically slender structures with high flexibility. They are usually not simple to model due to the inhomogeneous distribution of material properties and the complexity of their cross sections (see figure 4.1). Detailed 2-D shell or fully 3-D solid models can be highly expensive in computational terms if the structural problem must be solved along many timesteps. On the other hand, the ad hoc kinematic assumptions made in classical theories (like the Bernoulli or the standard Timoshenko approaches) may introduce significant errors, especially when the blade is vibrating with a wavelength shorter than its length. Complex blade geometry due to reasons of aerodynamic/mechanical design, new techniques of blade construction, and the use of new materials combine themselves to give a new dimension to the problem.

In order to obtain a fluid-structure interaction model capable of dealing with the com-

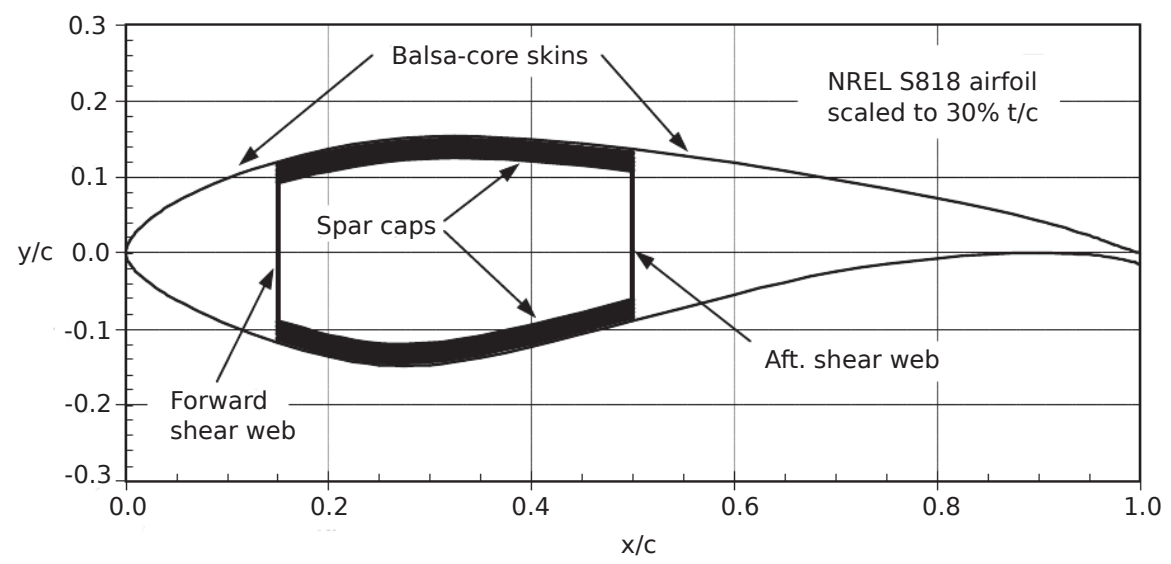

Figure 4.1. Example of blade-section structural architecture representative of current commercial blade designs. The primary structural member is a box-spar, with a substantial build-up of spar cap material between the webs. The exterior skins and internal shear webs are both sandwich construction with triaxial fiberglass laminate separated by balsa core. (adapted from [57]). 
plex features of new-generation blades, Dr. Otero developed a generalized Timoshenko code [13] based on a modified implementation of the Variational-Asymptotic Beam Sectional (VABS) model proposed and developed by Prof. Hodges and his collaborators [see 14, for example]. This model, which is able to work with curved and twisted composite beams, uses the same variables as classical Timoshenko beam theory, but the hypothesis of beam sections remaining planar after deformation is abandoned. Instead, the real warping of the deformed section is interpolated by a 2-D finite-element mesh and its contribution to the strain energy is put in terms of the classical 1-D Timoshenko's variables by means of a pre-resolution. The geometrical complexity of the blade section and/or its material inhomogeneousness are reduced into a stiffness matrix for the 1-D beam. The reduced 1-D strain energy is equivalent to the actual 3-D strain energy in an asymptotic sense. Elimination of the ad hoc kinematic assumptions produces a fully populated $6 \times 6$ symmetric matrix for the 1-D beam, with as many as 21 independent stiffnesses, instead of the six fundamental stiffnesses of the original Timoshenko theory [15]. That is why it is referred to as a generalized Timoshenko theory.

Even for the case of large displacements and rotations of the beam sections, this model allows for accurate modeling of the bending and transverse shear in two directions, extension and torsion of the blade structure as a 1-D finite-element problem. Thus, we are able to decouple a general 3-D nonlinear anisotropic elasticity problem into a linear, 2-D, cross-sectional analysis (that may be solved a priori), and a nonlinear, 1-D, beam analysis for the global problem, which is what is needed at each timestep of a fluid-structure interaction analysis. The cross-sectional 2-D analysis (that may be performed in parallel for all the cross sections along the blade) calculates the 3-D warping functions asymptotically and finds the constitutive model for the 1-D nonlinear beam analysis of the blade. After one obtains the global deformation from the 1-D beam analysis, the original 3-D fields (displacements, stresses, and strains) can be recovered a posteriori using the already-calculated 3-D warping functions.

\subsection{The Generalized Timoshenko Beam Model (GTBM)}

In order to make this chapter self-contained, we briefly outline the generalized Timoshenko theory in what follows. Referring to figure 4.2, the beam is represented by a reference line $R$ in the undeformed configuration which could be twisted and/or curved. At every point along $R$ an associated orthogonal triad $\underline{\mathbf{B}}_{1}, \underline{\mathbf{B}}_{2}, \underline{\mathbf{B}}_{3}$, is defined in such a way that $\underline{\mathbf{B}}_{1}$ is tangent to $R$ and $\underline{\mathbf{B}}_{2}, \underline{\mathbf{B}}_{3}$ are contained into the section plane which is normal to $R$. A correspondent coordinate system $\left(X^{1}, X^{2}, X^{3}\right)$ is defined where $X^{1}$ is the coordinate along $R$ and $X^{2}, X^{3}$ are the Cartesian coordinates on the section plane.

When the structure is deformed due to loading, the original reference line $R$ adopts a 




Figure 4.2. Generalized Timoshenko theory: Schematic of the reference line, orthogonal triads, and beam sections before and after deformation. 
new geometry $r$, and we have a new triad $\underline{\mathbf{t}}_{1}, \underline{\mathbf{t}}_{2}, \underline{\mathbf{t}}_{3}$ associated to each point, where $\underline{\mathbf{t}}_{1}$ is tangent to $r$ and $\underline{\mathbf{t}}_{2}, \underline{\mathbf{t}}_{3}$ are contained into the normal plane. The position of a generic point on each section in the underformed configuration may be written as

$$
\underline{\mathbf{R}}\left(X^{i}\right)=\underline{\overline{\mathbf{R}}}\left(X^{1}\right)+X^{\alpha} \underline{\mathbf{B}}_{\alpha}\left(X^{1}\right)
$$

where $\underline{\overline{\mathbf{R}}}$ denotes the position of the center of the tern along $R$, the index $\alpha$ assumes values 2 and 3 and we make use of the convention that repeated indexes are summed over their rank. The material point whose original position was given by $\underline{\mathbf{R}}\left(X^{i}\right)$ has after the deformation the position vector

$$
\underline{\mathbf{r}}\left(X^{i}\right)=\underline{\overline{\mathbf{R}}}+\underline{\mathbf{u}}+X^{\alpha} \underline{\mathbf{t}}_{\alpha}+w_{i} \underline{\mathbf{t}}_{i},
$$

where $w_{i}$ are the contribution to the displacement due to warping. Now, we are able to compute the components of the gradient-of-deformation tensor as $F_{i j}=\underline{\mathbf{t}}_{i} \cdot \mathbf{g}_{k} \underline{\mathbf{G}}^{k} \cdot \underline{\mathbf{B}}_{j}$, where $\mathbf{g}_{k}$ and $\underline{\mathbf{G}}^{k}$ are respectively the covariant base vectors for the deformed configuration and the contravariant base vectors in the undeformed configuration, obtained from the kinematic description of equations (4.1) and (4.2). The Jaumann-Biot-Cauchy strain tensor is, in this case, $\Gamma_{i j}=\frac{1}{2}\left(F_{i j}+F_{j i}\right)-\delta_{i j}$, which provides a suitable measure of the 3-D strain field in terms of the beam strain measures and arbitrary warping functions. $\Gamma$ is then used to compute the strain energy density function as

$$
2 U=\left\langle\left\langle\boldsymbol{\Gamma}^{T} \mathcal{S} \boldsymbol{\Gamma}\right\rangle\right\rangle,
$$

were, $\mathcal{S}$ is the matrix of the characteristics of the material expressed in the $\underline{\mathbf{B}}_{i}$ coordinates, and $\langle\langle\bullet\rangle\rangle=\int_{s} \bullet \sqrt{G} d X^{2} d X^{3}$, where $s$ defines integration over a cross-section and $G$ is the determinant of the metric in the undeformed base.

The next step is to find a strain energy expression asymptotically correct up to the second order of $h / l$ and $h / R_{0}$, where $h$ is the characteristic size of the section, $l$ the characteristic wavelength of deformation along the beam axis, and $R_{0}$ the characteristic radii of initial curvatures and twist of the beam. A complete second-order strain energy is sufficient for constructing a generalized Timoshenko model because, as it is generally accepted, the transverse shear strain measures are one order less than classical beam strain measures (extension, torsion and bending in two directions) [15]. A strain energy expression that asymptotically approximates the 3-D energy up to the second order is achieved using the Variational Asymptotic Method proposed in [58]. The complete derivation of this procedure is presented in [59], resulting in the following expression for the asymptotically correct strain energy:

$$
2 U=\varepsilon^{T} \mathbf{A} \varepsilon+\varepsilon^{T} 2 \mathbf{B} \varepsilon^{\prime}+\varepsilon^{\prime T} \mathbf{C} \varepsilon^{\prime}+\varepsilon^{T} 2 \mathbf{D} \varepsilon^{\prime \prime},
$$

where $\mathbf{A}, \mathbf{B}, \mathbf{C}, \mathrm{D}$ are matrices that carry information on both the geometry and the material properties of the cross section, ()$^{\prime}$ indicates the partial derivative with respect to the 
axial coordinate $X^{1}$, and $\varepsilon=\left[\begin{array}{llll}\bar{\gamma}_{11} & \bar{\kappa}_{1} & \bar{\kappa}_{2} & \bar{\kappa}_{3}\end{array}\right]^{T}$, are the strain measures defined in the classical Bernoulli beam theory: $\bar{\gamma}_{11}$ is the extension of the beam reference line, $\bar{\kappa}_{1}$ its torsion, and $\bar{\kappa}_{2}$ and $\bar{\kappa}_{3}$ the bending of the reference line in axes 2 and 3 due to the deformation.

The variational asymptotic procedure to get the matrices in equation (4.4) involves the discretization by finite-element techniques of the warping functions $w_{i}$ defined in (4.2). To this end, four constraints are imposed on $w_{i} .\left\langle w_{i}\right\rangle=0$ and $\left\langle X^{2} w_{3}-X^{3} w_{2}\right\rangle=0$, where $\langle\bullet\rangle=\int_{s} \bullet d X^{2} d X^{3}$, are intended to eliminate four rigid modes of displacement of the warped section (i.e. the three linear displacements plus rotation around $\underline{\mathbf{t}}_{1}$ ), which are already included in the Bernoulli strain measures $\varepsilon$.

For the implementation, Dr. Otero uses the classical Lagrangian-multiplier technique to impose the constraints and solve the expanded system for the constrained variational formulation itself [13]. This simplifies the procedure by basically combining the whole solution in a single step. This simplification produces by itself a certain reduction in the overall computational cost, but most importantly, it has the advantage of allowing the use of the internal-node condensation technique in the finite-element discretization. As we shall see later, internal-node condensation allows us to substantially improve the efficiency of our solution by the tri-quadrilateral finite-element technique.

Expression (4.4) for the strain energy is asymptotically correct. Nevertheless, it is difficult to use in practice because it contains derivatives of the classical strain measures, which requires complicated boundary conditions. But, the well known Timoshenko beam theory is free from such drawbacks. Hence, the next step is to fit the strain energy in (4.4) into a generalized Timoshenko model of the form

$$
2 U=\left[\begin{array}{ll}
\boldsymbol{\epsilon}^{T} & \boldsymbol{\gamma}_{s}^{T}
\end{array}\right]\left[\begin{array}{cc}
\mathbf{X} & \mathbf{Y} \\
\mathbf{Y}^{T} & \mathbf{G}
\end{array}\right]\left[\begin{array}{c}
\boldsymbol{\epsilon} \\
\boldsymbol{\gamma}_{s}
\end{array}\right]=\boldsymbol{\epsilon}^{T} \mathbf{X} \boldsymbol{\epsilon}+2 \boldsymbol{\epsilon}^{T} \mathbf{Y} \boldsymbol{\gamma}_{s}+\boldsymbol{\gamma}_{s}^{T} \mathbf{G} \boldsymbol{\gamma}_{s},
$$

where $\boldsymbol{\epsilon}=\left[\begin{array}{llll}\gamma_{11} & \kappa_{1} & \kappa_{2} & \kappa_{3}\end{array}\right]^{T}$ are the classical Timoshenko strain measures due to extension, torsion and bending, and $\gamma_{s}=\left[\begin{array}{ll}2 \gamma_{12} & 2 \gamma_{13}\end{array}\right]^{T}$ the transverse shear strains. $\mathbf{X}$, $\mathrm{Y}$ and $\mathrm{G}$ are found in such a way that the strain energy in (4.4) and (4.5) is equivalent up to at least second order [see 13, for more detail].

Finally, a stiffness matrix for the 1-D beam problem $\overline{\mathbb{S}}$ is formed as a simple reordering of the matrix $\left[\begin{array}{cc}\mathbf{X} & \mathbf{Y} \\ \mathbf{Y}^{T} & \mathbf{G}\end{array}\right]$, in such a way as to get a functional for the strain energy density of expression (4.5)

$$
2 U=\bar{\gamma}^{T} \overline{\mathbb{S}} \bar{\gamma}
$$

where $\bar{\gamma}=\left[\begin{array}{l}\gamma \\ \kappa\end{array}\right]$ is the array of Timoshenko measures of deformation regrouped in a more convenient way, $\boldsymbol{\gamma}^{T}=\left[\begin{array}{lll}\gamma_{11} & 2 \gamma_{12} & 2 \gamma_{13}\end{array}\right]$ and $\boldsymbol{\kappa}^{T}=\left[\begin{array}{lll}\kappa_{1} & \kappa_{2} & \kappa_{3}\end{array}\right]$. 
For the discretization of the 2-D sections, we adopted the tri-quadrilateral finite-element technique, which is based on the use of nine-node biquadratic isoparametric finite elements that possess a high convergence rate and, due to their biquadratic interpolation of the geometric coordinates, provide the additional ability of reducing the so-called skin-error on curvilinear boundaries when compared to linear elements. For details see Bathe [60].

In order to combine the advantages of the nine-node quadrilateral isoparametric element with the geometrical ability of a triangular grid to create suitable non-structured meshes with gradual and smooth changes of mesh density, we implemented what we called tri-quadrilateral isoparametric elements. The tri-quadrilateral elements consist of an assembly of three quadrilateral nine-node isoparametric elements in which each triangle of a standard unstructured mesh is divided into. By static condensation of the nodes that lie inside the triangle, we can significantly reduce the number of nodes to solve in the final system, subsequently recovering the values for the internal nodes from the solution on the non-condensable nodes. The internal nodes may be expressed in terms of nodes which lay on the elemental boundary following the classical procedure for elemental condensation [60]. This process of condensation allows us to reduce the size of the new system to approximately $40 \%$ of the original system. The use of the static condensation procedure is attractive not only because it reduces the size of the stiffness matrices arising in finiteelement and spectral-element methods but also because it improves the condition number of the final condensed system. For details see Ponta [20].

\subsection{The 1D model}

To solve the one-dimensional problem for the equivalent beam, we use a formulation based on the intrinsic equations for the beam obtained from variational principles (see [61]), and weighted in an energy-consistent way according to Patil and Althoff [62], which produces the following variational formulation:

$$
\begin{array}{r}
\int_{0}^{\ell}[\underbrace{\delta \overline{\mathbf{V}}^{T} \overline{\mathbb{I}} \dot{\overline{\mathbf{V}}}}_{1}+\underbrace{\left.\delta \overline{\mathbf{F}}^{T} \overline{\mathbb{S}}^{-1} \dot{\overline{\mathbf{F}}}\right] d X^{1}}_{2}=\int_{0}^{\ell}[\underbrace{\delta \overline{\mathbf{V}}^{T} \overline{\mathbf{F}}^{\prime}+\underbrace{\delta \overline{\mathbf{V}}^{T} \hat{\mathbf{K}} \overline{\mathbf{F}}}_{4}+\underbrace{\delta \overline{\mathbf{V}}^{T} \hat{\gamma} \overline{\mathbf{F}}}_{5}+}_{3} \\
\underbrace{\delta \overline{\mathbf{V}}^{T} \overline{\mathbf{f}}}_{6}-\underbrace{\delta \overline{\mathbf{V}}^{T} \hat{\mathbf{V}} \overline{\mathbb{I}} \overline{\mathbf{V}}}_{7}+\underbrace{\delta \overline{\mathbf{F}}^{T} \overline{\mathbf{V}}^{\prime}}_{8}-\underbrace{\delta \overline{\mathbf{F}}^{T} \hat{\mathbf{K}}^{T} \overline{\mathbf{V}}}_{9}-\underbrace{\delta \overline{\mathbf{F}}^{T} \hat{\gamma}^{T} \overline{\mathbf{V}}}_{10}] d X^{1},
\end{array}
$$

where

$$
\begin{array}{cc}
\overline{\mathbf{F}}=\left[\begin{array}{c}
\mathbf{F} \\
\mathbf{M}
\end{array}\right], \quad \overline{\mathbf{V}}=\left[\begin{array}{c}
\mathbf{V} \\
\boldsymbol{\Omega}
\end{array}\right], \quad \overline{\mathbf{f}}=\left[\begin{array}{c}
\mathbf{f} \\
\mathbf{m}
\end{array}\right], \\
\hat{\gamma}=\left[\begin{array}{cc}
\tilde{\boldsymbol{\kappa}} & \mathbf{0} \\
\tilde{\gamma} & \tilde{\boldsymbol{\kappa}}
\end{array}\right], \quad \hat{\mathbf{V}}=\left[\begin{array}{cc}
\tilde{\Omega} & 0 \\
\tilde{\mathbf{V}} & \tilde{\Omega}
\end{array}\right], \quad \hat{\mathbf{K}}=\left[\begin{array}{cc}
\tilde{\mathbf{K}} & 0 \\
\tilde{\mathbf{e}_{1}} & \tilde{\mathbf{K}}
\end{array}\right] .
\end{array}
$$


Tilde indicates the skew-symmetric matrix associated with a vector magnitude in such a way that, for example, if we have any pair of vectors $\mathbf{A}$ and $\mathbf{B}$, the matrix-vector product $\tilde{\mathbf{A}} \mathbf{B}$ is equivalent to the cross product $\mathbf{A} \times \mathbf{B}$. Thus, $\tilde{\gamma}$ is associated with $\gamma, \tilde{\boldsymbol{\kappa}}$ with $\boldsymbol{\kappa}, \tilde{\mathbf{V}}$ with $\mathbf{V}$, and so forth. Hence, matrix $\hat{\gamma}$ is a rearrangement of the components of the strainmeasures vector $\bar{\gamma}$ defined above, while the generalized-velocities vector $\overline{\mathbf{V}}$ and matrix $\hat{\mathbf{V}}$ represent the components of the linear and angular velocities, and matrix $\hat{\mathbf{K}}$ represents the initial torsion and curvatures of the beam (matrix $\tilde{\mathbf{e}_{1}}$ is the skew-symmetric matrix associated with $\mathbf{e}_{1}^{T}=\left[\begin{array}{ccc}1 & 0 & 0\end{array}\right]$, the unit vector along $\left.X^{1}\right)$. The generalized-forces vector $\overline{\mathbf{F}}$ represents the forces and moments related with the strain measures $\left(\bar{\gamma}=\overline{\mathbb{S}}^{-1} \overline{\mathbf{F}}\right)$, and the generalized-distributed-loads vector $\overline{\mathbf{f}}$ represents the forces and moments distributed along the axis of the beam. Here, $\overline{\mathbb{S}}$ is the stiffness matrix for the 1-D model corresponding to equation (4.6); and $\overline{\mathbb{I}}$ is the inertia matrix of each section. The upper dot indicates a time derivative, and the prime a derivative with respect to the longitudinal coordinate of the beam $X^{1}$.

This variational formulation was discretized by the spectral-element method (see $[63,64])$. The magnitudes in (4.7) where replaced by their interpolated counterparts: $\overline{\mathbf{V}}=\mathbf{H}_{\overline{\mathbf{V}}}^{e} \mathbf{Q}^{e}$, and $\overline{\mathbf{F}}=\mathbf{H}_{\overline{\mathbf{F}}}^{e} \mathbf{Q}^{e}$, where $\mathbf{H}_{\overline{\mathbf{V}}}^{e}$ and $\mathbf{H}_{\overline{\mathbf{F}}}^{e}$ are the interpolation-function arrays, and $\mathrm{Q}^{e}$ is a vector containing the nodal values of both the generalized velocities and the generalized forces. Superscript $e$ indicates discretization of the terms at the elemental level, which will disappear after the final assembly of the terms into the global matrix for the whole beam. The axial derivatives of the magnitudes were interpolated in a similar way: $\overline{\mathbf{V}}^{\prime}=\mathbf{B}_{\overline{\mathbf{V}}}^{e} \mathbf{Q}^{e}$, and $\overline{\mathbf{F}}^{\prime}=\mathbf{B}_{\overline{\mathbf{F}}}^{e} \mathbf{Q}^{e}$, where $\mathbf{B}_{\overline{\mathbf{V}}}^{e}$ and $\mathbf{B}_{\overline{\mathbf{F}}}^{e}$ are the arrays for the interpolationfunction derivatives. Then, the following discretized version of (4.7) is obtained:

$$
\delta \mathbf{Q}^{e T} \mathbf{M}_{\mathbf{1}}^{e} \dot{\mathbf{Q}}^{e}=\delta \mathbf{Q}^{e T}\left(\mathbf{K}_{\mathbf{1}}^{e}+\mathbf{K}_{\mathbf{2}}^{e}\right) \mathbf{Q}^{e}+\delta \mathbf{Q}^{e T} \mathbf{K}_{\mathbf{q}}^{e} \overline{\mathbf{q}}^{e}+\delta \mathbf{Q}^{e T} \mathbf{B}_{Q}^{e}\left(\mathbf{Q}^{e}\right)
$$

where

$$
\begin{aligned}
& \mathbf{M}_{\mathbf{1}}^{e}=\int_{-1}^{1}\left[\mathbf{H}_{\overline{\mathbf{V}}}^{e T} \overline{\mathbb{I}} \mathbf{H}_{\overline{\mathbf{V}}}^{e}+\mathbf{H}_{\overline{\mathbf{F}}}^{e^{T}} \overline{\mathbb{S}}^{-1} \mathbf{H}_{\overline{\mathbf{F}}}^{e}\right] J d t, \\
& \mathbf{K}_{\mathbf{1}}^{e}=\int_{-1}^{1}\left[\mathbf{H}_{\overline{\mathbf{V}}}^{e T} \mathbf{B}_{\overline{\mathbf{F}}}^{e}+\mathbf{H}_{\overline{\mathbf{F}}}^{e T} \mathbf{B}_{\overline{\mathbf{V}}}^{e}\right] J d t \mathbf{Q}^{e}, \\
& \mathbf{K}_{\mathbf{2}}^{e}=\int_{-1}^{1}\left[\mathbf{H}_{\overline{\mathbf{V}}}^{e T} \hat{\mathbf{K}} \mathbf{H}_{\overline{\mathbf{F}}}^{e}-\mathbf{H}_{\overline{\mathbf{F}}}^{e} \hat{\mathbf{K}}^{T} \mathbf{H}_{\overline{\mathbf{V}}}^{e}\right] J d t, \\
& \mathbf{K}_{\mathbf{q}}^{e}=\int_{-1}^{1} \mathbf{H}_{\overline{\mathbf{V}}}^{e}{ }^{T} \mathbf{H}_{\overline{\mathbf{F}}}^{e} J d t .
\end{aligned}
$$

$\mathbf{M}_{1}^{e}$ corresponds to the discretization of terms 1 and 2 giving the equivalent of a mass matrix. $\mathbf{K}_{1}^{e}$, corresponding to terms 3 and 8 , is the stiffness matrix of the 1-D problem. $\mathbf{K}_{2}^{e}$, corresponding to terms 4 and 9 , is the additional stiffness related with the twist and curvature of the undeformed configuration. $\mathbf{K}_{\mathbf{q}}^{e}$ corresponds to the evaluation of term 6 , 
the contribution of the distributed loads; and $\overline{\mathbf{q}}^{e}$ is an array containing the nodal values of the generalized distributed loads. $t$ is the natural coordinate in the elements and $J$ is the Jacobian of the mapping from the problem coordinate $X^{1}$ to $t$ (see [60]). The discretized version of the terms in (4.7) related to non-linear interactions, i.e. terms 5, 7 and 10, gives

$$
\mathbf{B}_{Q}^{e}\left(\mathbf{Q}^{e}\right)=\int_{-1}^{1}\left[\mathbf{H}_{\overline{\mathbf{V}}}^{e^{T}} \hat{\gamma} \mathbf{H}_{\overline{\mathbf{F}}}^{e}-\mathbf{H}_{\overline{\mathbf{V}}}^{e^{T}} \hat{\mathbf{V}} \overline{\mathbb{I}} \mathbf{H}_{\overline{\mathbf{V}}}^{e}-\mathbf{H}_{\overline{\mathbf{F}}}^{e^{T}} \hat{\gamma}^{T} \mathbf{H}_{\overline{\mathbf{V}}}^{e}\right] \mathbf{Q}^{e} J d t
$$

After the assembly of the elemental terms into the global system, we obtain the differential equations for the solution of the 1-D nonlinear problem of the equivalent beam mentioned in section 2.1:

$$
\dot{\mathbf{Q}}=\mathbf{M}_{\mathbf{1}}^{-1}\left[\left(\mathbf{K}_{\mathbf{1}}+\mathbf{K}_{\mathbf{2}}\right) \mathbf{Q}+\mathbf{K}_{\mathbf{q}} \overline{\mathbf{q}}+\mathbf{B}_{Q}(\mathbf{Q})\right]
$$

As mentioned in section 2.1, the frequencies and linear vibrational modes around a steady-state solution may be obtained by linearizing (4.9). A linearization of the nonlinear term $\mathbf{B}_{Q}^{e}\left(\mathbf{Q}^{e}\right)$ around any given configuration $\mathbf{Q}_{1}^{e}$ gives the matrix

$$
\begin{aligned}
\mathbf{K}_{\mathbf{N}}^{e}\left(\mathbf{Q}_{1}^{e}\right)= & \int_{-1}^{1}\left\{\mathbf{H}_{\overline{\mathbf{V}}}^{e T}\left[\hat{\gamma}_{1} \mathbf{H}_{\overline{\mathbf{F}}}^{e}-\hat{\mathbf{V}}_{1} \overline{\mathbb{I}} \mathbf{H}_{\overline{\mathbf{V}}}^{e}-\hat{\mathbf{F}}_{1} \overline{\mathbb{S}}^{-1} \mathbf{H}_{\overline{\mathbf{F}}}^{e}+\hat{\mathbf{P}}_{1} \mathbf{H}_{\overline{\mathbf{V}}}^{e}\right]+\right. \\
& \left.\mathbf{H}_{\overline{\mathbf{F}}}^{e T}\left[\hat{\mathbf{V}}_{1}^{T} \overline{\mathbb{S}}^{-1} \mathbf{H}_{\overline{\mathbf{F}}}^{e}-\hat{\gamma}_{1}^{T} \mathbf{H}_{\overline{\mathbf{V}}}^{e}\right]\right\} J d t
\end{aligned}
$$

where

$$
\hat{\mathbf{F}}=\left[\begin{array}{cc}
\mathbf{0} & \tilde{\mathbf{F}} \\
\tilde{\mathbf{F}} & \tilde{\mathbf{M}}
\end{array}\right], \quad \hat{\mathbf{P}}=\left[\begin{array}{cc}
\mathbf{0} & \tilde{\mathbf{P}}_{v} \\
\tilde{\mathbf{P}}_{v} & \tilde{\mathbf{P}}_{\omega}
\end{array}\right]
$$

Matrix $\hat{\mathbf{F}}$ is a rearrangement of the components of the generalized-forces vector $\overline{\mathbf{F}}$ defined above. Matrix $\hat{\mathbf{P}}$ is a rearrangement of the components of the generalized-momentum vector $\overline{\mathbf{P}}=\left[\begin{array}{l}\mathbf{P}_{v} \\ \mathbf{P}_{\omega}\end{array}\right]$, which represents the linear and angular momenta related with the generalized-velocities $(\overline{\mathbf{P}}=\overline{\mathbb{I}} \overline{\mathbf{V}})$. Tilde operates in the same way defined before, and the subscript 1 indicates the value of the magnitudes at a given state $\mathbf{Q}_{1}^{e}$.

Finally, after the assembly of the elemental terms into the global system, the solution for the nonlinear problem (4.7) in its steady state was obtained by solving iteratively for 
$\Delta \mathrm{Q}$ the discretized expression

$$
\left[\mathbf{K}_{\mathbf{1}}+\mathbf{K}_{\mathbf{2}}+\mathbf{K}_{\mathbf{N}}\left(\mathbf{Q}^{(i)}\right)\right] \Delta \mathbf{Q}=-\mathbf{K}_{\mathbf{q}} \overline{\mathbf{q}}-\left(\mathbf{K}_{\mathbf{1}}+\mathbf{K}_{\mathbf{2}}\right) \mathbf{Q}^{(i)}-\mathbf{B}_{Q}\left(\mathbf{Q}^{(i)}\right),
$$

and updating the global vector of nodal values of the generalized velocities and forces as $\mathrm{Q}^{(i+1)}=\mathrm{Q}^{(i)}+\Delta \mathrm{Q}$.

From the steady-state solution we also obtain the vibrational modes of the blade structure and their corresponding frequencies by solving the eigenvalue problem

$$
\mathbf{M}_{\mathbf{1}} \dot{\mathbf{Q}}+\left[\mathbf{K}_{\mathbf{1}}+\mathbf{K}_{\mathbf{2}}+\mathbf{K}_{\mathbf{N}}\left(\mathbf{Q}^{(i)}\right)\right] \mathbf{Q}=\mathbf{0} .
$$

From these results for the intrinsic equations we recovered the displacements and rotations of the blade sections by solving the kinematic equations for the beam (see [14])

$$
\begin{array}{r}
\mathbf{u}^{\prime}-\mathbf{C}_{r R}^{T}\left(\boldsymbol{\gamma}+\mathbf{e}_{1}\right)+\mathbf{e}_{1}+\tilde{\mathbf{K}} \mathbf{u}=\mathbf{0} \\
\tilde{\mathbf{K}}+\tilde{\boldsymbol{\kappa}}+\mathbf{C}_{r R}^{\prime} \mathbf{C}_{r R}^{T}-\mathbf{C}_{r R} \tilde{\mathbf{K}} \mathbf{C}_{r R}^{T}=\mathbf{0}
\end{array}
$$

where $\mathbf{u}$ is the vector of displacements of each point along the reference line from its position in the reference configuration to the one in the deformed configuration, and $\mathbf{C}_{r R}$ is the orthogonal matrix that rotates the local triad from its original orientation in the reference configuration to the one in the deformed configuration (both are defined in function of the longitudinal coordinate $X^{1}$ ). The strains $\gamma$ and $\kappa$ were computed from the generalized forces and the stiffness of the corresponding blade section. Equations 4.12 and 4.13 were also linearized, and like the other expressions, discretized by the spectral-element method.

\subsection{Constitutive relations of composite materials}

Composite materials show nonlinear stress-strain relations due to the behavior of the different combined materials under load. Despite this fact, when deformations are small, this behavior may be equivalent to a Hookean material. For the purposes of this thesis work we will assume that the materials in the blades don't get to reach the failure state. Thus, our hypothesis will be that materials work under the linear-elastic regime being an adequate hypothesis to model the materials behavior before they get to fail due to delamination or fiber failure.

Blade materials working under the linear-elastic regime can be classified as orthotropic, transversely isotropic, or linear isotropic materials. Specific structural properties for the blades can be determined during manufacturing through the fibers orientation. Therefore, in this section we will show the constitutive relations for each of the above mentioned 
cases and how they get transformed for different coordinate systems (see [65-68]). Given the second order tensors $\underline{\boldsymbol{e}}$ for strain and $\underline{\boldsymbol{s}}$ for stress, for a material under the Hooke law, there would be a fourth order tensor $\underline{\mathcal{S}}$, called the constitutive tensor, such that:

$$
\underline{s}=\underline{\mathcal{S}}: \underline{\boldsymbol{e}},
$$

or given a certain base, in components:

$$
s_{i j}=\mathcal{S}_{i j k l} e_{k l}
$$

Generally a second order tensor is defined by nine independent constants while a fourth order tensor has eighty-one. Stress and strain tensors are symmetric, thus, instead of nine independent constants they only have six. Due to this and the fact that the quadratic form of the deformation energy given by $\underline{\boldsymbol{e}}: \underline{\boldsymbol{s}}$, the constitutive tensor has the following symmetry when we express its components in a specific base:

$$
\mathcal{S}_{i j k l}=\mathcal{S}_{j i k l}=\mathcal{S}_{i j l k}=\mathcal{S}_{k l i j}
$$

That is why the number of independent constants is reduced to twenty-one for the most general case.

On the other hand, and taking advantage of the tensor's symmetry, it is common to write the constitutive relation in equation 4.14 in a more convenient matrix form such as:

$$
\mathrm{s}=\mathcal{S e}
$$

where the arrays and matrices in 4.17 can be expressed as:

$$
\mathbf{s}=\left[\begin{array}{c}
s_{11} \\
s_{22} \\
s_{33} \\
s_{23} \\
s_{31} \\
s_{12}
\end{array}\right], \mathcal{S}=\left[\begin{array}{llllll}
\mathcal{S}_{1111} & \mathcal{S}_{1122} & \mathcal{S}_{1133} & \mathcal{S}_{1123} & \mathcal{S}_{1131} & \mathcal{S}_{1112} \\
\mathcal{S}_{1122} & \mathcal{S}_{2222} & \mathcal{S}_{2233} & \mathcal{S}_{2223} & \mathcal{S}_{2231} & \mathcal{S}_{2212} \\
\mathcal{S}_{1133} & \mathcal{S}_{2233} & \mathcal{S}_{3333} & \mathcal{S}_{3323} & \mathcal{S}_{3331} & \mathcal{S}_{3312} \\
\mathcal{S}_{1123} & \mathcal{S}_{2223} & \mathcal{S}_{3323} & \mathcal{S}_{2323} & \mathcal{S}_{2331} & \mathcal{S}_{2312} \\
\mathcal{S}_{1131} & \mathcal{S}_{2231} & \mathcal{S}_{3331} & \mathcal{S}_{2331} & \mathcal{S}_{3131} & \mathcal{S}_{3112} \\
\mathcal{S}_{1112} & \mathcal{S}_{2212} & \mathcal{S}_{3312} & \mathcal{S}_{2312} & \mathcal{S}_{3112} & \mathcal{S}_{1212}
\end{array}\right] \quad \mathbf{e}=\left[\begin{array}{c}
e_{11} \\
e_{22} \\
e_{33} \\
2 e_{23} \\
2 e_{31} \\
2 e_{12}
\end{array}\right]
$$

Matrix $\mathcal{S}$ is called the stiffness matrix and the inverse, $\mathcal{C}=\mathcal{S}^{-1}$, is known as the compliance matrix. It is possible to write the components of matrices $\mathcal{S}$ and $\mathcal{C}$ as a function 
of the mechanical properties of the materials: Elasticity modulus, Poisson coefficients, and shear modulus, making the expressions of the compliance matrix more compact. Thus, it is usually more convenient to obtain the expression of this matrix and then invert it in case the stiffness matrix is needed.

\section{Orthotropic materials}

An orthotropic material has three main symmetry planes so its mechanical properties are, in general, different along each axis. Orthotropic materials are thus anisotropic; with their properties depending on the direction in which they are measured. A special characteristic of this materials is that stresses applied perpendicularly to the symmetry planes don't generate shear stresses. An example of these materials could be laminates with perpendicularly crossing fibers. This type of materials are of great interest for wind turbine blades manufacture. Using a Cartesian tern perpendicularly aligned with the symmetry planes $(x, y, z)$, the constitutive tensor $\mathcal{S}$ can be expressed as:

$$
\mathcal{S}=\left[\begin{array}{cccccc}
\mathcal{S}_{x x x x} & \mathcal{S}_{x x y y} & \mathcal{S}_{x x z z} & 0 & 0 & 0 \\
\mathcal{S}_{x x y y} & \mathcal{S}_{y y y y} & \mathcal{S}_{y y z z} & 0 & 0 & 0 \\
\mathcal{S}_{x x z z} & \mathcal{S}_{y y z z} & \mathcal{S}_{z z z z} & 0 & 0 & 0 \\
0 & 0 & 0 & \mathcal{S}_{y z y z} & 0 & 0 \\
0 & 0 & 0 & 0 & \mathcal{S}_{z x z x} & 0 \\
0 & 0 & 0 & 0 & 0 & \mathcal{S}_{x y x y}
\end{array}\right]
$$

The coefficients of $\mathcal{S}$ can be rewritten from a continuous mechanics standpoint by using the elasticity modulus $\left(E_{x}, E_{y}, E_{z}\right)$, the Poisson coefficients $\left(\nu_{x y}, \nu_{y x}, \nu_{z x}, \nu_{x z}, \nu_{y z}, \nu_{z y}\right)$ and the shear modulus $\left(G_{x y}, G_{y z}, G_{z x}\right)$. Thus, we arrive at a more simple expression of the compliance matrix according to [69]:

$$
\mathcal{C}=\left[\begin{array}{cccccc}
\frac{1}{E_{x}} & -\frac{\nu_{y x}}{E_{y}} & -\frac{\nu_{z x}}{E_{z}} & 0 & 0 & 0 \\
-\frac{\nu_{x y}}{E_{x}} & \frac{1}{E_{y}} & -\frac{\nu_{z y}}{E_{z}} & 0 & 0 & 0 \\
-\frac{\nu_{x z}}{E_{x}} & -\frac{\nu_{y z}}{E_{y}} & \frac{1}{E_{z}} & 0 & 0 & 0 \\
0 & 0 & 0 & \frac{1}{G_{y z}} & 0 & 0 \\
0 & 0 & 0 & 0 & \frac{1}{G_{z x}} & 0 \\
0 & 0 & 0 & 0 & 0 & \frac{1}{G_{x y}}
\end{array}\right]
$$

From this expression and due to the symmetry of the compliance matrix, one can identify the following identities: 


$$
\frac{\nu_{y x}}{E_{y}}=\frac{\nu_{x y}}{E_{x}}, \quad \frac{\nu_{z x}}{E_{z}}=\frac{\nu_{x z}}{E_{x}}, \quad \frac{\nu_{z y}}{E_{z}}=\frac{\nu_{y z}}{E_{y}},
$$

where we can clearly see that the constitutive properties of orthotropic materials are defined by nine independent constants: three elasticity modulus, three shear modulus, and three out of the six Poisson coefficients. These are the parameters frequently reported as material properties in the literature.

In the same way, the stiffness matrix could be rewritten as a function of these nine constants; nevertheless, it is a more convenient practice to compute it as the inverse of the compliance matrix, from equation 4.20.

\section{Transversely isotropic materials}

Transversely isotropic materials have one axis of symmetry. We could mention as an example the honeycomb type of materials employed in the manufacturing of sandwich composites. In this laminates, the fibers are oriented in the same direction as the symmetry axis.

Matrix $\mathcal{S}$, from equation 4.17 , has the same expression as for orthotropic materials. Due to the restrictive symmetry, this type of materials could be characterized through five independent coefficients: $\alpha, \beta, \gamma, \lambda$ and $G$ (see for example [68]) such that the components of the constitutive tensor can be expresses as:

$$
\begin{aligned}
\mathcal{S}_{i j k l}= & \lambda \delta_{i j} \delta_{k l}+G\left(\delta_{i k} \delta_{j l}+\delta_{i l} \delta_{j k}\right)+\alpha\left(\delta_{i j} a_{k} a_{l}+\delta_{k l} a_{i} a_{j}\right) \\
& +\beta\left(\delta_{i l} a_{k} a_{j}+\delta_{j k} a_{i} a_{l}+\delta_{i k} a_{j} a_{l}+\delta_{j l} a_{i} a_{k}\right)+\gamma\left(a_{i} a_{j} a_{k} a_{l}\right),
\end{aligned}
$$

where $\delta_{i j}$ is the Kroneker delta and $\underline{\mathbf{a}}=a_{i} \underline{\mathbf{B}}_{i}$ is the symmetry axis direction expressed in a vector base $\underline{\mathbf{B}}_{i}$.

Being $x$ the direction of the symmetry axis, the components of the compliance matrix can be also expressed as in equation 4.20. Due to the more restrictive symmetry and using the identities in equation 4.21, we obtain:

$$
E_{z}=E_{y}, \quad \nu_{z x}=\nu_{y x}, \quad G_{y z}=\frac{E_{y}}{2\left(1+\nu_{z y}\right)}, \quad G_{x z}=G_{x y} \text {. }
$$

Thus, the constitutive properties of the transversely isotropic materials are defined by 
five independent material constants: Two elasticity modulus $\left(E_{x}, E_{y}\right)$, two Poisson coefficients $\left(\nu_{y x}, \nu_{z y}\right)$ and the shear modulus $G_{x y}$. With this, the compliance matrix can be expressed as:

$$
\mathcal{C}=\left[\begin{array}{cccccc}
\frac{1}{E_{x}} & -\frac{\nu_{y x}}{E_{y}} & -\frac{\nu_{y x}}{E_{y}} & 0 & 0 & 0 \\
-\frac{\nu_{y x}}{E_{y}} & \frac{1}{E_{y}} & -\frac{\nu_{z y}}{E_{y}} & 0 & 0 & 0 \\
-\frac{\nu_{y x}}{E_{y}} & -\frac{\nu_{z y}}{E_{y}} & \frac{1}{E_{y}} & 0 & 0 & 0 \\
0 & 0 & 0 & \frac{2\left(1+\nu_{z y}\right)}{E_{y}} & 0 & 0 \\
0 & 0 & 0 & 0 & \frac{1}{G_{x y}} & 0 \\
0 & 0 & 0 & 0 & 0 & \frac{1}{G_{x y}}
\end{array}\right]
$$

and the corresponding stiffness matrix can be computed by inverting $\mathcal{C}$ just like in the previous case.

\section{Isotropic materials}

Isotropic materials are generally defined by five material constants which define the components of the constitutive tensor. This constants are: the Young modulus $(E)$, the shear modulus $(G)$, the volumetric modulus $(K)$, the Poisson coefficient $(\nu)$ and the Lamé coefficient $(\lambda)$. As they are not independent, two constants are needed to define the other three [65]. The five coefficients are related by the following identities:

$$
\begin{aligned}
\lambda & =\frac{\nu E}{(1+\nu)(1-2 \nu)}=\frac{2 \nu G}{1-2 \nu}, \\
K & =\frac{E}{3(1-2 \nu)}=\lambda+\frac{2}{3} G, \\
G & =\frac{E}{2(1+\nu)}, \\
E & =\frac{(3 \lambda+2 G)}{(\lambda+G)} G, \\
\nu & =\frac{\lambda}{2(\lambda+G)} .
\end{aligned}
$$

Typically for this type of materials, the coefficients of the stiffness matrix are expressed as a function of the Young modulus and the Poisson coefficient. In this case, the expression for the stiffness matrix is: 


$$
\mathcal{S}=\left[\begin{array}{cccccc}
\frac{E(1-\nu)}{(1+\nu)(1-2 \nu)} & \frac{E \nu}{(1+\nu)(1-2 \nu)} & \frac{E \nu}{(1+\nu)(1-2 \nu)} & 0 & 0 & 0 \\
\frac{E \nu}{(1+\nu)(1-2 \nu)} & \frac{E(1-\nu)}{(1+\nu)(1-2 \nu)} & \frac{E \nu}{(1+\nu)(1-2 \nu)} & 0 & 0 & 0 \\
\frac{E \nu}{(1+\nu)(1-2 \nu)} & \frac{E \nu}{(1+\nu)(1-2 \nu)} & \frac{E(1-\nu)}{(1+\nu)(1-2 \nu)} & 0 & 0 & 0 \\
0 & 0 & 0 & \frac{E}{2(1+\nu)} & 0 & 0 \\
0 & 0 & 0 & 0 & \frac{E}{2(1+\nu)} & 0 \\
0 & 0 & 0 & 0 & 0 & \frac{E}{2(1+\nu)}
\end{array}\right]
$$

and its compliance matrix can be expressed as:

$$
\mathcal{C}=\left[\begin{array}{cccccc}
\frac{1}{E} & -\frac{\nu}{E} & -\frac{\nu}{E} & 0 & 0 & 0 \\
-\frac{\nu}{E} & \frac{1}{E} & -\frac{\nu}{E} & 0 & 0 & 0 \\
-\frac{\nu}{E} & -\frac{\nu}{E} & \frac{1}{E} & 0 & 0 & 0 \\
0 & 0 & 0 & \frac{1}{G} & 0 & 0 \\
0 & 0 & 0 & 0 & \frac{1}{G} & 0 \\
0 & 0 & 0 & 0 & 0 & \frac{1}{G}
\end{array}\right]
$$

\section{Change of basis of the stiffness and compliance matrices}

The stiffness and compliance matrices of the above-mentioned materials are formed by the components of the constitutive tensor. This components are expressed in basis related with the structure of the materials (i.e. axis and planes of symmetry). As the basis defined for the material usually differ from the one defined for the structural problem, one might reorient the matrix components by a change of basis operation. This change of basis is similar to those already explained in section 3.3.2, but instead of re-projecting a vector, we now apply the rotation matrices to modify the orientation of the coordinate system.

Given a constitutive tensor $\mathcal{S}_{i j k l}$ with components originally expressed in the base $\underline{\mathbf{b}}_{p}$, we can express the same tensor now with components $\hat{\mathcal{S}}_{a b c d}$ expressed in the base $\underline{\mathbf{b}}_{q}$ by:

$$
\hat{\mathcal{S}}_{a b c d}=\mathcal{S}_{i j k l} \hat{C}_{i a} \hat{C}_{j b} \hat{C}_{k c} \hat{C}_{l d}
$$

where $\hat{C}_{m n}$ are the director cosines of the change of basis matrix which rotates from $\underline{\mathbf{b}}_{p}$ to $\hat{\mathbf{b}}_{q}$ such that:

$$
\underline{\mathbf{b}}_{q}=\hat{C}_{q p} \underline{\mathbf{b}}_{p} \text {. }
$$


This operation could be done in two ways. The first one is by operating directly with the components of the constitutive tensor and the linear operators as described in equation 4.27. The second is to create a unique matrix to do the analogous operations directly on the stiffness matrix. In this work we chose to implement the first option.

Laminates are built out of various layers which could differ in materials and orientation. Two terns are usually employ to define the laminate. First, a $\underline{\mathbf{r}}_{i}$ tern, characteristic of each layer, which defines the orientation of constitutive tensor, being $\underline{\mathbf{r}}_{3}$ perpendicular to the layer. It is also common to express the three principal directions with letters $l, t$ and $n$ redefining $\underline{\mathbf{r}}_{i}$ as: $\underline{\mathbf{r}}_{1}=\underline{\mathbf{e}}_{l}, \underline{\mathbf{r}}_{2}=\underline{\mathbf{e}}_{t}$ and $\underline{\mathbf{r}}_{3}=\underline{\mathbf{e}}_{n}$. Letters $l, t$ and $n$ refer to the longitudinal, traverse and normal directions to the layer respectively. On the other hand, a $\mathbf{y}_{i}$ tern is defined for the laminate, where vector $\mathbf{y}_{1}$ is the laminate direction, $\mathbf{y}_{2}$ is perpendicular to $\mathbf{y}_{1}$ in the tangential plane of the laminate, and $\mathbf{y}_{3}$ is perpendicular to the laminate and thus parallel to $\underline{\mathbf{r}}_{3}$. Thus, each layer will have a defined orientation given by a rotation around the base vector $\mathbf{y}_{3}$ with magnitude $\theta_{3}$.

To indicate the orientation of the different layers in a laminate we use a sequence of angles defined as in references $[66,69]$. For example, $[0,45,60,-30]$ means that the laminate is made of four layers oriented at 0, 45, 60 and -30 degrees. Subscripts are used to indicate the sequence of lamination, for example, $\left[0,45,60_{2},-30\right]=[0,45,60,60,-30]$ means that the layer oriented 60 degrees is repeated and, $[0,45]_{3}=[0,45,0,45,0,45]$ indicates that the sequence of layers oriented 0 and 45 degrees is repeated three times. Finally, the subscript $s$ indicates symmetry, thus $[0,45]_{s}=[0,45,45,0]$.

For each blade section, the orientation of the different layers in a laminate will be given by the geometry. The $\mathbf{y}_{i}$ frame will be related to the reference coordinate system. Vector $\mathbf{y}_{1}$ will be parallel to $\underline{\mathbf{B}}_{1}$, which is perpendicular to the blade section. As $\underline{\mathbf{y}}_{2}$ is tangent to the laminate, it will define an angle $\theta_{1}$ with vector $\underline{\mathbf{B}}_{2}$.

The lamination angle $\theta_{3}$ will be a property of the material so the rotation of the constitutive tensor could be performed a priori when the laminate is defined. On the other hand, the lamination angle on the blade section will depend on where the laminate is applied. For example, in straight-walled sectors, as in the shear-webs, it will depend on the wall side. In curved sectors as the outer-skin of the blade section, the angle will vary with the section's curvature. According to this, we will perform the coordinates transformations in two steps: First, a rotation around $\underline{\mathbf{r}}_{3}$ with a magnitude $\theta_{3}$ to transform the components from $\underline{\mathbf{r}}_{i}$ to $\underline{\mathbf{y}}_{i}$. Second, a rotation around $\mathbf{y}_{i}$ with a magnitude $\theta_{1}$ to express the constitutive tensor in the $\underline{\mathbf{B}}_{i}$ coordinate system. Only after these operations, the stiffness matrix is computed according to equation 4.18 . Figure 4.3 shows the different coordinate systems oriented with the layers and the laminate. 


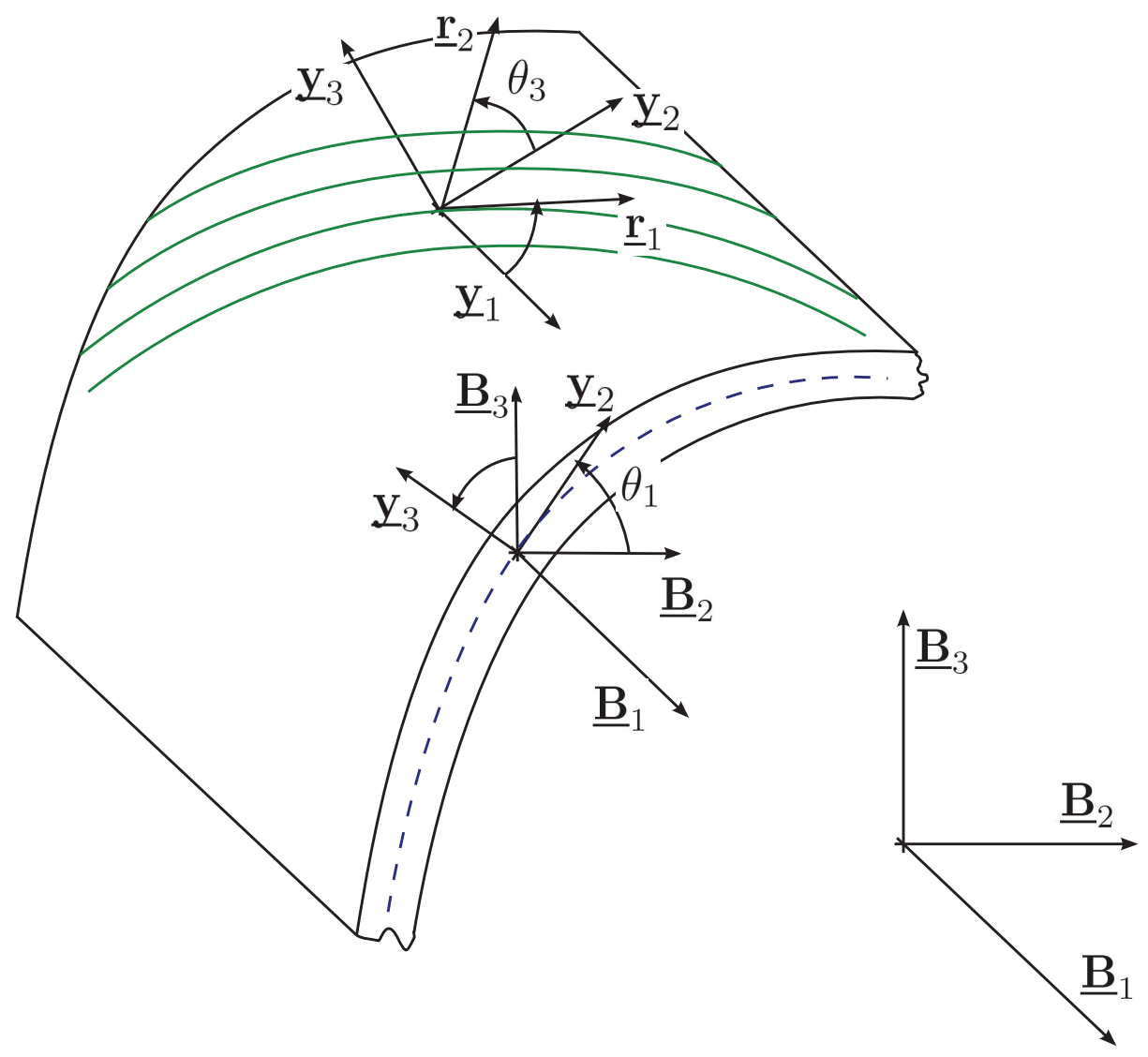

Figure 4.3. Reference frames involved in a laminate and their orientations. (Adapted from [12]) 


\section{NUMERICAL EXPERIMENTATION}

In this work we propose to use a set of rotor blades based on the 5-MW Reference Wind Turbine (RWT) project developed by NREL [16] as a test-bed for validation of the model and for the analysis of different aspects of blade aeroelastic dynamics that our model includes which are not covered in pervious models like FAST. Based on the REpower 5M wind turbine, the NREL's RWT was conceived for both onshore and offshore installations and it is well representative of state-of-the-art, utility-scale, multi-megawatt commercial wind turbines. Although full specific technical data is not available for the REpower $5 \mathrm{M}$ rotor blades, a baseline from a prototype blade was originally released by LM Glasfiber in 2001 for the Dutch Offshore Wind Energy Converter (DOWEC) 6MW wind turbine project [70,71] and later re-adapted by NREL. This suite of reports is considered one of the most detailed sources of information available for large wind turbines to date. In addition, the NREL 5-MW RWT project has been adopted as a reference model by the integrated European Union UpWind research program [72] and the International Energy Agency (IEA) Wind Annex XXIII Subtask 2 Offshore Code Comparison Collaboration (OC3) [73-75].

We will start this chapter describing the structural features of our blade as well as its general aerodynamic properties. Before getting into the details of the internal structure of the blade sections, we will review constructive blade aspects that will serve as the basis to understand the level of description required by our computational tools. Once this basis are set, we will present our blade internal structure, the finite element meshes associated to the structural computations, and will show how we match the structural response of the original blade in a series of validation tests. Then, we will analyze aspects of blade adaptiveness for the original blade and for two new configurations that we proposed, with forward and backward sweep. The latter tests will put in evidence new capacities of the Generalized Timoshenko model described in section 4.2, which allows us to capture the bending-twisting coupled modes in the fully populated $6 x 6$ blade section's stiffness matrix for the 1-D beam problem that we solve at each timestep in our aeroelastic simulations. This gives our model the capacity to simulate the dynamic performance of advanced adaptive blades unlike other codes. Finally, this chapter presents a series of tests on dynamic effects related to the inclusion of gravitational forces, which is also out of the scope of FAST. These tests will evince the potential of the full coupled aeroelastic model and how this tool 
could aid in studying fluid-structure interaction phenomena while exploring new horizons in rotor sizes and advanced blade prototypes.

To keep consistency with established practice in the different disciplines involved, in this chapter we are going to adopt the coordinate-system convention from complex-beam theory, where the $x$ axis is aligned with the span, to report all the variables concerning the behavior of the blade as a structural piece, indicating them with a subscript $r$. When the variables concern the behavior of the rotor as a whole, we are going to adopt the convention of the International Electrotechnical Commission (IEC) [36] for wind turbines, where the span is aligned with the $z$ axis (indicating them with a subscript $h$ ).

\subsection{Basic blade modelling and aerodynamic properties}

As stated for NREL's RWT project [16], the length of our rotor blade is set to be $61.5 \mathrm{~m}$. All basic aerodynamic properties as blade section chords, twist angles and basic spanwise stations distribution, also correspond to the original data in [16]. These aerodynamic properties, as well as the denomination of the basic airfoils at the design stations are included in table 5.1. Complementing the information in this table, figure 5.1 shows the blade section chords distribution along the span.



Figure 5.1. Chord distribution along the blade.

Airfoil types were also matched from the original blade. Stations 3 and 4 in table 5.1 are transitional airfoil shapes providing a smoother transition from the last cylindrical section, to the first thick airfoil in station 5. Two ellipsoidal shapes were used which match the chords originally given in [16]. Figure 5.2 shows the profiles of these two transitional shapes.

Back to the standard airfoils used for this rotor blade, figure 5.3a shows the profiles of the three thick airfoil sections corresponding to the inner regions of the blade, stations 5 to 8 , where a good structural support is required, while figure 5.3b shows the three airfoils used in the mid-span and tip regions where aerodynamic efficiency is the priority. As we can see, most of the basic airfoils used from the root to the mid span of this blade have the "DU" denomination. The DU airfoil series was specially developed for wind turbine applications by researchers at Delft University in Netherlands [76] and it is widely used by 
Table 5.1

Distributed blade aerodynamic properties.

\begin{tabular}{ccccl}
\hline Station & Location $[\mathrm{m}]$ & Twist angle $\left[^{\circ}\right]$ & Chord length $[\mathrm{m}]$ & Airfoil type \\
\hline 1 & 0 & 13.3080 & 3.5420 & Cylinder \\
2 & 1.3653 & 13.3080 & 3.5420 & Cylinder \\
3 & 4.1020 & 13.3080 & 3.8540 & Ellipsoid-1 \\
4 & 6.8327 & 13.3080 & 4.1670 & Ellipsoid-2 \\
5 & 10.2520 & 13.3080 & 4.5570 & DU 00-W-401 \\
6 & 14.3480 & 11.4800 & 4.6520 & DU 00-W-350 \\
7 & 18.4500 & 10.1620 & 4.4580 & DU 00-W-350 \\
8 & 22.5521 & 9.0110 & 4.2490 & DU 97-W-300 \\
9 & 26.6480 & 7.7950 & 4.0070 & DU 91-W-250 \\
10 & 30.7500 & 6.5440 & 3.7480 & DU 91-W-250 \\
11 & 34.8520 & 5.3610 & 3.5020 & DU 93-W-210 \\
12 & 38.9479 & 4.1880 & 3.2560 & DU 93-W-210 \\
13 & 43.0500 & 3.1250 & 3.0100 & NACA 64-618 \\
14 & 47.1521 & 2.3190 & 2.7640 & NACA 64-618 \\
15 & 51.2480 & 1.5260 & 2.5180 & NACA 64-618 \\
16 & 54.6673 & 0.8630 & 2.3130 & NACA 64-618 \\
17 & 57.3980 & 0.3700 & 2.0860 & NACA 64-618 \\
18 & 60.1347 & 0.1060 & 1.4190 & NACA 64-618 \\
19 & 60.5898 & 0.0903 & 1.1395 & NACA 64-618 \\
20 & 61.0449 & 0.0783 & 0.7787 & NACA 64-618 \\
\hline
\end{tabular}






(a)

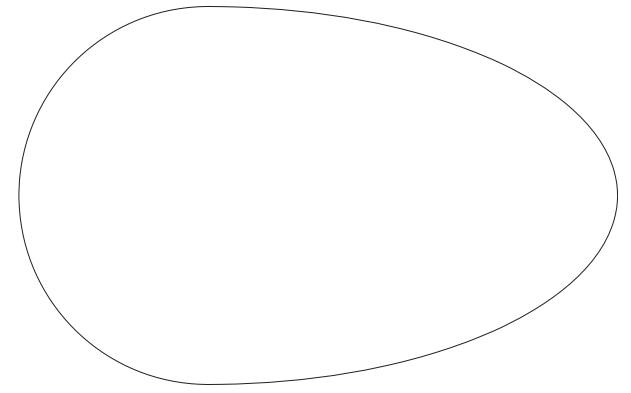

(b)

Figure 5.2. Profiles of ellipsoidal sections corresponding to: (a) station 3, and (b) station 4 on the blade span.
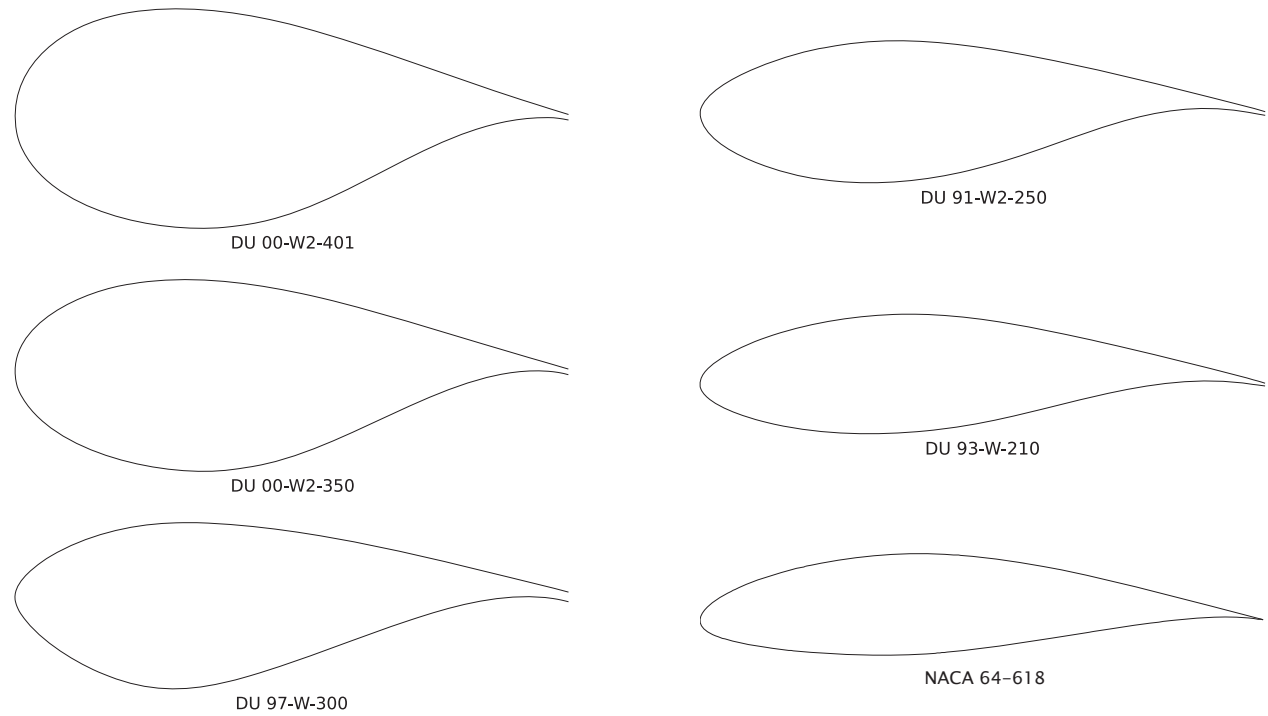

(a)

(b)

Figure 5.3. Profiles of the airfoil sections used to define the geometry of the blade. From top to bottom: (a) thick airfoils used in the inner regions, from station 5 to 8 of the blade-span, and (b) airfoils used in the mid-span and tip regions of the blade-span.

the wind-turbine industry.

Aerodynamic coefficients for the airfoils in table 5.1 were taken from [77] and [71]. Data for the cylinder, as well as the ellipsoidal shapes only include drag, but no lift coefficients. Cylinder and Ellipsoid-1 have a drag coefficient of 0.50. For Ellipsoid-2, the drag coefficient is lowered to 0.35 as its shape morphes from a bluff body towards a streamlined profile. Figure 5.4 shows lift, drags and momentum coefficients for the other six basic air- 


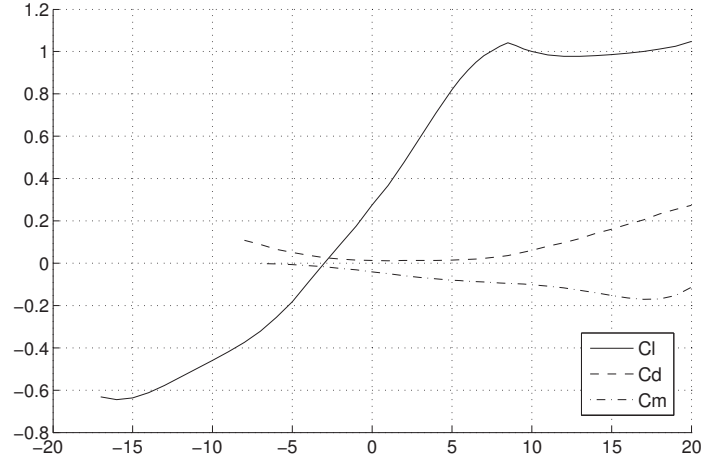

(a)

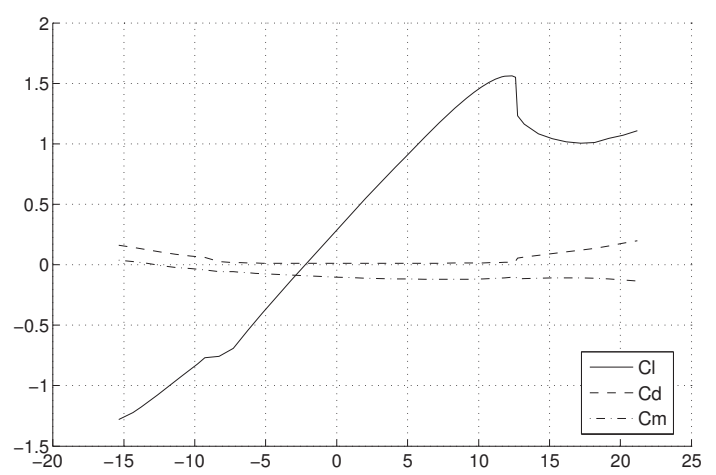

(c)

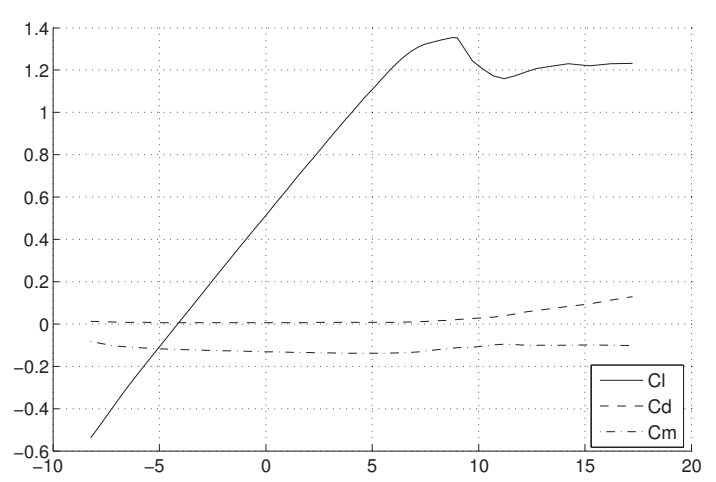

(e)



(b)



(d)

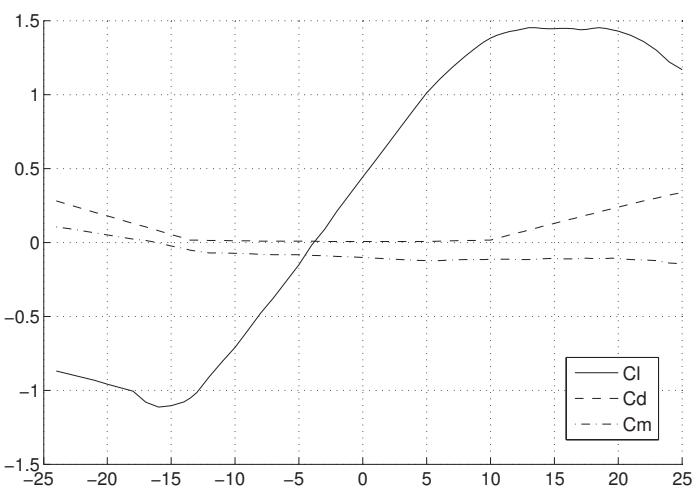

(f)

Figure 5.4. Lift, drag and momentum aerodynamic coefficients for airfoils: (a) DU 00-W-401, (b) DU 00-W-350, (c) DU 97-W-300, (d) DU 91-W-250, (e) DU 93-W-210, (f) NACA 64-618.

foils composing our rotor blade. This aerodynamic data is later processed using Viterna's method and corrected for rotational augmentation, as shown under section 3.3.1, for later use on the aeroelastic simulations. 


\subsubsection{Blade constructive aspects}

Wind turbine blade structure is a combination of two external aerodynamic shells, mounted on a box-beam spar which provides the main structural component to the aerodynamic forces. Analyzing a blade section (see figure 4.1) we can see the aerodynamic shells plus two spar caps which, together with two shear webs, form the box-beam spar. Constructive characteristics as thickness as well as number and orientation of fiberglass layers for the different structural components of the blade sections are covered in detail in reports published by SANDIA National Labs. [57,78]. According to these reports, the aerodynamic shells are mainly composed of $\pm 45^{\circ}$ layers, plus a small amount of randomly oriented fibers, gelcoat and filling resin. Shear webs, the the box-beam lateral walls, are made up of $\pm 45^{\circ}$ layers with a balsa wood core which provides the needed buckling resistance. Shear webs are usually located at the $15 \%$ and $45 \%$ of the airfoil's chord but, for sections closer to the blade's root, the positions are modified in order to increase the section's stiffness. Focusing now on the spar caps, these are made of $0^{\circ}$ layers and are the most important structural element as they give support to the bending loads on the blade. Finally, the blade sections has a reinforcement at its rear part, i.e. the trailing edge spline, also made up of $0^{\circ}$ oriented fibers which supports the bending loads in the chord-wise direction. Reports $[57,78]$ also provide a comprehensive description of lamination sequences and material properties.

We will now describe the thicknesses of the different layers on an airfoil section, according to the data from table 5.2, taken from [78], as well as the modeling for the blade sections proposed. The exterior of the airfoil section is covered by a $0,13 \mathrm{~mm}$ thick layer of Gelcoat, a resin-based paint which provides the adequate surface finishing as well as ultraviolet rays protection to the interior layers. following Gelcoat, we find a randomly oriented glass fibers layer to provide adherence between the subsequent layers with the top finishing one. This laminate is known as CSM (continuous strand mat) and its thickness is $0,38 \mathrm{~mm}$. After the $C S M$ layer, we find a preponderant orthotropic laminate with $\pm 45^{\circ}$ oriented fibers called $D B M$ (double bias mat). This laminate has a thickness given by $(A)=3,05 \times 10^{-5} \mathrm{~L}$ were $L$ is the length of the blade, as defined in table 5.2. These three layers remain constant all along the section contour.

The spar cap is made of three layers. The innermost and outermost are $2 / 3(A)$ thick internal reinforcements of $D B M$ going from the leading edge up to the rear shear web, that is to say $45 \%$ of the airfoil's chord. The mid layer provides the structural properties to the spar cap and it is made of an uniaxial laminate called $U N I$, where the fibers are oriented perpendicularly to the cross-section. The thickness of this layer was computed according to the guidance provided by [78] interpolating for our blade length. This mid layer is set between the two shear webs, say from $15 \%$ to $45 \%$ of the airfoil's chord. Thus, the foremost region of the airfoil section, from leading edge to $15 \%$ of the chord, will have besides the three external layers, the two corresponding to the spar cap reinforcements. After the $D B M$ 
Table 5.2

Summary of structural details, adapted from [78].

\begin{tabular}{|c|c|c|c|c|c|c|}
\hline $\begin{array}{l}\text { Item } \\
\text { ID }\end{array}$ & $\begin{array}{l}\text { Material De- } \\
\text { scription }\end{array}$ & $\begin{array}{l}\text { Placement } \\
\text { tion }\end{array}$ & $\begin{array}{l}\text { Tensile } \\
\text { Modulus } \\
\text { [Msi] }\end{array}$ & $\begin{array}{l}\text { Layer } \\
\text { Width } \\
\text { (\% of } \\
\text { chord) }\end{array}$ & $\begin{array}{l}\text { Layer } \\
\text { Thickness } \\
\text { [in] }\end{array}$ & $\begin{array}{l}\text { Placement Be- } \\
\text { hind L.E. (\% } \\
\text { of chord) }\end{array}$ \\
\hline 1 & Gelcoat & Outer Skin & 0.50 & $100 \%$ & 0.005 & $0 \%$ \\
\hline 2 & $3 / 4$ oz CSM & Outer Skin & 1.10 & $100 \%$ & 0.015 & $0 \%$ \\
\hline 3 & $\mathrm{DBM}$ & Outer Skin & 1.39 & $100 \%$ & (A) & $0 \%$ \\
\hline 4 & $\mathrm{DBM}$ & Spar Cap Reinf. & 1.39 & $45 \%$ & $2 / 3$ of $(\mathrm{A})$ & $0 \%$ \\
\hline 5 & C260/520Uni & Spar Cap (at Max) & 5.41 & $30 \%$ & (B) & $15 \%$ \\
\hline 6 & $\mathrm{DBM}$ & Spar Cap Reinf. & 1.39 & $45 \%$ & $2 / 3$ of $(A)$ & $0 \%$ \\
\hline 7 & Balsa & Aft Panel & 0.02 & $45 \%$ & $1 \%$ & $45 \%$ \\
\hline 8 & DBM & Inner Skin & 1.39 & $100 \%$ & (A) & $0 \%$ \\
\hline 9 & $\begin{array}{l}\text { Excess } \\
\text { Resin }\end{array}$ & Inside Inner Skin & 0.50 & $100 \%$ & 0.030 & $0 \%$ \\
\hline 10 & Balsa & $35 \%$ Web Core & 0.02 & See note & $\begin{array}{l}3 \% \text { of air- } \\
\text { foil }\end{array}$ & $35 \%$ \\
\hline 11 & DBM & $35 \%$ Web Skin & 1.39 & See note & See note & $35 \%$ \\
\hline 12 & C260/520Uni & T/E spline@ @5\% & 5.41 & $6 \%$ & (C) & $92 \%$ \\
\hline 13 & TE Plexus & To 2" fwd of TE & 0.05 & $2 \%$ & fill gap & $98 \%$ \\
\hline
\end{tabular}

Notes

(A) Thousandths $=1.2 \cdot$ bladelength $50 \mathrm{~m} \rightarrow .060 "$

(B) This value is found by converging to the required flapwise moment at $3,750 \mu$

(the spar cap is twice as thick at its center as at its edges)

(C) This value is found by converging to the required edgewise moment at $1,250 \mu$

For $15 \%$ Station:

The spar cap width is $60 \%$ of chord

The spar cap begins at $5 \%$ of chord

The spar cap reinforcement ends at $65 \%$ chord

For Shear Web:

The balsa thickness was $3 \%$ of max airfoil thickness

The height was half the section height (so each half reaches center)

Skins (thousandths) $=2 \cdot$ bladelength $50 m \rightarrow 0.100$ " each skin 
Table 5.3

Summary of Baseline Blade Material Properties, adapted from [57].

\begin{tabular}{lccccccc}
\hline Property & A260 & CDB340 & Spar Cap Mixture & Random Mat & Balsa & Gel Coat & Fill Epoxy \\
\hline$E_{x}[\mathrm{GPa}]$ & 31.0 & 24.2 & 25.0 & 9.65 & 2.07 & 3.44 & 2.76 \\
$E_{y}[\mathrm{GPa}]$ & 7.59 & 8.97 & 9.23 & 9.65 & 2.07 & 3.44 & 2.76 \\
$G_{x y}[\mathrm{GPa}]$ & 3.52 & 4.97 & 5.00 & 3.86 & 0.14 & 1.38 & 1.10 \\
$\nu_{x y}$ & 0.31 & 0.39 & 0.35 & 0.30 & 0.22 & 0.30 & 0.30 \\
$\nu_{f}$ & 0.40 & 0.40 & 0.40 & - & N/A & N/A & N/A \\
$w_{f}$ & 0.61 & 0.61 & 0.61 & - & N/A & N/A & N/A \\
$\rho[\mathrm{g} / \mathrm{cm} 3]$ & 1.75 & 1.75 & 1.75 & 1.67 & $0 . l 44$ & 1.23 & 1.15 \\
\hline
\end{tabular}

layers, and out of the spar cap zone, we find a balsa wood or solid foam layer providing laminar buckling stiffness to the airfoil contour. With a thickness that is $1 \%$ of the airfoil chord's, this layer goes from the leading edge to $15 \%$ of the chord, and from $45 \%$ of it up to the trailing edge. Finally, to provide a good finishing, the interior part of the airfoil is coated with an $(A)$ thick $D B M$ layer plus an extra $0.76 \mathrm{~mm}$ of resin.

Shear webs are made of a balsa wood or a solid foam core with a thickness equal to $3 \%$ of the airfoil thickness. This core is coated on each side with a $D B M$ layer $5 / 3(A)$ thick providing stiffness while the core separates the $D B M$ layers preventing laminar buckling.

Table 5.3, based on information from SANDIA report [57], shows basic properties of materials which are usually employed on wind turbine blade construction. In this work we used the given data for computing properties of the uniaxial laminate, balsa wood, gelcoat and epoxy resin. CSM and DBM are computed from UNI, combining this material with different fiber orientations in the right proportion.

\subsubsection{Blade structural properties}

According to the descriptions in section 5.1.1, material properties within the subregions corresponding to each of the blade section components were assumed homogeneous and equal to those of an equivalent material. The properties of this equivalent material, a $6 \times 6$ symmetric matrix with 21 independent coefficients, were computed by a weighted average of the actual laminate properties. Since the thicknesses of the region layers are very small compared to the actual size of the blade section, this assumption does not introduce significant errors. Besides, if more detail is required, our computational codes allow for independent meshing of every single layer of material separately using the exact properties.

After the internal regions and materials were defined, a triquadrilateral mesh was generated for a number of blade sections along the span. The preset master sections in table 5.1 
served as the basis for an innovative 3D-morphing technique based on variational cubicspline interpolation which allows us to obtain smooth transitions between the known $2 \mathrm{D}$ airfoil sections along the span of the blade. This way one can divide the blade into any number of sections larger than the known ones and generate finite element meshes for a more refined study of the structural features. As an example, figure 5.5 shows the profiles of two morphed airfoil sections located at the $20 \%$ and $60 \%$ of the blade span. Finite element meshes for this section and the master sections in table 5.1 are shown in figures 5.6 to 5.9 .



(a)

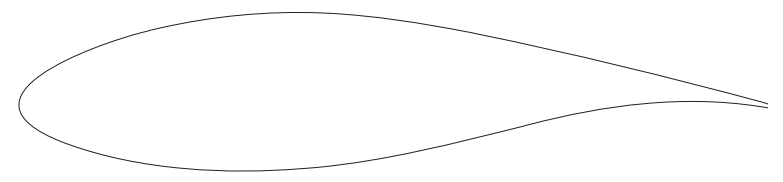

(b)

Figure 5.5. Profiles of morphed sections corresponding to: (a) $20 \%$ of the blade span, and (b) $60 \%$ of the blade span.

Using the described technique for the internal blade structure components, we refined 46 blade sections along the span to match the structural properties of the ones reported by NREL in [16]. The main targeted properties to refine were edgewise, flapwise, and torsional stiffness as well as mass density for every blade section. The pitch axis centring and the location of the aerodynamic coefficients reference points were computed according to information in [16] also. Table 5.4 summarizes this data for the refined sections defining two parameters: RefLinPos, the fraction of the chord from the leading edge to the reference line (the longitudinal axis) of the blade, which in a rectilinear blade like this coincides with the pitch axis, and AeroCentPos representing the fraction of chord from the leading edge to the aerodynamic center of each section. In the same table, Location is the position of the blade section along the blade span in meters, and Span is the corresponding bladelength fraction. Figure 5.10 shows the blade layout resulting from table 5.4, as well as the positions of the shear webs forming the blade's internal box-beam spar, as defined in section 5.1.1. 
Table 5.4

Blade section structural centering and aerodynamic coefficient reference point.

\begin{tabular}{lrll}
\hline $\begin{array}{l}\text { Location } \\
\text { [m] }\end{array}$ & & & \\
\hline 0.00 & 0.000 & 0.500 & 0.500 \\
0.20 & 0.003 & 0.500 & 0.500 \\
1.20 & 0.020 & 0.500 & 0.499 \\
2.20 & 0.036 & 0.495 & 0.490 \\
3.20 & 0.052 & 0.483 & 0.466 \\
4.20 & 0.068 & 0.471 & 0.441 \\
5.20 & 0.085 & 0.458 & 0.417 \\
6.20 & 0.101 & 0.446 & 0.392 \\
7.20 & 0.117 & 0.434 & 0.368 \\
8.20 & 0.133 & 0.422 & 0.343 \\
9.20 & 0.150 & 0.409 & 0.319 \\
10.20 & 0.166 & 0.397 & 0.294 \\
11.20 & 0.182 & 0.385 & 0.270 \\
12.20 & 0.198 & 0.375 & 0.250 \\
13.20 & 0.215 & 0.375 & 0.250 \\
14.20 & 0.231 & 0.375 & 0.250 \\
15.20 & 0.247 & 0.375 & 0.250 \\
16.20 & 0.263 & 0.375 & 0.250 \\
18.20 & 0.296 & 0.375 & 0.250 \\
20.20 & 0.328 & 0.375 & 0.250 \\
22.20 & 0.361 & 0.375 & 0.250 \\
24.20 & 0.394 & 0.375 & 0.250 \\
26.20 & 0.426 & 0.375 & 0.250 \\
28.20 & 0.459 & 0.375 & 0.250 \\
30.20 & 0.491 & 0.375 & 0.250 \\
32.20 & 0.524 & 0.375 & 0.250 \\
34.20 & 0.556 & 0.375 & 0.250 \\
36.20 & 0.589 & 0.375 & 0.250 \\
38.20 & 0.621 & 0.375 & 0.250 \\
40.20 & 0.654 & 0.375 & 0.250 \\
42.20 & 0.686 & 0.375 & 0.250 \\
44.20 & 0.719 & 0.375 & 0.250 \\
46.20 & 0.751 & 0.375 & 0.250 \\
48.20 & 0.784 & 0.375 & 0.250 \\
50.20 & 0.816 & 0.375 & 0.250 \\
52.20 & 0.849 & 0.375 & 0.250 \\
Continued 0 n next 039 &
\end{tabular}

Continued on next page... 
Table 5.4 - Continued

\begin{tabular}{llll}
\hline $\begin{array}{l}\text { Location Span } \\
\text { [m] }\end{array}$ & \multicolumn{2}{l}{ RefLinPos AeroCentPos } \\
\hline 54.20 & 0.881 & 0.375 & 0.250 \\
55.20 & 0.898 & 0.375 & 0.250 \\
56.20 & 0.914 & 0.375 & 0.250 \\
57.20 & 0.930 & 0.375 & 0.250 \\
57.70 & 0.938 & 0.375 & 0.250 \\
58.20 & 0.946 & 0.375 & 0.250 \\
58.70 & 0.954 & 0.375 & 0.250 \\
59.20 & 0.963 & 0.375 & 0.250 \\
59.70 & 0.971 & 0.375 & 0.250 \\
60.20 & 0.979 & 0.375 & 0.250 \\
60.70 & 0.987 & 0.375 & 0.250 \\
61.20 & 0.995 & 0.375 & 0.250 \\
61.50 & 1.000 & 0.375 & 0.250 \\
\hline
\end{tabular}

After the blade sections refinement, we generated a library of parameters with the optimal thicknesses for the different components of the internal structure. Combining this library with our 3D-morphing technique, we are now able to increase the number of intermediate blade sections at any region improving, if needed, the density of the 1-D mesh beyond the information originally reported in [16].

Table 5.5 summarizes the refined structural properties for the 46 blade sections. The first two columns are similar to those in table 5.4. BMassDen is the mass density of the blade expressed in $[\mathrm{kg} / \mathrm{m}]$. FlpStff, EdgStff and GLStff are respectively the flapwise, edgewise, and torsional stiffness for the blade section expressed in $\left[\mathrm{Nm}^{2}\right]$. Finally, flapwise, and edgewise blade section's inertia, FlpIner and EdgIner, are included, complementing the main structural properties. 
Table 5.5

Distributed blade structural properties.

\begin{tabular}{|c|c|c|c|c|c|c|c|}
\hline $\begin{array}{l}\text { Locati } \\
{[\mathrm{m}]}\end{array}$ & & $\begin{array}{l}\text { BMassDen } \\
{[\mathrm{kg} / \mathrm{m}]}\end{array}$ & $\begin{array}{l}\text { FlpStff } \\
{\left[N m^{2}\right]}\end{array}$ & $\begin{array}{l}\text { EdgStff } \\
{\left[N m^{2}\right]}\end{array}$ & $\begin{array}{l}\text { GLStff } \\
{\left[N m^{2}\right]}\end{array}$ & $\begin{array}{l}\text { FlpIner } \\
\text { [kgm] }\end{array}$ & $\begin{array}{l}\text { EdgIner } \\
{[\mathrm{kgm}]}\end{array}$ \\
\hline 0 & 000 & 32 & $1.800 \times 10^{10}$ & $1.800 \times 10^{10}$ & $\times 10^{9}$ & 65 & 1 \\
\hline 0.20 & 3 & & & $800 \times 10^{10}$ & $\times 10^{9}$ & & \\
\hline 1.20 & 0.020 & 40.007 & $1.960 \times 10^{10}$ & $1.960 \times 10^{10}$ & $5.810 \times 10^{9}$ & .93 & .93 \\
\hline 2.20 & 0.036 & 50.131 & $1.820 \times 10^{10}$ & $1.820 \times 10^{10}$ & $5.300 \times 10^{9}$ & .36 & 33.54 \\
\hline 3.20 & 0.052 & 78.927 & $1.540 \times 10^{10}$ & $1.980 \times 10^{10}$ & & & 7.44 \\
\hline 4.20 & 0.068 & 585.288 & $1.070 \times 10^{10}$ & $1.460 \times 10^{10}$ & $2.550 \times 10^{9}$ & 363 & 0.13 \\
\hline 5.20 & 0.085 & 27 & $7.330 \times 10^{9}$ & & & & 300 \\
\hline 6.20 & 0.101 & 431.718 & $6.420 \times 10^{9}$ & $9.260 \times$ & $1.820 \times$ & 39 & 836 \\
\hline 7.20 & 0.117 & 415.630 & $5.860 \times 10^{9}$ & $8.240 \times 10^{9}$ & $1.490 \times 10^{9}$ & 84 & 620.500 \\
\hline 8.20 & 0.133 & 40 & & & & & 006 \\
\hline 9.20 & 0.150 & 435. & & $6.860 \times 10^{9}$ & $6.160 \times$ & & 776 \\
\hline 10.20 & 0.166 & 487.452 & 9 & & & & 181 \\
\hline 11.20 & 0 . & 458. & & & & & \\
\hline 12.20 & 0.198 & 429.125 & $3.340 \times 10^{9}$ & $6.480 \times 10^{9}$ & $3.840 \times 10^{8}$ & 61 & 869 \\
\hline 13.20 & 0.215 & 406. & & & & & 523 \\
\hline 14.20 & 0.231 & 372. & & & & & 22 \\
\hline 15.20 & 0.247 & 372. & $\times 10^{9}$ & $5.240 \times 1$ & & & 817 \\
\hline 16.20 & 0.263 & & & & & & 703 \\
\hline 18.20 & 0.296 & 350.502 & $\times 10^{9}$ & $4.540 \times 10^{9}$ & $3.880 \times$ & & 744 \\
\hline 20.20 & 0.328 & 335 & & $4.220 \times 10^{9}$ & & 714 & .271 \\
\hline 22.20 & & 340.473 & & & & & 628 \\
\hline 24.20 & 0.394 & 355.369 & $\times 10^{9}$ & $3.730 \times 10^{9}$ & 1.120 & & .088 \\
\hline 26.20 & 0.426 & 338.738 & $\times 10^{8}$ & $3.400 \times 10^{9}$ & 8.960 & & 234.431 \\
\hline 28.20 & 0.459 & 310.922 & $8.220 \times 10^{8}$ & $3.070 \times 10^{9}$ & $9.920 \times 10^{7}$ & & 217.998 \\
\hline 30.20 & 0.491 & 284.503 & $\times 10^{8}$ & $2.720 \times 10^{9}$ & $1.080 \times 10^{8}$ & & 200.583 \\
\hline 32.20 & 0.524 & 263.924 & $5.310 \times 10^{8}$ & & & & 178.544 \\
\hline 34.20 & 0.556 & 269.853 & $4.030 \times 10^{8}$ & $2.310 \times 10^{9}$ & $7.480 \times 10^{7}$ & & 169.907 \\
\hline 36.20 & 0.589 & 233.829 & $\times 10^{8}$ & $1.830 \times 10^{9}$ & $6.530 \times 10^{7}$ & 19 & 138.727 \\
\hline 38.20 & 0.621 & 209.064 & $2.410 \times 10^{8}$ & $1.530 \times 10^{9}$ & $6.940 \times 10^{7}$ & 15.969 & 125.554 \\
\hline 40.20 & 0.654 & 190.141 & $1.760 \times 10^{8}$ & $1.320 \times 10^{9}$ & $6.610 \times 10^{7}$ & 12.398 & 115.712 \\
\hline 42.20 & 0.686 & 177.600 & $1.260 \times 10^{8}$ & $1.160 \times 10^{9}$ & & 8.842 & 99.078 \\
\hline 44.20 & 0.719 & 163.609 & $1.040 \times 10^{8}$ & $1.010 \times 10^{9}$ & $3.970 \times 10^{7}$ & 7.270 & 84.880 \\
\hline 46.20 & 0.751 & 147.221 & $9.100 \times 10^{7}$ & $7.970 \times 10^{8}$ & $3.040 \times 10^{7}$ & 6.181 & 65.809 \\
\hline 48.20 & 0.784 & 132.601 & & $6.910 \times 10^{8}$ & & 5.098 & 55.642 \\
\hline 50.20 & 0.816 & 113.774 & $6.100 \times 10^{7}$ & $5.180 \times 10^{8}$ & $1.830 \times 10^{7}$ & 4.071 & 41.930 \\
\hline
\end{tabular}

Continued on next page... 
Table 5.5 - Continued

\begin{tabular}{|c|c|c|c|c|c|c|c|}
\hline $\begin{array}{l}\text { Locati } \\
{[\mathrm{m}]}\end{array}$ & in & $\begin{array}{l}\text { BMassDen } \\
{[\mathrm{kg} / \mathrm{m}]}\end{array}$ & $\begin{array}{l}\text { FlpStff } \\
{\left[N m^{2}\right]}\end{array}$ & $\begin{array}{l}\text { EdgStff } \\
{\left[N m^{2}\right]}\end{array}$ & $\begin{array}{l}\text { GLStff } \\
{\left[N m^{2}\right]}\end{array}$ & $\begin{array}{l}\text { FlpIner } \\
\text { [kgm] }\end{array}$ & $\begin{array}{l}\text { EdgIner } \\
{[\mathrm{kgm}]}\end{array}$ \\
\hline 52.20 & 0.849 & 104.913 & $5.050 \times 10^{7}$ & $4.390 \times 10^{8}$ & $1.570 \times 10^{7}$ & 3.395 & 5.788 \\
\hline 54.20 & 0.881 & 95.769 & $3.910 \times 10^{7}$ & $4.020 \times 10^{8}$ & $1.400 \times 10^{7}$ & 2.713 & 32.817 \\
\hline 55.20 & 0.898 & 88.635 & $3.450 \times 10^{7}$ & $3.530 \times 10^{8}$ & $1.200 \times 10^{7}$ & 2.378 & 28.583 \\
\hline 56.20 & 0.914 & 80.062 & $3.020 \times 10^{7}$ & $2.990 \times 10^{8}$ & $9.500 \times 10^{6}$ & 2.043 & 23.813 \\
\hline 57.20 & 0.930 & 76.518 & $2.580 \times 10^{7}$ & $2.730 \times 10^{8}$ & $8.400 \times 10^{6}$ & 1.759 & 21.572 \\
\hline 57.70 & 0.938 & 70.728 & $2.360 \times 10^{7}$ & $2.470 \times 10^{8}$ & $6.240 \times 10^{6}$ & 1.551 & 18.559 \\
\hline 58.20 & 0.946 & 63.750 & $1.980 \times 10^{7}$ & $1.560 \times 10^{8}$ & $5.900 \times 10^{6}$ & 1.333 & 13.153 \\
\hline 58.70 & 0.954 & 58.966 & $1.540 \times 10^{7}$ & $1.380 \times 10^{8}$ & $5.400 \times 10^{6}$ & 1.072 & 11.849 \\
\hline 59.20 & 0.963 & 54.574 & $1.270 \times 10^{7}$ & $1.150 \times 10^{8}$ & $3.880 \times 10^{6}$ & 0.863 & 9.473 \\
\hline 59.70 & 0.971 & 52.417 & $9.820 \times 10^{6}$ & $1.010 \times 10^{8}$ & $2.970 \times 10^{6}$ & 0.665 & 8.034 \\
\hline 60.20 & 0.979 & 49.277 & $7.230 \times 10^{6}$ & $8.370 \times 10^{7}$ & $1.680 \times 10^{6}$ & 0.469 & 6.091 \\
\hline 60.70 & 0.987 & 41.763 & $2.460 \times 10^{6}$ & $3.100 \times 10^{7}$ & $1.610 \times 10^{6}$ & 0.209 & 3.100 \\
\hline
\end{tabular}

\subsubsection{Turbine specifications}

For the purpose of validation, the general specifications of the turbine also match the ones on NREL's report [16], which are summarized in table 5.6. Thus, the rotor has an upwind orientation and it is composed of three $61.5 \mathrm{~m}$ long blades. The hub diameter is $3 \mathrm{~m}$ and it is located at $90 \mathrm{~m}$ from ground level. Total rotor diameter is $126 \mathrm{~m}$. It has a precone of $2.5^{\circ}$ and an overhang distance of $5 \mathrm{~m}$ from the tower axis. The rated wind speed for this turbine is $11.4 \mathrm{~m} / \mathrm{s}$. 
Table 5.6

Global turbine parameters

\begin{tabular}{lr}
\hline Description & Value \\
\hline Rating & $5 \mathrm{MW}$ \\
Rotor Orientation & Upwind \\
Configuration & $3 \mathrm{blades}$ \\
Rotor, hub diameter & $126 \mathrm{~m}, 3 \mathrm{~m}$ \\
Hub Height & $90 \mathrm{~m}$ \\
Rated wind speed & $11.4 \mathrm{~m} / \mathrm{s}$ \\
Rated rotor speed & $12.1 \mathrm{rpm}$ \\
Overhang & $5 \mathrm{~m}$ \\
Rotor precone & $2.5^{\circ}$ \\
\hline
\end{tabular}




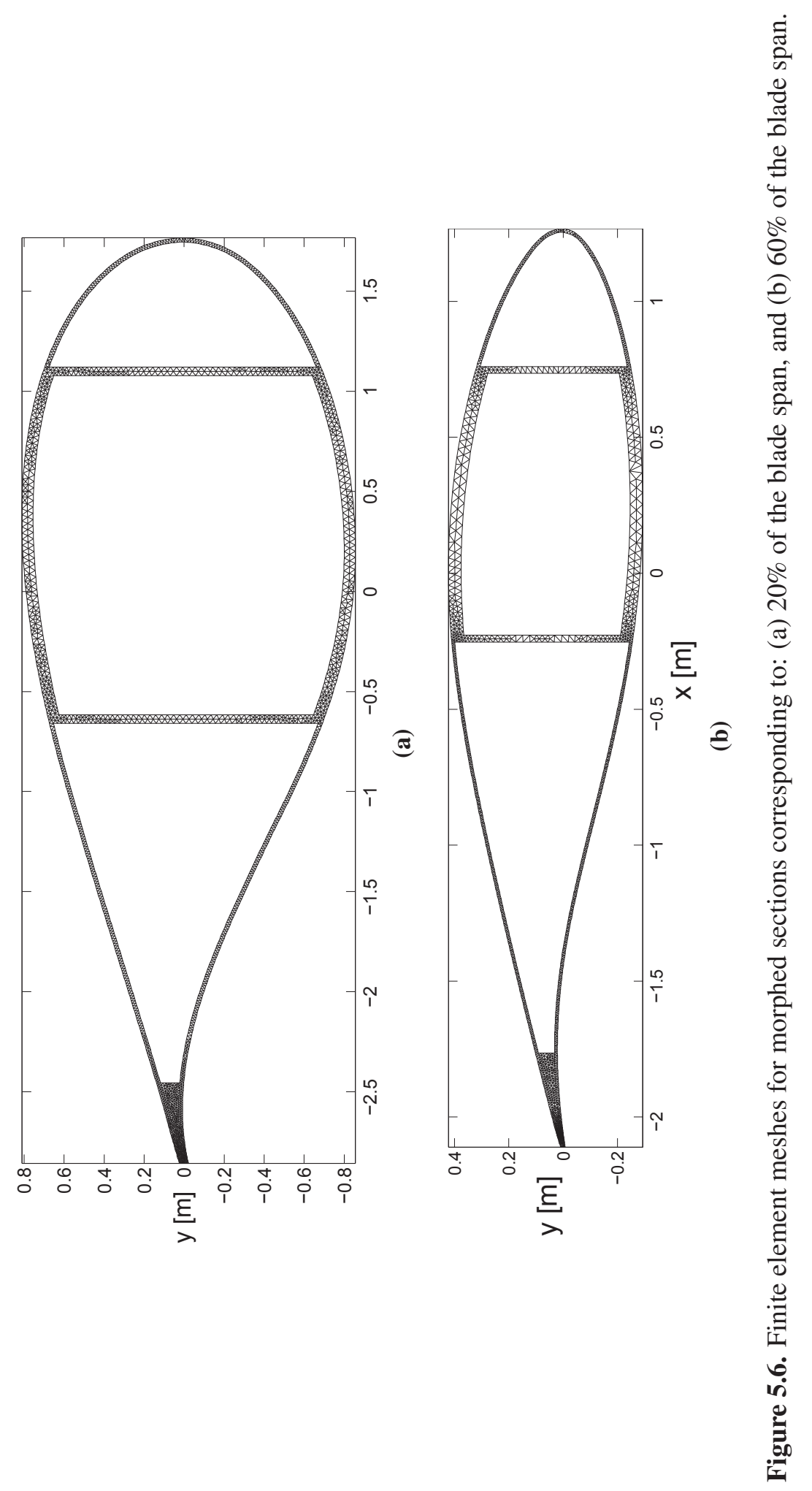




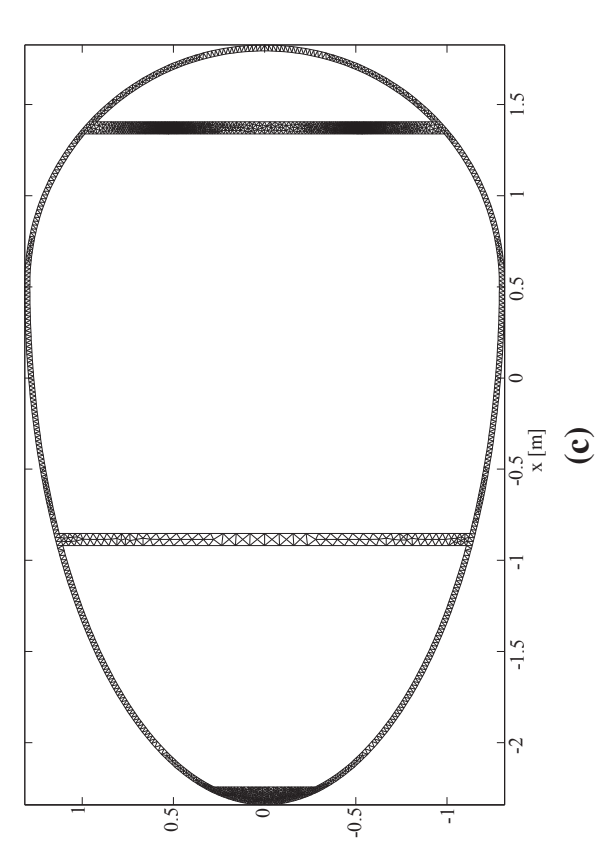

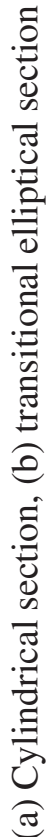

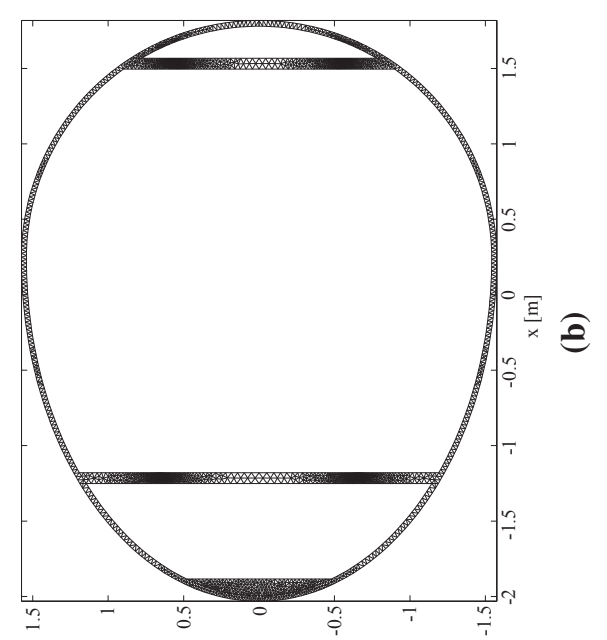

.0





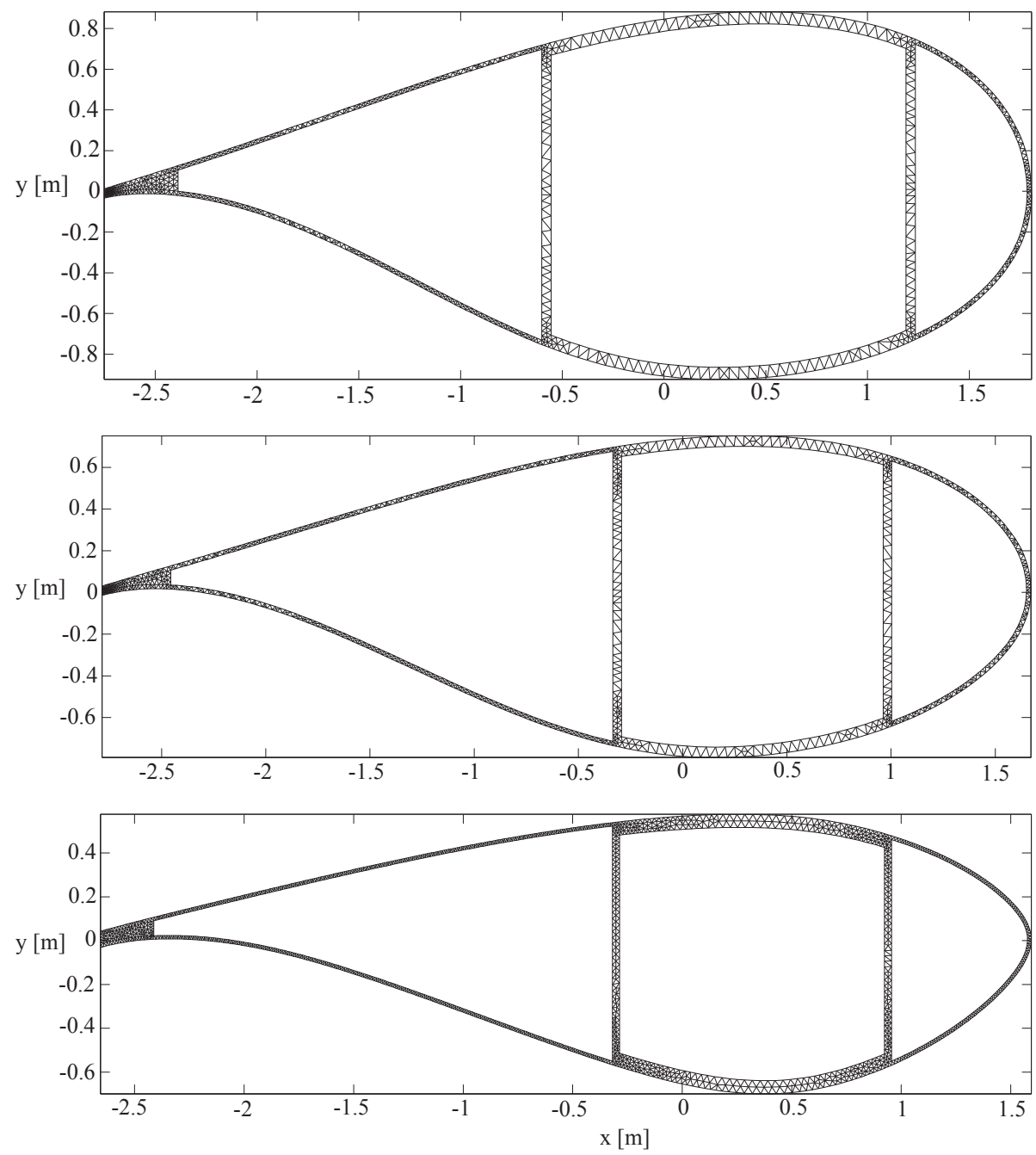

Figure 5.8. Finite element meshes for the master sections of the inner blade region. From top to bottom, DU 00-W-401, DU 00-W-350, DU 97-W-300. 

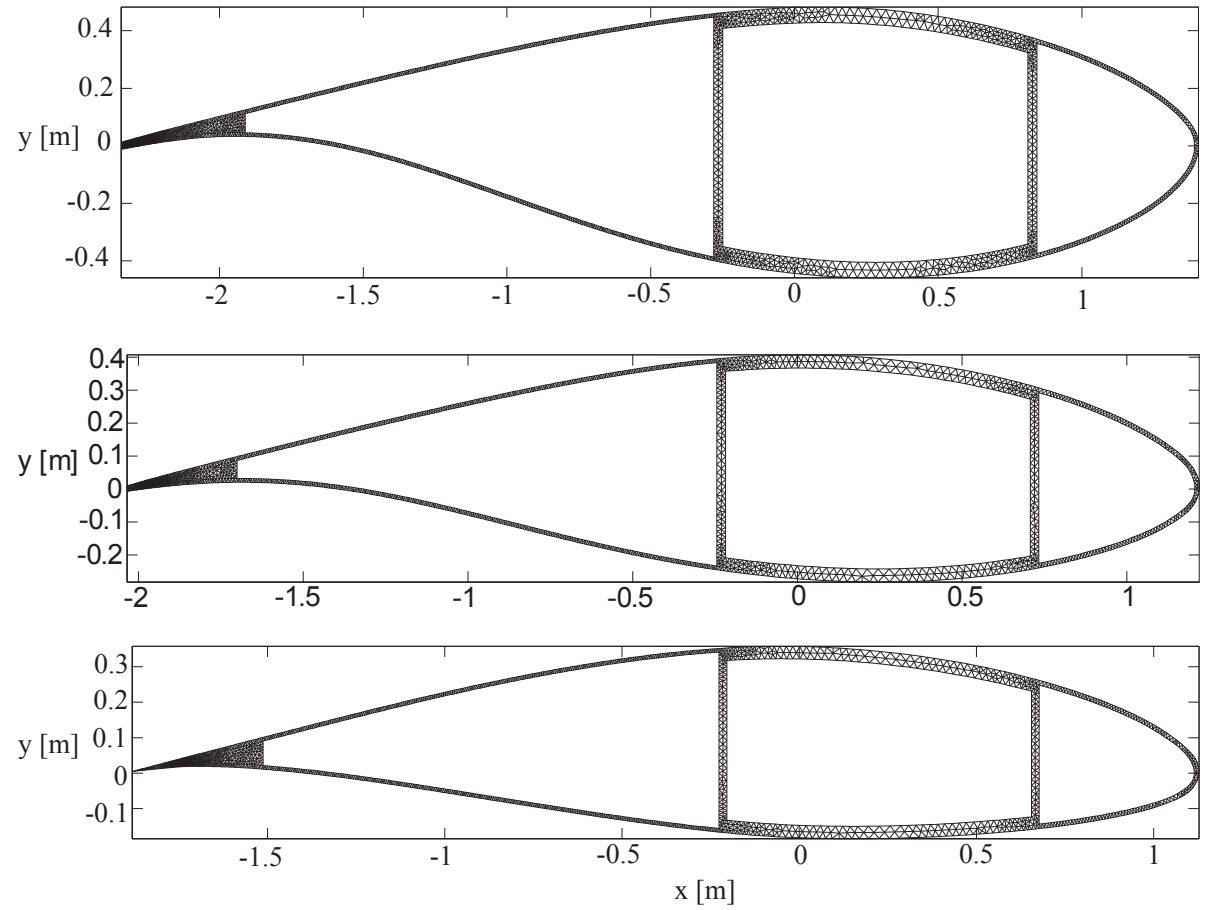

Figure 5.9. Finite element meshes for the master sections of the outer blade region. From top to bottom, DU 91-W-250, DU 93-W-210, NACA 64-618. 


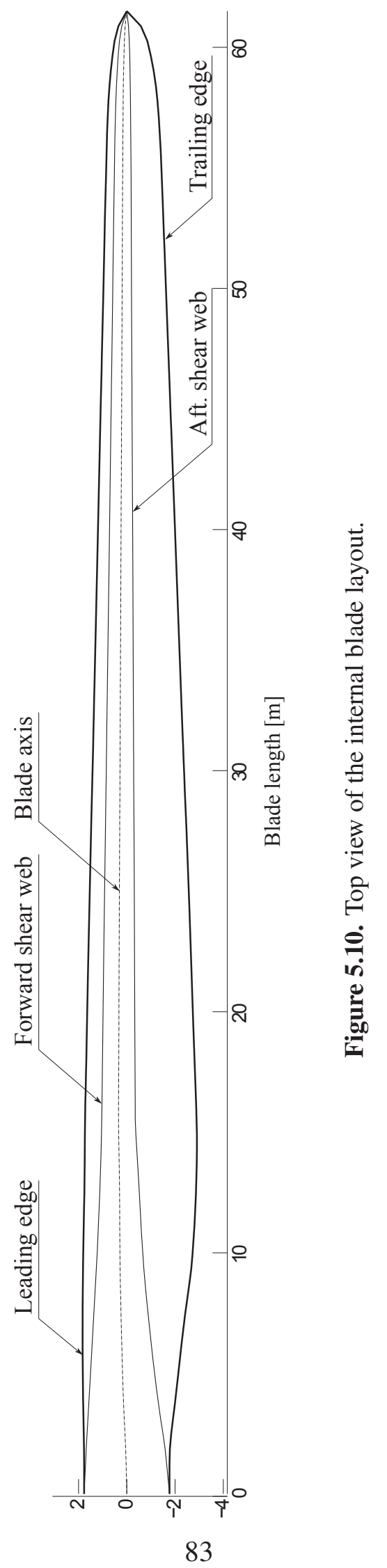




\subsection{Validation Tests}

In this section we will show a series of validation tests. We will start by presenting a steady-state solution around the nominal working condition, including the vibrational modes obtained from an eigenvalue analysis of a linearized solution around the steadystate. Next, we will test our model under an acceleration ramp simulating an arbitrary start-up from zero angular velocity to nominal operation conditions on the rotor. Finally, a computation of the blade-pitch angle for power-control over high wind speeds will be presented.

\subsubsection{Aeroelastic steady-state case}

After computing stiffness and inertia matrices for a discrete number of cross-sections along the span of the blade, we applied the dimensional reduction technique described in section 4.2. Tip speed ratio for the nominal operational condition is $\lambda=7$, so the tangential velocity at the tip of the blade is $80 \mathrm{~m} / \mathrm{s}$.

For this nominal working condition, the power output computed for our rotor is $5.455 \mathrm{MW}$ which, taking into account that, as in any BEM approach, the interference of the tower and the nacelle is computed only approximately, is in very good agreement with the reported power for the NREL-5MW reference turbine rated at 5.296MW according to $[16]$.

Figure 5.11 shows the displacement of the blade's reference-line (blade axis) $\mathbf{U}_{\mathbf{h}}$ when it is subjected to the aerodynamic steady load in normal operational conditions. Figure 5.12, shows the corresponding rotations of the blade sections $\boldsymbol{\theta}_{h}$. These geometrical magnitudes were referred to a coordinate system aligned with the rotor's plane, according to standards from the International Electrotechnical Commission (IEC) [36]. Hence, the first unit vector is normal to the rotor's plane (i.e. axial) pointing downwind, the second is in the rotor's tangential direction pointing to the blade's trailing edge, and the third unit vector is in the radial direction pointing to the blade tip.

From figure 5.11 we can see that the displacement $U_{h_{1}}$ of the blade's tip, normal to the rotor's plane, is $5.73 \mathrm{~m}$. This is perfectly consistent with results shown in [16]. Added to this, the tangential displacement $U_{h_{2}}$ is $0.78 \mathrm{~m}$ in the negative direction, meaning that aerodynamic forces are bending the blade in the direction towards its rotation as the rotor is producing a positive driving torque.

In figure 5.12, angles $\theta_{h_{2}}$ and $\theta_{h_{1}}$ are directly associated with blade bending in the normal and tangential directions to the rotor plane, that is $U_{h_{1}}$ and $U_{h_{2}}$ respectively. It is 

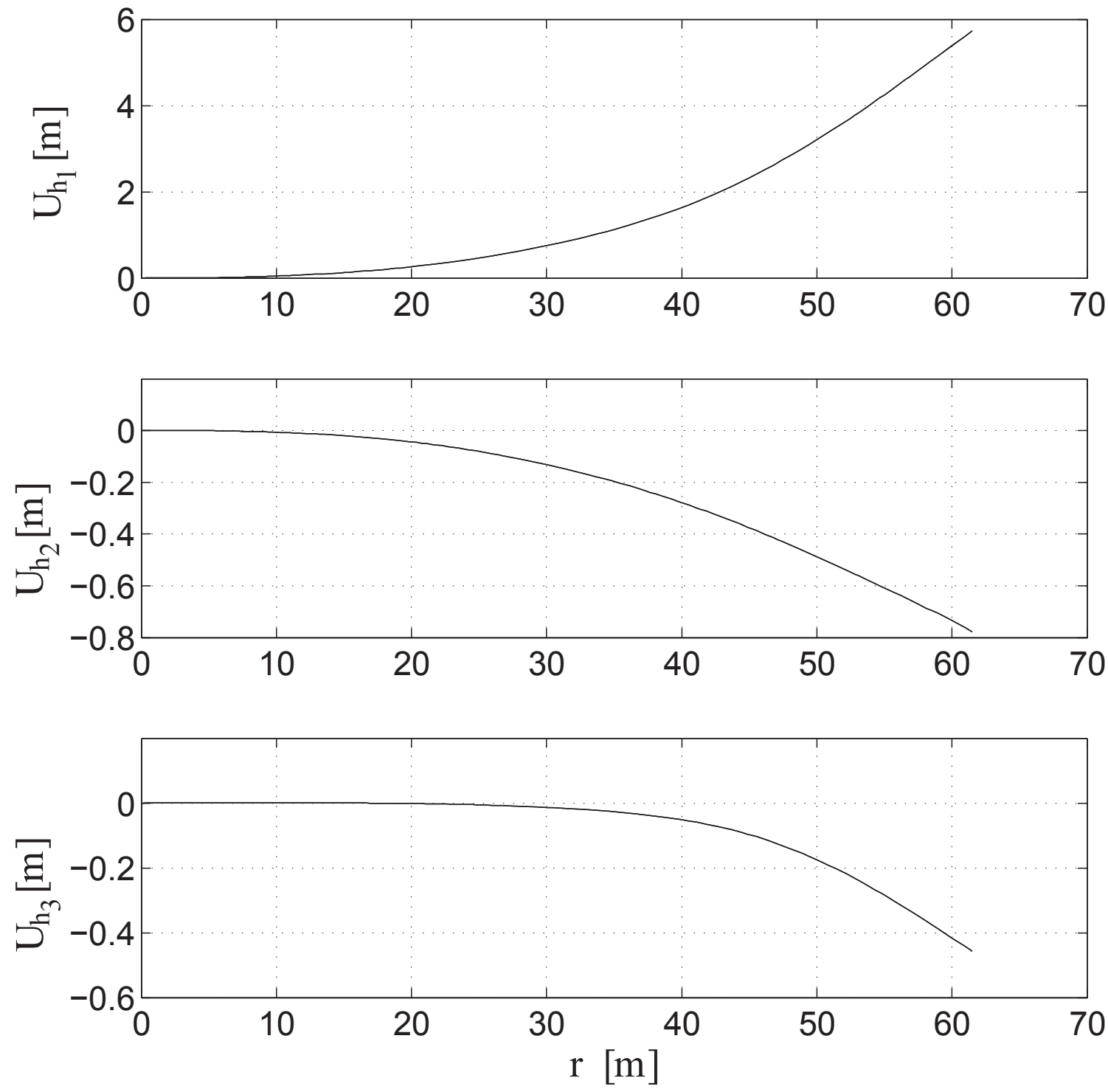

Figure 5.11. Linear displacements of the reference-line $\mathbf{U}_{\mathbf{h}}$ when the beam is subjected to a steady load in normal operational conditions (referred to a coordinate system aligned with the rotor's plane) 

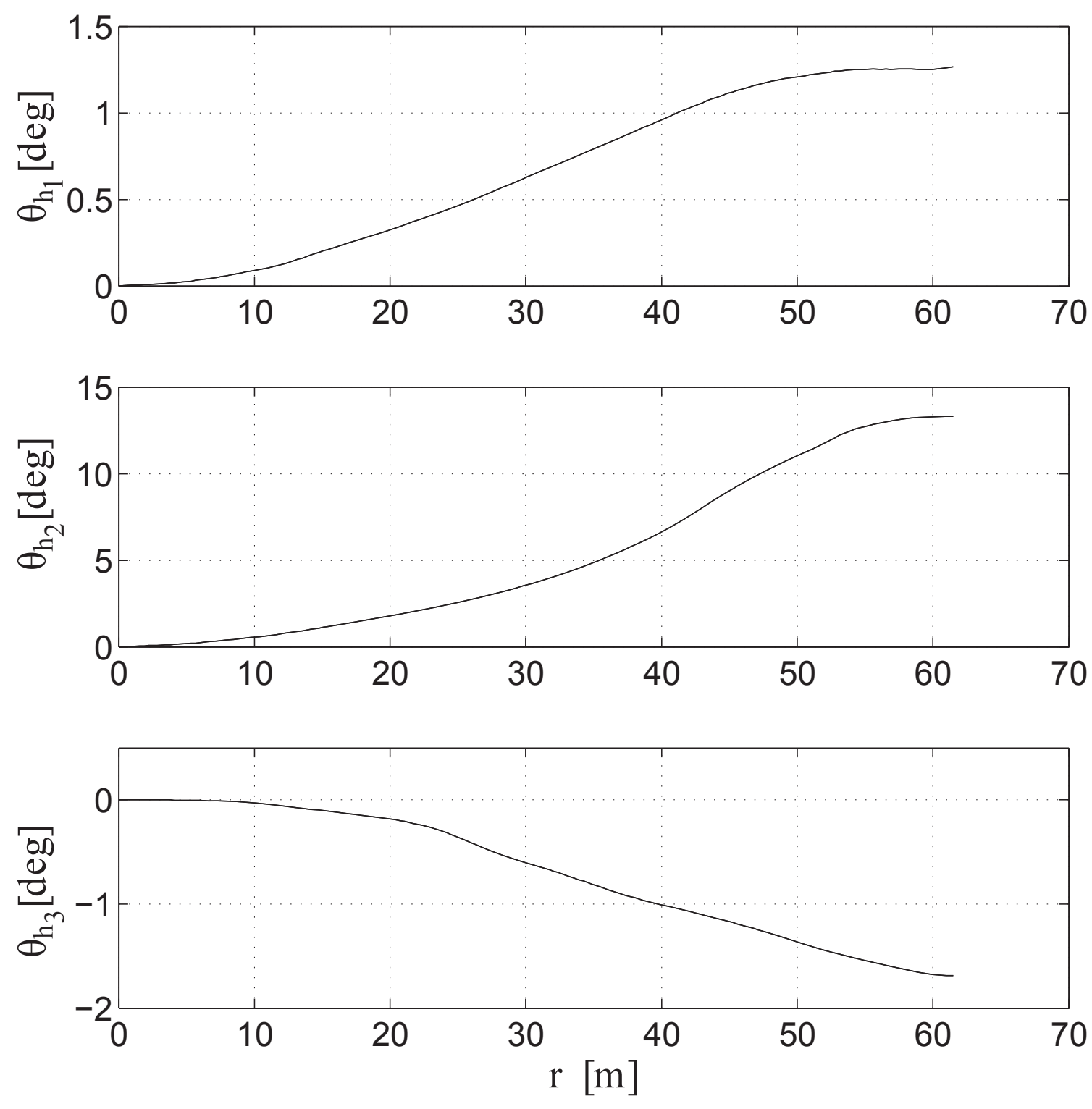

Figure 5.12. Rotations of the beam sections $\boldsymbol{\theta}_{h}$ when the beam is subjected to a steady load in normal operational conditions (referred to a coordinate system aligned with the rotor's plane). 

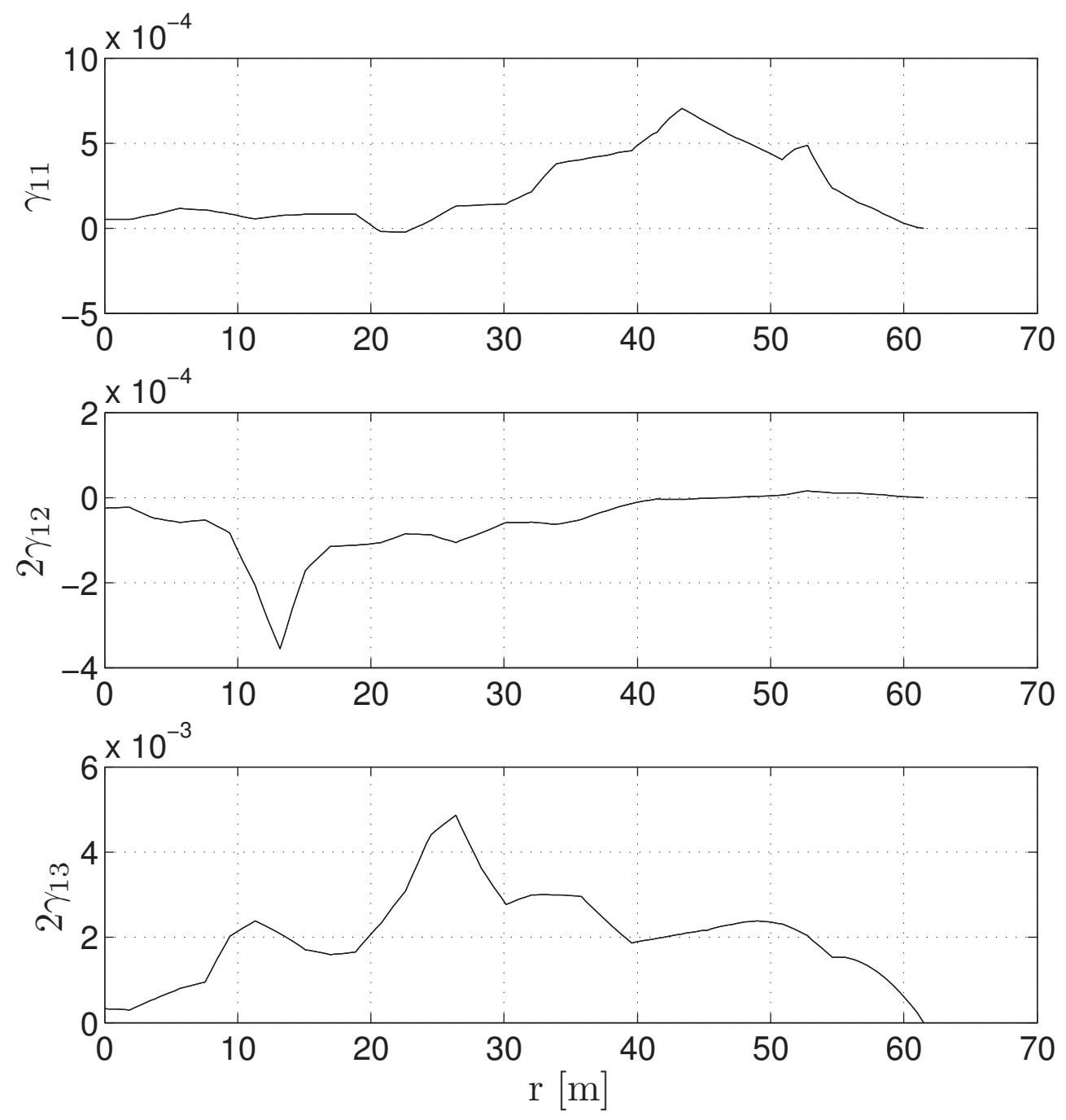

Figure 5.13. Timoshenko measures of deformation. From top to bottom: $\gamma_{11}$, associated to stretching. $2 \gamma_{12}$, associated to distortion. $2 \gamma_{13}$, associated to distortion.

important to note that angle $\theta_{h_{2}}$ represents the angular displacements which deforms the blade's axis out of the rotor's plane.

Figures 5.13 and 5.14 show the corresponding Timoshenko measures of deformation for the equivalent beam, as described in section 4.2. 

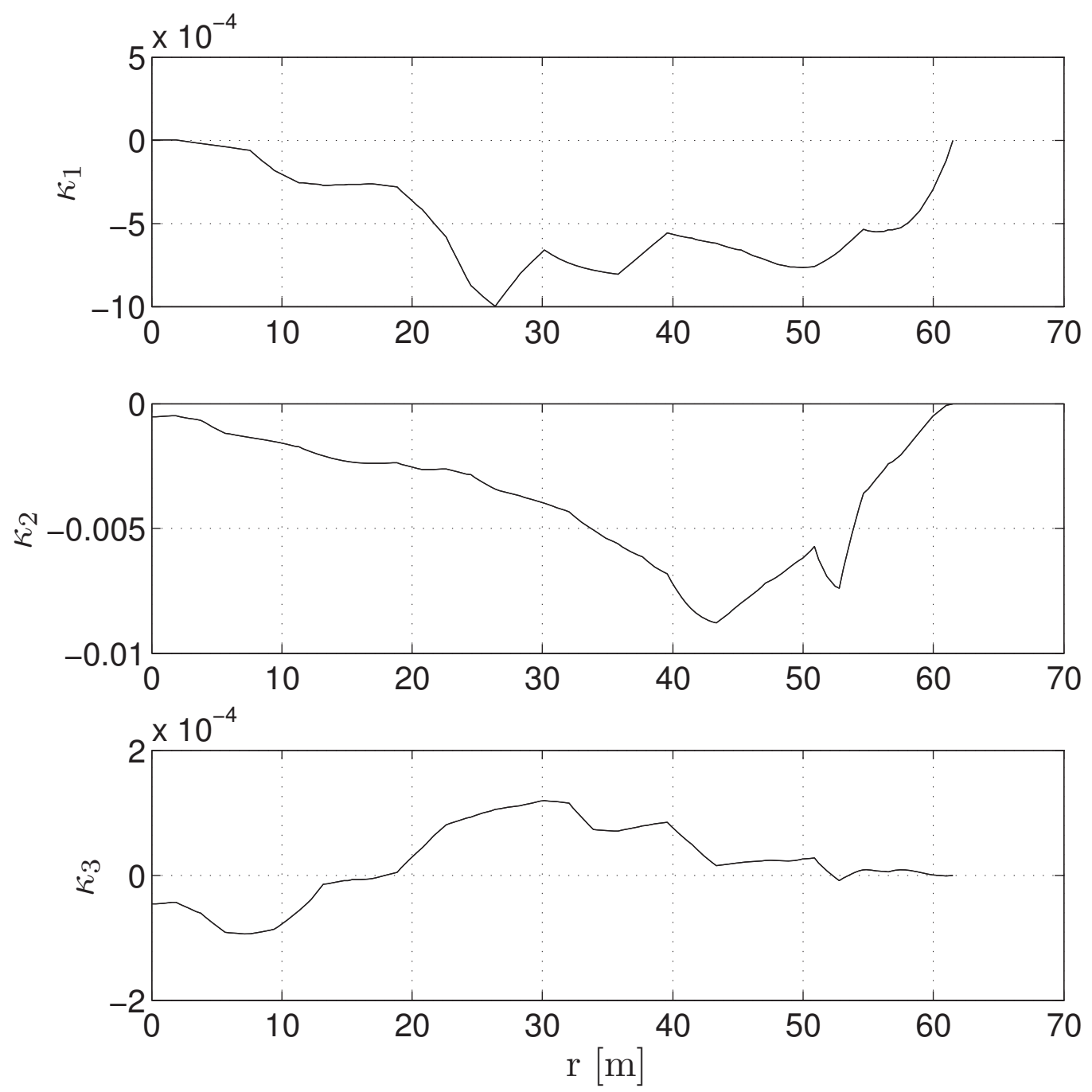

Figure 5.14. Timoshenko measures of deformation. From top to bottom: Torsion, $\kappa_{1}$. Curvature $\kappa_{2}$. Curvature $\kappa_{3}$. 
Table 5.7

List of frequencies and dominant components of $\mathbf{U}_{\mathbf{h}}$ and $\boldsymbol{\theta}_{h}$ for the first ten modes of vibration.

\begin{tabular}{cccc} 
Mode & frequency $[\mathrm{Hz}]$ & Dominant $U_{h}$ & Dominant $\theta_{h}$ \\
\hline 1 & 0.7066 & $U_{h_{1}}$ & $\theta_{h_{2}}$ \\
2 & 1.0188 & $U_{h_{2}}$ & $\theta_{h_{1}}$ \\
3 & 1.8175 & $U_{h_{1}}$ & $\theta_{h_{2}}$ \\
4 & 3.3403 & $U_{h_{2}}$ & $\theta_{h_{3}}$ \\
5 & 3.9493 & $U_{h_{1}}$ & $\theta_{h_{2}}$ \\
6 & 6.4682 & $U_{h_{2}}$ & $\theta_{h_{3}}$ \\
7 & 6.6851 & $U_{h_{1}}$ & $\theta_{h_{3}}$ \\
8 & 8.0129 & $U_{h_{2}}$ & $\theta_{h_{3}}$ \\
9 & 8.2403 & $U_{h_{1}}$ & $\theta_{h_{2}}$ \\
10 & 9.7819 & $U_{h_{1}}$ & $\theta_{h_{2}}$ \\
\hline
\end{tabular}

Table 5.8

Frequencies comparison for the first three modes according to NREL report [16].

\begin{tabular}{cccc} 
Mode & frequency $[\mathrm{Hz}]$ & FAST & ADAMS \\
\hline 1 & 0.7066 & 0.6993 & 0.7019 \\
2 & 1.0188 & 1.0793 & 1.0740 \\
3 & 1.8175 & 1.9223 & 1.8558 \\
\hline
\end{tabular}

\subsubsection{Vibrational modes around the aeroelastic steady-state}

We present now an analysis of the vibrational modes around the aeroelastic steady-state solution obtained in section 5.2.1. Vibrational modes are obtained from the solution of an eigenvalue problem as described in section 4.2. As the resulting eigenvalues are complex conjugate, their imaginary part represents frequencies while their non-zero real part corresponds to damping effects coming from non-conservative fields in the $1 \mathrm{D}$ functional. We will return to this concept later in section 5.3.1 where we consider the subject of blade adaptiveness.

Vibrational mode analysis provides relevant information about both the natural vibrational frequencies of the blade around a steady-state condition, and for the modes of deformation along the blade span. Table 5.7 summarizes the first 10 modes obtained showing the frequencies together with the corresponding dominant component for the displacements and the rotations of the blade section. Table 5.8 shows a comparison of the frequencies obtained for the first three modes with the values reported by NREL in [16] using FAST [56] and ADAMS [79] software. These values seem to be in good agreement, especially for the first mode, with a difference of only $1 \%$ between the three codes. 
Figures 5.15 and 5.16 show some of the deformation modes. Every mode shown includes displacements and rotations of the blade sections normalized by the value of the dominant component.

\subsubsection{Recovering of displacements, strains and stresses for the blade sections}

According to the technique described in appendix A, after computing the global deformations from the 1-D analysis for the equivalent beam, we recovered the corresponding 3-D fields (displacements, strains, and stresses) using the 3-D warping functions presented in section A.1. Knowing the stress state of the blade sections is of utmost importance in the analysis of rotor blades to improve the lifetime and reliability of the design. For the previously solved aeroelastic steady-state, we present the six components of the JaumannBiot-Cauchy stress tensor for a blade section located at the $40 \%$ of the span. This region is particularly interesting as it combines energy production and structurally supports significant stress accumulation compared to other regions along the blade span. Figures 5.17 and 5.18 show the six components of the stress tensor in the coordinates of the undeformed configuration $\left(X^{1}, X^{2}, X^{3}\right)$, as described in section 4.1. The dominant stress component, $\mathrm{Z}_{11}$, at the top of figure 5.17 is the one primarily associated with the out of rotor-plane bending loads. Note here how the lower spar-cap is subjected to tensile stress while the upper one is under compression stress.

\subsubsection{Dynamic simulation of rotor start-up}

After successful validations for aeroelastic steady-state conditions we will now test our computational tools under dynamic simulations. As described in section 2.1 the Common ODE Framework controls the interrelation of the different computational modules reacting to the behavior of the different physical phenomena. Here, the use of a nonlinear adaptive ODE algorithm provides the means to control the time-dependant solution for the dynamic aerolastic problem. The first dynamic simulation that we show in this section is a rotor start-up situation. The rotor start-up is modeled starting from a completely still condition (i.e. tip-speed ratio $\lambda=0$ ) to a full normal operational condition $(\lambda=7)$ varying only the rotational speed of the rotor from 0 to $12.1 \mathrm{rpm}$ (see table 5.6). For the purpose of testing the aeroelastic response of the blades in a generic star-up situation (regardless of any specific control strategy that eventually could be adopted) we assumed an acceleration ramp for the turbine rotational speed with a semi-Gaussian profile, which provides a smooth acceleration at the beginning and the end of the start-up cycle (see figure 5.19). The total start-up simulation time is set to be five minutes, which represents a typical start-up time of utility-scale commercial turbines. 

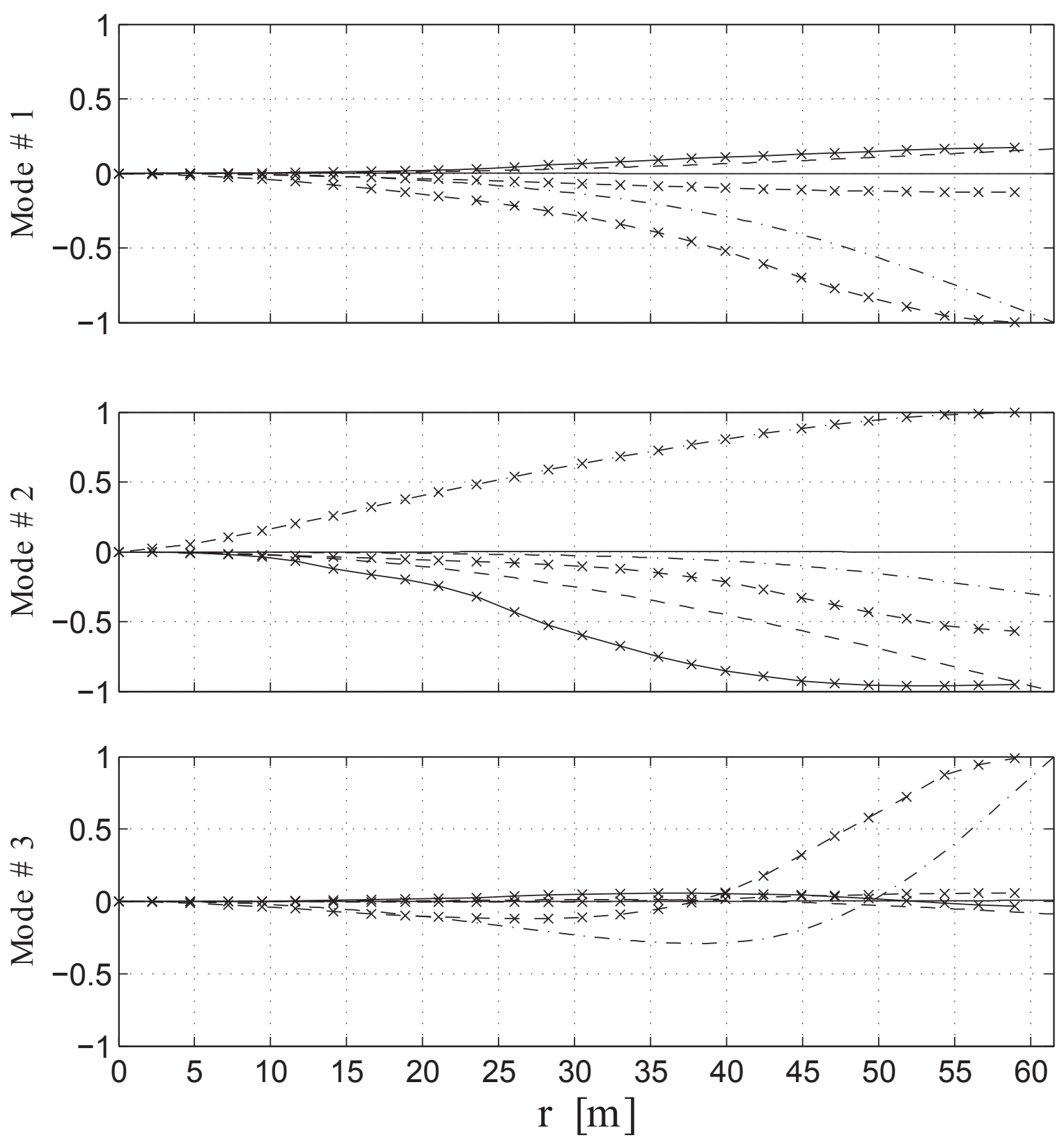

$$
-\cdot-\mathrm{U}_{\mathrm{h}_{1}}---\mathrm{U}_{\mathrm{h}_{2}}-\mathrm{U}_{\mathrm{h}_{3}} \cdot-\times-\cdot \theta_{\mathrm{h}_{1}}-*-\theta_{\mathrm{h}_{2}} \underset{*}{*} \theta_{\mathrm{h}_{3}}
$$

Figure 5.15. Amplitude of $\mathbf{U}_{\mathbf{h}}$ and $\boldsymbol{\theta}_{h}$ for three vibrational modes around the aeroelastic steadystate configuration (normalized by the dominant component). From top to bottom modes \# 1, 2 and 3. 

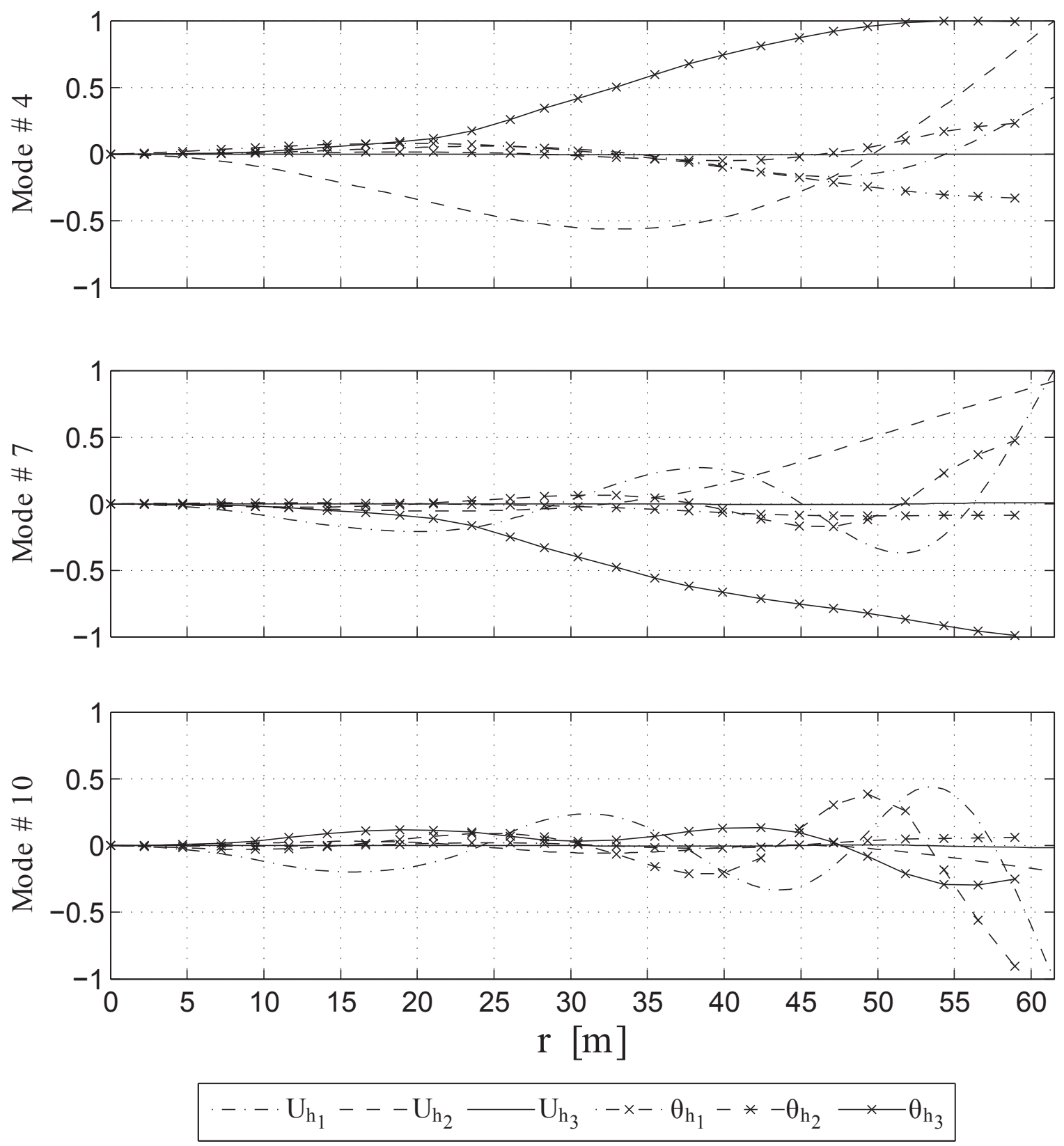

Figure 5.16. Amplitude of $\mathbf{U}_{\mathbf{h}}$ and $\boldsymbol{\theta}_{h}$ for three vibrational modes around the aeroelastic steadystate configuration (normalized by the dominant component). From top to bottom modes \# 4, 7 and 10. 



Figure 5.17. The six components of the Jaumann-Biot-Cauchy stress tensor $\mathbf{Z}=\mathcal{S} \boldsymbol{\Gamma}$ for the section located at $40 \%$ of the blade span (referred to the undeformed coordinate system $\left(X^{1}, X^{2}, X^{3}\right)$ in Pa). From top to bottom: $Z_{11}, Z_{12}$ and $Z_{13}$. 

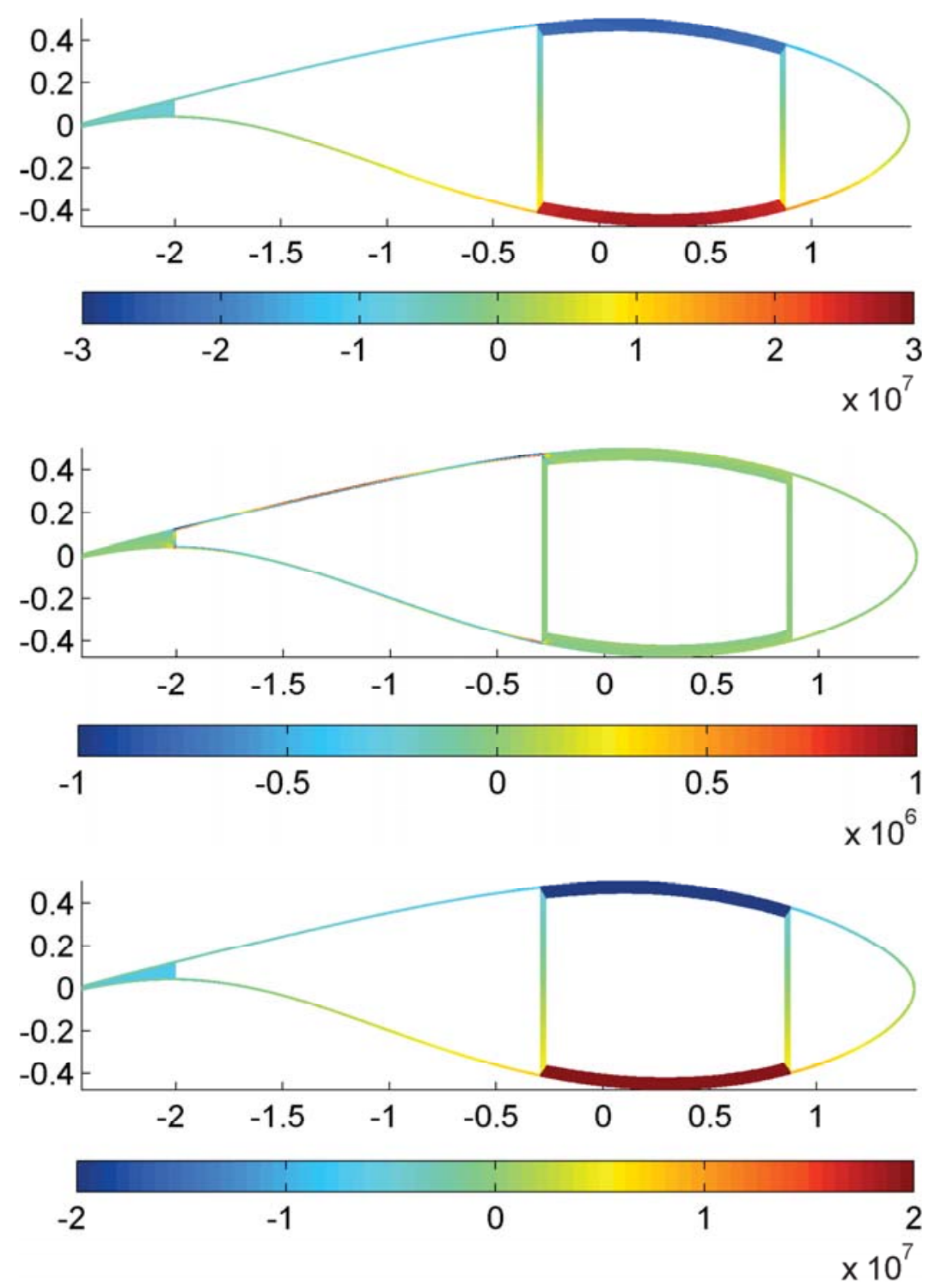

Figure 5.18. The second three components of the Jaumann-Biot-Cauchy stress tensor $\mathbf{Z}=\mathcal{S} \Gamma$ for the section located at $40 \%$ of the blade span (referred to the undeformed coordinate system $\left(X^{1}, X^{2}, X^{3}\right)$ in Pa). From top to bottom: $Z_{22}, Z_{23}$ and $Z_{33}$. 


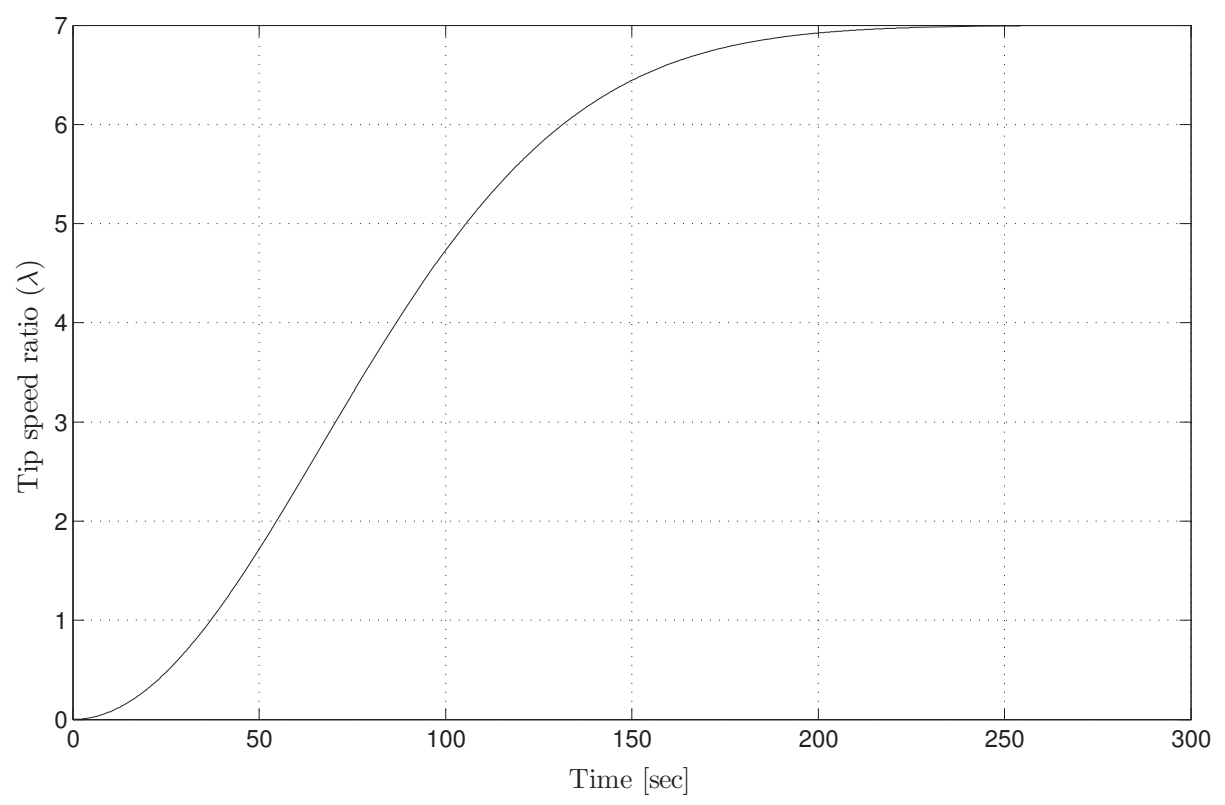

Figure 5.19. Acceleration ramp for a full start-up dynamic simulation. Variation of the rotational speed of the turbine from 0 to $12.1 \mathrm{rpm}$, at constant rated wind speed according to table 5.6.

Figures 5.20 and 5.21 show the time evolution of the displacements and rotations for the blade section located at the $95 \%$ of the span, a location where displacements could be better appreciated. Figure 5.22 shows the evolution of the chord-wise and chord-normal aerodynamic forces from the LSR-BEM model. It is interesting to note that when the turbine decelerates, reaching the nominal operational condition, small vibrations appear. These light vibrations could be a consequence of inertia effects on the blade structure, possibly coupled with aerodynamic effects (which seems to be confirmed by the corresponding fluctuations on the aerodynamic forces in figure 5.22 for the same time span. Finally, figures 5.23 and 5.24 show the time evolution of the rotor's torque and power output at the main shaft.

\subsubsection{Blade pitch control for power limitation at wind speeds above the rated.}

According to reference [16], this wind turbine blade is not supposed to be actuated for pitch control during operation at wind speeds lower than the rated. Blade pitch control is effective for wind speeds beyond $11.4 \mathrm{~m} / \mathrm{s}$ and, under these circumstances, the goal is to maintain a constant power output and rotor speed. To this end, we reproduced the tests reported in [16], which include computation of the Sensitivity of Aerodynamic Power to Blade Pitch using our model. Blade-pitch sensitivity is a rotor's aerodynamic property which depends on the wind speed, rotor speed, and blade-pith angle. It is defined as $\partial P / \partial \theta_{c t r}$, where 

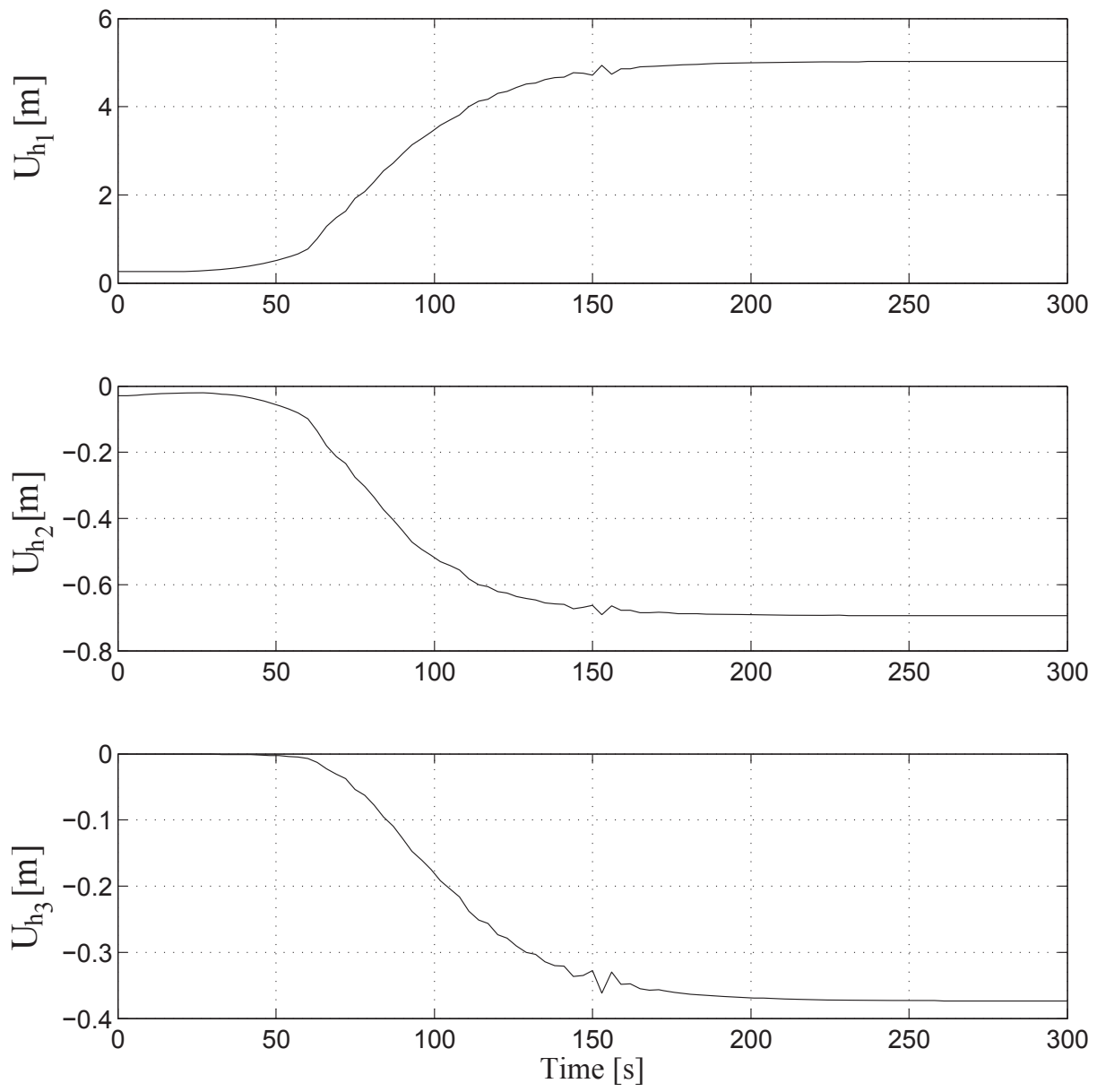

Figure 5.20. Linear displacements evolution for a point located at the $95 \%$ of the blade's referenceline during start-up.(referred to a coordinate system aligned with the rotor's plane) 



Figure 5.21. Angular displacements evolution for a point located at the $95 \%$ of the blade's reference-line during start-up.(referred to a coordinate system aligned with the rotor's plane) 

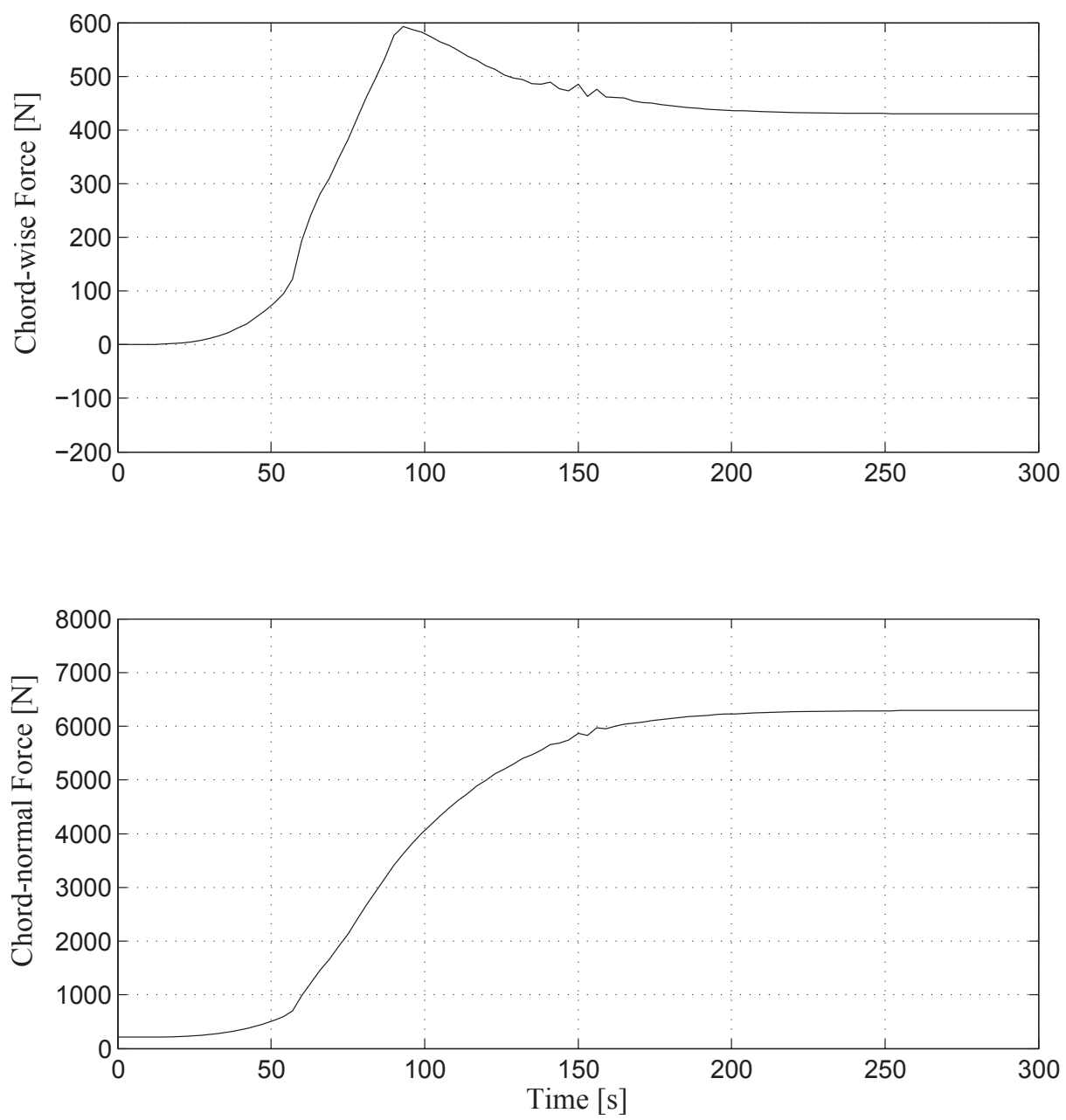

Figure 5.22. Aerodynamic forces evolution for a point located at the $95 \%$ of the blade's referenceline during start-up. 




Figure 5.23. Rotor torque evolution during start-up.

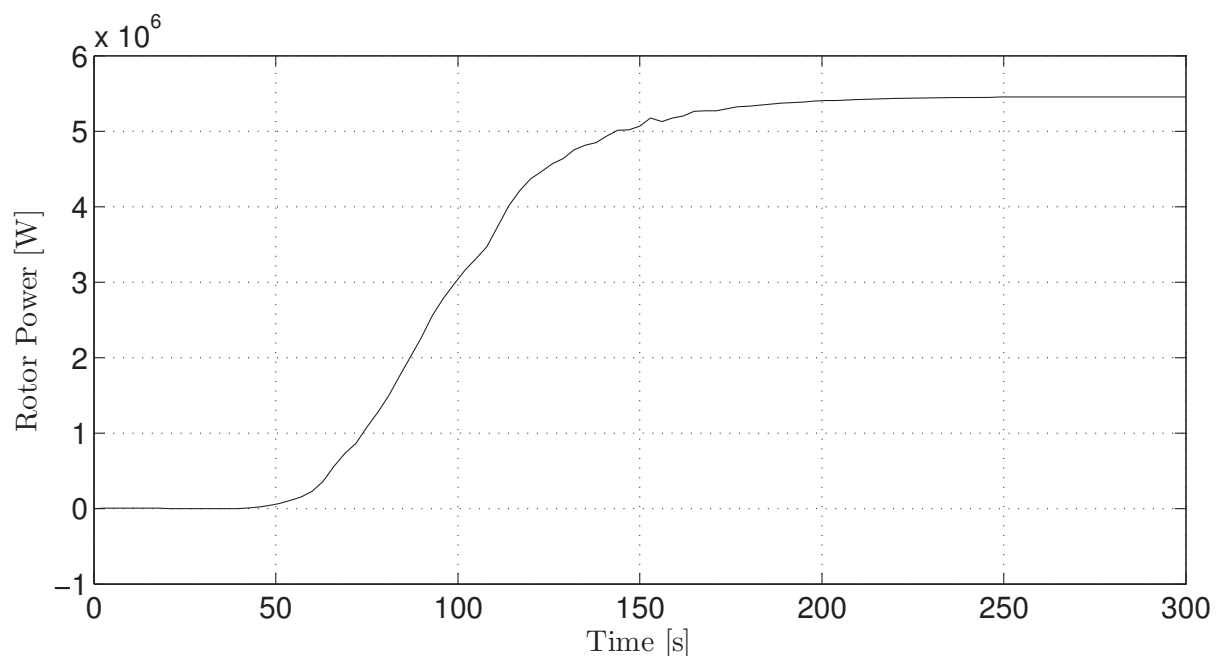

Figure 5.24. Power output evolution during start-up. 


\begin{tabular}{lll}
\hline $\begin{array}{l}\text { Wins Speed } \\
{[\mathrm{m} / \mathrm{s}]}\end{array}$ & $\begin{array}{l}\text { Pitch Angle } \\
{\left[{ }^{\circ}\right]}\end{array}$ & $\begin{array}{l}\partial P / \partial \theta_{c t r} \\
{[\text { watt/rad }]}\end{array}$ \\
\hline 11.4 (rated) $)$ & 0.000 & $-14.76 \times 10^{6}$ \\
12.0 & 2.534 & $-20.24 \times 10^{6}$ \\
13.0 & 5.271 & $-29.86 \times 10^{6}$ \\
14.0 & 7.403 & $-34.56 \times 10^{6}$ \\
15.0 & 9.214 & $-44.93 \times 10^{6}$ \\
16.0 & 10.779 & $-52.01 \times 10^{6}$ \\
17.0 & 12.224 & $-58.22 \times 10^{6}$ \\
18.0 & 13.574 & $-64.57 \times 10^{6}$ \\
19.0 & 14.842 & $-70.27 \times 10^{6}$ \\
20.0 & 16.070 & $-72.95 \times 10^{6}$ \\
21.0 & 17.265 & $-77.15 \times 10^{6}$ \\
22.0 & 18.416 & $-84.22 \times 10^{6}$ \\
23.0 & 19.517 & $-90.13 \times 10^{6}$ \\
24.0 & 20.582 & $-94.65 \times 10^{6}$ \\
25.0 & 21.622 & $-97.53 \times 10^{6}$ \\
\hline
\end{tabular}

Table 5.9

Sensitivity of Aerodynamic Power to Blade Pitch. Generated Power and rotor speed are kept constant at $5.455 \mathrm{MW}$ and $12.1 \mathrm{rpm}$ respectively.

$P$ is the output power and $\theta_{c t r}$ is the pitch control angle.

We ran a series of aeroelastic tests for steady-state operational conditions at different incoming wind speeds varying from $12 \mathrm{~m} / \mathrm{s}$ to $25 \mathrm{~m} / \mathrm{s}$, which is considered to be the cut-out wind speed. Optimizing the pitch control angle for the blades at every wind condition, we kept both the power output and rotor speed constant. Table 5.9 summarizes the results for the test, columns two and three show the optimum pitch angle and $\partial P / \partial \theta_{\text {ctr }}$ respectively.

Comparing the results in table 5.9 with the ones given in reference [16], we see that our optimum computed angles are $1^{\circ}$ to $1.5^{\circ}$ smaller. This is consistent with the fact that our model takes into consideration the coupled modes of deformation for the blade structure as well as the non linear behaviour introduced by the materials. On the aerodynamic side, the LSR-BEM model feeds back the 1-D beam model with the corresponding re-projection of the aerodynamic loads as the structure deforms. What we see here in these differences is the result of the effect of the combined deformation modes that change the alignment of the blade sections and make the aerodynamic forces, which produce the driving torque, decrease with increasing deformation as the wind speed increases. Thus, the pitch control angles required to maintain a constant output power turn out to be smaller than the ones predicted with FAST in reference [16]. 


\subsection{Blade Adaptiveness}

When the blade twists under load, the angle of attack changes, modifying the lift force, which constitutes the main component of aerodynamic load. As in an aircraft wing, this aeroelastic mechanism could be potentially dangerous if the blade structure is not properly designed. However, coupling between bending and twisting can be used to reduce extreme loads and improve fatigue performance. This is the principle of the Adaptive Blades (see [80,81], among others), where the incremental loads are reduced when, as the blade bends, the flexo-torsional modes of the blade structure produce a decrease in the blade twist, and so, the angle of attack. The level of load reduction is controlled by the level of bend-twist coupling, which depends on the blade cross-sectional geometry, the level of anisotropy in the structural material, and the material distribution [81]. Another mean to achieve bendtwist coupling is by re-adapting the geometry of the blade building it in a curved shape [82]. These two techniques can be used independently or combined, complementing each other.

Flexo-torsional or bend-twist coupling is a concept originally coming from aeronautics. Fine tuning of the relative positions between the structural torsional center of the blade section, and its aerodynamic center (conventionally located at the quarter-chord length for typical airfoils) is one of the main principles of swept wings to avoid the so-called structural divergence and self-adapt angles of attack on the wing sections. This concept was originally developed in Germany in the 1930s [83,84] and deeply researched during the days of World War II. There are two possible configurations on swept wings, the first is known as backwards sweeping. This became almost universal for light planes, jet fighters, bombers, and commercial aircraft flying under the subsonic and transonic regimes. The main characteristic of this configuration is that the aerodynamic coefficient reference point is located behind the wing section's torsional center resulting in a natural nose-down motion of the airfoil section which decreases the angle of attack with increasing aerodynamic loads. This stabilizing effect was a key factor, added to other innovations, for the success of the Messerschmitt Me 262 jet fighter of WWII which later influenced the designs of post-war aircraft in the United States such as the F-86 Sabre and the Boeing B-47 Stratojet [85-87] among others. The second possible configuration is known as forward sweeping or structural divergence. Here the aerodynamic coefficient reference point is forward to the wing torsional center causing the airfoil to increase the angle of attack in a nose-up motion as the aerodynamic loads increase. Experimental aircraft like the Grumman X-29 are based on this principle, where the aerodynamic instability of the wing configuration increased agility, but also required the use of advanced computerized control systems for piloting and composite materials on the blade structure to counteract the effects caused by the structural divergence $[88,89]$, which can potentially lead to a complete structural collapse. In addition to adaptiveness effects, swept wings help delay the drag increment caused by fluid compressibility [90,91] at high Mach number regimes. Within the scope of this work we will only focus on the structural-adaptiveness aspect of the swept-wing 


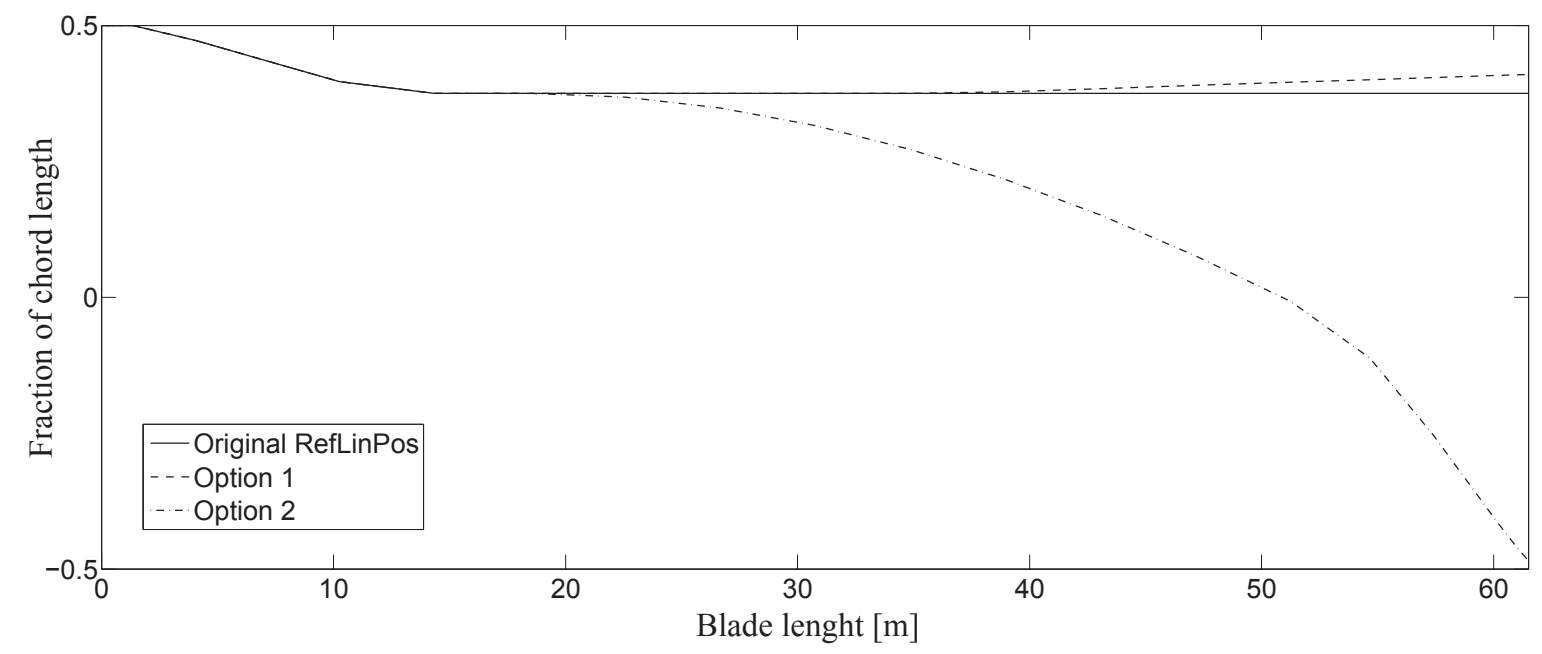

Figure 5.25. Comparison of sections pitch axis position along the blade span for three blade configurations.

concept, which is the relevant one in terms of wind-turbine blade applications.

In this section we present a series of analyses for wind turbine rotors with original blades plus two new options for blade sections arrangement along the span. The purpose of this set of experiments is to test both the blade and rotor responses under different blade geometry configurations according to the sweeping options described above. A clear advantage of seeking blade adaptiveness by sweeping is that it mostly involves geometrical re-arrangement of the blade sections along the axis of the blade mold, while materials distribution as well as plying processes keep unchanged, making it a viable option both from the performance and the economic point of view.

Based on the standard blade-sections distribution shown in table 5.4, we propose a first configuration option modifying the position of the blade's longitudinal axis (i.e. the reference line of the equivalent beam) starting at $60 \%$ of the span, from the original value of 0.375 of the chord length from the airfoil's leading edge, increasing linearly up to 0.410 at $100 \%$. This first configuration results in a slightly forward swept blade shape. The second configuration option is a more extended modification of this parameter, which now starts at $30 \%$ of the blade span, and gradually decreases from its original value towards the blade tip. For this case, the intention was to obtain the same structural behaviour as in a sweptback blade, with a minimum modification of the manufacturing process. Figure 5.25 shows a comparison of the position of the reference line between the original blade and the two new configurations, and figure 5.26 shows a superposition of the plan shape of the second blade option over the original blade.

In order to test the aeroelastic dynamics of these blade configurations under transient 


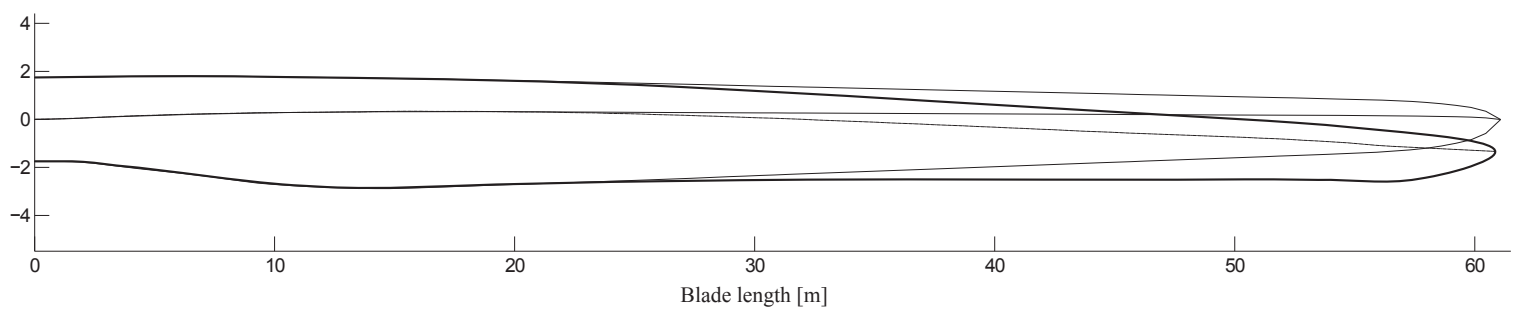

Figure 5.26. Blade configuration comparison. Original vs. swept back blade.

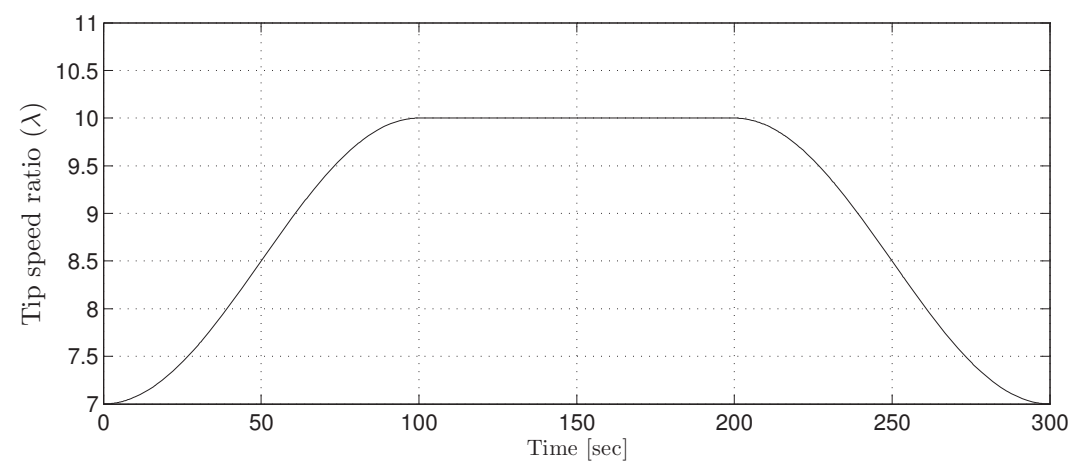

Figure 5.27. Tip speed ratio evolution during blade adaptiveness simulations. Variation of the rotational speed of the turbine at constant rated wind speed (according to table 5.6).

conditions, we designed simulation where the rotor is accelerated beyond the rated operational speed, and then returned back to its nominal regime. We start from the nominal tip speed ratio of $\lambda=7$, and let the rotor speed up to $\lambda=10$ varying only the rotational speed of the turbine. The rotor then stays in this regime for a certain period of time, where we can observe its vibrational dynamics, and afterwards it reduces its rotational speed coming back down to $\lambda=7$. This evolution could be representative of a generic transient situation in which the rotational regime of the rotor is varied as the result of the action of the control system or the mechanical response of the drive train. More specific situations involving particular control strategies would be analyzed in the future. Figure 5.27 shows the time evolution of the tip speed ratio during the 300 seconds of the simulation period. The acceleration and deceleration ramps have a sinusoidal shape.

\subsubsection{Results analysis}

Figure 5.28 shows the time evolution of the displacement of the blade in the direction perpendicular to the rotor disk $U_{h_{1}}$, at a point located at $95 \%$ of the blade span, for the three blade configurations. Figures 5.29 and 5.30 show respectively the thrust and torque exerted 
on the main shaft, and figure 5.31 shows the power output. In all these plots we could identify a common pattern of behavior for each one of the blade configurations, which is clearly consistent with the theoretical principles of the swept blades mentioned above. In the case of the original blade, the location of the blade axis at 0.375 of the chord length puts the aerodynamic center (located at 0.25 ) 0.125 of a chord length ahead of the blade axis. For the type of airfoil used in this blade, and the flow's angle of attack when the turbine operates close to nominal conditions, this puts the blade axis approximately at the center of pressure (i.e. the point of application of the aerodynamic force). If the blade axis had been located at the aerodynamic center, there would have been an aerodynamic pitch moment acting in the nose-down sense producing a twist that would have reduced the angle of attack. In other words, the original design includes a forward sweep specifically intended to minimize the torsional deformation (and hence, the change in the angle of attack) when the blade operates around the nominal regime. This also puts the blade at the limit of aeroelastic stability, which is the reason why the oscillations appearing at the moment of deceleration when the regime is stabilized at $\lambda=10$ are neither amplified nor mitigated but remain at roughly the same level. For the case of the first blade option, where the forward sweep was increased, the aeroelastic stability is further reduced to the point that, even with a slight sweep forward from the original design, the oscillations are amplified. This affects not only geometrical quantities like the flapwise deformation (see figure 5.28), but also the torque, the thrust, and the power output. If the forward sweep is further increased, we will soon reach a point where the oscillations become so large that they will induce structural failure. On the other hand, the backward swept blade, as it was expected, has the tendency of minimizing the oscillations, as could be seen in all the plots. We made a series of tests with different configurations of backward sweep, and selected the second option described above as a very interesting example on how a mitigation of those fluctuations could be achieved by this relatively simple process of re-design. Some further discussion about how those fluctuations may affect several aspects of the operational life of the turbine will be presented in the concluding section.

Finally, figure 5.32 shows the time evolution of the blade torsional deformation $\theta_{r_{1}}$, which directly affects the angle of attack (and hence, the aerodynamic loads on the blade section) initiating the chain of events that ultimately leads to the evolution of the other variables. Here we could clearly see how the oscillations in the torsion angle remain mostly constant in the original blade, but are amplified in the forward-swept blade, and quickly damped in the backward-swept blade. In a sense, the coupling of the aeroelastic combination could act either as a damper or an amplifier, depending on how the blade is designed. In the case of a damper, it would behave in the same sense as a viscous dissipation in the material. This could be related to the eigenvalues of the aeroelastic response of the blade/fluid as a system, the imaginary part of the eigenvalues would give the frequencies of the vibrational modes, while their real parts would reflect the effect either of the damping or the amplification of the aeroelastic coupling. 
This analysis also confirms the important role played by the flexo-torsional modes of deformation that our model can take into account. This requires that both the structural and the flow modules could reflect the effects of the combined modes of deformation on the aerodynamic loads exerted on the blade sections. As mentioned above, these features are absent in previous aeroelastic models like the FAST-Aerodyn suite. 



Figure 5.28. Time evolution of the displacement perpendicular to the rotor disk for a section located at $95 \%$ of the blade span, according to figure 5.27. From top to bottom, original blade, forwardswept blade, and backward-swept blade. 

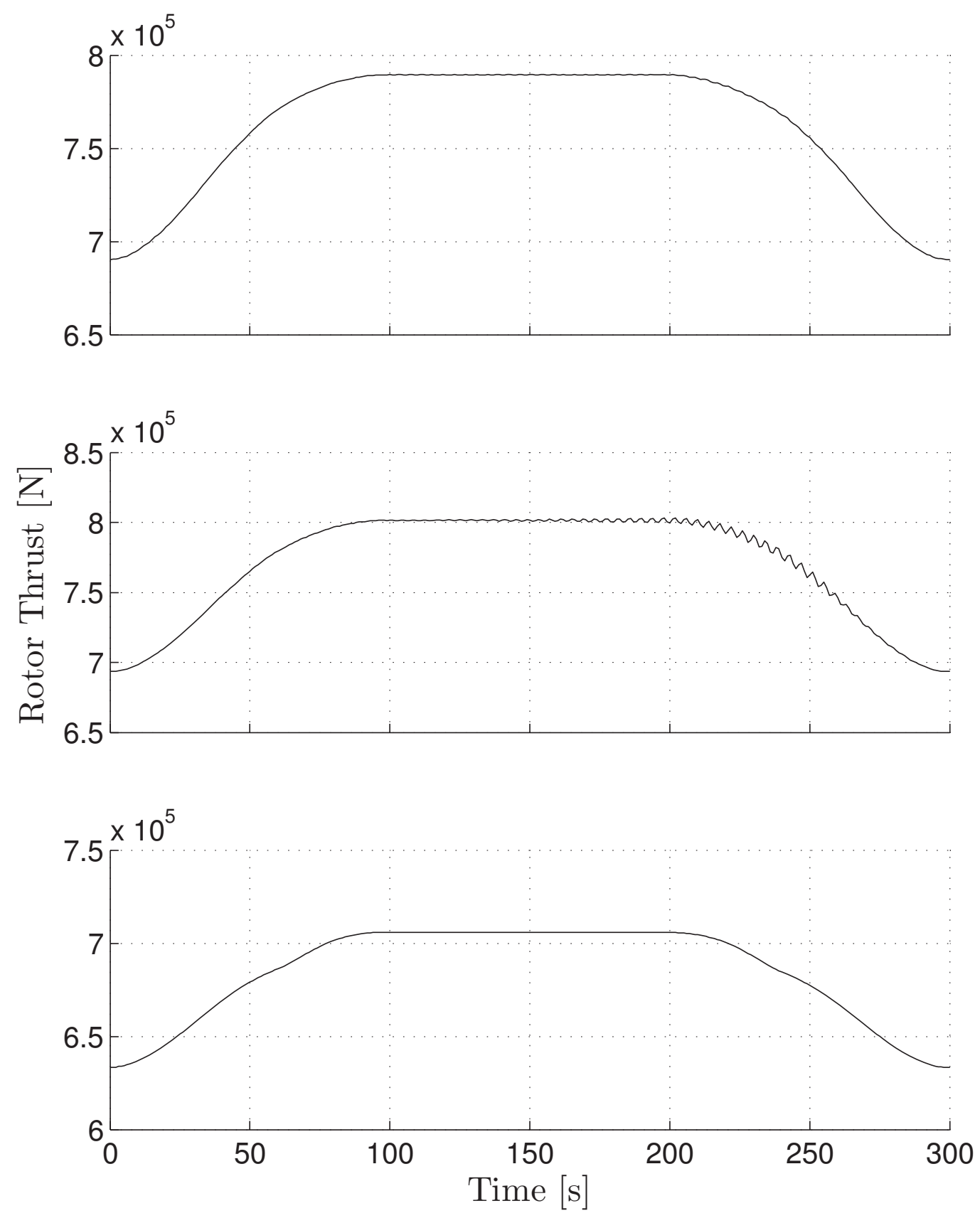

Figure 5.29. Time evolution of the rotor thrust force, according to figure 5.27. From top to bottom, original blade, forward-swept blade, and backward-swept blade. 

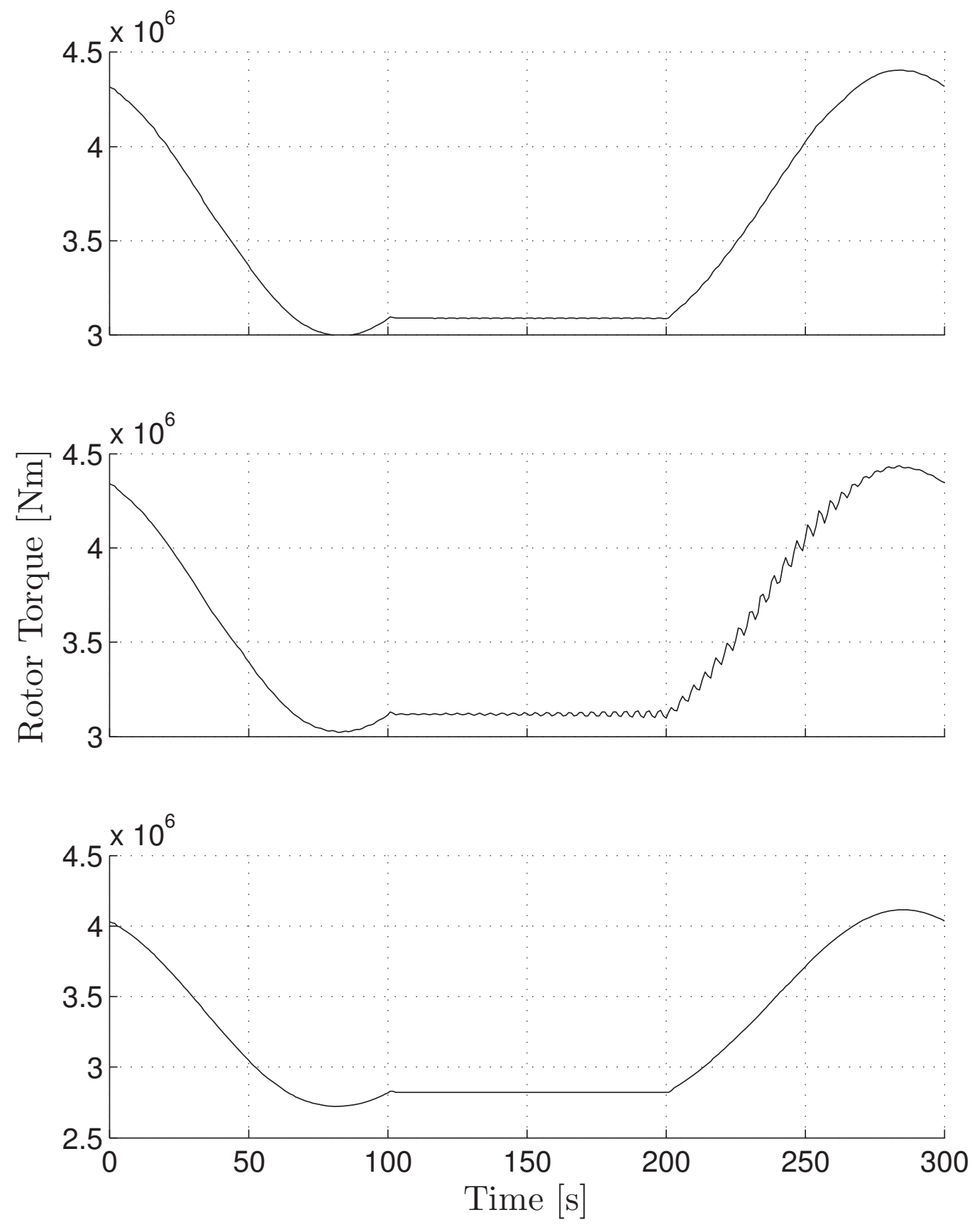

Figure 5.30. Time evolution of the rotor torque, according to figure 5.27. From top to bottom, original blade, forward-swept blade, and backward-swept blade. 

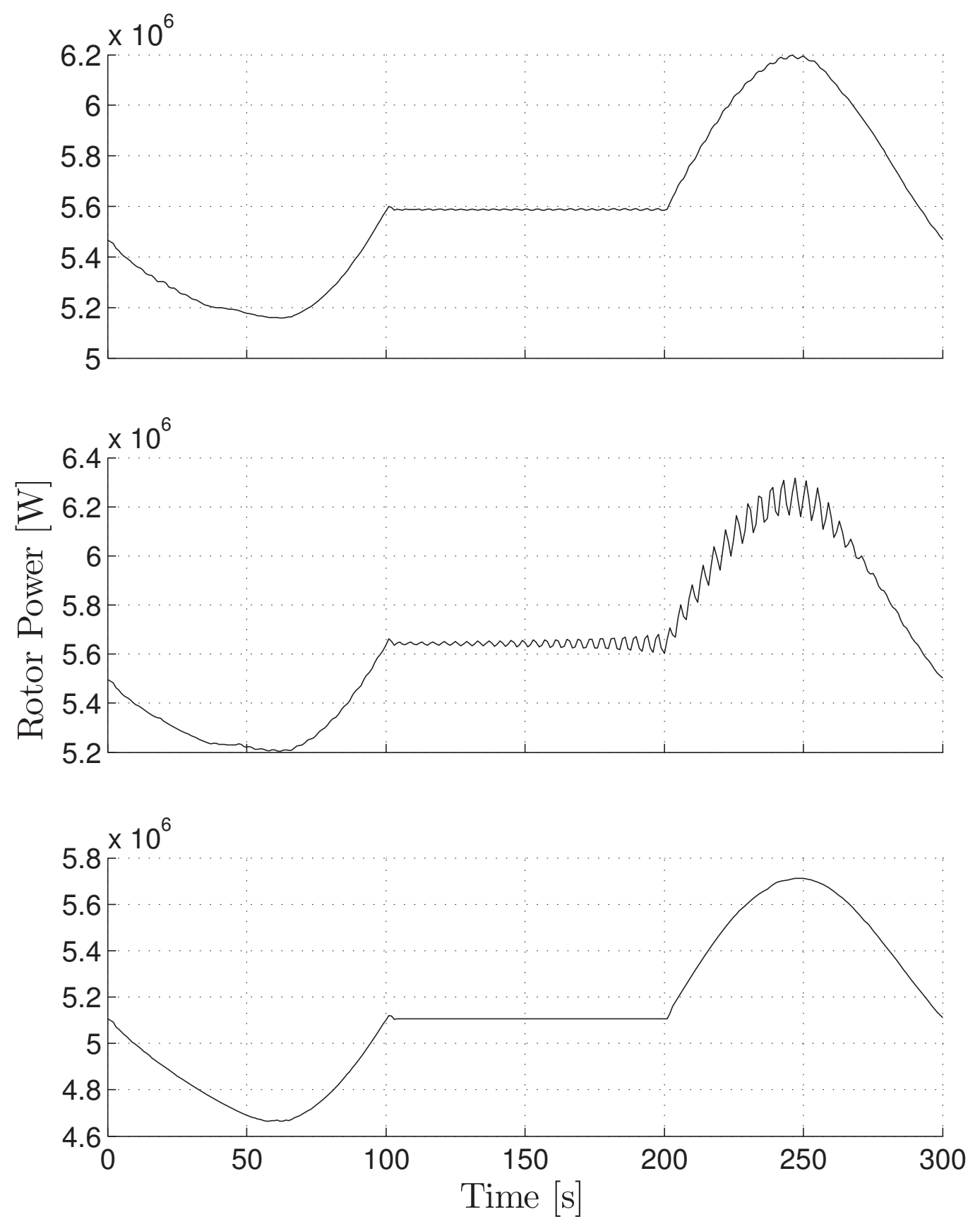

Figure 5.31. Time evolution of the rotor's output power, according to figure 5.27. From top to bottom, original blade, forward-swept blade, and backward-swept blade. 

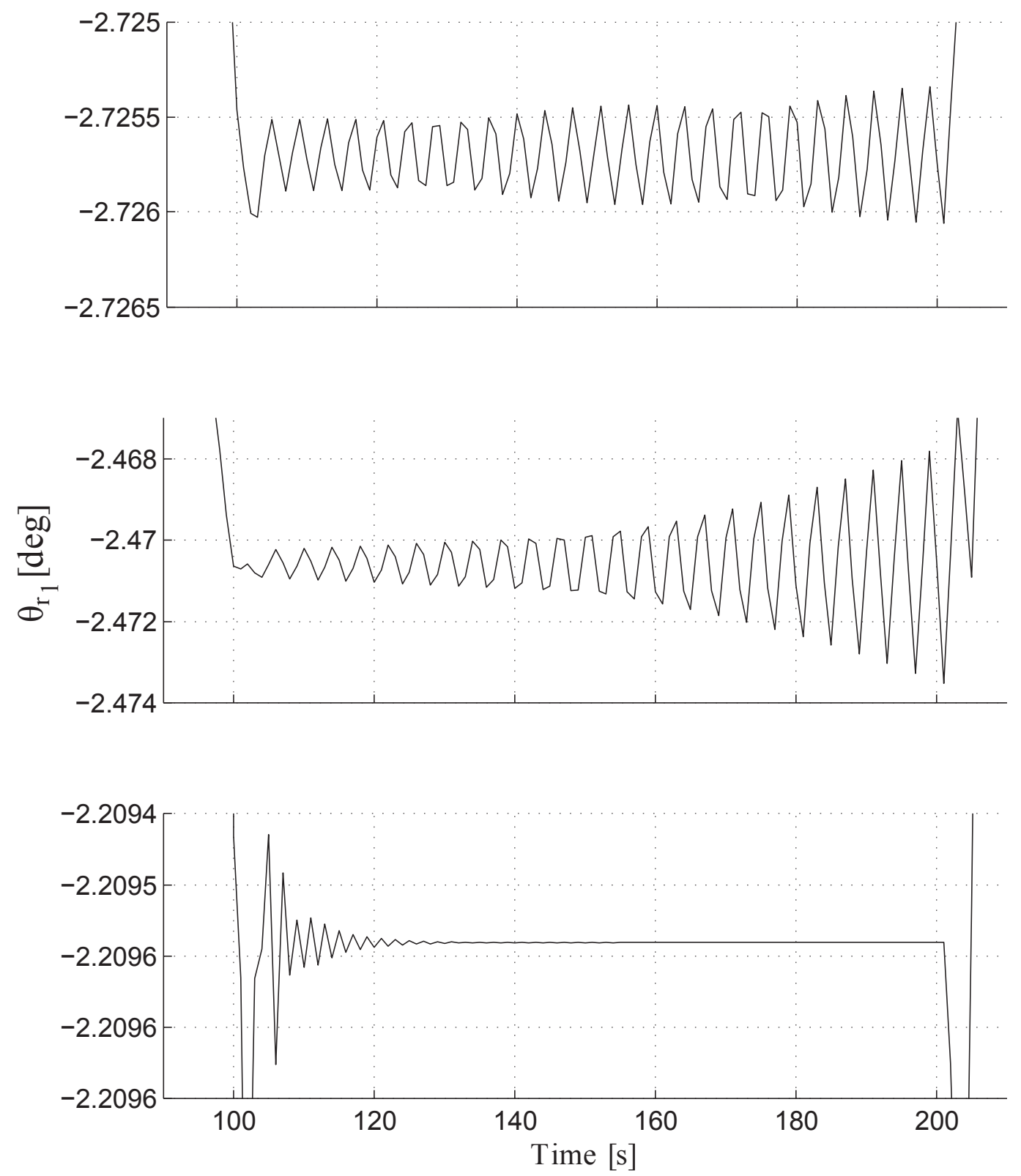

Figure 5.32. Time evolution of the blade section torsion angle for a section located at the $95 \%$ of the blade span, according to figure 5.27. From top to bottom, original blade, forward-swept blade, and backward-swept blade. 


\subsection{Gravitational forces}

As we have seen in the introduction to this work, the growing size of state-of-theart wind turbines implies technological innovations associated with the scaling. Scaling the different wind turbine components does not involve the same level of complexity and challenges. In this process, the main component to focus innovation efforts to increase productivity through enhancing energy capture is the rotor [82]. Nevertheless, if one would simply scale rotor blades without changing the fundamental design, blades would be much heavier resulting in greater structural loads [82]. To illustrate this, let's review the rotor scaling relations described in Manwell's book [26] focusing on the stresses affecting this component.

Stresses on a wind turbine rotor can be divided into three types: aerodynamic, centrifugal, and gravitational. Maximum stresses at the root of the rotor blades are bending stresses caused by aerodynamic moments. Aerodynamic forces scale with the square of the rotor radius, thus, if we multiply by the radial distance where the forces are applied, the aerodynamic moments will be proportional to the cube of the rotor radius $\left(R^{3}\right)$. In general, the blade root area moment of inertia also scales with the cube of the radius $\left(R^{3}\right)$. With this, if we divide the aerodynamic moments by the area moment of inertia, we show that rotor scaling does not affect the aerodynamic stresses as the radius dependency is the same for both players in the stress equation $\sigma_{A}=M(t / 2) / I$, where $M$ is the aerodynamic moment, $t$ is the thickness of the root and $I$ is the area moment of inertia.

Stresses due to centrifugal forces are tensile stresses, $\sigma_{C}=F_{c} / A_{c}$, where $F_{c}$ is the centrifugal force at a given blade section and $A_{c}$ is the section's area. The centrifugal force can be expressed as $F_{c}=m_{s} r_{c g} \Omega^{2}$, being $m_{s}$ the mass of the blade section, $r_{c g}$ the center of gravity distance and $\Omega$ the rotational speed of the turbine. Analyzing the dependency of all these variables with the radius when scaling the rotor, we observe that $m_{s}$ scales with $R^{3}, r_{c g}$ scales with $R$ and, as rotor scaling supposes that the tip speed ratio is kept constant, $\Omega$ scales as $R^{-1}$ making $F_{c} \sim R^{2}$. Similarly, $A_{c} \sim R^{2}$, thus, stresses due to centrifugal forces are also unchanged with rotor scaling.

On the other hand, gravitational stresses have a linear dependency with rotor scaling. Consider a blade of weight $W$ at a horizontal position. The maximum moment due to gravity will result from multiplying its weight times the center of gravity distant: $M_{g}=$ $W r_{c g}$. For sake of simplicity, let's assume a rectangular section for the blade root, thus, its moment of inertia would have the expression $I=t c^{3} / 12$. The maximum stress due to gravity $\sigma_{g}$ in the edgewise direction can be expressed as:

$$
\sigma_{g}=\frac{c W r_{c g}}{2 I}=\frac{6 W r_{c g}}{t c^{2}}
$$


Since only weight scales as $R^{3}$ while the other dimensions scale as $R$, it is demonstrated that stresses due to weight scale linearly with the radius with rotor scaling.

As the size of the state-of-the-art machine tends to increase, there will be a point from which the gravitational stresses will be comparable to the aerodynamic ones. At this point, rotor design would be facing a new constrain. Fatigue effects caused by the cyclic stresses from gravitational forces would compromise the operational life of the blades. If we want to explore new frontiers in the scaling of wind turbines it is crucial to have the tools to study and accurately predict the effects of this phenomena during the design stage. To this end, we present in this section the analysis of a fully coupled aeroelastic problem including gravitational effects on the rotor.

\subsubsection{Gravitational effects during rotor acceleration}

During our first experiments on transient conditions, and particularly under rotor accelerations, we have observed that gravitational forces can induce rumbling phenomena on the rotor blades. This rumbling doesn't necessarily indicate that a catastrophic structural failure will occur, but it is important to study their origins and effects, as further scaling of gravitational forces with size could amplify the phenomenon. During acceleration tests with the original blade, we have detected rumbling for several intermediate tip speed ratio regimes in the range from $\lambda=0$ to $\lambda=7$. The occurrence of these rumbling events appear to be very sensitive to the acceleration rate, the complexity of the blade geometry, and the properties of the materials. Here we shall analyze some results which could be assumed as representative, while more comprehensive study of this extremely rich phenomenon will be considered in the future. Figure 5.33 shows the time evolution of the aerodynamic chordnormal force on a rotor blade for an acceleration curve from $\lambda=1$ to 4 in a timespan of 350 seconds, which will serve the purpose of illustrating the rumbling effect. Here we can see that the strongest effects occur on the vicinity of $\lambda=3.7$, thus, we decided to explore deeply in this regime, doing a complete analysis of the main structural and aerodynamic variables.

Gravitational forces on a rotor blade act mostly in the chord-wise direction, with a small projection in the chord-normal sense. Thus, if we consider the action of the gravitational forces on the coordinate system of the blade section, as described on section 3.2, we shall see that they act mostly chord-wise, with a small chord-normal component. While the effects of these combined loads can be fully taken into account by our structural model, they cannot be fully represented on the current version of FAST [56]. This gives our model the unique capacity of capturing dynamic effects induced by the gravitational action.

There are several effects induced by the action of the gravitational forces on the main variables involved in the aeroelastic problem. First, there is an influence on the aerody- 


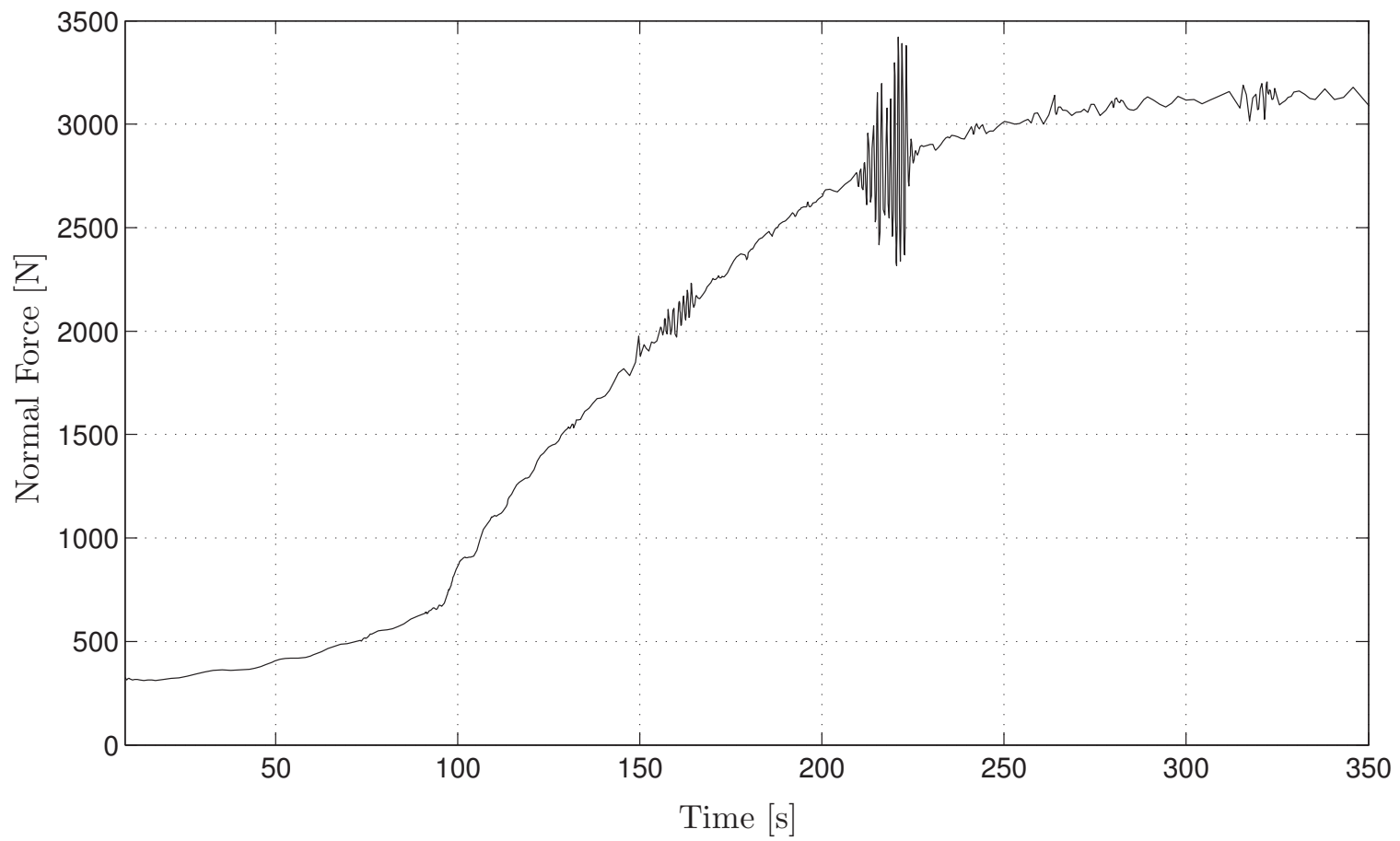

Figure 5.33. Time evolution of the aerodynamic chord-normal force on the blade under acceleration from $\lambda=1$ to $\lambda=4$ with gravitational forces included. 
namic loads which comes from the fact that the velocities of the structural deformation induced by gravity directly affect the velocity, and hence the angle of the incident flow on the blade sections $\varphi$ (see equation 3.23), which ultimately affects the angle of attack and the aerodynamic force. From the point of view of a pure structural interaction, there is also a coupled flexo-torsional mode reflected on the GTBM model which relates the flapwise displacement $U_{r_{3}}$ with the torsion $\theta_{r_{1}}$. This generates another link in aeroelastic coupling besides the changes in $\varphi$ due to the structural velocities deformations, which is not reflected on FAST. The effects on $\theta_{r_{1}}$ plus the ones on $\varphi$ directly induce changes on the blade section's angle of attack $\alpha$ which will affect the aerodynamic loads, particularly the chord-normal component $F N_{r}$. From here on we are going to see how this chain of events result in a fully aeroelastic coupled problem as the $F N_{r}$ forces feedback on the $U_{r_{3}}$ displacements, which finally closes the loop affecting the blade torsion $\theta_{r_{1}}$.

\subsubsection{Rumbling effects induced by gravitational forces}

As mentioned above, we conducted a systematic analysis on the effects of the gravitational forces on a rotor blade under a constant regime defined by a tip speed ratio equal to $3.7(\lambda=3.7)$, for a timespan of 10 minutes. In order to identify the contribution of the individual physical mechanisms involved in this coupled fluid-structure interaction problem, we will refer to figure 5.34, where the schematics of the aeroelastic transfer is described. The input of the gravitational forces effects acts as an input to the first block which represents the structural transfer. As explained before, the LSR-BEM model is able to incorporate all the information coming from the GTBM model, as an input to the block representing the aerodynamic transfer. The figure shows a switch symbol allowing to close (or not) the loop feeding the output from the aerodynamic transfer back to the structural one. Our code allows an easy way to simulate situations where the aerodynamic transfer is not fed back to the structural counterpart. We can picture this as if the blades were allowed to rotate in the vacuum, completely disaffected from the aerodynamic loads, but still affected by the gravitational field. This situation is, of course, purely virtual and it is only possible numerically, but allows us to analyze which would be the harmonic content of the structural and the aerodynamic transfer separately, discriminate the contribution of each one to the coupled aeroelastic transfer, and determine to which extent the rumbling phenomenon is related with aeroelastic coupling or with nonlinear effects of purely structural origin. We then ran the same experiments but now closing the switch indicating that the aerodynamic forces are fed back to the structural module as it would be in the real situation, allowing the complex interaction of the aeroelastic dynamics to take place. The advantage of this technique is that it puts in evidence the mechanisms that originate the vibrations, something that, when the aeroelastic problem is fully coupled, it is impossible to distinguish because all the frequencies are present together due to the feedback between the modules.

Figures 5.35 to 5.41 show the evolution of structural and aerodynamic variables as well 




Figure 5.34. Block diagram of the dynamic response of the aeroelastic system. 
as the acting gravitational and aerodynamic forces, on a blade section located at $95 \%$ of the span, for the uncoupled and the fully-coupled aeroelastic problem. Together with the time evolution of these variables, a Fourier spectral analysis is included for a timespan from 350 to 450 seconds. We specifically chose this observation window because two clear rumbling events occur here for the coupled aeroelastic situation. Superimposed to the Fourier spectrum we indicate, as a reference, the first five vibrational modes from the solution of the eigenvalue problem for the linearized ODE equations around the steady state condition at $\lambda=3.7$. The frequencies of these vibrational modes are slightly different from the ones for $\lambda=7$ (shown in table 5.7) because the deformation of the blade is different, but the dominant deformation modes still coincide. The intention of including the vibrational modes here is to provide some basis of comparison with the frequencies coming from the spectral analysis of the dynamic evolution of the fully nonlinear ODE problem. Depending on the particular conditions, some of the modes of the linearized problem could never be exited during the ODE solution, and some of them may appear close to the center of a banded peak surrounded by a much richer harmonic content, which is the result of more complex nonlinear interactions in both the structural problem and the aerodynamic one.

Figure 5.35 shows the time evolution for both the chord-wise and chord-normal components of the gravitational forces acting on the blade section. Due to the almost zero twist angle at this span position, the blade section is almost aligned with the rotor plane. This is consistent with the fact that the chord-wise component shows a simple oscillatory pattern given by the projection of the gravitational force as the blade turns with the frequency of the rotation of the main shaft at approximately $0.19 \mathrm{~Hz}$ (see the substantial peak in figure 5.35b); and it is also the reason why the normal component of the gravitational force is so small compared to the tangential component.

Analyzing the chordwise displacements we can appreciate the differences between the uncoupled and coupled situations. Three main oscillatory events are located near 170, 350 and 450 seconds as shown in figure 5.36c. As mentioned above, the observation window for our Fourier spectral analysis is located between 350 and 450 seconds, as the two events located in this region are very clear rumbling examples. The first event around 170 seconds is milder and presents certain particularities which deserve further analysis (we will return to this later on). Going back to the 350 to 450 seconds observation window, it is important to note how when the aerodynamic transfer is coupled to the structural one, locking between the two physical mechanisms appears, as can be seen in the spectra on figures $5.36 \mathrm{~d}$, $5.37 \mathrm{~d}$, and $5.38 \mathrm{~d}$, and more markedly in figures $5.39 \mathrm{~d}, 5.40 \mathrm{~d}$ and $5.41 \mathrm{~d}$. Here the frequencies for the dynamic problem show a certain correlation with several of the vibrational modes from the linearized solution, in general the first and second, with a mild correlation of the third that could be noticed in figure 5.41d. This correlation clearly links the rumbling events with the coupling of the aeroelastic problem, as the two events completely disappear when the aeroelastic coupling is switched off (see figures ). In addition, we can reproduce by the following figures the connection line between the deformation modes as we previ- 
ously described in the introduction to this section. Effects on the chordwise displacements are transferred to the flapwise direction (see figure 5.37) through the flexural-flexural deformation modes, which will directly influence the aerodynamic loads through changes in the angle of the flow incident on the blade section, $\varphi$ (see figure 5.39). Flexo-torsional modes affect the $\theta_{1}$ angle (see figure 5.38) which will influence directly the angle of attack, $\alpha$,(figure 5.40) and will be reflected finally on the aerodynamic chord-normal forces as can be seen in figure 5.41. Thus, this locking between the two physical mechanisms, the flow and the structure, comes through two different paths related to a correct representation of the combined modes of deformation, both of them requiring the already mentioned unique characteristics of our model to be taken into account.

There are many other oscillatory events along the total timespan related with the nonlinear transferences of the system which are not so clearly associated with a structural frequency, nevertheless these oscillations also present a correlation among the deformations. An example of this is the event located around 170 seconds of the timespan. As for the previously described rumbling events, this one is also present only when the aeroelastic problem is coupled, which can be seeing in the spectral plots for both situations in the figures. Observing the different variables, we could speculate that this event is triggered by an energy accumulation originated in the flapwise direction, $U_{3}$, in figure 5.37, showing a small oscillation near 170 seconds. That energy could have been transmitted to $\theta_{1}$ by a flexo-torsional mode, as figure 5.38 shows the same effect, and later re-transmitted to $U_{2}$, which suddenly reflects that energy close to 200 seconds, as shown in figure 5.36. In order to fully determine the nature of this transfer, a more comprehensive analysis will be required (we are going to return to this in our outlook for further work in chap 6). But whichever the details of the physical interaction, this event shows the importance and usefulness of a dynamic analysis as it could evidence events in the oscillatory behavior which are impossible to capture by just analysing a linearized solution for an aeroelastic steady state and its vibrational modes. 


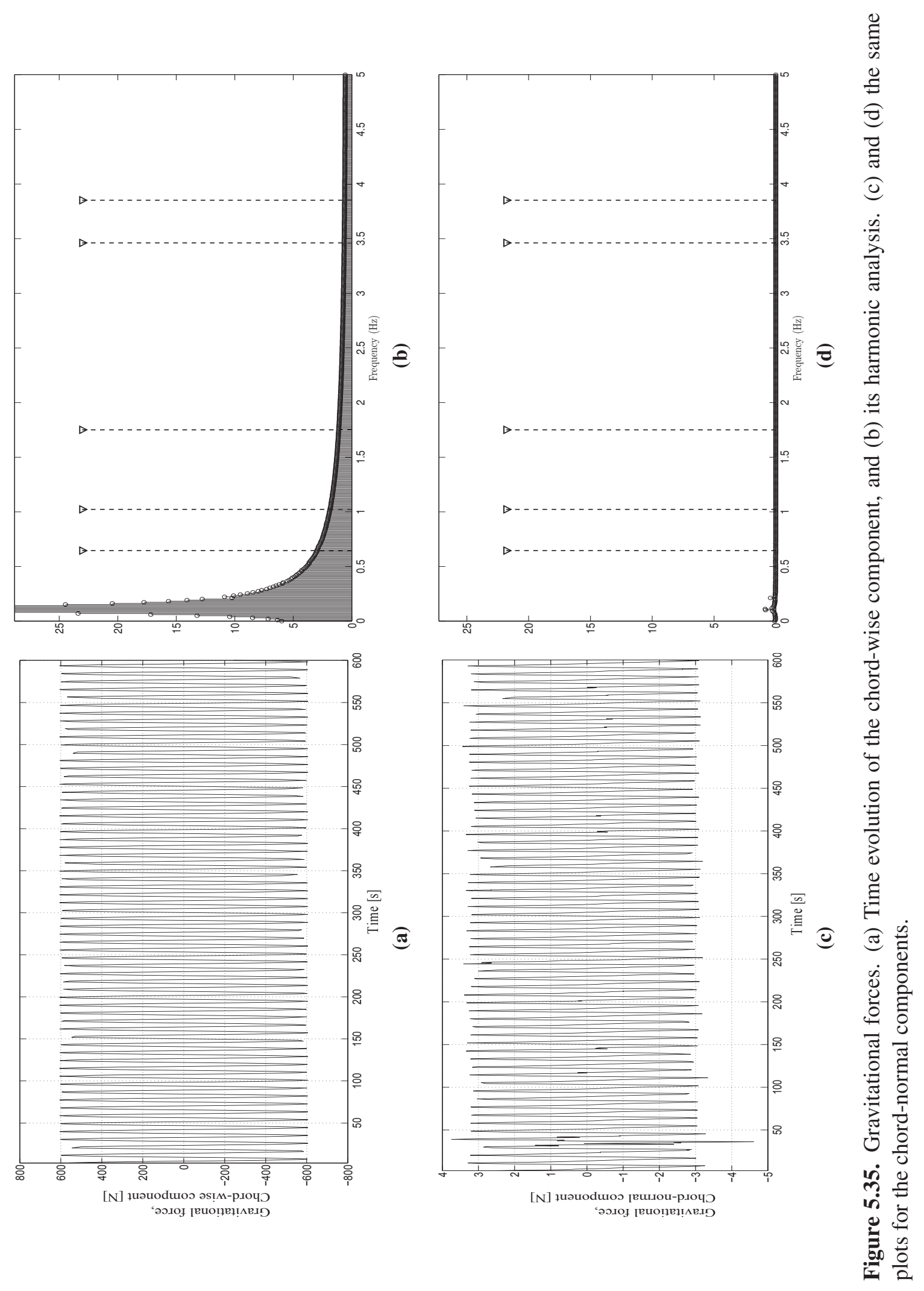




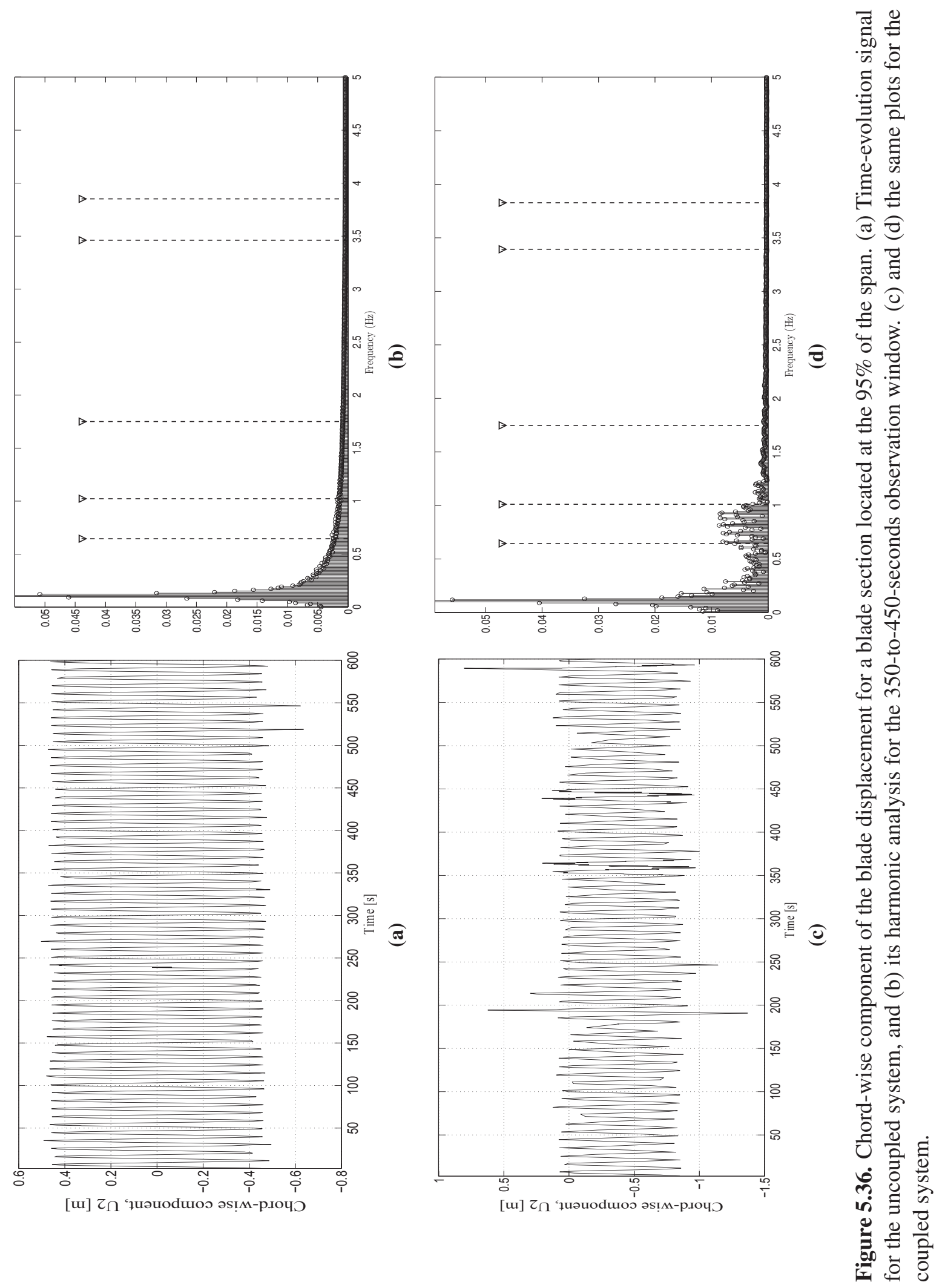






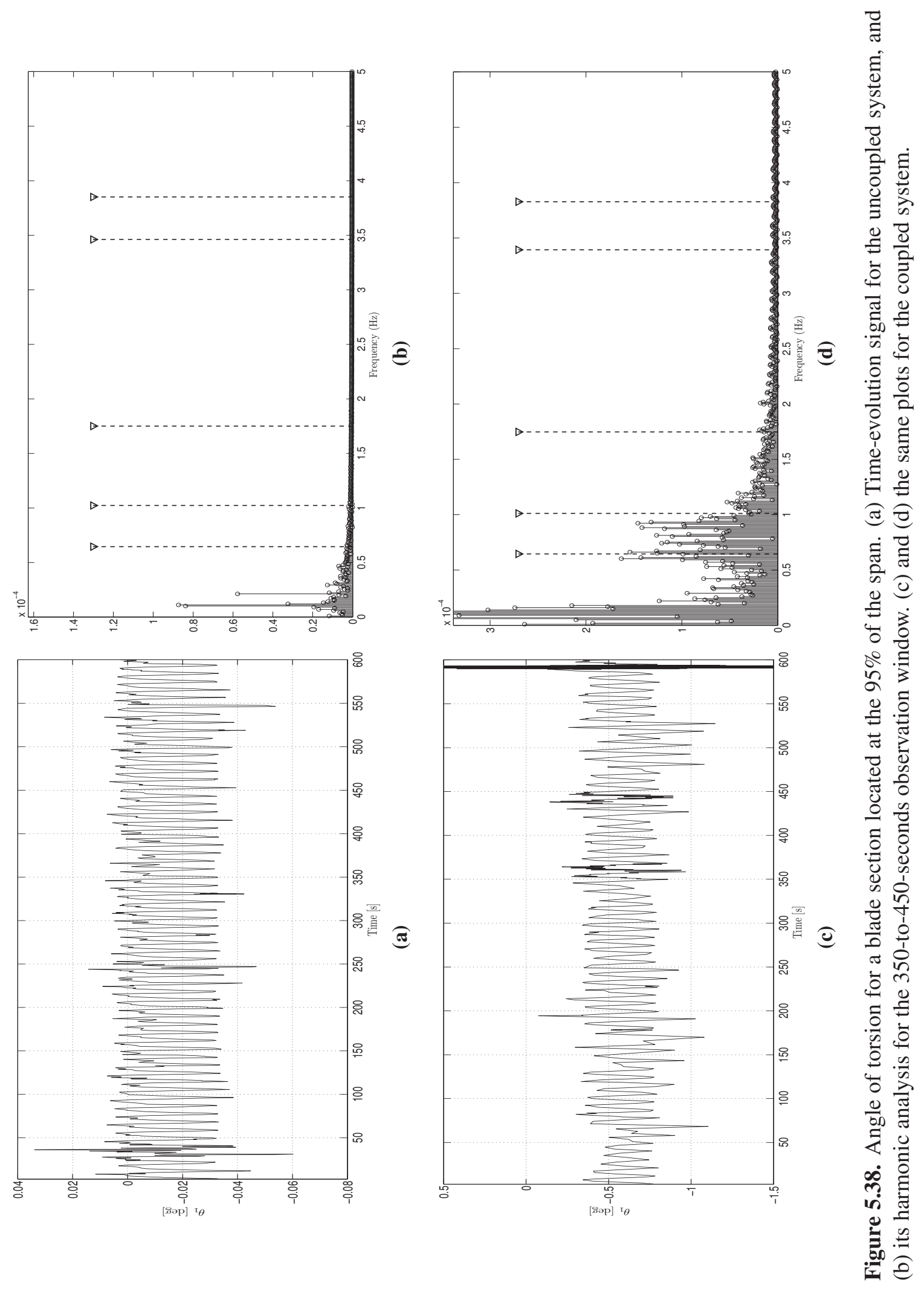




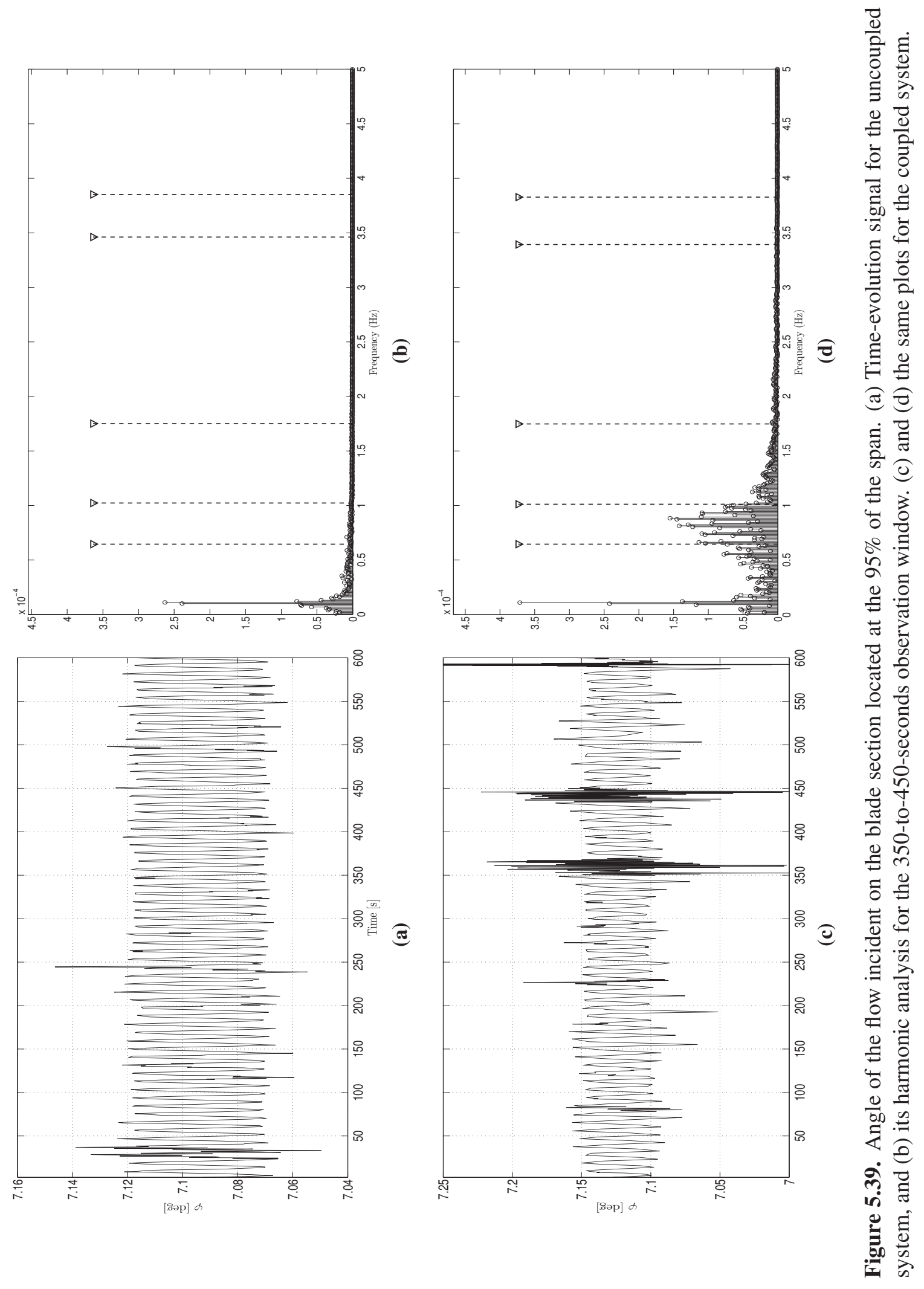









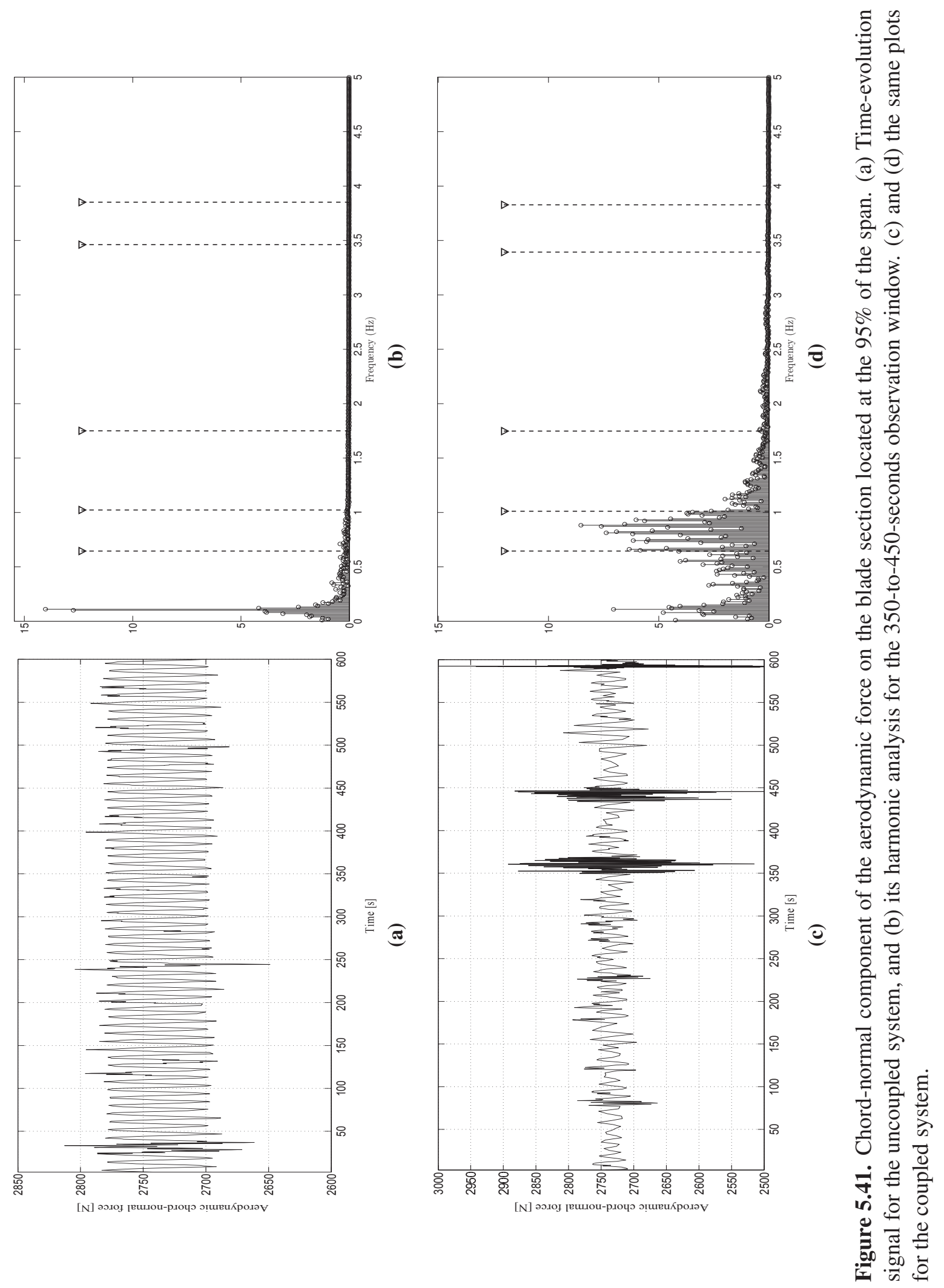




\section{Conclusions}

In this dissertation we have successfully developed a very powerful computational tool for the aeroelastic analysis of wind-turbine blades. Due to the particular features mentioned above in terms of a full representation of the combined modes of deformation of the blade as a complex structural part and their effects on the aerodynamic loads, it constitutes a substantial advancement ahead the state-of-the-art aeroelastic models currently available, like the FAST-Aerodyn suite. Here, we also include the results of several experiments on the NREL-5MW blade, which is widely accepted today as a benchmark blade, together with some modifications intended to explore the capacities of the new code in terms of capturing features on blade-dynamic behavior, which are normally overlooked by the existing aeroelastic models. In this regards, some further remarks could be added to the observations already made in the corresponding sections dedicated to those experiments in Chapter 5:

A more detailed look at the deformation modes shows some interesting characteristics. For example, the top plot of figures 5.15 shows displacements for the first deformation mode. Analysing this plot we observe that the dominant displacements are $\mathbf{U}_{\mathbf{1}}$ and $\boldsymbol{\theta}_{2}$ (see also table 5.7). Nevertheless, as the blade sections are non-symmetric, materials are non isotropic and the blade's axis has an initial torsion, the deformation modes are not always pure. Instead, we see also a small displacement on $\mathrm{U}_{2}$ and $\boldsymbol{\theta}_{1}$, meaning that a combination with flexion in-plane of the rotor disk exists. This combinations can be seen for all blade modes. Analysing the second mode we can see a dominant flexural deformation in the inplane direction, while modes 3, 5 and 10 show a dominant out-plane flexural mode. On the other hand mode 4, in figure 5.16, shows a combined flexo-torsional mode as $\mathrm{U}_{2}$ and $\boldsymbol{\theta}_{3}$ are the dominant displacements. This bend-twist coupling can be seen also for modes 6 and 8 in table 5.7. This analysis puts in evidence one of the key advantages of this structural model capturing the couplings between strain measures that give rise to combined deformation modes under fully coupled fluid-structure interaction problems employing the LSR-BEM model for the aerodynamic loads.

Another interesting point concerning the reliability of the code could be obtained from a comparison of the values of the displacements of the blade section at $95 \%$ of the span

obtained from the aeroelastic analysis for the steady state conditions at nominal regime 
(figure 5.11), with the same magnitudes at the end of the acceleration process for the starup test reported in figure 5.20, section 5.2.4. The difference between the two cases is in the order of $10^{-4}$ for the mostly flapwise displacement in the direction normal to the rotor's plane, and $10^{-5}$ in the other two directions. This issue is not trivial, as we are considering two results obtained by completely different approaches: The first comes from an iterative aeroelastic solution using a linearized version of the equations for the structural problem, which ultimately converges to the steady state condition after a set of intermediate iterative steps which have no physical meaning but are, essentially, numerical steps intended to tackle the nonlinear problem. The second, on the other hand, comes from a time integration process of the full set of nonlinear equations for the whole aeroelastic problem starting with the rotor at rest. Taking into account the extreme complexity and sensitivity of the models involved, the very low deference reported is remarkable.

Regarding to the analysis of blade adaptiveness in section 5.3, even tough fluctuations in the aerodynamic loads may not necessarily lead to catastrophic failure (unless a very high level of structural divergence is inherent to the design), their mitigation is critical to avoid structural fatigue, which is a determining factor in blade lifespan. There are also many other components on the wind turbine that are directly affected by the level of fluctuation in the aerodynamic loads on the blades. For instance, the thrust and torque on the main shaft (see figures 5.29 and 5.30) directly affect the design of several wind-turbine components like the gearbox, couplings, bearings and the rotor's main shaft [26,27]. Moreover, the fluctuations in the torque are also reflected as fluctuations in power output (see figure 5.31), this may affect several other subsystems of the turbine like the generator, the power electronics, and other electrical systems involved, with effects which, if strong enough, may even propagate to the electric grid.

As an outlook for further work, in view of the results presented here, we may identify several lines of research which will be worthwhile to explore:

In terms of the adaptive-blade concept, the possibility of modifying the orientation of the fibers in the composite laminates used in blade construction, together with other modifications in the layout of the internal structure of the blade, could now be expanded and complemented by suitable modifications of the aerodynamic design in such a way of increasing the effects of vibrational mitigation reported here. It could also be possible to achieve some degree of power limitation at high wind speeds, which in combination with flaps, micro-tabs, or other small flow-control devices, would reduce the size (or completely eliminate) the need of expensive pitch-control actuators. To this end, further experiments with swept-back blades with a fully curvilinear axis seem to be particularly promising.

In terms of the analysis of the rumbling phenomena and other vibrational effects triggered by the presence of gravitational forces, the initial results presented here suggest a very rich field of research on which we just opened the door. A more comprehensive set 
of experiments with different blade configurations, working under a wide range of operational conditions (which would constitute a project in itself), would reveal important information about fatigue effects to guide future designs, especially for of extra-large blades of lightweight construction. 


\section{REFERENCES}

(1) F. P. L. Chen, L. Lago, Perspectives on innovative concepts in wind-power generation, Energy for Sustainable Development (2011) 398-410.

(2) N. Hodge, A snapshot of the global wind industry, [cited February 2010]. Available at http://www.greenchipstocks.com/articles/ wind-energy-companies/273 (2010).

(3) WWEA, World Wind Energy Report 2009, Report, World Wind Energy Association, 2010. Www . wwindea. org.

(4) Global Wind Energy Council, Global wind statistics 2011 [internet], [cited March 2012]. Available at http://www.gwec.net/fileadmin/images/ News/Press/GWEC_-_Global_Wind_Statistics_2011.pdf (2012).

(5) NREL, Wind Power Today, Report DOE/GO-102005-2115, U.S. Department of Energy, 2005.

(6) Enercon E-126 website [internet], [cited February 2012]. Available at http:// www. enercon. de/en-en/66.htm (2012).

(7) E. de Vries, Thinking bigger: Are there limits to turbine size?, Renewable Energy World 8 (2005) 42-55.

(8) A. D. Hansen, F. Iov, F. Blaabjerg, L. H. Hansen, Review of contemporary wind turbine concepts and their market penetration, Wind Engineering 28 (2004) 247-263.

(9) L. H. Hansen, L. Helle, F. Blaabjerg, E. Ritchie, S. Munk-Nielsen, H. Bindner, P. Sørensen, B. Bak-Jensen, Conceptual survey of generators and power electronics for wind turbines, RISØ National laboratory, 2001.

(10) P. Moriarty, A. Hansen, AeroDyn theory manual, National Renewable Energy Laboratory (2005). 
(11) D. Laino, A. Hansen, User's Guide to the Wind Turbine Aerodynamics Computer Software AeroDyn, Report for National Renewable Energy Laboratory under subcontract No. TCX-9-29209-01 (2002).

(12) A. Otero, Análisis no-lineal del comportamiento estructural de sistemas avanzados de conversión eoloeléctrica, Ph.D. thesis, Facultad de Ingeniería, Universidad de Buenos Aires, National Lybrary, Buenos Aires, Argentina, 2008. In spanish.

(13) A. D. Otero, F. L. Ponta, Structural analysis of wind-turbine blades by a generalized timoshenko beam model, Journal of Solar Energy Engineering 132 (2010) 011015.

(14) D. W. Hodges, Nonlinear Composite Beam Theory, AIAA, Reston, Virginia, 2006.

(15) W. Yu, D. H. Hodges, Generalized Timoshenko theory of the variational asymptotic beam sectional analysis, J. American Helicopter Society 50 (2005) 46-55.

(16) J. Jonkman, S. Butterfield, W. Musial, G. Scott, Definition of a 5-MW reference wind turbine for offshore system development, National Renewable Energy Laboratory, NREL/TP-500-38060 (2009).

(17) L. F. Shampine, Numerical solution of ordinary differential equations, Chapman \& Hall, New York, USA, 1994.

(18) L. F. Shampine, M. K. Gordon, Computer Solution of Ordinary Differential Equations: the Initial Value Problem, W. H. Freeman, San Francisco, 1975.

(19) I. G. L.F. Shampine, S. Thompson, Solving ODEs with MATLAB, Cambridge University Press, 2003.

(20) F. L. Ponta, The KLE method: a velocity-vorticity formulation for the Navier-Stokes equations, J. Applied Mechanics 73 (2006) 1031-1038.

(21) G. F. C. A. M. Valli, A. L. Coutinho, Control strategies for timestep selection in finite element simulation of incompressible flows and coupled reaction-convectiondiffusion processes, International Journal for numerical methods in fluids 47 (2005) 201-231.

(22) L. Shampine, Implementation of implicit formulas for the solution of odes, SIAM Journal on Scientific and Statistical Computing 1 (1980) 103.

(23) L. Shampine, M. Reichelt, The matlab ode suite, SIAM journal on scientific computing 18 (1997) 1-22.

(24) J. Jonkman, Minutes from the aerodyn overhaul kick-off meeting [internet], [cited June 2009]. Available at http://wind.nrel.gov/public/ jjonkman/AeroDynoverhaulKickOffMeeting (2008). 
(25) H. Glauert, Airplane propellers, Aerodynamic theory 4 (1935) 169-360.

(26) J. Manwell, J. McCowan, A. Rogers, Wind Energy Explained: theory, design and application, Chichester ; New York : Wiley, 2002.

(27) T. Burton, Wind Energy Handbook, Wiley, 2001.

(28) H. Glauert, A general theory of the autogyro., British ARC 1111 (1926).

(29) D. Pitt, D. Peters, Theoretical prediction of dynamic-inflow derivatives, Vertica 5 (1981) 21-34.

(30) J. Leishman, Principles of helicopter aerodynamics, Cambridge University Press, Cambridge, UK, 2006.

(31) C. Bak, H. Aagaard Madsen, J. Johansen, Influence from blade-tower interaction on fatigue loads and dynamics (poster), in: Wind energy for the new millennium. Proceedings. 2001 European wind energy conference and exhibition (EWEC'01), pp. 2-6.

(32) S. Powles, The effects of tower shadow on the dynamics of a horizontal-axis witn turbine, Wind Engineering 7 (1983) 26-42.

(33) K. Rao, Classical mechanics, Universities Press, 2003.

(34) H. Goldstein, Classical Mechanics, 2nd ed., Addison-Wesley, 1980.

(35) L. S. B. Siciliano, L. Villani, Robotics: modelling, planning and control, Springer Verlag, 2009.

(36) IEC, Wind Turbine Generator Systems - Part 13: Measurement of mechanical loads, Report IEC/TS 61400-13, International Electrotechnical Commission (IEC), 2001.

(37) Frenet-serret formulas [internet], [cited December 2011]. Available at http: / / en . wikipedia.org/wiki/Frenet-Serret_formulas (2011).

(38) F. Yamaguchi, Curves and surfaces in computer aided geometric design, SpringerVerlag Berlin, 1988.

(39) R. L. Burden, J. D. Faires, Numerical analysis, Brooks Cole, 1998.

(40) J. H. Mathews, K. D. Fink, Numerical methods using Matlab, Prentice Hall, 1999.

(41) B. Lock, Townend, An extension of the vortex theory of airscrews with applications to airscrews of small pitch, including experimental results, Aeronautical Research Committee, R\&M 1014 (1925) 1 to 49. 
(42) R. Wilson, Aerodynamic behavior of wind turbines, Wind Turbine Technology, Fundamental Concepts of Wind Turbine Engineering, D. Spera, ed (1994).

(43) M. Buhl, A new empirical relationship between thrust coefficient and induction factor for the turbulent windmill state, National Renewable Energy Laboratory, 2005.

(44) C. Hansen, Nwtc design codes (airfoilprep) [internet], [cited December 2011]. Available at http://wind.nrel.gov/designcodes/preprocessors/ airfoilprep/Last modified 09-March-2010 (2011).

(45) Z. Du, M. Selig, A 3-D stall-delay model for horizontal axis wind turbine performance prediction, in: AIAA, Aerospace Sciences Meeting and Exhibit, 36 th, and 1998 ASME Wind Energy Symposium, Reno, NV; UNITED STATES, Reston, VA/New York: American Institute of Aeronautics and Astronautics, Inc./ASME International, pp. 9-19.

(46) A. Eggers, Modeling of yawing and furling behavior of small wind turbines, in: 2000 ASME Wind Energy Symposium, 19 th, AIAA, Aerospace Sciences Meeting and Exhibit, 38 th, Reno, NV, pp. 1-11.

(47) J. Leishman, T. Beddoes, A generalised model for airfoil unsteady aerodynamic behaviour and dynamic stall using the indicial method, in: 42nd. Annual Forum of the American Helicopter Society, Washington D. C.

(48) L. Viterna, D. Janetzke, Theoretical and experimental power from large horizontalaxis wind turbines, NASA STI/Recon Technical Report N 82 (1982).

(49) J. Leishman, S. Aerodynamicist, T. Beddoes, A. Specialist, A Semi-Empirical Model for Dynamic Stall, Journal of the American Helicopter Society 34 (1989) 3.

(50) J. Leishman, T. Beddoes, A generalized model for unsteady aerodynamic behaviour and dynamic stall using the indicial method, J. Am. Helicopter Soc 36 (1990) 14-24.

(51) K. Pierce, Wind turbine load prediction using the beddoes-leishman model for unsteady aerodynamics and dynamic stall, Master's thesis, The University of Utah, 1996.

(52) J. Minnema, Pitching moment predictions on wind turbine blades using the BeddoesLeishman model for unsteady aerodynamics and dynamic stall, Master's thesis, The University of Utah, 1998.

(53) H. A. RL. Bisplinghoff, R. Halfman, Aeroelasticity, Addison-Wesley, Cambridge, MA, 1955.

(54) B. Thwaites, Incompressible Aerodynamics, Oxford University Press, 1960. 
(55) M. Hansen, Aerodynamics of wind turbines, Earthscan/James \& James, 2008.

(56) J. Jonkman, M. Buhl Jr, Fast users guide, Rep. No. NREL/EL-500-38230, NREL, Golden, Colorado, USA (2005).

(57) D. A. Griffin, Blade System Design Studies Volume I: Composite Technologies for Large Wind Turbine Blades, Report SAND2002-1879, Sandia National Laboratories, 2002.

(58) V. L. Berdichevsky, Variational-asymptotic method of constructing a theory of shells, J. App. Math. and Mech. 43 (1979) 664-687.

(59) W. Yu, D. H. Hodges, V. Volovoi, C. E. S. Cesnik, On Timoshenko-like modeling of initially curved and twisted composite beams, Int. J. Sol. and Struct. 39 (2002) 5101-5121.

(60) K. J. Bathe, Finite element procedures, Prentice Hall, Englewood Cliffs, New Jersey, USA, 1996.

(61) D. H. Hodges, Geometrically exact, intrinsic theory for dynamics of curved and twisted anisotropic beams, AIAA Journal 41 (2003) 1131-1137.

(62) M. J. Patil, M. Althoff, Energy-consistent, Galerkin approach for the nonlinear dynamics of beams using mixed, intrinsic equations, in: AIAA/ASME/ASCE/AHE/ASC Structures, Structural Dynamics and Material Conference, Reston, Virginia, USA, AIAA, 2006, pp. 1-9.

(63) G. E. Karniadakis, E. T. Bullister, A. T. Patera, A spectral element method for solution of two- and three-dimensional time-dependent incompressible navier-stokes equations, in: Finite Element Methods for Nonlinear Problems, Springer-Verlag, New York/Berlin, 1985, p. 803.

(64) A. T. Patera, A spectral element method for fluid dynamics: laminar flow in a channel expansion, J. Comput. Phys. 54 (1984) 468-488.

(65) L. E. Malvern, Introduction to the mechanics of a continuous medium, Prentice Hall, 1969.

(66) L. P. Kollár, G. S. Springer, Mechanics of Composites Structures, Cambridge University Press, New York, USA, 2003.

(67) V. V. Vasiliev, E. V. Morozov, Mechanics and Analysis of Composite Materials, Elsevier, Oxford, UK, 2001.

(68) G. Wempner, D. Talaslidis, Mechanics of solids and shells: theories and approximations, CRC Press LLC, Boca Raton, USA, 2003. 
(69) J. Reddy, Mechanics of Laminated Composite Plates. Theory and Analysis, CRC Press, Boca Raton, USA, 1997.

(70) H. Kooijman, C. Lindenburg, D. Winkelaar, E. van der Hooft, DOWEC 6 MW predesign, Technical Report, ECN-CX-01-135, Energy Research Center of the Netherlands, Petten, 2003.

(71) C. Lindenburg, Aeroelastic modelling of the lmh64-5 blade, ECN, Petten, December (2002).

(72) Iea wind, subtask 2: Research for deeper waters [internet], [cited December 2011]. Available at http://www. ieawind.org/AnnexXXIII/Subtask2. html (2011).

(73) Upwind website [internet], [cited December 2011]. Available at http://www . upwind.eu/default.aspx (2011).

(74) J. Jonkman, S. Butterfield, P. Passon, T. Larsen, T. Camp, J. Nichols, J. Azcona, A. Martinez, Offshore code comparison collaboration within iea wind annex xxiii: Phase ii results regarding monopile foundation modeling, in: 2007 European Offshore Wind Conference \& Exhibition, 4-6 December 2007, Berlin, Germany.

(75) P. Passon, M. Kühn, S. Butterfield, J. Jonkman, T. Camp, T. Larsen, OC3-Benchmark exercise of aero-elastic offshore wind turbine codes, in: Journal of Physics: Conference Series, volume 75, IOP Publishing, p. 012071.

(76) W. A. Timmer, R. P. J. O. M. van Rooij, Summary of the Delft University wind turbine dedicated airfoils, in: 41st Aerospace Sciences Meeting and Exhibit, AIAA, Reno, Nevada.

(77) W. A. Timmer, Personal communication, 2007.

(78) I. TPI Composites, Parametric study for large wind turbine blades, Report SAND2002-2519, Sandia National Laboratories, 2002.

(79) D. J. L. Jason Jonkman, Nwtc design codes adams2ad [internet], [cited December 2011]. Available at http://wind.nrel.gov/designcodes/ simulators/adams2ad/ (2011).

(80) D. A. Griffin, Evaluation of Design Concepts for Adaptive Wind Turbine Blades, Report SAND2002-2424, Sandia National Laboratories, 2002.

(81) J. Locke, I. Contreras Hidalgo, The Implementation of Braided Composite Materials in the Design of a Bend-Twist Coupled Blade, Report SAND2002-2425, Sandia National Laboratories, 2002. 
(82) NREL, 20\% Wind Energy by 2030: Increasing Wind Energy's Contribution to U.S. Electricity Supply, Report DOE/GO-102008-2567, U.S. Department of Energy, 2008.

(83) H.-U. Meirer, German development of the swept wing 1935-1945. (originally published in german as die deutsche luftahrt die pfeilflügelentwicklung in deutschland bis 1945, bernard \& graefe verlag, 2006), AIAA Library of Flight (2010).

(84) J. D. Anderson, A History of Aerodynamics, McGraw Hill, 1997.

(85) D. O'Connell, Messerschmitt Me 262: The Production Log 1941-1945., Classic Publications, Leicestershire, UK, 2006. ISBN 1-903223-59-8.

(86) J. Foreman, S. Harvey, The Messerschmitt Me 262 Combat Diary., Air Research Publications, Surrey, UK, 1990. ISBN 1-871187-30-3.

(87) M. Blair, Evolution of the f-86, in: AIAA Evolution of Aircraft Wing Design Symposium.

(88) R. Thruelsen, The Grumman Story, Praeger Publishers, Inc., New York, 1976. ISBN 0-275-54260-2.

(89) J. Winchester, Grumman X-29. X-Planes and Prototypes, Amber Books Ltd, London, 2005. ISBN 1-904687-40-7.

(90) H. C. J.G. Barmby, I. Garrick, Study of effects of sweep on the flutter of cantilever wings, National Advisory Committee for Aeronautics, 1951.

(91) L. B. W.F. Hilton, G. Berk, High-speed aerodynamics, Longmans, Green and Co., 1952. 


\section{APPENDICES}




\section{APPENDIX A. RECOVERY OF THREE DIMENSIONAL VARIABLES}

Once the $1 \mathrm{D}$ problem is solved as seen in section 4.2 , the inverse process to the dimensional reduction technique takes place. In this process we recover the variables which represent the actual behavior of the 3D solid. Thus, warping, three-dimensional displacements, strain, and stresses are computed for every blade section from the 1D model solution, i.e. the measures of deformation, the displacements of the reference line, and the rotations of the blade sections.

\section{A.1 Computation of the sections' warping}

According to ([14] chapter 4, section 2) and [12], the warping array at the nodes of the mesh are expressed as:

$$
\mathbf{V}=\left(\overline{\mathbf{V}}_{0}+\overline{\mathbf{V}}_{1 R}\right) \varepsilon+\overline{\mathbf{V}}_{1 S} \boldsymbol{\varepsilon}^{\prime}
$$

where $\varepsilon$ are the equivalent measures of deformation from the Bernoulli theory. Thus, we need to express now these equivalent measures of deformation in terms of the Timoshenko theory as explained in [14] and [12].

To obtain the warping we need the measures of deformation $\varepsilon$ and their first derivatives along the blade's reference line. These $\varepsilon$ are strain, torsion, and curvatures and their derivatives $\boldsymbol{\epsilon}^{\prime}$. Also, the measures of deformation corresponding to shear $\gamma_{s}$ with the first and second derivatives are needed. Deformations are computed from the obtained forces and moments coming from the 1D model applying the constitutive relations. Derivatives of the deformations are computed by a linear operator at every node of the $1 \mathrm{D}$ finite element mesh. For more details on this technique, please refer to [12].

As the dimensional reduction model uses the deformation energy asymptotically up to 
order $\mathcal{O}\left(\frac{h^{2}}{l^{2}}\right)$, equation A.1 allows us to recover warping up to first order in $\frac{h}{l}$. Yu and Hodges [15] use the forces and moments obtained at the beam sections to compute the measures of deformation and their derivatives applying the constitutive relations. To this end, they calculate the derivatives of forces and moments through the equilibrium equations in the beam. This methodology turns out to be complicated as one must know up to the third derivative of the distributed and inertial forces and moments [12]. The method we use in this work, presented by Dr. Otero in [12], has the advantage of being able to compute the measures of deformation directly from the 1D model as well as their derivatives using its discretization.

\section{A.2 Computation of stresses and deformations}

In this section we will describe the computations of the deformation tensor at the points of the blade sections. As we previously mentioned, we will need the section's warping and their derivatives with respect of the axial coordinate of the blade. Warping computations were covered under section A.1. To obtain their derivatives, we first compute the derivatives of variables $\epsilon$ and $\gamma_{s}$ as in [12] and then calculate:

$$
\mathbf{V}^{\prime}=\left(\overline{\mathbf{V}}_{0}+\overline{\mathbf{V}}_{1 R}\right) \varepsilon^{\prime}+\overline{\mathbf{V}}_{1 S} \varepsilon^{\prime \prime}
$$

Then, with $\varepsilon$, warping and their derivatives, we compute the components of the Jaumann-Biot-Cauchy deformation tensor at the nodes as:

$$
\overline{\boldsymbol{\Gamma}}=\overline{\boldsymbol{\Gamma}}_{h} \mathbf{V}+\overline{\boldsymbol{\Gamma}}_{\varepsilon} \varepsilon+\overline{\boldsymbol{\Gamma}}_{R} \mathbf{V}+\overline{\boldsymbol{\Gamma}}_{l} \mathbf{V}^{\prime}
$$

being

$$
\begin{gathered}
\overline{\boldsymbol{\Gamma}}=\left[\begin{array}{lllllll}
\Gamma_{11}^{1} & 2 \Gamma_{12}^{1} & 2 \Gamma_{13}^{1} & \Gamma_{22}^{1} & 2 \Gamma_{23}^{1} & \Gamma_{33}^{1} & \cdots \\
\cdots & \Gamma_{11}^{n} & 2 \Gamma_{12}^{n} & 2 \Gamma_{13}^{n} & \Gamma_{22}^{n} & 2 \Gamma_{23}^{n} & \Gamma_{33}^{n}
\end{array}\right]^{T}
\end{gathered}
$$

an array with the components of the deformation tensor at the nodes of the finite element mesh of the section, noted by the superscript, and $\overline{\boldsymbol{\Gamma}}_{h}, \overline{\boldsymbol{\Gamma}}_{\varepsilon}, \overline{\boldsymbol{\Gamma}}_{R}$ and $\overline{\boldsymbol{\Gamma}}_{l}$ matrices which, given the warping, their derivatives, and the measures of deformation, are used to compute $\Gamma_{h} \mathbf{w}$, $\Gamma_{\varepsilon} \varepsilon, \Gamma_{R} \mathbf{w}$ and $\Gamma_{l} \mathbf{w}^{\prime}$ respectively. This matrices are obtained according to derivation and re-projection techniques explained in [12], chapter 2. 
To optimize computational resources, equation A.3 can be rewritten as:

$$
\overline{\boldsymbol{\Gamma}}=\underbrace{\left[\left(\overline{\boldsymbol{\Gamma}}_{h}+\overline{\boldsymbol{\Gamma}}_{R}\right)\left(\overline{\mathbf{V}}_{0}+\overline{\mathbf{V}}_{1 R}\right)+\overline{\boldsymbol{\Gamma}}_{\varepsilon}\right]}_{\hat{\boldsymbol{\Gamma}}_{1}} \varepsilon+\underbrace{\left[\left(\overline{\boldsymbol{\Gamma}}_{h}+\overline{\boldsymbol{\Gamma}}_{R}\right) \overline{\mathbf{V}}_{1 S}+\overline{\boldsymbol{\Gamma}}_{l}\left(\overline{\mathbf{V}}_{0}+\overline{\mathbf{V}}_{1 R}\right)\right]}_{\hat{\boldsymbol{\Gamma}}_{2}} \varepsilon^{\prime}+\underbrace{\overline{\boldsymbol{\Gamma}}_{l} \overline{\mathbf{V}}_{1 S}}_{\hat{\boldsymbol{\Gamma}}_{3}} \varepsilon^{\prime \prime} .
$$

In this way, matrices $\hat{\Gamma}_{1}, \hat{\Gamma}_{2}$ and $\hat{\Gamma}_{3}$ will be characteristic of each section, thus they can be computed a priori.

Once the components of the deformation tensor are computed, we obtain the stress tensor applying the constitutive relations at each node of the finite element mesh [12,14]. In this operation, the deformation tensor has to be rotated in order to obtain stresses consistently expressed in the material's coordinate system. For the particular case of two elements with different materials sharing common nodes, the stress computation has to be repeated using the constitutive relations of each element. Thus, we will obtain two different stresses for the same nodes. This is the typical example of a common boundary between two layers of a laminate where certain nodes show different stiffnesses for both materials. The difference in stresses is called inter-lamina stress and could originate failure of the whole laminate, thus inter-lamina stress is critical when applying a failure theory model.

Due to the theoretical limitation in the order of the deformation energy, recovered stresses and deformations will correspond to the deformation energy of order 2 in $\frac{h}{l}$. To

obtain stresses and deformations of a higher order, the analysis would need an extreme re-elaboration of the involved theory, nevertheless the current order seems to be enough as indicated in [14].

\section{A.3 Displacements of the blade sections}

In this section we will show how the displacements of the blade section's points are obtained using the displacements of the blade reference line and the rotations of the blade sections, both covered in section 4.2.

According to [14] and [12], the displacements of the blade section's points can be expressed as:

$$
\underline{\mathbf{U}}\left(X^{1}, X^{2}, X^{3}\right)=\underline{\mathbf{u}}+X^{\alpha}\left(\underline{\mathbf{C}}^{t B}-\underline{\mathbf{I}}\right) \cdot \underline{\mathbf{B}}_{\alpha}+w_{i} \underline{\mathbf{C}}^{t B} \cdot \underline{\mathbf{B}}_{i},
$$

where $\underline{\mathbf{u}}$ is a vector containing the displacements of the blade's reference line. If we express 
the previous equation in the undeformed coordinate system, $R$, described in chapter 4 , we have:

$$
U_{j}\left(X^{1}, X^{2}, X^{3}\right)=u_{j}+X^{\alpha}\left(C_{\alpha j}^{t B}-\delta_{\alpha j}\right)+w_{i} C_{i j}^{t B},
$$

where $\mathbf{C}^{t B}$ is the linear operator which rotates from $\underline{\mathbf{B}}_{i}$ to $\underline{\mathbf{t}}_{i}$. Matrix $\mathbf{C}^{t B}$ can be obtained by subsequent rotations from the $\underline{\mathbf{B}}_{i}$ coordinate system to $\underline{\mathbf{b}}_{i}$, associated with the Timoshenko theory, and later to the Bernoulli coordinate system $\underline{\mathbf{t}}_{i}$ as:

$$
\mathbf{C}^{t B}=\mathbf{C}^{t b} \mathbf{C}^{b B}
$$

where matrix $\mathbf{C}^{t b}$ depends on the deformation measures $2 \gamma_{12}$ and $2 \gamma_{13}$, both obtained from the 1D model [14], [12].

\section{A.4 Other applications of the dimensional reduction model}

Shear and strain center of a section

The shear center of a section is defined as the point where the application of a force perpendicular to the beam axis, produces no torsion in the section [14,59]. The position can be calculated from the compliance matrix (the inverse of the stiffness matrix) of the section given the constitutive relation $\bar{\gamma}=\overline{\mathbb{C}} \overline{\mathbf{F}}$. In matrix form:

$$
\left[\begin{array}{c}
\gamma_{11} \\
2 \gamma_{12} \\
2 \gamma_{13} \\
\kappa_{1} \\
\kappa_{2} \\
\kappa_{3}
\end{array}\right]=\left[\begin{array}{llllll}
C_{11} & C_{12} & C_{13} & C_{14} & C_{15} & C_{16} \\
C_{12} & C_{22} & C_{23} & C_{24} & C_{25} & C_{26} \\
C_{13} & C_{23} & C_{33} & C_{34} & C_{35} & C_{36} \\
C_{14} & C_{24} & C_{34} & C_{44} & C_{45} & C_{46} \\
C_{15} & C_{25} & C_{35} & C_{45} & C_{55} & C_{56} \\
C_{16} & C_{26} & C_{36} & C_{46} & C_{56} & C_{66}
\end{array}\right]\left[\begin{array}{c}
F_{1} \\
F_{2} \\
F_{3} \\
M_{1} \\
M_{2} \\
M_{3}
\end{array}\right]
$$

To obtain $\zeta_{2}$ and $\zeta_{3}$, the coordinates of the shear center on a section located at $X^{1}$ on a beam with lenght $L$, we need to consider two traverse forces $\hat{F}_{2}$ and $\hat{F}_{3}$ applied at the end of the beam. Thus, the expression for the moments will be: 


$$
\begin{aligned}
& M_{1}=\hat{F}_{3} \zeta_{2}-\hat{F}_{2} \zeta_{3}, \\
& M_{2}=-\hat{F}_{3}\left(L-X^{1}\right), \\
& M_{3}=\hat{F}_{2}\left(L-X^{1}\right) .
\end{aligned}
$$

We need to find the values of $\zeta_{2}$ and $\zeta_{3}$ so that the torsion is zero, meaning that the fourth line in equation A.8 is null. Rearranging in terms of forces $\hat{F}_{2}$ and $\hat{F}_{3}$ we can express this condition as:

$$
\left[C_{24}-C_{44} \zeta_{3}+C_{46}\left(L-X^{1}\right)\right] \hat{F}_{2}+\left[C_{34}+C_{44} \zeta_{2}-C_{45}\left(L-X^{1}\right)\right] \hat{F}_{2}=0 .
$$

As forces $\hat{F}_{2}$ and $\hat{F}_{3}$ are arbitrary, the position of the shear center can be obtained as:

$$
\begin{aligned}
\zeta_{2} & =-\frac{C_{34}}{C_{44}}+\frac{C_{45}}{C_{44}}\left(L-X^{1}\right), \\
\zeta_{3} & =\frac{C_{24}}{C_{44}}-\frac{C_{46}}{C_{44}}\left(L-X^{1}\right) .
\end{aligned}
$$

These expressions show that the shear center is not a main property of the section for beams with flexo-torsional coupled modes, i.e. $C_{45} \neq 0$ and $C_{46} \neq 0$, and that the position varies along the beam. On the other hand, for beams without flexo-torsional coupled modes the position of the shear center is given by:

$$
\begin{aligned}
\zeta_{2} & =-\frac{C_{34}}{C_{44}}, \\
\zeta_{3} & =\frac{C_{24}}{C_{44}},
\end{aligned}
$$

being in this case a main property of the section. Despite this fact and for beams with flexo-torsional coupled modes, the generalized shear center is usually defined as a main property of the section through equation A.11.

Analogously, the strain center is defined as the point where no flexion is produced when an axial force is applied, meaning where stretches are decoupled from curvatures $[12,14]$. 
Given an axial force $\hat{F}_{1}$ applied at a point with coordinates $\bar{\zeta}_{2}$ and $\bar{\zeta}_{3}$ the flexural moments are:

$$
\begin{aligned}
& M_{2}=-\hat{F}_{1} \bar{\zeta}_{2}, \\
& M_{3}=\hat{F}_{1} \bar{\zeta}_{3} .
\end{aligned}
$$

Thus, equating the fifth and sixth line in equation A.8 and regrouping terms, we arrive at:

$$
\begin{aligned}
& {\left[C_{15}+C_{55} \bar{\zeta}_{3}-C_{56} \bar{\zeta}_{2}\right] \hat{F}_{1}=0} \\
& {\left[C_{16}+C_{56} \bar{\zeta}_{3}-C_{66} \bar{\zeta}_{2}\right] \hat{F}_{1}=0}
\end{aligned}
$$

Finally, the strain center coordinates $\bar{\zeta}_{2}$ and $\bar{\zeta}_{3}$ are obtained by equating to zero the terms between brackets and solving the system of equations.

These properties are useful from a technical standpoint at the design stages and can be used to optimize the loading states. For example, in rotating beams where centrifugal forces are relevant, if the strain center coincides with the section's center of mass, the centrifugal force won't cause flexural moment and thus the only resulting efforts would be tensile. Another example would be the airfoil sections of a wind turbine blade, here the intention would be to make the shear center and the aerodynamic center of the section coincident, thus, the aerodynamic forces won't cause torsional moments that would result in variations of the section's angle of attack. In the event that the aerodynamic center would be ahead of the shear center, the aerodynamic forces would produce the effect of augmenting the torsional moment, increasing the angle of attack and as a consequence increasing the aerodynamic forces even more. This phenomena known as structural divergence is covered under chapter 5, section 5.3. On the other hand, the coupling modes effects could be beneficial in some cases allowing self-adaptiveness to certain structural configurations (see section 5.3).

\section{Shear stiffness correction factors}

One of the hypothesis of the Timoshnko's beam theory is that a beam section remains

planar after deformation. This hypothesis induces errors in the computation of the shear stiffness. The theory assumes that the components of the deformation tensor corresponding 
to shear distortions out of the section are constant $[12,14]$. This violates the angular momentum conservation as, at the contour of the section, the deformation tensor wouldn't be symmetric. To overcome this problem one should use correction factors for the shear stiffness coming from the Timoshenko theory. These correction factors depend on the shape of the section and can differ for each direction.

In the dimensional reduction technique (see chapter 4) we obtained the stiffness matrix without any restrictive hypothesis for the deformations on the section. Therefore, shear stiffnesses will be exact according to the precision of the method. Thus, we could use this results to compute the correction factors for the shear stiffnesses. Although it would be more precise to use the complete stiffness matrix rather than using it to obtain the factors to only correct the shear stiffnesses, it could come in handy for some specific applications. Also, it could be a good way to validate the model against other 3D theories more complex than Timoshenko's.

The correction factors for the shear stiffness can be defined as:

$$
c_{\beta}=\frac{S_{\beta \beta}}{G\langle 1\rangle}
$$

where $c_{\beta}$ is the shear correction factor in the direction of coordinate $X^{\beta}, S_{\beta \beta}$ is the shear stiffness obtained from the dimensional reduction technique, $G$ is the shear modulus and $\langle 1\rangle$ is the section's area. Thus, $G\langle 1\rangle$ is the stiffness obtained from the classic Timosheko Theory $[12,14]$. 


\section{APPENDIX B. COPYRIGHT AGREEMENTS}




\section{B.1 Copyright statement for Chapter 1}

Pictures from chapter 1 are reproduced from Wikimedia Commons, a freely licensed media file repository. Files are licensed under the Creative Commons Attribution-Share Alike 2.0 and/or 3.0 Unported license. According to these license, permission is granted to:

1. Share, copy, distribute and transmit the work.

2. Remix, adapt the work.

Under the following conditions:

1. Attribution You must attribute the work in the manner specified by the author or licensor (but not in any way that suggests that they endorse you or your use of the work).

2. share alike If you alter, transform, or build upon this work, you may distribute the resulting work only under the same or similar license to this one.

\section{ATTRIBUTIONS:}

- Picture from figure 1.1a was taken by Hans Hillewaert. Source and permissions availables at: http://en.wikipedia.org/wiki/File:Windmill_DI_ ( Thornton_Bank).jpg, under CC-BY-SA-3.0.

- Picture from figure $1.1 \mathrm{~b}$ was taken by the Jumanji Solar's photostream. Source and permissions availables at: http://tr.wikipedia.org/wiki/Dosya: Enercon-e126.jpg, under CC-BY-2.0.

- Picture from figure 1.2 was taken by Korona B. Source and permissions availables at: http://en.wikipedia.org/wiki/File:Farma_wiatrak。C3 $\circ B 3 w \_B u k o w s k o \_. J P G$, under CC-BY-SA-3.0.

- Picture from figure 1.4 was taken by Paul Anderson. Source and permissions availables at: http://en.wikipedia.org/wiki/File:Turbine_ Blade_Convoy_Passing_through_Edenfield.jpg, under CC-BY-2.0. 


\section{B.2 Copyright statement for Chapter 3}

Figures 3.2 and 3.4 are reproductions of figures 3.6 and 3.13 respectively from "T. Burton, D. Sharpe, N. Jenkins and E. Bossanyi. Wind Energy Handbook, 2nd Edition, 2011". The contract agreement with the publisher is included here, stating that permission was granted to reuse the figures 3.6 and 3.13.

\section{JOHN WILEY AND SONS LICENSE \\ TERMS AND CONDITIONS}

Mar 05, 2012

This is a License Agreement between Lucas Lago ("You") and John Wiley and Sons ("John Wiley and Sons") provided by Copyright Clearance Center ("CCC"). The license consists of your order details, the terms and conditions provided by John Wiley and Sons, and the payment terms and conditions.

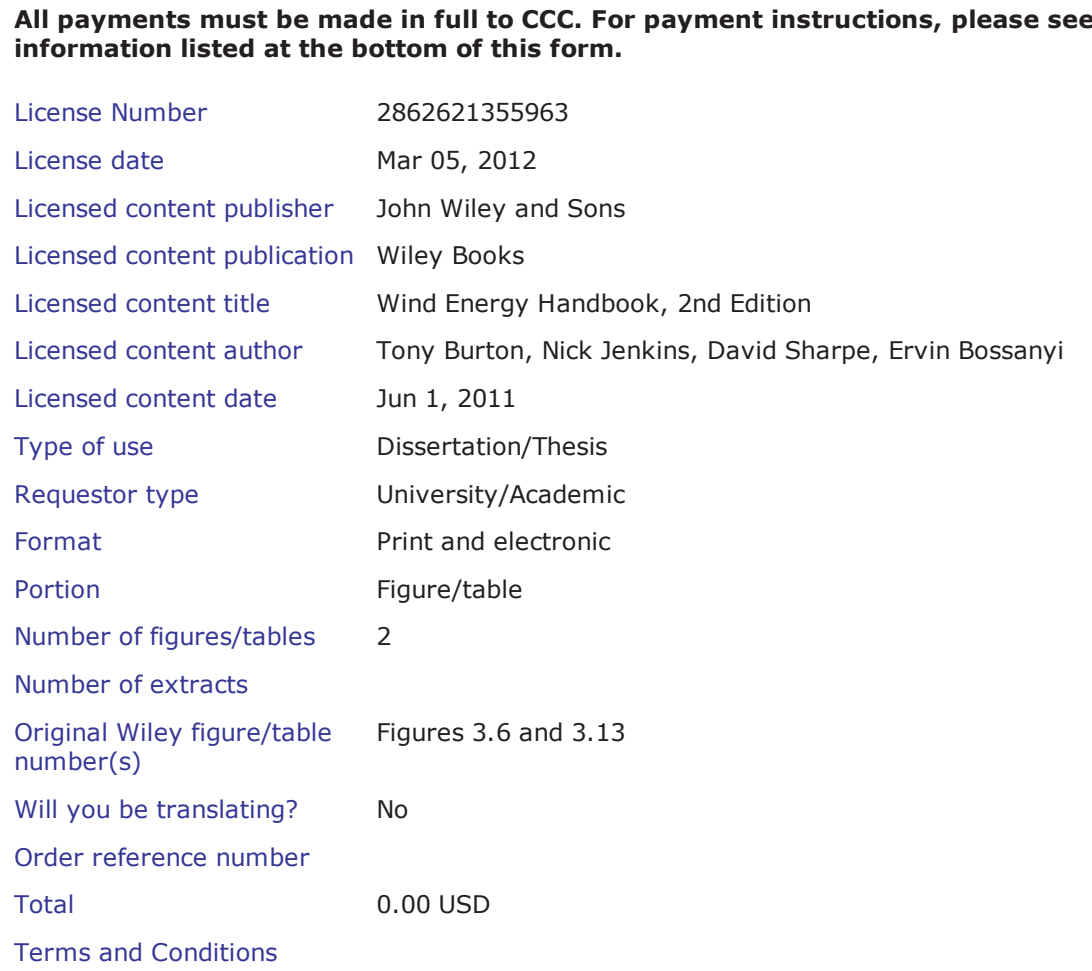

\section{TERMS AND CONDITIONS}

This copyrighted material is owned by or exclusively licensed to John Wiley \& Sons, Inc. or one of its group companies (each a "Wiley Company") or a society for whom a Wiley Company has exclusive publishing rights in relation to a particular journal (collectively WILEY"). By clicking "accept" in connection with completing this licensing transaction, you agree that the following terms and conditions apply to this transaction (along with the billing and payment terms and conditions established by the Copyright Clearance Center Inc., ("CCC's Billing and Payment terms and conditions"), at the time that you opened your Rightslink account (these are available at any time at http://myaccount.copyright.com 